\title{
Der Tapeverband \\ am Sprunggelenk als technischer Bestandteil zur Leistungssteigerung im Sport am Beispiel des Fosbury Flops
}

Eine trainingswissenschaftliche Studie an Hochspringern

\section{DISSERTATION}

zur Erlangung des sozialwissenschaftlichen Doktorgrades der Sozialwissenschaftlichen Fakultät der Georg-August-Universität Göttingen

\author{
vorgelegt \\ von \\ Homayun Gharavi-Nouri \\ aus \\ Teheran / Iran
}

Göttingen 2005 
1.Gutachter:

Prof. Dr. Arnd Krüger

2.Gutachter:

Prof. Dr. Andree Niklas

3.Gutachter:

Prof. Dr. Arturo Hotz

Tag der mündlichen Prüfung:

30.05 .2005 


\section{$\underline{\text { Inhaltsverzeichnis }}$}

1 Einleitung

2 Grundlagen

2.1 Anatomie
2.1.1
Sprunggelenk und Fuß

2.1.1.1 Knöcherner Aufbau

2.1.1.2 Beweglichkeit - Die Neutralnullmethode

2.1.1.3 Das Sprunggelenk

2.1.1.4 Die Architektur des Fußes

2.1.1.5 Der Fußrücken

2.1.1.6 Die Blutversorgung des Fußes

2.1.1.7 Die Fußsohle

2.1.1.8 Die Logen des Unterschenkels

2.1.1.8.1 Das vordere Kompartiment

2.1.1.8.2 Das laterale Kompartiment

2.1.1.8.3 Das hintere Kompartiment

2.1.2 Biomechanik

2.1.2.1 Statische Biomechanik

2.1.2.2 Dynamische Biomechanik 
2.2. Der Hochsprung

2.2.1. Historischer Überblick

2.2.2. Das Reglement

2.2.3. Der Fosbury

2.2.3.1. Technik

2.2.3.1.1. Der Anlauf

2.2.3.1.2. Die Kurve

2.2.3.1.3. Der Absprung

2.2.3.1.4. Die Flugphase

2.3. Häufige Verletzungen des Fußes bei Hochspringern

2.3.1. Pronationstrauma

2.3.2. Muskuläre Verletzungen

2.3.3. Knöcherne Verletzungen

2.4. Der Tapeverband

2.4.1. Geschichte

2.4.2. Grundprinzipien

2.4.3. Das Sprunggelenkstape

2.4.3.1. Technik

2.4.3.2. Biomechanik 
3. Fragestellung

4 Methodik

4.1 Material und Untersuchungsgut

4.1.1 Das Medilogic Fußdrucksystem

4.1.2 Der POSTUROMED $®$ Schwingboden

4.1.3 Der Therapiekreisel

4.2 Versuchsreihen

4.2.1 Propriozeption

4.2.1.1 Versuch 1: Dominanz/Dextrosität

4.2.1.2 Versuch 2: Temperatur

4.2.1.3 Versuch 3: Gleichgewicht und Stabilität

4.2.2 Leistung

4.2.2.1 Versuch 4: Sprunghöhe

4.2.2.1.1 Einbeiniger Sprung am Hang ( $35^{\circ}$ Steigungswinkel)

4.2.2.1.2 Beidbeiniger Sprung auf der Ebenen

4.2.3 Versuch 5: Beweglichkeit

4.2.4 Versuch 6: Der Hochsprung

4.3 Fragebogen zur Ermittlung der anamnestischen und subjektiven Parameter 


\section{Ergebnisse}

5.1 Propriozeption

5.1 .1

Versuch 1: Dominanz/Dextrosität

5.1 .2

Versuch 2: Temperatur

5.1 .3

Versuch 3: Gleichgewicht und Stabilität

\section{$5.2 \quad$ Leistung}

5.2.1 Versuch 4: Sprunghöhe

5.2.1.1 Beidbeiniger Sprung auf der Ebenen

5.2.1.2 Einbeiniger Sprung am Hang ( $35^{\circ}$ Steigungswinkel)

5.2.2 Versuch 5: Beweglichkeit

5.2.2.1 Nach beidbeinigen Sprüngen auf der Ebenen

5.2.2.2 Nach einbeinigen Sprüngen am Hang (35

Steigungswinkel)

5.2.2.3 Einfluss des Hochsprungs auf die Tapestabilität

5.2.3 Versuch 6: Der Hochsprung

5.3 Fragebogen zur Ermittlung der subjektiven und anamnestischen

Parameter

6 Diskussion

7 Zusammenfassung

8 Anhang

9 Literatur 


\section{Einleitung}

Der Hochsprung stellt die einzige Disziplin dar, bei der, ohne Hilfsmittel, die kinetische Energie (Geschwindigkeit) des Körpers in potentielle Energie (Höhe) umgesetzt werden muss. Im Laufe von über einhundert Jahren seit Einführung des Hochsprungs als Wettkampfdisziplin durch die Kelten, sind verschiedene Techniken erprobt und mehr oder minder erfolgreich eingesetzt worden. Am Ende dieser Technikevolution steht aktuell der Fosbury Flop, der seit 1968 den modernen Hochsprung im Spitzenbereich prägt.

Die zunehmende Vermarktung durch die Medien und die wachsende Popularität des Sports stellen zwei zueinander in Wechselwirkung stehende Größen dar, die die Sensationslust nach neuen Rekorden stetig nach oben treiben. Nicht zuletzt deswegen ist das Streben nach Technikoptimierung oder gar die Entwicklung komplett neuer Techniken (HAY 1973) zur verbesserten Überwindung der Schwerkraft das Hauptziel von Athlet, Trainer und Sponsor.

Jedoch hat selbst das Computerzeitalter mit dem Einsatz moderner elektronischer Animationsprogramme die Technik des Fosbury Flops, weder optimieren, geschweige denn ablösen können.

Sich als erfolgreichste Technik zu bewähren, bedeutet, die Kräfte auf den Bewegungsapparat am effektivsten nutzen zu können. Eine höhere Effektivität bedeutet aber auch, das Prinzip der Energieumwandlung zugrundelegend, mit gleicher Geschwindigkeit [v], größere Höhen [h] zu erreichen und damit insgesamt eine höhere Geschwindigkeit umsetzen zu können. 
Beides resultiert in eine höhere Belastung insbesondere des aktiven wie passiven Halteapparates.

In der Bewegungskette zur Umwandlung der zwei Energieformen stellt das Sprunggelenk des Sprungbeines als Hauptbelastungsfocus das schwächste Glied dar. Im Sinne der Weiterleitung physikalischer Kräfte, ist dessen Stabilität die Voraussetzung für eine bestmögliche Nutzung des Einsatzes des restlichen Bewegungsapparates.

In dieser Arbeit soll der Einsatz des stützenden Tapeverbandes am Sprunggelenk als Mittel zur Technikoptimierung getestet und gewertet werden. 


\title{
2 - Grundlagen
}

\author{
2.1 Anatomie
}

2.1.1 Sprunggelenk und Fuß

\subsubsection{Knöcherner Aufbau}

Der Fuß im anatomischen Sinne reicht nicht wie im bayerischen Sprachgebrauch von den Zehen bis zur Hüfte, sondern lediglich vom oberen Sprunggelenksspalt ab nach distal. Er besteht aus 21 Knochen, wenn man die zwei Sesamoide der Großzehe mitzählt. Der Fuß läßt sich von proximal nach distal in Rückfuß, Mittelfuß und Vorfuß einteilen.

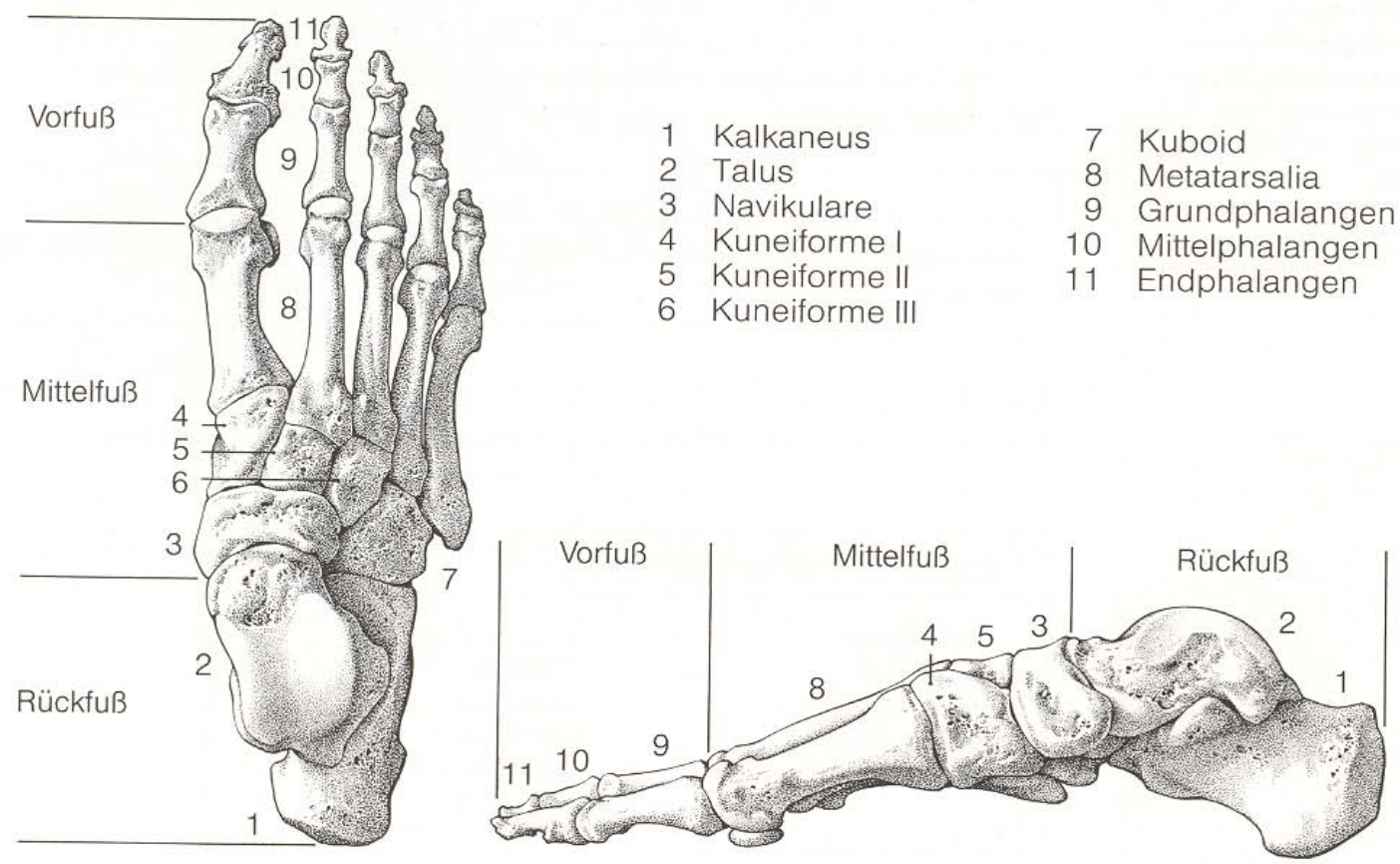

[Abb.2.1.1.1 - Bewegungsausmaße des oberen und unteren Sprunggelenks nach Neutral-Null.

(aus NIETHARD) 
Der knöcherne Rückfuß wird gebildet aus Fersenbein und Sprungbein, genannt Kalkaneus und Talus, welche durch ihre Kommunikationsfläche das untere Sprunggelenk ausmachen. Die Gelenkflächen zwischen Talus, Tibia (Schienbein) und Fibula (Wadenbein) bilden das obere Sprunggelenk. Beide Gelenke werden, wenn es keiner weiteren Differenzierung bedarf, unter dem Begriff „Sprunggelenk“ zusammengefasst.

Der Mittelfuß wird gebildet durch die Tarsal- und Metatarsalknochen (Fußwurzel- und Mittelfußknochen). Die Tarsalia sind in zwei Reihen angeordnet. In der proximalen Reihe befinden sich von medial der Os naviculare und der Os cuboideum. Diese zwei Knochen besitzen nicht nur untereinander eine Gelenkfläche. Das Os naviculare artikuliert nach proximal mit dem Talus und nach distal mit den drei Ossa cuneiforma aus der distalen tarsalen Reihe, welche nach weiter distal mit den Zehenstrahlen I - III in Verbindung stehen. Das Os cuboideum kommuniziert nach proximal mit dem Calcaneus und nach distal direkt mit den Mittelfußknochen der Strahlen IV und V. Dieser Sachverhalt ist besonders wichtig für das biomechanische Verständnis während der dynamischen Belastung, was unter anderem auch im Rahmen des Kapitels über knöcherne Verletzungen deutlich wird.
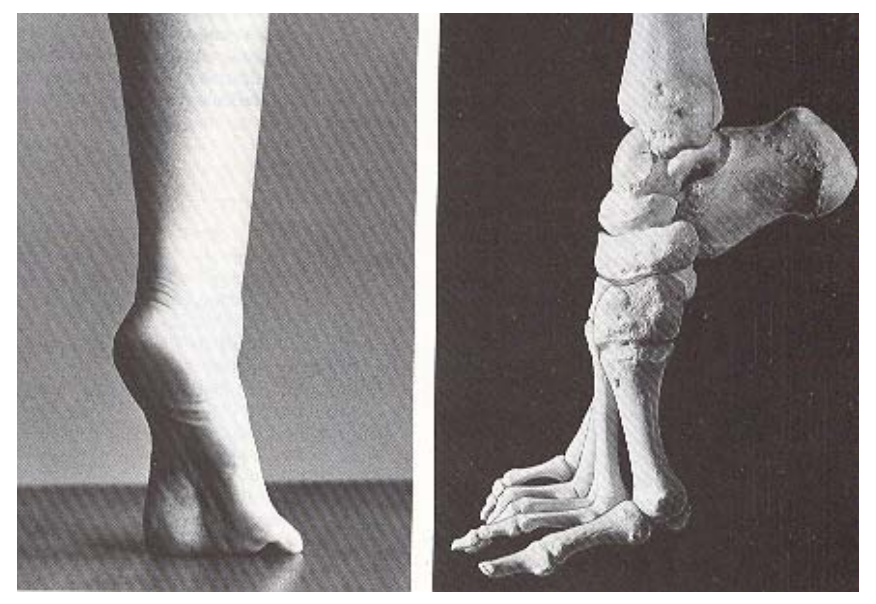

Quelle: BENNINGHOFF 
Die Metatarsalia besitzen jeweils, ähnlich wie die Phalangen im Vorfußbereich auch, am proximalen Ende ihre Basis, am distalen Ende ihren Kopf und dazwischen ihren Körper. Oberflächenanatomisch entspricht die Reihe der Metatarsalköpfchen (MTK) dem Vorfußballen (s. Abb.).

Im Bereich des MTK der Großzehe befinden sich plantar zwei sphärische bis elipsoide Sesambeine, welche der Entlastung des ersten Metatarsalköpfchens, insbesondere während des Ballenganges, dienen. Diese sind wohl ein noch genutztes Relikt aus der phylogenetischen Vierbeinerzeit (s.Abb.).

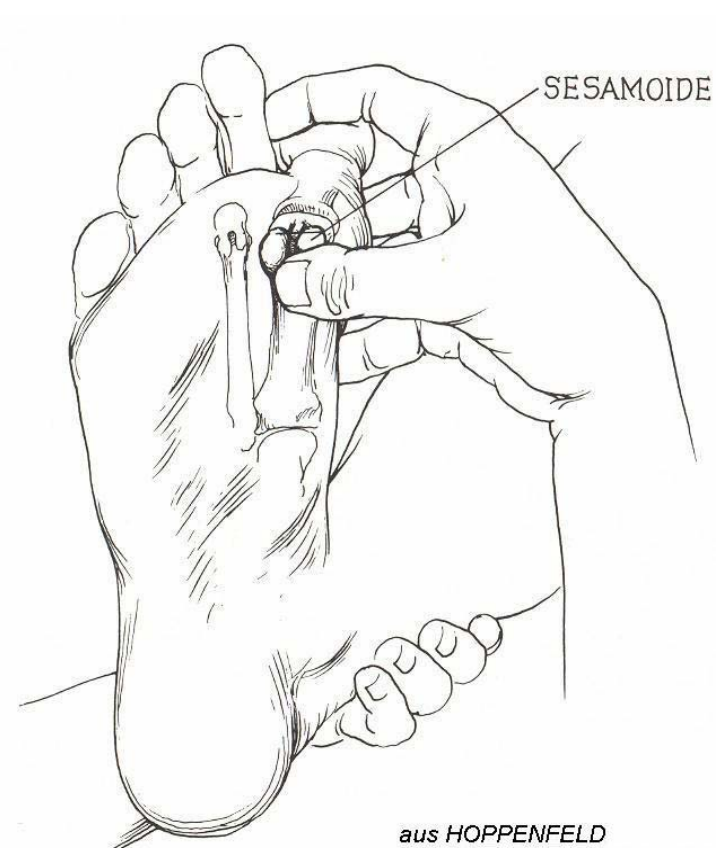

Der Vorfuß besteht aus den

Phalangen. Am ersten Strahl, der Großzehe, werden nur zwei Phalangealknochen unterschieden: ein proximaler und ein distaler Phalanx, welche das Interphalangealgelenk bilden. Die Strahlen II - V besitzen jeweils drei Phalangen: proximal, medial und distal, die untereinander entsprechend je ein proximales Interphalangealgelenk, das so genannte PIP, sowie ein distales Interphalangealgelenk (DIP) bilden. In der Terminologie werden die Gelenke zwischen Mittel- und Vorfuß als Metatarso-Phalangeal (MTP)-Gelenke bezeichnet.

Dieses knöcherne Gefüge wird durch eine Unzahl an derbem, weder flexiblem, noch kontraktilem Bindegewebe, den so genannten Ligamenten, zusammengehalten. In vivo lassen sie sich in den meisten Fällen nicht klar von einander abgrenzen, sodass nur die Faseranteile nach den jeweiligen 
beteiligten Strukturen benannt werden. Das in dieser Arbeit wichtigste Ligament stellt sicherlich das Ligamentum deltoideum, synonym Ligamentum mediale, mit all ihren Anteilen dar.

Die Verletzungsanfälligkeit von Bändern hängt sehr von ihrer Laxizität ab und weist große individuelle Unterschiede auf. Diese Laxizität lässt sich, im Gegensatz zur muskulären Flexibilität, weder auf-, noch abtrainieren. Insofern hängt die im folgenden Kapitel abgehandelte Beweglichkeit nicht nur vom passiven Halteapparat,

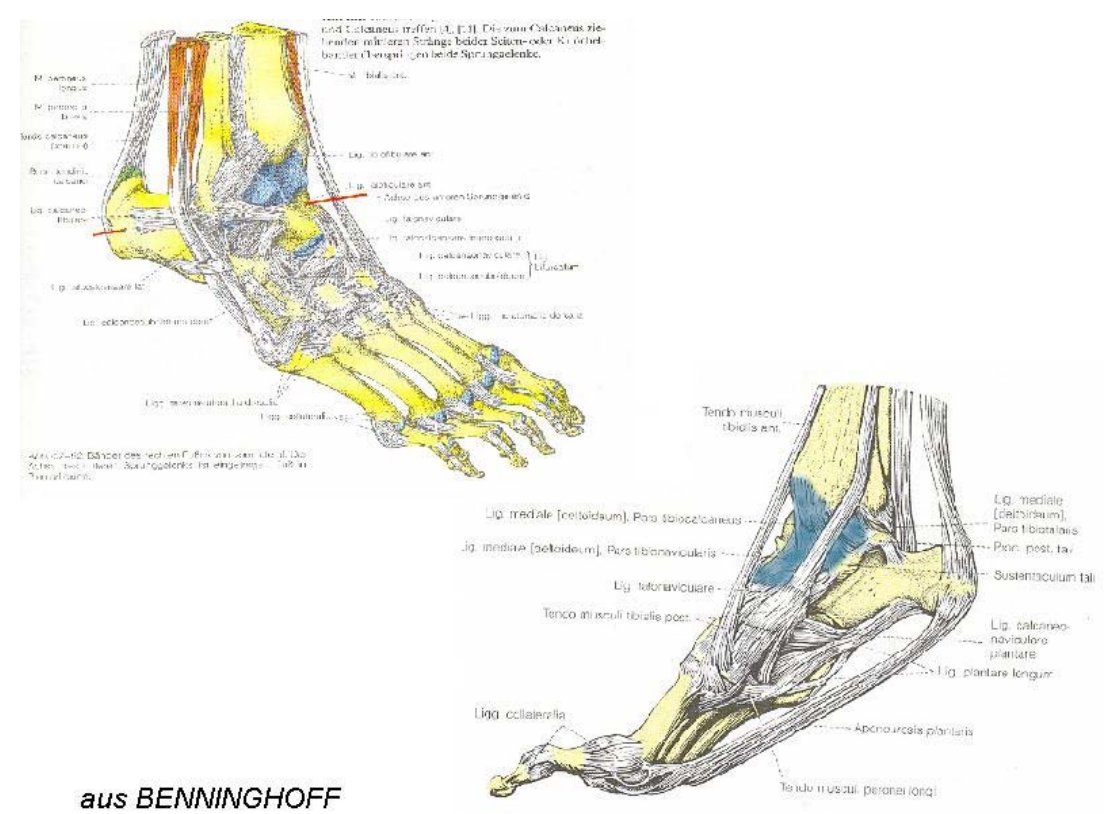
sondern ebenfalls vom konditionellen Zustand des aktiven Systems, der Muskeln, ab. 
2.1.1.2 Beweglichkeit - Die Neutralnullmethode

Die Bestimmung und Beschreibung der Beweglichkeit eines Gelenkes ist nach einem international universellen Standard festgelegt. Man nennt diesen die Bestimmung nach der Neutral-Null Methode. Dabei wird von einer

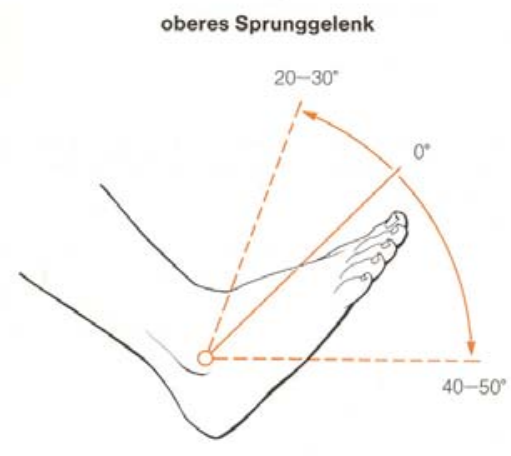

Heben/Senken des Fußes

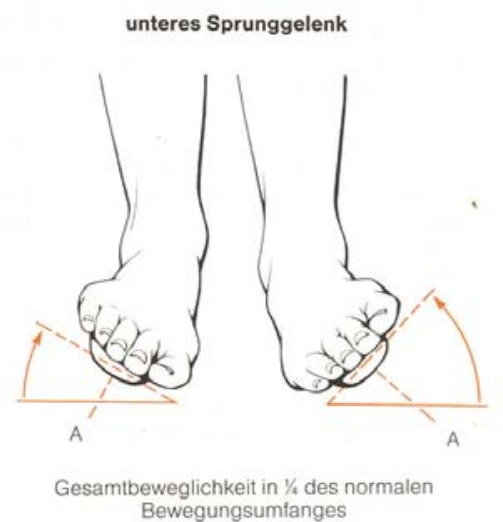

Quelle: Niethart

Neutralstellung des Gelenkes, welche mit 0 Grad definiert wird, das Bewegungsausmaß in den jeweiligen Ebenen in Winkelgrad gemessen. Je nach

Ausschlagsrichtung wird die Gradzahl rechts bzw. links neben die Neutral-Null gesetzt. (s.Abb.)

Am gesunden Sprunggelenk würde es beispielsweise wie folgt lauten (MILLER 1995):

Doescedflection / Extension 30/0/50

Supination/Pronation 50/0/20

Läßt ein Gelenk, seiner Natur entsprechend oder verletzungsbedingt, die Bewegung in eine Richtung nicht zu, z.B. Einschränkung des Ellenbogens in der Extension, wird es Flexion/Extension $160 / 0 / 0^{\circ}$ genannt. Falls die Neutral-NullStellung um $20^{\circ}$ nicht eingenommen werden kann, so würde es so lauten: Flexion/Extension 160/20/0 . 


\subsubsection{Das Sprunggelenk}

Das Sprunggelenk besteht aus zwei Gelenken: das obere und das untere Sprunggelenk. Das obere Sprunggelenk (OSG) wird gebildet durch die distale Tibia und Fibula, die mit mit dem Sprungbein, dem Talus, artikulieren. Aus der

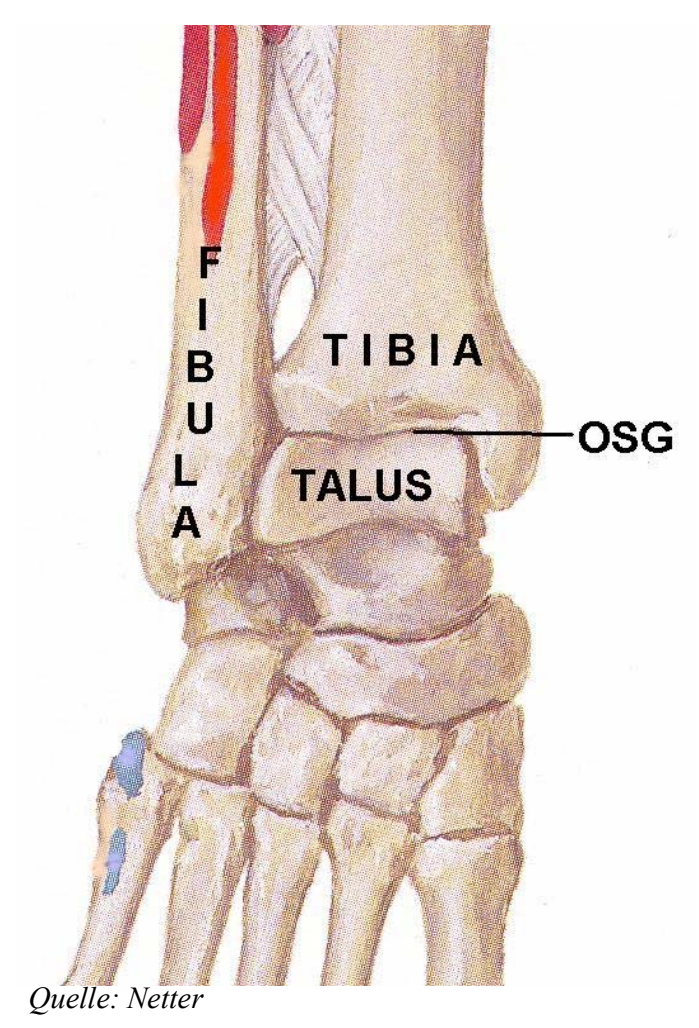
anatomischen Konstellation wird schnell ersichtlich, dass dieses Gelenk lediglich eine Hebung und Senkung, d.h. eine Flexion und Extension des Fußes zulässt und keine seitliche Kippbewegungen im Sinne einer Pronation und Supination erlaubt. (s.Abb.)

Das untere Sprunggelenk (USG) setzt sich aus der distalen Gelenkfläche des Talus und der Gelenkfläche des Fersenbeins, dem Calcaneus zusammen. Die Form dieses Gelenkes läßt im Gegensatz zum oberen Sprunggelenk, anstelle der Flexions-/ Extensionsbewegung, nur die Bewegung in der Pronations-/ Supinationsebene zu.

Die Kombination dieser beiden Gelenke macht das Sprunggelenk zu einem in allen Ebenen beweglichen Gelenk. 


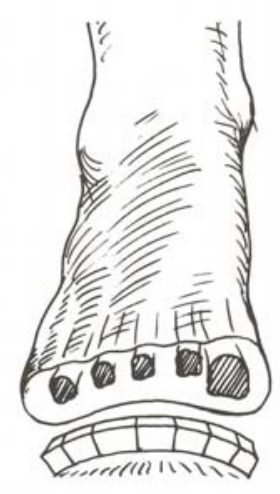

Quelle: Hoppenfeld
Die Anatomie des Fußes gewährleistet durch ein elastisches Quer- und je ein mediales und laterales Längsgewölbe eine optimale Absorption und somit eine möglichst effektive Weiterleitung der dynamischen Druck- und Kraftspitzen während der Fortbewegung.

Dabei ist der mediale Längsbogen stärker ausgeprägt, als der laterale. Der fehlende Bodenkontakt der zentralen Fußsohle selbst im Stand, beugt zudem eine Verletzung der Gefäße, der Nerven, sowie der Muskulatur in dem Bereich vor. Die beschriebenen Bögen werden gewährleistet durch die Form und Anordnung der Fußknochen, dem Verlauf der Bänder und dem der Sehnen extrinsischer Fußmuskeln. Insbesondere die Mittelfußknochen erinnern durch ihre mitunter keilartigen Formen an die verwendeten Steine alter Stadttorbögen oder der bogenförmiger Brücken. Ligamente haben ihren

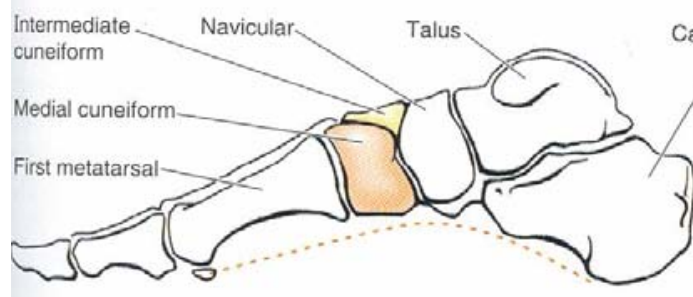

(A) Mediales Längsgewölbe

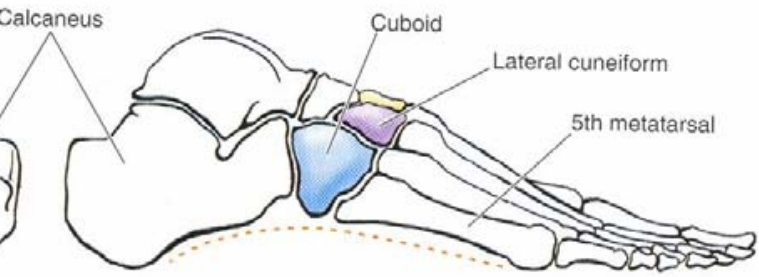

(B) Laterales Längsgewölbe
Quelle: Moore

Ansatz und ihren Ursprung per definitionem jeweils vom Knochen. Hier sind insbesondere die Ligamente plantaris longus und calcaneonaviculare plantare für den Halt des Längsbogens hervorzuheben. Sehnen verbinden stets die 
Muskulatur mit dem Knochen. Sie erlauben dem Muskel die Kraftübertragung über Gelenke auf Knochen zu lenken, die vom Ursprungsort des agierenden Muskels entfernt liegen. So befinden sich die extrinsischen Fußmuskeln im Bereich der Wade. Ihre Sehnen jedoch setzen im Bereich der Fußsohle an. Eine besondere Stützfunktion für das Quergewölbe im Bereich des Vorfußes erfüllt hier die Sehne des Musculus Fibularis Longus, welcher sich quer unter die Fußsohle spannt.

\subsubsection{Der Fußrücken}

Der Fußrücken wird von den Sehnen des M. Extensor Digitorum Longus und des M. Extensor Hallucis Longus überspannt um an den Zehen anzusetzen. Jene Muskeln zählen ebenfalls zu den extrinsischen Fußmuskeln und nehmen ihren Ursprung aus der vorderen Muskellogedes Unterschenkels. Unterhalb dieser Sehnen gelegen, also in tieferer Schicht, findet man die zwei kurzen Fußmuskeln: M. Digitorum Brevis und M. Extensor Hallucis Brevis.

\subsubsection{Die Blutversorgung des Fußes}

Über dem Fußrücken tastbar ist der Puls der Arteria Dorsalis Pedis, die eine Verlängerung der Arteria Tibialis Anterioris (aus der vorderen Loge) ist. Zusammen mit der Arteria Tibialis Posterioris stellt sie die Hauptblutversorgung des Fußes. 


\subsubsection{Die Fußsohle}

Im Gegensatz zur extrinsischen Muskulatur ist die intrinsische Muskulatur des Fußes mit ihren Muskelbäuchen im Bereich des Fußes gelegen. Die intrinsische Muskulatur der Fußsohle neigt in der Schuh tragenden Gesellschaft zur Atrophie und Schwäche, da sie durch das Tragen von Schuhen der stimulierenden Reflexkette des Barfussgehens entzogen bleibt. Das Arrangement der Fußsohlenmuskulatur ähnelt dem der Hand: Eine Gruppe setzt an der Großzehe an, eine Gruppe an der Kleinzehe und eine zentrale Gruppe assistiert den langen Beugern der extrinsischen Muskulatur. Sie werden oft in vier Schichten unterteilt (s. Tab. ).

\begin{tabular}{|c|c|c|c|}
\hline & Muskel & Innervation & Funktion \\
\hline \multirow[t]{3}{*}{$\begin{array}{l}\text { Erste } \\
\text { Schicht }\end{array}$} & Abduktor hallucis & \multirow{2}{*}{ N. plantaris medialis } & $\begin{array}{l}\text { Abduktion und Flexion } \\
\text { der Großzehe }\end{array}$ \\
\hline & Flexor digitorum brevis & & $\begin{array}{l}\text { Flexion der Zehen II } \\
-\mathrm{V}\end{array}$ \\
\hline & Abduktor digiti minimi & \multirow[b]{2}{*}{ N. plantaris lateralis } & Abduktion der Kleinzehe \\
\hline \multirow[t]{3}{*}{$\begin{array}{l}\text { Zweite } \\
\text { Schicht }\end{array}$} & Quadratus plantae & & $\begin{array}{l}\text { Reguliert den Zug des } \\
\text { Flexor digitorum longus, } \\
\text { beugt Zehen }\end{array}$ \\
\hline & \multirow[t]{2}{*}{ Lumbricales } & $\begin{array}{l}\text { N. plantaris medialis für } \\
\text { die Großzehe }\end{array}$ & $\begin{array}{l}\text { Flexion der } \\
\text { Metatarsophalangealgele }\end{array}$ \\
\hline & & $\begin{array}{l}\text { N. plantaris lateralis für } \\
\text { Zehen II - IV }\end{array}$ & $\begin{array}{l}\text { nke. } \\
\text { Extension der } \\
\text { Interphalangealgelenke. }\end{array}$ \\
\hline $\begin{array}{l}\text { Dritte } \\
\text { Schicht }\end{array}$ & Flexor hallucis brevis & N. plantaris medialis & Flexion der Großzehe \\
\hline \multirow{3}{*}{$\begin{array}{l}\text { Vierte } \\
\text { Schicht }\end{array}$} & Adduktor hallucis & \multirow{3}{*}{ N. plantaris lateralis } & Adduktion der Großzehe \\
\hline & Flexor digiti minimi brevis & & काति \\
\hline & Interossei & & $\begin{array}{l}\text { Abduktion und } \\
\text { Adduktion der Zehen }\end{array}$ \\
\hline
\end{tabular}


2.1.1.8 Die Logen des Unterschenkels

Zum besseren Verständnis der Funktion des Sprunggelenkes, sowie der Pathophysiologie der sportspezifische Verletzungen, soll nun auch die Anatomie des Unterschenkels umrissen werden.

Der Unterschenkel ist unterteilt in drei Kompartimente oder auch Logen: eine vordere, eine hintere und eine seitliche. Aufgrund ihres muskulären Inhaltes werden sie auch als Muskellogen bezeichnet. Die hintere Muskelloge wiederum ist in sich in eine oberflächige und eine tiefe Loge unterteilt. Die Blutversorgung und Innervation dieser Kompartimente ist recht übersichtlich und einfach. Die Funktion ihrer Muskeln ist jedoch komplex, da diese an sehr vielen unterschiedlichen Gelenken wirken.

\subsection{Das vordere Kompartiment}

Die vordere Loge, auch Streckerloge genannt, befindet sich lateral der Tibia (Schienbein) und beinhaltet die Muskelgruppen, die für die Fußhebung (Dorsiflexion), die Pronation, sowie für die Extension der Zehen zuständig sind. Diese sind: M. Tibialis anterior, M. Extensor hallucis longus, M. Extensor digitorum longus und der M. Fibularis terius (s. Abb.). Sie werden vom Nervus fibularis profundus und von der Arteria tibialis anterior versorgt. 
2.1.1.2. Das laterale Kompartiment

Die laterale Muskelloge ist auch als Peroneusloge bekannt und enthält den $M$. Fibularis longus, sowie den M. Fibularis brevis. Wie der Name schon verrät, ist einer lang und der andere Muskel kurz. Sie nehmen beide ihren Ursprung vom Wadenbein (Fibula), sind für die Hebung der Fußaussenkante verantwortlich und werden vom $\mathrm{N}$.

Fibularis superficialis innerviert. Die

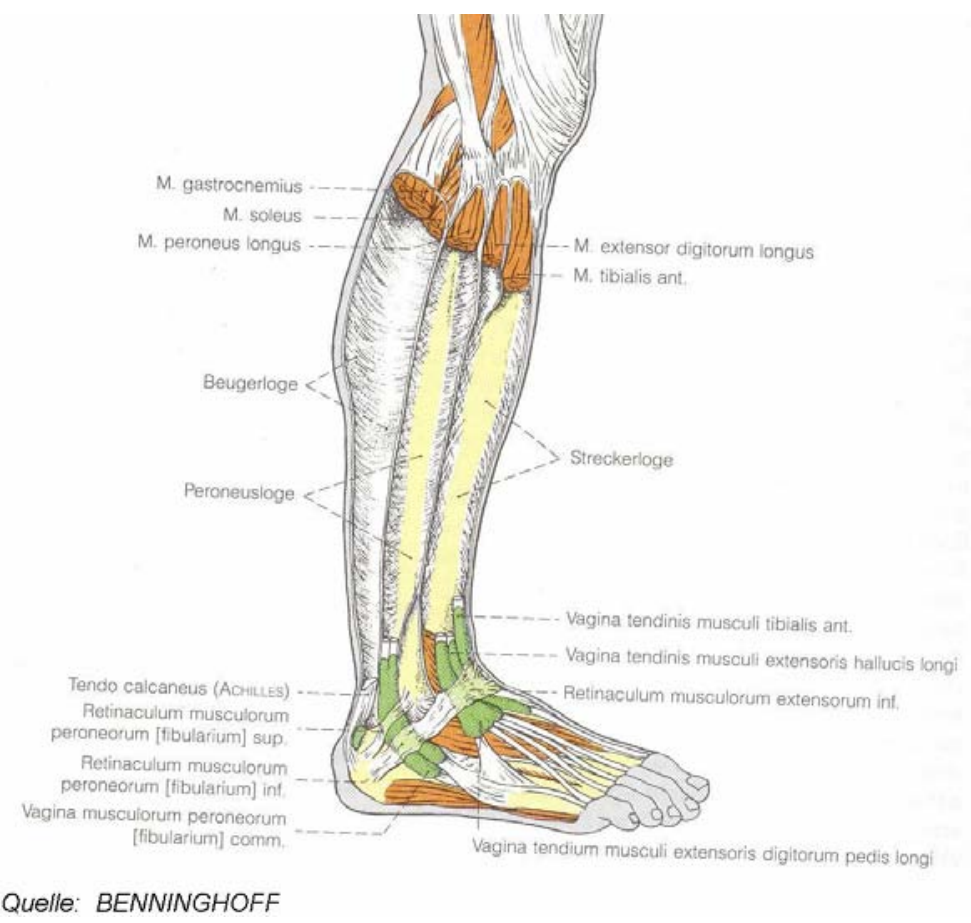
Blutversorgung wird von der Arteria fibularis gestellt, welche sich zwar in der hinteren Loge befindet, jedoch mit perforierenden Ästen die laterale Loge mit versorgt. (s. Abb.) 


\subsection{Das hintere Kompartiment}

Die Muskeln der hinteren Loge prägen die Bezeichnung Beugerloge und sind in zwei Gruppen, einer oberflächigen und einer tiefen Schicht, unterteilt. Der M. Popliteus (tiefe Loge) läuft nur über das Kniegelenk. Der M. Soleus (oberflächige Loge), sowie die tiefen Muskeln Tibialis posterior, Flexor hallucis longus und Flexor digitorum longus laufen nur über das Sprunggelenk (SG). Der doppelbäuchige M. Gastrocnemius und der M. Plantaris (beide superficial) überspannen sowohl das Kniegelenk, als auch das Sprunggelenk. (s.Tab.)

\begin{tabular}{|c|c|c|c|}
\hline Schicht & Muskel & Funktion & Innervation \\
\hline \multirow{3}{*}{ Oberflächig } & Gastrocnemius & Plantarflexion SG, Knieflexion & \multirow{7}{*}{ N. tibialis } \\
\hline & Plantaris & & \\
\hline & Soleus & Plantarflexion & \\
\hline \multirow{4}{*}{ Tief } & Popliteus & Leitet Knieflexion ein & \\
\hline & Tibialis posterior & Plantarflexion SG, suppiniert Fuß & \\
\hline & Flexor hallucis longus & $\begin{array}{l}\text { Flexion Großzehe, Plantarflexion } \\
\text { SG }\end{array}$ & \\
\hline & Flexor digitorum longus & $\begin{array}{l}\text { Flexion Zehen II - V, } \\
\text { Plantarflexion SG }\end{array}$ & \\
\hline
\end{tabular}

[Tab. Muskelgruppen der hinteren Loge] 


\subsubsection{Biomechanik}

\subsubsection{Statische Biomechanik}

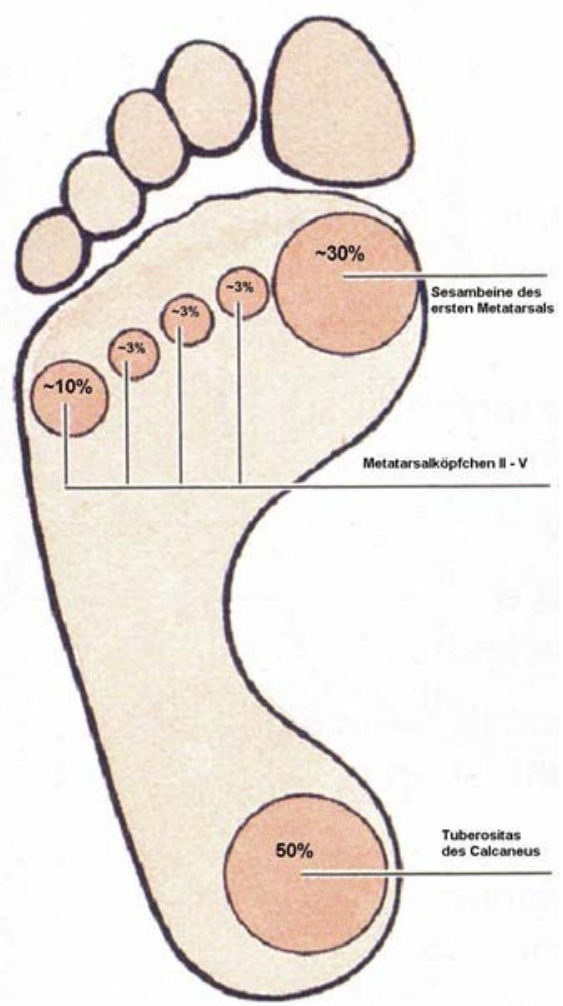

Abb. 2.1.3 Gewichtsverteilung auf die tragenden Punkte des Fußes. MOORE KL 1999

Die statische Belastung des Fußes wird bei gesunden Verhältnissen ungefähr gleichmäßig auf den Calcaneus (Fersenbein) und auf die Metatarsalköpfe verteilt. Untersucht man jedoch die genauen Drücke, so können die folgenden Belastungen in absteigender Reihenfolge beobachtet werden: Calcaneus, Metatarsalkopf I (Großzehenballen), Metatarsalkopf V (Kleinzehenballen) und schließlich die Metatarsalköpfe II - IV. Die Körperlast wird im Verhältnis 1 : 1 auf Ferse und Vorfuß verteilt (s.Abb. 2.1.3). 
Im Vorfußbereich trägt der Großzehenballen 2/3 der Last, wobei die restlichen Zehenballen gemeinsam 1/3 der Vorfußlast übernehmen.

\subsubsection{Dynamische Biomechanik}
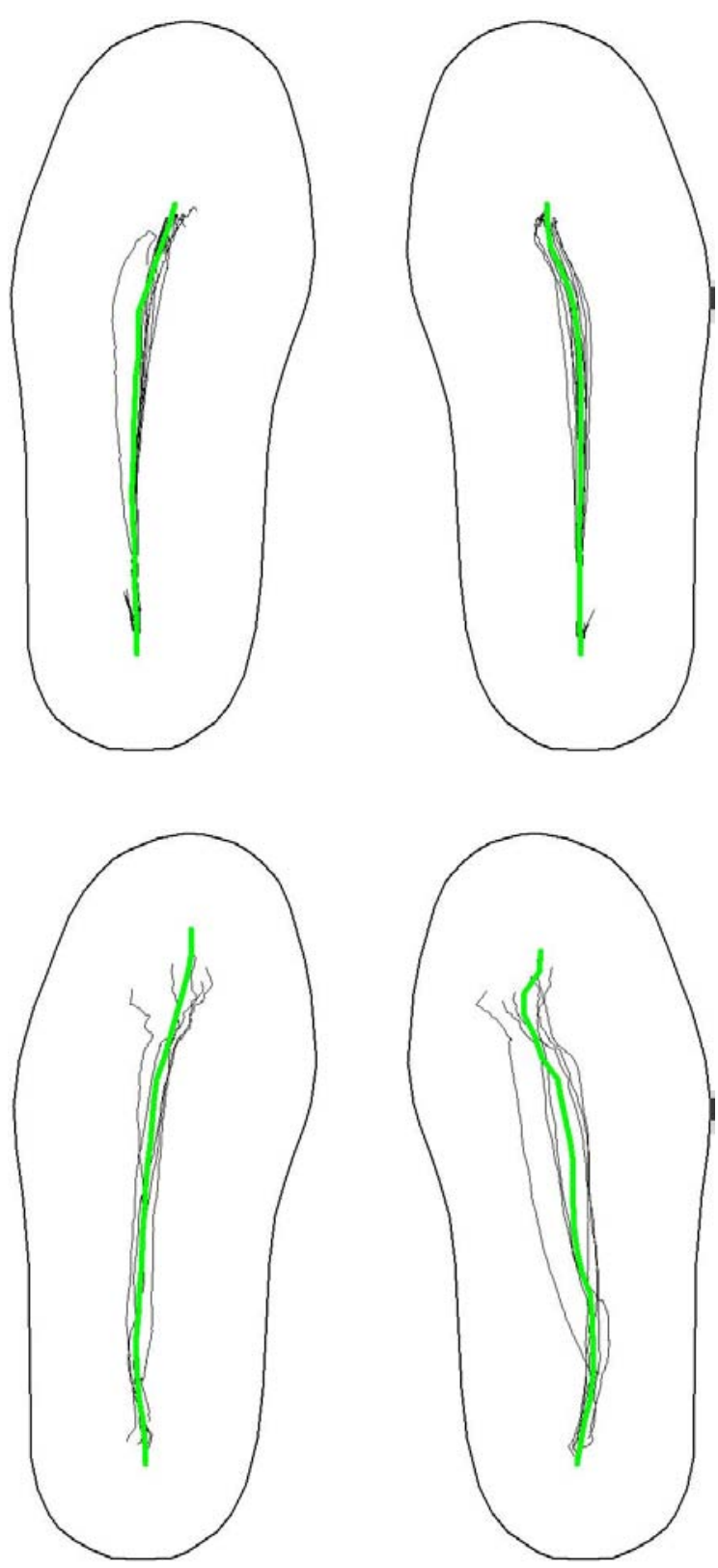

Der Gang läßt sich für jeden Fuß in drei Phasen unterteilen: Absorption, Stand und Abdruck. Bei der Absorption wird das Körpergewicht mit der Ferse aufgefangen und auf den Mittelfuß übergerollt. Dabei wird der Körperschwerpunkt genau über den Fuß gelenkt. Während dieser Standphase wird das andere Bein als Schwungbein nach vorn bewegt, um dort seinerseits mit der Ferse das Körpergewicht aufzufangen und weiter zu leiten. Hierbei wird das Gewicht also vom Standbein über den Vorfuß abgestoßen. Bei gesunden Verhältnissen des Fußes beschreibt der Verlauf des Körperschwerpunktes über der Fußsohle eine mehr oder minder geschwungene Linie von Ferse zum Großzehenballen (s.Abb. 2.1.4).

[Abb. 2.1.4 - Der projizierte Verlauf des KSP über die Sohle beim Gehen einiger Schritte auf ebenem Untergrund. Oben mit Schuhwerk, unten Barfuß. Deutlich zu erkennen: kein Schritt ist einem Zweiten identisch. In Grün: Summe aller KSP-Verläufe.] 
Dadurch, dass der Körperschwerpunkt beim Gang hauptsächlich über den Großzehenballen gelenkt wird, kommt dem medialen Längsbogen die wichtigere Funktion zu. 


\subsection{Der Hochsprung}

\subsubsection{Historischer Überblick}

Im Gegensatz zu den meisten anderen Disziplinen der Leichtathletik, gehörte der Hochsprung nicht zu den olympischen Disziplinen der alten Griechen (Swaddling, 1984). Er wurde von den Kelten erfunden und erstmals um die Jahrhundertwende des 18. und 19. Jahrhunderts bei den schottischen Highland Games ausgetragen. Von dort aus eroberte es den Rest der Welt. Bei den ersten Neuzeitspielen im Jahre 1896 wurde der Hochsprung erstmals international als offizielle Disziplin der Männer ausgeführt. In den USA bestritten Frauen diesen Wettkampf erstmals offiziell auf nationaler Ebene schon im Jahre 1895. Im Jahre 1912 übersprang George Horine als erster Mensch die zwei Meter mit einem „Parallel-Rücken-Roller“. Olympisch wurde die Disziplin erst 1928 in Amsterdam. Bei den Olympischen Spielen 1968 in Mexico City, revolutionierte der U.S. Amerikaner Richard Fosbury die Hochsprungtechnik mit der nach ihm benannten und im modernen Hochsprung bis dato erfolgreichste Technik, und holte die Goldmedallie mit $224 \mathrm{~cm}$. Zwar wurde der „Straddle“ von einigen Autoren, wie ZACHARIAS (1978), WISCHMANN (1987) und BAUERSFELD (1992) als eine dem Fosbury-Flop gleichwertige Technik verteidigt, die Statistik zeigt aber inzwischen, dass die Bestmarken mit dem Straddle ca. $10 \mathrm{~cm}$ unterhalb des Fosbury's liegen (KILLING 1995). Der aktuelle Weltrekord über $245 \mathrm{~cm}$ mit der Fosbury-Technik wird vom Kubaner Javier Sotomayor gehalten. 


\subsubsection{Das Reglement}

Die Grundregeln des Hochsprungs sind seit der zweiten Hälfte des 19. Jahrhunderts im Wesentlichen unverändert. Der Athlet hat drei Versuche jede gewählte Höhe zu überspringen. Als Fehlversuch gelten das Reißen der Latte beim Sprung, ein beidbeiniger Absprung, das Berühren der Landefläche vor dem Absprung, sowie eine Überschreitung des Zeitrahmens nach Aufruf. Der Springer hat ein Zeitfenster von 60 Sekunden nach Aufruf, in dem der Sprung durchgeführt sein muss. Die Ausnahme bildet eine geringe Anzahl von Teilnehmern (zwei bis vier), bei welcher nach Maßgabe der Wettkampfrichter das Zeitfenster auf zwei Minuten erhöht werden kann. Nach jeder absolvierten Runde wird die Latte um mindestens zwei Zentimeter höher gestellt. Nach einem Fehlversuch darf die Latte nicht tiefer gestellt werden. Die Länge, das Gewicht und der Durchmesser der Latte betragen vier Meter, zwei Kilogramm und drei Zentimeter. Die Pfosten, welche die Latte tragen, müssen demnach in einem Abstand von 4.00 - 4.04 Metern zueinander aufgestellt sein. Die Matte, welche die Landefläche darstellt, ist fünf Meter lang und drei Meter breit. 


\subsubsection{Der Fosbury}

\subsubsection{Technik}

Der Hochsprung ist eine technische Disziplin, dessen Erfolg weniger von der Überquerung der Latte, als viel mehr, ähnlich wie bei den anderen Sprungdisziplinen Weitsprung, Dreisprung und Stabhochsprung, vom Anlauf und Absprung geprägt ist. Der Anlauf weist viele Gemeinsamkeiten mit den anderen Sprungdisziplinen auf: Idealerweise beschleunigt der Springer derart, um seine höchste Geschwindigkeit zum Absprung zu erreichen. Ferner muss jeder Springer die Fähigkeit besitzen den Startpunkt seines Anlaufes zentimetergenau abmessen zu können. Beim Fosbury Flop benutzt der Springer jedoch einen $\mathrm{J}$ förmigen Anlauf, der gerade beginnt und in einer Kurve abschließt. Dabei wird das in der Innenkurve liegende Bein zum Sprungbein und die Latte wird rücklings übersprungen.

\subsection{Der Anlauf}

Das Hauptprinzip des Anlaufs besteht darin eine möglichst hohe horizontale Geschwindigkeit (kinetische Energie) aufzubauen. Hierzu beginnt der Anlauf in Form einer geraden Linie direkt auf die Sprunganlage zu. Im letzten Teil läuft der Springer dann in einer scharfen Kurve auf den Absprung zu. Dieser Bogen hat zwei Effekte, die für den Sprung genutzt werden: Einerseits wird ein Rotationsmoment aufgebaut, welcher es dem Athleten ermöglicht die Flugphase mit dem Rücken zur Latte zu vollziehen, um sich bei der Landung um $180^{\circ}$ zum Anlauf gedreht zu haben. Andererseits kann die in der Kurve 
entstandene Zentripetalkraft bei ihrer schnellen Aufhebung aus der Kurvenlage in den vertikalen Absprung zur optimalen Umsetzung der Geschwindigkeit in Höhe (potentielle Energie) umgesetzt werden. Eine saubere Technik vorausgesetzt, können somit höhere Geschwindigkeiten im Anlauf in größere Sprunghöhen umgewandelt werden.

Die folgende Tabelle soll die Korrelation der horizontalen Anlaufgeschwindigkeit mit der vertikalen Abfluggeschwindigkeit, sowie mit der damit letztendlich erreichten Sprunghöhe darlegen (s.Tab.). Diese ist anhand der olympischen Finalisten 1992 ermittelt worden.

\begin{tabular}{|l|c|c|c|c|}
\hline \multicolumn{1}{|c|}{ Name } & $\begin{array}{c}\text { Körpergröße } \\
(\mathbf{c m})\end{array}$ & $\begin{array}{c}\text { Sprunghöhe } \\
(\mathbf{c m})\end{array}$ & $\begin{array}{c}\text { Horizontale } \\
\text { Anlaufgeschwindigkeit } \\
(\mathbf{m} / \mathbf{s})\end{array}$ & $\begin{array}{c}\text { Vertikale } \\
\text { Abfluggeschwindigkeit } \\
(\mathbf{m} / \mathbf{s})\end{array}$ \\
\hline Sotomayor & 194 & 234 & 8,3 & 4,45 \\
\hline Sjöberg & 200 & 234 & 7,4 & 4,05 \\
\hline Conway & 184 & 234 & 7,2 & 4,55 \\
\hline Partyka & 191 & 234 & 7,3 & 4,35 \\
\hline Forsyth & 197 & 234 & 7,1 & 4,15 \\
\hline Sonn & 197 & 231 & 7,5 & 4,25 \\
\hline Henkel & 182 & 202 & 7,2 & 3,70 \\
\hline Astafei & 184 & 200 & 7,1 & 3,75 \\
\hline Quintero & 180 & 197 & 6,6 & 3,70 \\
\hline Kostadinova & 180 & 194 & 7,0 & 3,70 \\
\hline Kirchmann & 181 & 194 & 6,8 & 3,40 \\
\hline Costa & 179 & 194 & 6,6 & 3,60 \\
\hline
\end{tabular}

[Abb. 2.2.3.1 - Geschwindigkeiten des Anlaufes und des Abfluges gemessen bei den Finalisten der olympischen Spiele 1992 (Dapena 1992, Killing 1994a)]

Außerdem kann durch einen schnelleren Anlauf der Absprung weiter von der Latte entfernt vollzogen werden, der Absprungwinkel somit verringert und damit auch die Kraft zur Überwindung der Trägheitskraft verkleinert werden. Insgesamt geht also mit einem schnellerem Anlauf weniger Energie, bei der Umwandlung in Höhe, verloren. Zudem liefert ein zur Latte entfernter 
Absprung dem Springer auch mehr Zeit bis zum Erreichen des Scheitelpunktes der Flugbahn.

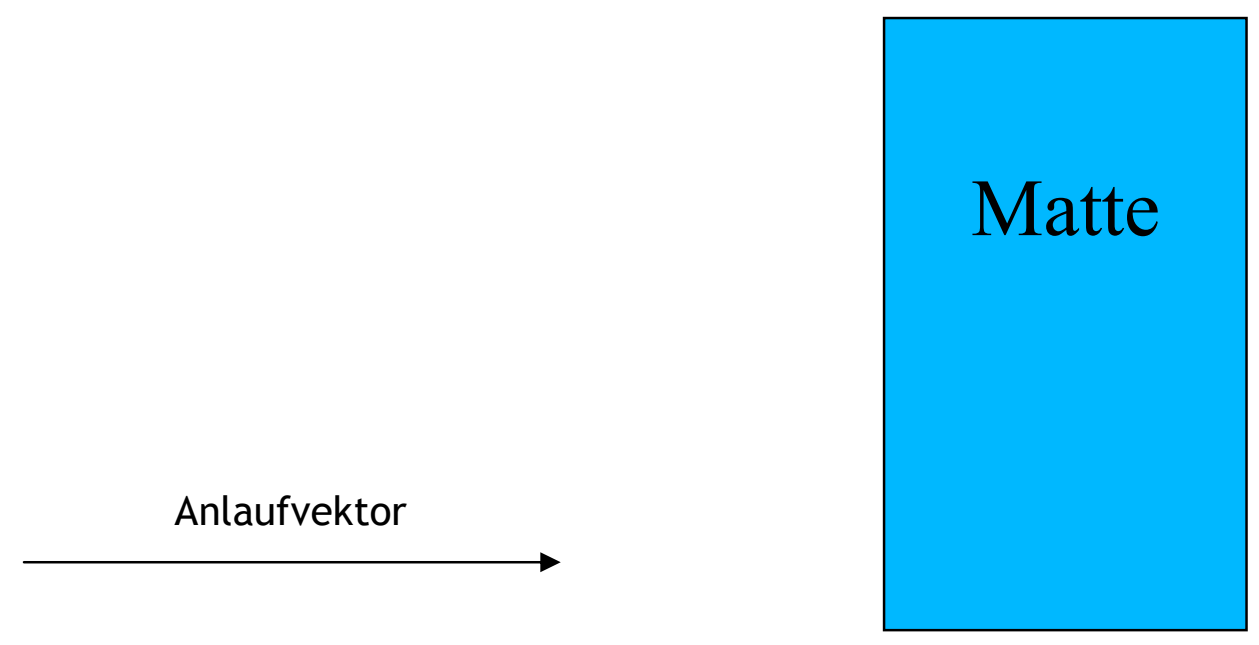

Für KIRST (1978) und BAUERSFELD (1992) ist der Anlauf ein grundlegendes Technikkriterium, das alle anderen Teilbewegungen maßgeblich beeinflussen kann. 


\subsection{Die Kurve}

Radius und Länge der Kurve sind multifaktoriell abhängig individuellen Parametern eines jeden Athleten wie Schrittlänge, Anlaufgeschwindigkeit, Laufrhythmus, Kraft, Körpergröße und -symmetrie, usw. Deshalb nutzt jeder Springer eine eigene individuell zurechtgeschnittene Lauflinie.

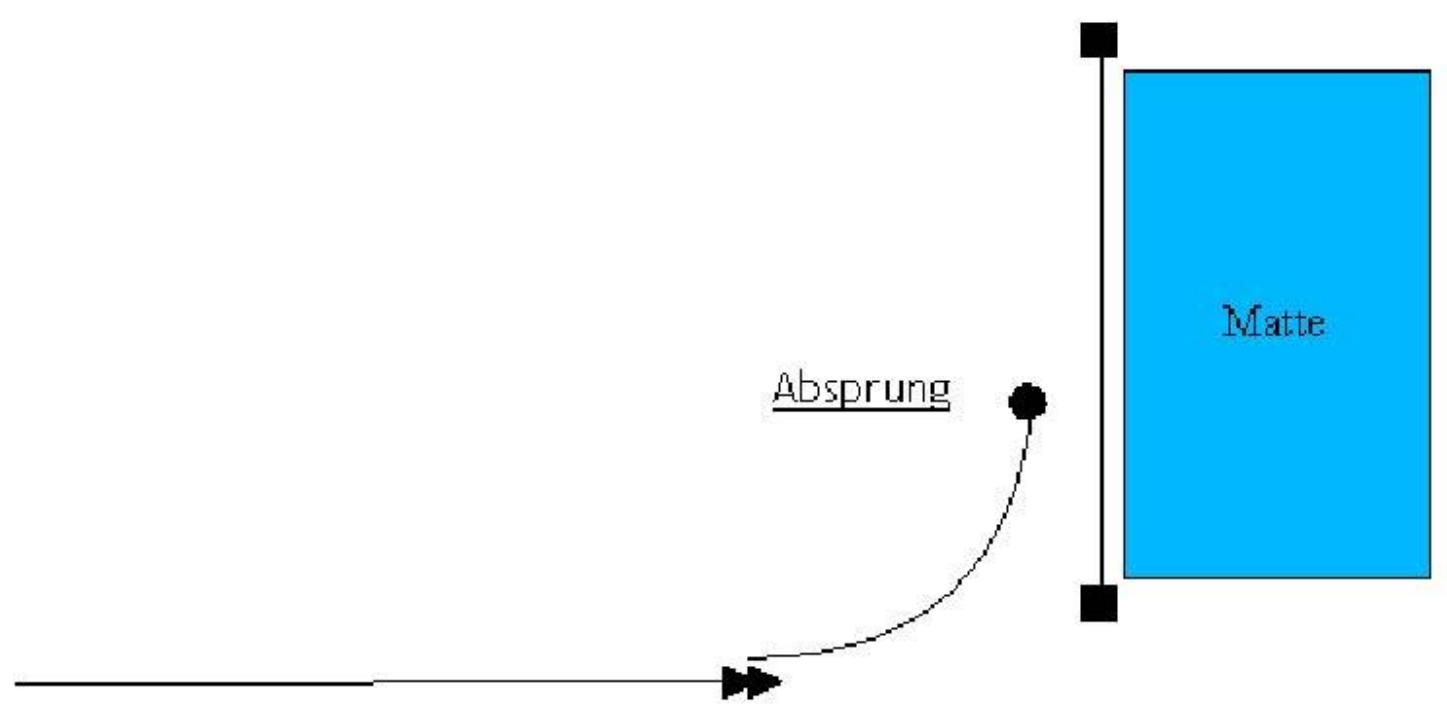


Voraussetzung für inen Körper, sich in einer Kurvenbahn zu bewegen, ist eine angreifende zentripetale (= den Mittelpunkt suchende) Beschleunigung, welche ihn in der Kurve hält. Diese lässt sich anhand der Formel

$$
a_{c}=v^{2} / r
$$

erklären. Es wird deutlich, dass mit zunehmender Geschwindigkeit (v) und/oder kleiner werdendem Radius ( $r$ ), d.h. engere Kurve, eine größere zentripetale Beschleunigung $\left(a_{c}\right)$ notwendig wird. Wenn nun ein Hochspringer mit der Masse (m) auf einer Geraden beschleunigt, erfährt er die Kraft

$$
F=m a .
$$

In der Kurve greift zusätzlich noch eine ins Kurvenzentrum gerichtete zentripetale Kraft an, die sich mit

$$
F_{c}=m a_{c}=m v^{2} / r
$$

errechnen lässt. Hiernach bilden Laufgeschwindigkeit und Kurvenradius die variablen Faktoren, da die Masse des Sportlers ja konstant bleibt. Eine zentripetale Kraft kann verschiedene Ursachen haben: Gravitation (ohne die Anziehungskraft der Erdbeschleunigung würde jeder Körper mit einer Masse tangential zur Erdkrümmung vom Planeten „geschleudert“ werden.), Zugkraft (beim Hammerwurf wird die Kugel über einen Draht befestigt, vom Athleten bis zum Loslassen beschleunigt und in der Kreisbahn gehalten.), Reibungskraft (Beim Hochspringer gewährleistet die hohe Reibungskraft zwischen Spikes und Tartanbelag das Wirken der zentripetalen Kraft. Auf Eis mit glatten Sohlen wäre die Einhaltung einer derartigen Beschleunigung besonders in der Kurve unmöglich.), etc. 


\subsection{Der Absprung}

Der Absprung erfolgt mit dem zur Matte entfernten Bein und stellt im Gesamtgefüge des Hochsprungs die kritischste Phase dar. Genau genommen müssen die letzten zwei Schritte vor dem eigentlichen letzten Bodenkontakt ebenfalls mit zum Absprung gezählt werden.

Drei Schritte vor dem Absprung sollten sich beide Arme in fast vollständig gestreckter Haltung in Brusthöhe vor dem Körper befinden. Der vorletzte Schritt gilt als eine Art Vorbereitung für den Absprung. Dadurch, dass er etwas länger ausfällt, kann zusammen mit dem Absprungschritt eine Art Doppelschrittrhythmus. Mit Senkung der Hüfte wird der Körperschwerpunkt etwas gesenkt um somit eine Vorspannung des Sprungbeines zum Absprung aufzubauen. Die Arme sind dazu inzwischen beide nach hinten unten geschwungen, sodass die Hände sich hinter der Hüfte befinden. Der letzte Schritt wird dann kürzer und schneller vollzogen als die vorausgegangenen Schritte. Der Absprung geschieht mit einem kurzen und schnellen Impuls aller Körperteile und sollte etwa eine volle Armlänge des Athleten von der Latte entfernt liegen. Diese Distanz kann mit der Erfahrung und Geschwindigkeit des anlaufenden Athleten zunehmen. Das Schwungbein sollte maximal nach vorne oben beschleunigt und, sobald der Oberschenkel die Horizontale erreicht hat, plötzlich „blockiert“ werden. Zeitgleich und mit gleicher Dynamik werden die Arme ebenfalls nach vorne oben beschleunigt und in Schulterhöhe blockiert (s.Abb. 2.2.3.1a). Das Schwungbein wird dann rechtzeitig vor der Lattenüberquerung wieder aus der Hüftbeugung in die Streckung gebracht, um die technisch optimale Überstreckung der Hüfte zu ermöglichen. 


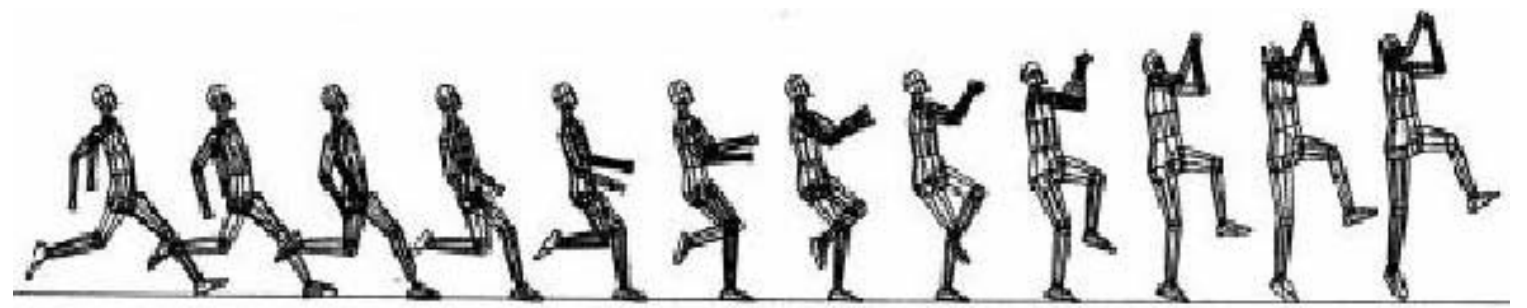

[Abb.2.2.3.1a]

Die Art und Weise, auf welcher der Armschwung geschieht, variiert unter den Athleten sehr. Neben dem beschriebenen parallelen Armvorschwung, sieht man auch oft den Gegenarmschwung, bei dem der näher zur Latte befindliche Arm diagonal zur Gegenseite nach vorne-oben geschwungen wird und somit die axiale Rotation der Körpers unterstützt (s.Abb.).

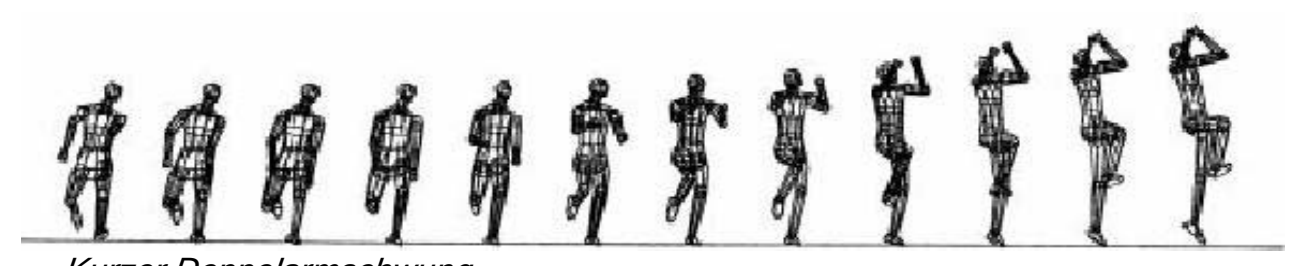

Kurzer Doppelarmschwung

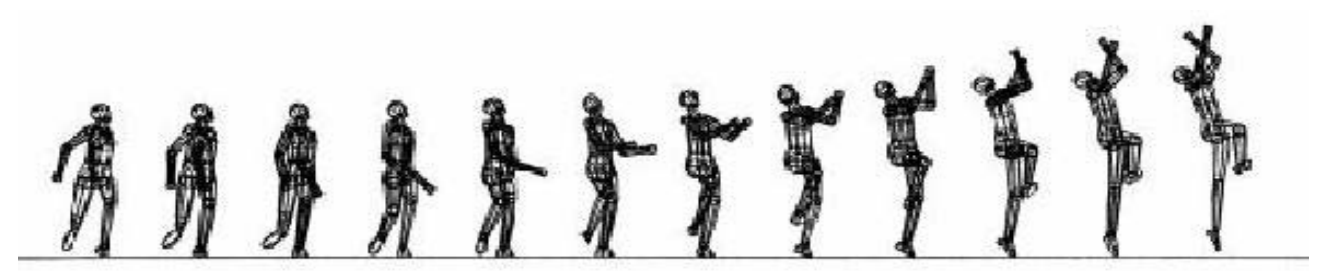

Gegenarmschwung

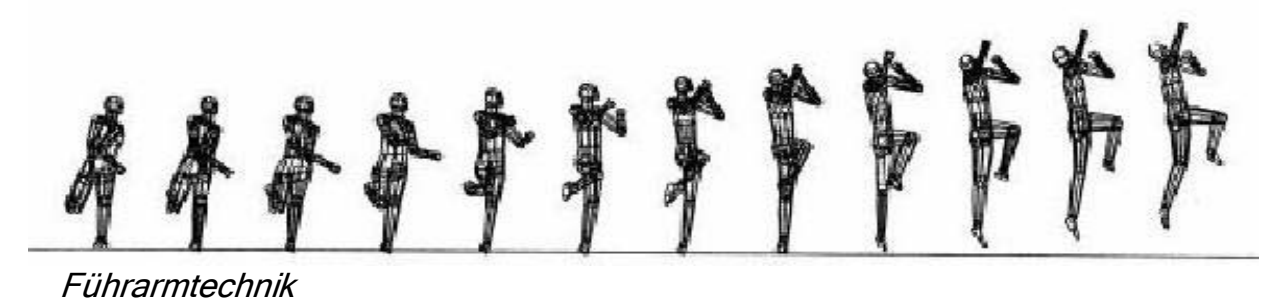

Quelle: DAPENA

Der Effekt des plötzlichen Abbremsens basiert auf dem 3. Newton Gesetz, Actio = Reactio. Physikalisch gilt es hiermit eine möglichst hohe Beschleunigungskraft der Schwerkraft entgegen zu erzeugen. Dies wiederum wird erreicht, indem die 
Beschleunigung der Extremitäten über einen längeren Weg geschieht, als das Abbremsen (negative Beschleunigung).

Das Prinzip dieser Physik läßt sich anhand der Formeln

$$
a=v 2-v 1 / t \text { (a=Beschleunigung, } v=\text { Geschwindigkeit, } t=z e i t)
$$

und

$$
F=m \times a,(F=\text { Kraft }, m=\text { Masse })
$$

erklären (Abb. 2.2.3.1 b).

Zum Start und zum Ende der beschriebenen Bewegung besitzt der Oberschenkel die gleiche Geschwindigkeit (nahezu null). Im Rahmen der positiven Beschleunigung wird der Oberschenkel über einen relativ großen Weg, somit in einem größeren Zeitintervall $(\mathrm{t})$, auf eine maximale Geschwindigkeit gebracht, welche dann im Rahmen der bedeutend schneller (kürzerer Weg = kürzerer Wert für t) ablaufenden Blockierung auf die Ausgangsgeschwindigkeit abgebremst wird. In (1) eingesetzt erhält somit die Größe a während der 
negativen Beschleunigung einen deutlich größeren Absolutwert, als während der positiven Beschleunigung. In (2) lässt sich so bei konstanter Masse (m) der beschleunigten Extremitäten, eine größere Kraft $\mathrm{F}$ für die Blockierungsphase errechnen. Dies geht zugunsten der Überwindung der Schwerkraft.

Voraussetzung für das Wirken der Kräfte ist, dass das Abbremsen der Schwungelemente dann geschieht, während das Sprungbein noch Bodenkontakt besitzt. Die Beschleunigungskräfte von Abstoß und Schwung können sich nur so optimal addieren. Subjektiv sollte der Springer nicht in Richtung Latte, sondern gerade nach oben abspringen. Hierdurch wird aus seiner horizontalen Geschwindigkeit ein vorwärts gerichteter Rotationsmoment gewonnen.

Die in der Kurve wirkenden Kräfte werden beim Absprung in einen lateralen Rotationsmoment verwandelt. Die hieraus resultierende Kraft ermöglicht es dem Springer, Kopf voran, mit dem Rücken zur Latte die gestellte Höhe zu

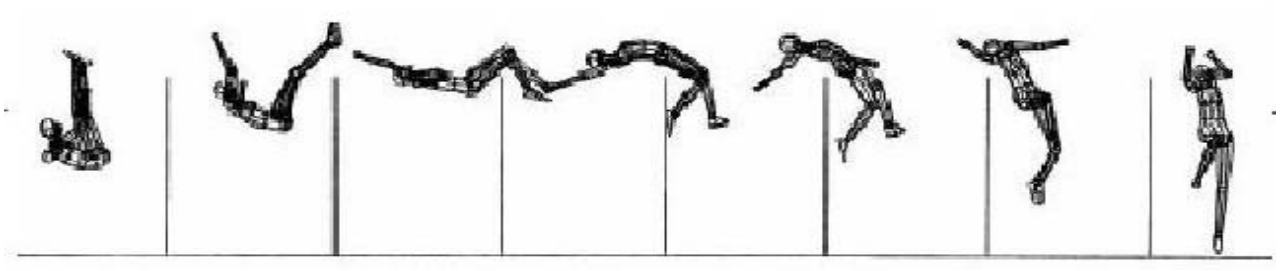

überspringen, um letztendlich auf dem oberen Rücken/Schultern zu landen (s.Abb.). 


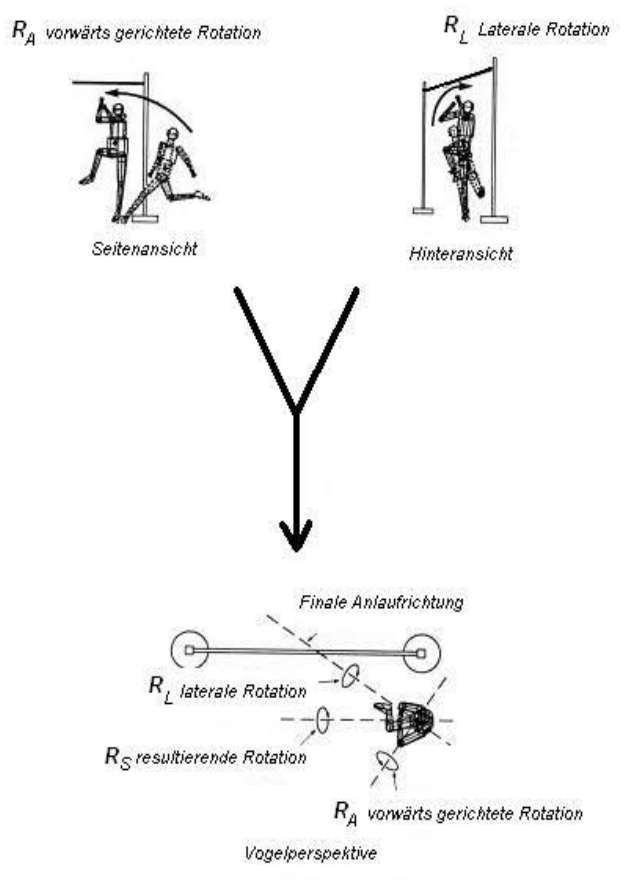

Der letzte und vorletzte Schritt unterscheiden sich von den anderen Schritten im Anlauf dadurch, dass sie nicht mehr mit dem Vorfuß, sondern plan oder sogar mit der Ferse zuerst aufsetzen und über den Fuß abrollen. Hochspringer lassen sich anhand ihres Absprunges grob in zwei Gruppen klassifizieren: Einerseits gibt es die Springer, die den letzten Bodenkontakt sehr kurz und impulsiv gestalten. Dann sieht man häufig auch Springer, so genannte Power Jumper, welche einen kraftvolleren, dafür längeren letzten Bodenkontakt zeigen (BOWERMANN 1991).

Einige Trainer und Athleten argumentieren, dass durch den längeren Bodenkontakt mehr Zeit besteht, nötige Stellungskorrekturen für einen optimalen und kraftvollen Absprungswinkel vorzunehmen. Physikalisch betrachtet, muss die erste Variation jedoch in höheren Sprungklassen, bei 
sauberer Technik, immer dem Power Jump überlegen sein (HUMPHREY 2000), da der letztere Fall mit einer drastischen Reduzierung der Anlaufgeschwindigkeit und einer stärker ausgeprägten Senkung des Körperschwerpunktes vor dem Absprung einhergeht. Das Abbremsen bedeutet ebenfalls eine höhere Belastung, und somit eine höhere Verletzungsgefahr, für die Muskulatur und den Bandapparat der beteiligten Gelenke, wie Knie und Sprunggelenk.

Die wirkenden Kräfte auf das Sprunggelenk des Athleten sind, wie der Sprung im Gesamtablauf, sehr komplex. Aufgrund seiner drei-dimensionalen Ausrichtung ist der während des Absprunges wirkende Kraftvektor mit der genutzten Ausrüstung nicht zu bestimmen. Zur Vereinfachung, muss der Absprung, ähnlich wie beim normalen Gang, in die drei Phasen Absorption, Stand und Abdruck unterteilt werden und jede dieser Phasen getrennt betrachtet werden. Die registrierte Druckkurve des Absprunges zeigt stets ein typisches Muster (s. Abb.).

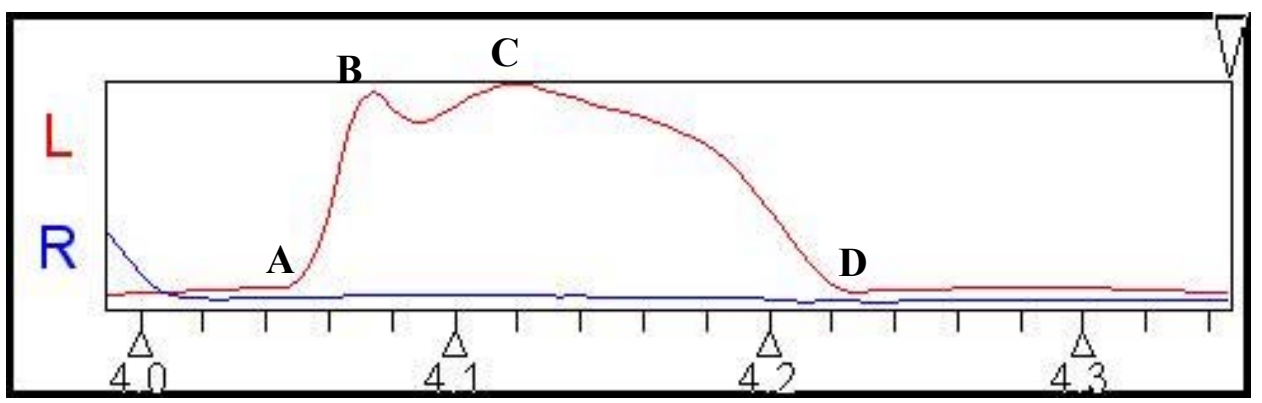

Punkt A beschreibt den ersten Bodenkontakt mit der Ferse während des Absprunges. Die Absorption, als erste Phase des Absprunges, wird durch den Kurvenverlauf zwischen A und B beschrieben. Auffällig dabei ist die große Steigung als Ausdruck eines schnellen Druckanstieges, welcher dann von einem initialen Druckabfall (negative Steigung unmittelbar nach B) abgelöst wird. Dieses Phänomen des kurzzeitigen Druckwegfalles (Dauer: ca. 0,01 - 0,02 sec) ist wahrscheinlich auf die elastische Reaktion aller beteiligten Strukturen 
(Boden, Schuhwerk, Bewegungsapparat) zurückzuführen. Die Standphase endet mit $C$ und geht in die eigentliche Abdruckphase über. Auch diese zeigt einen biphasischen Verlauf mit einer anfänglich positiven Steigung, gefolgt von einem Druckabfall, welche mit dem „Verlassen“ des Bodenkontaktes einhergeht.

\subsection{Die Flugphase}

Während der Flugphase beschreibt der Körperschwerpunkt eine paraboloide Flugkurve. Die Formel zur Errechnung einer Parabel lautet:

$$
f(x)=a x^{2}+b x+c
$$

wobei (a) die Beschleunigung und (b) der Absprungwinkel ist. (c) beschreibt die Höhe des Absprungpunktes. ( c ) bleibt stets konstant, da der Absprung immer vom Boden ausgeht. (a) bezieht sich auf die Beschleunigung, die während des Absprunges generiert wird. Zum besseren Verständnis soll an dieser Stelle daran erinnert werden, dass die Beschleunigung per Definitionem eine Veränderung der Geschwindigkeit darstellt. Die Zunahme der Geschwindigkeit wird als positive Beschleunigung bezeichnet, die Abnahme der Geschwindigkeit als negative, ganz gleich wie hoch oder niedrig die Ausgangsgeschwindigkeit beträgt. Je größer die Anlaufgeschwindigkeit, desto geringer kann die erzeugte positive Beschleunigung durch den Absprung ausfallen. Anders ausgedrückt, bedeutet dies, dass bei geringen Anlaufgeschwindigkeiten eher eine positive Beschleunigung durch den Absprung erzeugt werden kann. Diese Überlegung ist konform mit der mathematischen Rechnung: Hohe Anlaufgeschwindigkeiten erlauben, wenn überhaupt, eine deutlich kleinere positive Beschleunigung als ein langsamer Anlauf. Dies resultiert in eine flache parabole Kurve, was durchaus bei schnellen Springern mit weitem Absprung der Fall ist. Der 
Absprungwinkel (b) wiederum, ist abhängig vom Verhältnis maximal erreichte Flughöhe/horizontale Entfernung des Absprunges von der Latte und stellt die Steigung der Flugkurve dar.

Die Überquerung der Latte

Die bisher beschriebenen Kräfte, welche vom J förmigen Anlauf bis hin zum einbeinigen Absprung mit lattennahem Schwungbein, generiert werden, geben dem Springer Drehmomente um die körpereigene Längs- und Querachse mit. Die Stellung der Arme und Beine in Relation zum Massenschwerpunkt bestimmen das Maß der Trägheit und somit das Maß des Drehmoments. Eine Beschleunigung der Drehung um die Längsachse kann durch körpernahe Führung der Arme erreicht werden. Zieht der Springer die Fersen an, so beschleunigt er die Drehung um seine Querachse. Technisch versierten Springern, die gleichzeitig eine gute Flexibilität mitbringen, gelingt es, ihren Körperschwerpunkt (KSP) unterhalb der Latte her zu führen, während ihr Körper die Latte überspringt. Dennoch haben RITZDORF und CONRAD 1987 ermitteln können, dass selbst die weltbesten Springer den KSP über die Lattenhöhe heben, auch wenn er sie unterhalb passiert.

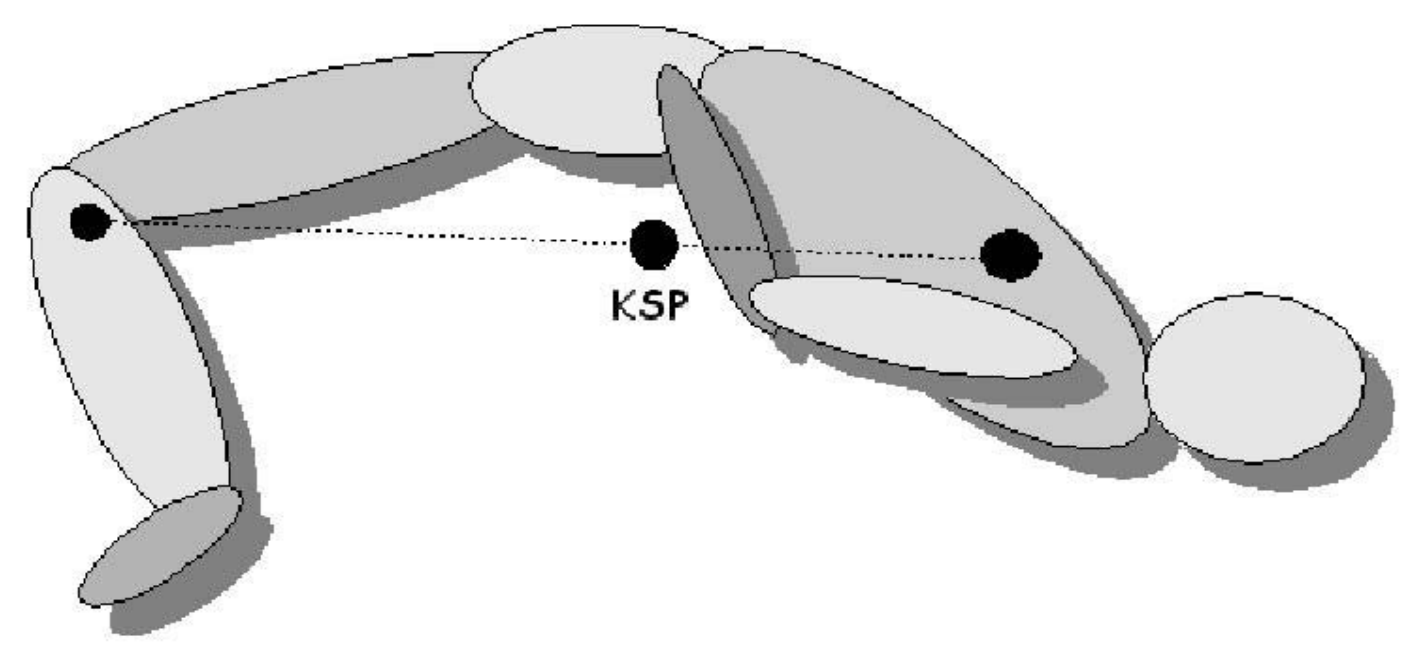

Abb.:Effektive Lage des Körperschwerpunktes (KSP) beim Flop] 


\subsection{Häufige Verletzungen des Fußes bei Hochspringern}

Verletzungen im Leistungssport sind, so akut sie auch oft erscheinen mögen, in den meisten Fällen Resultat einer chronischen Überbelastung. Hochspezifische Trainingsmuster, die in einer speziellen Disziplin zum Erfolg führen sollen, stellen ebenfalls ein hochspezifisches Belastungsprofil mit den entsprechenden Verletzungen dar. So spielen verletzungspräventive Maßnahmen insbesondere im Leistungssport, wo die konditionellen Kapazitäten des Körpers ausgereizt werden, eine wichtige Rolle. Die Vorbeugung von Verletzungen kann in höheren Leistungsklassen mit Leistungssteigerung gleichgesetzt werden, da jede Verletzung eine Auszeit vom Training bedeuten kann. Im Allgemeinen lassen sich fünf Hauptmechanismen aufstellen, die den individuellen Schwellenwert für Verletzungen beim Hochspringer herabsetzen (s.Abb.).

\begin{tabular}{|c|}
\hline Schlecht absorbierender Bodenbelag \\
\hline \hline Überbelastung, Ermüdung \\
\hline Unzureichende physische Vorbereitung \\
\hline Schlechte Technik \\
\hline Anatomische Prädisposition \\
\hline
\end{tabular}

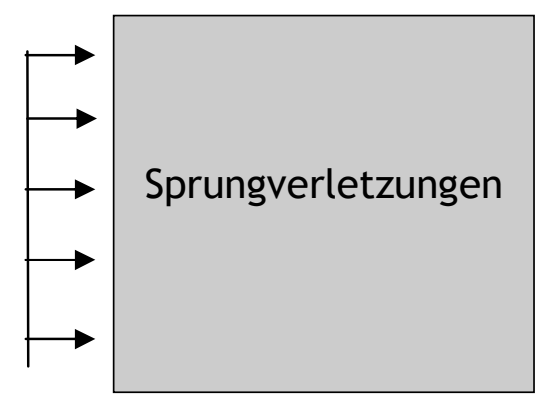

Der elastische Hartgummibelag (Tartan) ist auf Sportanlagen mittlerweile zum Standard geworden. Er ist durch seine Elastizität schockabsorbierend und energiekonservierend (federnd) und zeigt auch nach langem repetetiven Gebrauch keine Unebenheiten in seiner Oberfläche. Durch diese Qualitäten hat er den Beton und Aschenbelag abgelöst. Das im Hochsprung geläufige Schuhwerk (Spikes) ist, ähnlich wie das der Sprinter, ungepolstert. Sicherlich 
lassen sich die Elemente Ausdauer und Kraft auch mit gepolstertem Schuhwerk trainieren. Einzelne sportartspezifische Bewegungselemente, die isoliert als so genannte Drills geübt werden, können in der Regel ebenfalls mit zusätzlich gepolsterten Sohlen trainiert werden. Auf diese Stoßdämpfung sollte, soweit möglich nicht verzichtet werden, da zur Automatisierung der Technik viele Wiederblungen notwendig sind.

Die Faktoren Ermüdung und unzureichende physische Vorbereitung liegen in erster Linie in der Verantwortung des Betreuers. Ein angemessener Trainingsplan mit vorgesehenen Trainingspausen, welcher den Sportler kontrolliert auf einen Wettkampf bzw. auf ein Leistungsziel vorbereitet, ist essentiell für gesunde erfolgreiche Saison. Erholungsphasen gewährleisten die Leistungssteigerung.

Eine saubere Technik nutzt die Anatomie für eine möglichst effektive Arbeit. Die richtige Technik minimiert Fehlbelastungen, nicht aber die Überbelastung.

Anatomische Variationen können durch individuell angepasste Ausrüstung kompensiert werden. Dies ist der Fall, wenn es sich bei der Anatomie um eine so genannte Normvariante handelt. Im Behindertensport muss, entsprechend der neuro-muskulo-skelletären Veränderung, die Technik verändert bzw. modifiziert werden. 


\subsubsection{Pronationstrauma}

Bei der Pronation wird die Fußsohle nach außen und der mediale Knöchel nach innen bewegt.

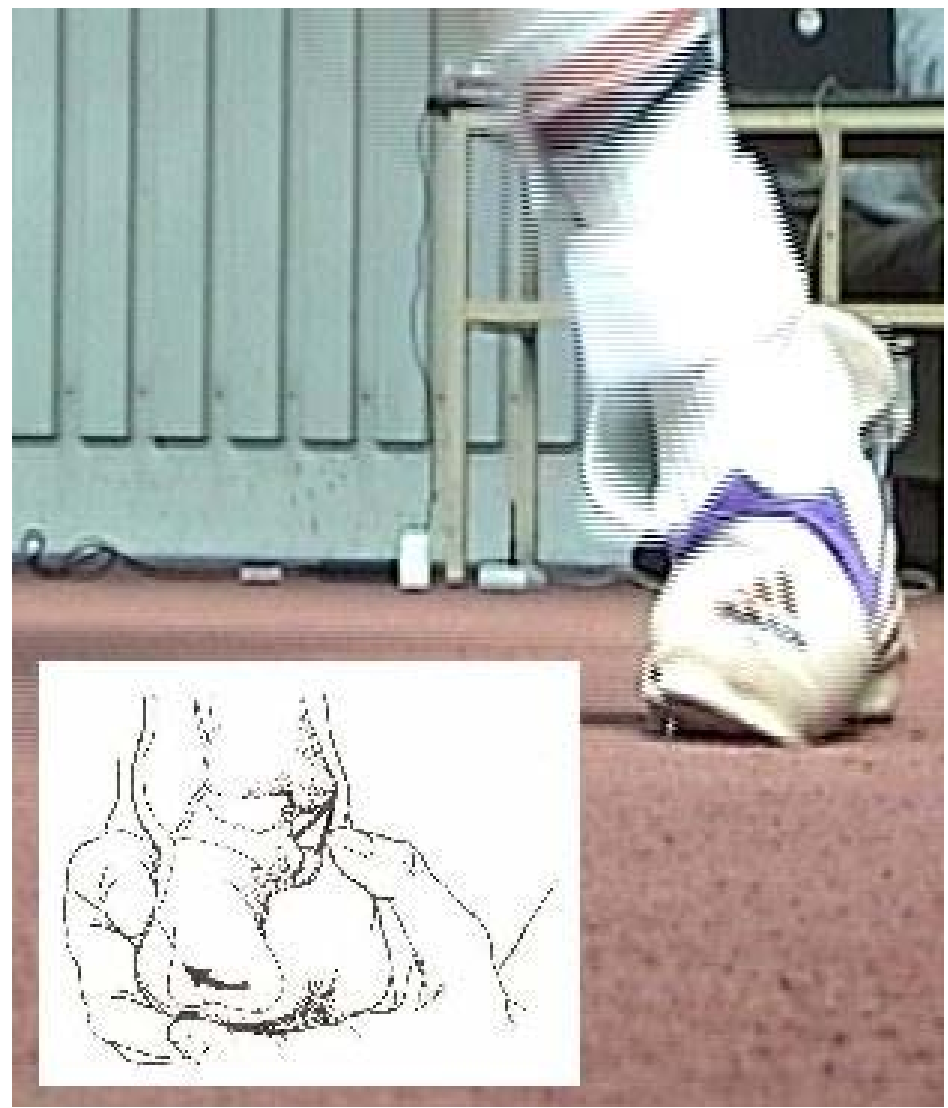

Abb. 2.4.1 - Das Ligamentum deltoideum wird durch die Pronation während der Stemmphase des Absprunges stark strapaziert (Zeichnung aus HOPPENFELD). 
Um zu verstehen, dass es sich beim Pronationstrauma des Hochspringers um einen komplexen Mechanismus handelt, soll an dieser Stelle nochmals an die physikalischen Kräfte während des Absprunges erinnert werden:

(3) $F_{c}=m a c=m v^{2} / r$.

Es wird ersichtlich, dass mit steigender Masse $\mathrm{m}$, sowie mit zunehmender Geschwindigkeit $v$ und bei kleiner werdendem Kurvenradius $r$, die den Fuß in die Pronation zwingende Kraft zunimmt (s. Abb. 2.4.1).

So kommt es, dass das Ligamentum deltoideum einer extrem hohen Belastung ausgesetzt sein kann, welche in eine Verletzung resultieren kann. Angemessenes Schuhwerk und Vermeidung einer extremen Außenrotation und Pronation beim Aufsetzen zum Absprung können das Verletzungsrisiko dieses Ligamentes mindern. 


\subsubsection{Muskuläre Verletzungen}

Wie oben erläutert, ist die Anlaufgeschwindigkeit beim Hochsprung, sowie bei allen anderen Sprungdisziplinen, von essentieller Bedeutung für den Absprung und die darauf folgende Flugphase. Je höher die Anlaufgeschwindigkeit, desto ausgeprägter ist der dem Anlauf entgegengesetzte Stemmschritt zur Umsetzung der Geschwindigkeit in Höhe. Die beim Power Jumper verlängerte Stemmphase erhöht das Risiko von Muskelverletzungen insbesondere im Bereich der Gastrocnemii, sowie des Quadriceps femoris und der Kniebeuger. Bei guten Springern mit kurzem Stemmschritt sind diese Verletzungen seltener anzutreffen (WENTZ 1997).

\subsubsection{Knöcherne und ligamentäre Verletzungen}

GRAFF et. al. (1986) beobachteten bei Hochspringerinnen gehäuft Stressfrakturen des Os Naviculare pedis und versuchten dies mit den verschiedenen Fußformen zu korrelieren. Die Stemmphase verläuft über die Ferse und der Abstoß über den Großzehenballen. Bei unseren Versuchen haben wir beobachten können, dass es während des Absprunges zwei Druckspitzen über der Fußsohle gibt: Die erste entwickelt sich im Bereich der lateralen Ferse und geht mit der Stemmphase einher. Die zweite baut sich während der Abstoßphase unter den Metatarsalköpfchen (Zehenballen) I - III auf (s.Abb.). 


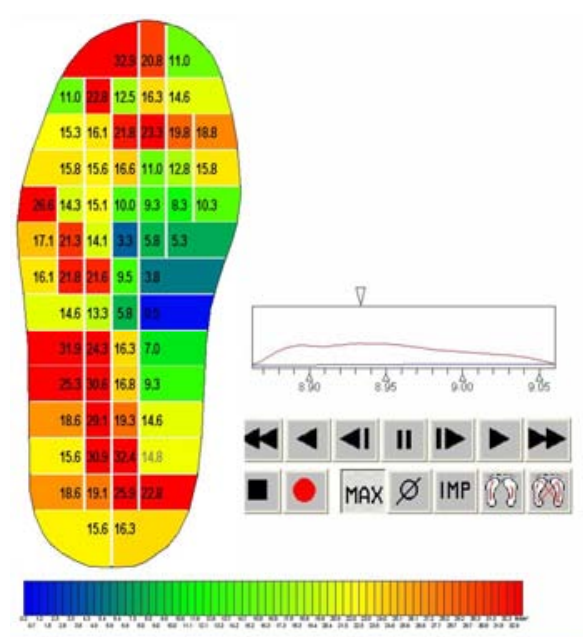

Abb. 2.3.3a-Typische Verteilung der Maximaldrücke während aller drei Phasen des Absprunges. Auffällig sind die Druckspritzen im Bereich der Ferse und der Zehenballen I - III.

Der Os naviculare nimmt im Rahmen dieser dynamischen Druckverteilung, aufgrund seiner Lage im anatomischen Gefüge des Fußes, eine Sonderstellung ein. Die während der Stemmphase beim Absprung eingegangene extreme Pronationsstellung des Fußes, wird über die vorderen Anteile des Ligamentum deltoideum stabilisiert. Somit wird der Os naviculare dabei einer sehr hohen Zugbelastung ausgesetzt (s.Abb.2.3.3b).

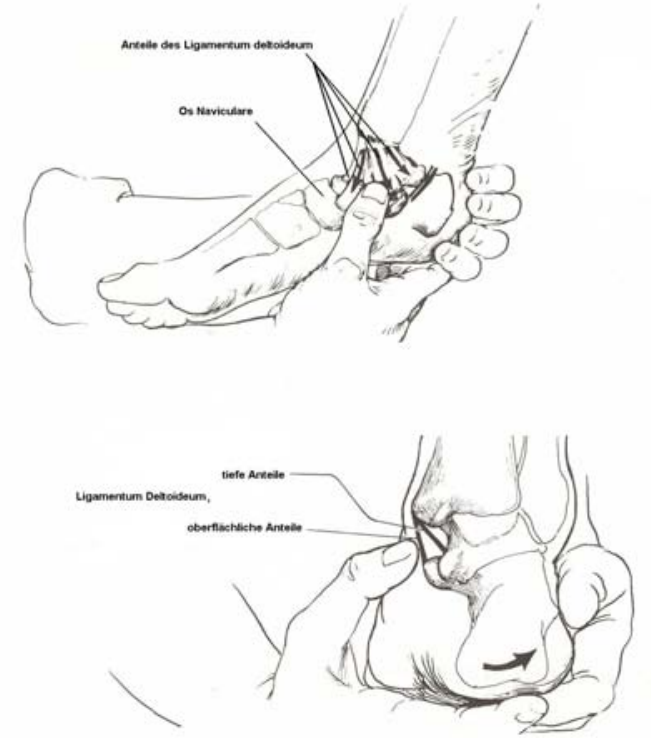

Abb. 2.4.3. $b$ - Das Ligamentum deltoideum. Vom medialen Malleolus zieht es zu Calcaneus, Talus und zum Os naviculare und stabilisiert den medialen Sprunggelenksspalt während der Pronationsbewegung (aus HOPPENFELD). 
Der Abstoß geschieht hauptsächlich über die medialen Zehenballen. Die Translation der Kraft verläuft hierbei den Os naviculare und den Talus (s.Abb. 2.4.3c).
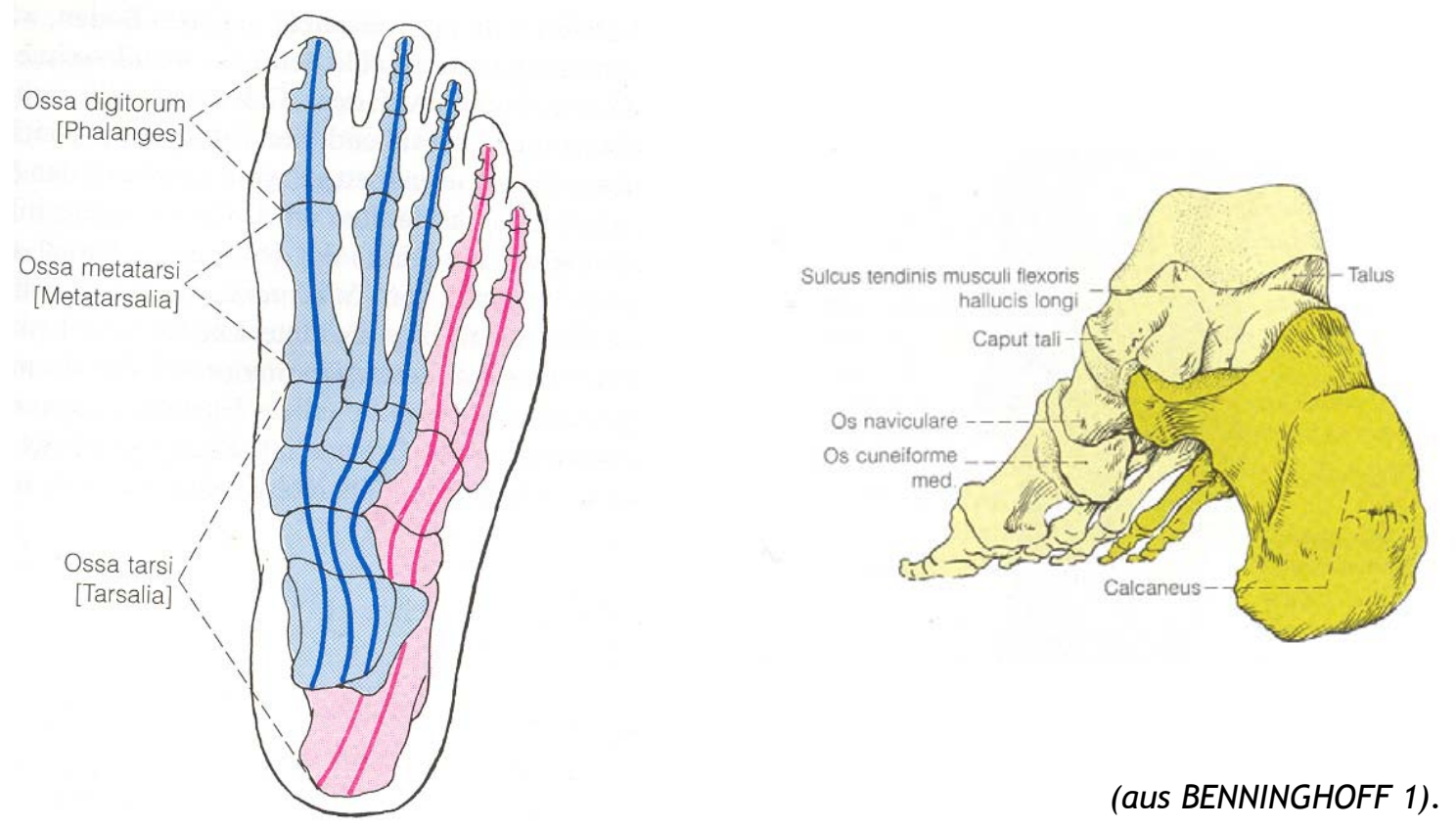

Abb. 2.4.3c - Kräfte über die Strahlen I - III werden über den Os naviculare und Talus geleitet

Die beschriebenen Sachverhalte zugrundelegend, läßt sich folgern, dass der Os naviculare während der höchsten Krafteinwirkung, nämlich der Stemmphase, einer Zugspannung ausgesetzt ist und während des Abstoßes einer Druckbelastung ausgesetzt ist. 


\subsection{Der Tapeverband}

\subsubsection{Geschichte}

Das Tape als funktioneller Stützverband wurde nicht nur zur Stabilisierung des Bewegungsapparates verwendet. Älteste Überlieferungen reichen über 3000 Jahre zurück. Sie beschreiben harzgetränkte Leinwandstreifen, welche, ähnlich wie die heutzutage in der Wundversorgung verwendeten „Steri-Strips“, zum Annähern und Stabilisieren von Wundrändern eingesetzt wurden. Die Idee der Verletzungsprophylaxe durch Verbände kommt erstmals bei den Gladiatoren der Römerzeit vor (MONTAG 1993). ANDRY beschreibt 1744 Leinentücher und elastische wie unelastische Binden, welche er zur Behandlung von Distorsionsverletzungen („Umknickverletzungen“) einsetzt. Paul Beiersdorf entwickelte 1892 dieses Prinzip zu einem Produkt, das heute als Tape gebräuchlich ist. Er beschichtete unelastische Zellwolle einseitig mit ZinoxidKautschuk-Harz-Kleber und bot es als Pflasterrolle an. 


\subsubsection{Grundprinzipien}

Ein Tapeverband wird in der Regel nur für die Dauer einer Trainingseinheit oder eines Wettkampfes eingesetzt. Im Falle einer Stabilisierung nach Verletzungen stellt das Tape eine Unterstützung, aber keinen Ersatz zum laufenden Rehabilitationsprogramm des verletzten Athleten dar. Tape kann zur Erreichung der folgenden Zielen eingesetzt werden:

- Einschränkung einer bermäßigen Beweglichkeit eines betroffenen Gelenkes.

- Kompression der Weichteile zur Verhinderung von Schwellung.

- Stabilisierung der involvierten anatomischen Strukturen.

- Fixierung eines primären Wundverbandes.

- Schienung einer betroffenen Extremität oder ihrer Abschnitte.

- Fixierung einer Schiene.

Tapeverbände, die mindestens ein Gelenk überspannen und somit stabilisieren sollen, werden grundsätzlich nach demselben Prinzip angelegt: Zunächst werden proximal und distal des Gelenkes semizirkuläre „Anker“ gelegt, welche den nachfolgenden Zügeln als Anhaftpunkt dienen. Sämtliche Zügel und Figuren wiederum dienen den auf sie folgenden Zügel und Figuren erneut als Anker. Einen Standard, nach welchem ein bestimmtes Gelenk stabilisiert bzw. eine bestimmte Verletzung vorgebeugt werden soll, gibt es nicht. Man trifft auf ein Unzahl an Variationen, die letztendlich jedoch den selben Sinn und Zweck erfüllen sollen. Nach Vollendung aller funktionell benötigten Zügel, sollten nicht gedeckte Areale, man bezeichnet diese auch als Fenster, mittels semizirkulärer Streifen überklebt werden. Im Fachjargon als „Fill-ins“ bekannt, werden diese immer von proximal nach distal angelegt, um dem Sportler das 
Überstreifen der Socken ohne Aufrollen der Tapestreifen zu ermöglichen.

Bei Wunden, Frakturen und Durchblutungsstörungen, beispielsweise bei den Grunderkrankungen Diabethes (Zuckererkrankung), Varikosis (Krampfadern), arterielle Verschlusskrankheit, usw., ist ein Tapeverband nicht indiziert. 


\subsubsection{Das Sprunggelenkstape}

Beim Sprunggelenkstape wird in der Regel der Unterschenkel von distal aufwärts bis zur Hälfte rasiert. Athleten und Athletinnen, die auf die Rasur verzichten möchten, tun dies gewöhnlich nur einmal. Eine gründliche Rasur vereinfacht das Entfernen des Verbandes sehr. Der Athlet nimmt auf einem Behandlungstisch platz, sodass sein halber Unterschenkel über die Tischkante hinausragt. Dies erlaubt dem Taper eine angenehme Arbeitshöhe.

Wenn kein Behandlungstisch zur Verfügung steht, z.B. am Spielfeldrand, so kann der Unterschenkel sitzend über einem Behandlungskoffer in Position gebracht werden.

Es ist wichtig, dass der Athlet das Bein gestreckt und in extremer Dorsalflexion hält. Hierdurch wird verhindert, dass der Verband zu eng angelegt wird.

Der Taper steht gebeugt vor dem ihm entgegen gestreckten Bein. Nur so kann er flexibel und schnell aus allen Blickwinkeln den Verlauf des Tapes bestimmen und verfolgen. Die sitzende Position ist für den Taper tabu.

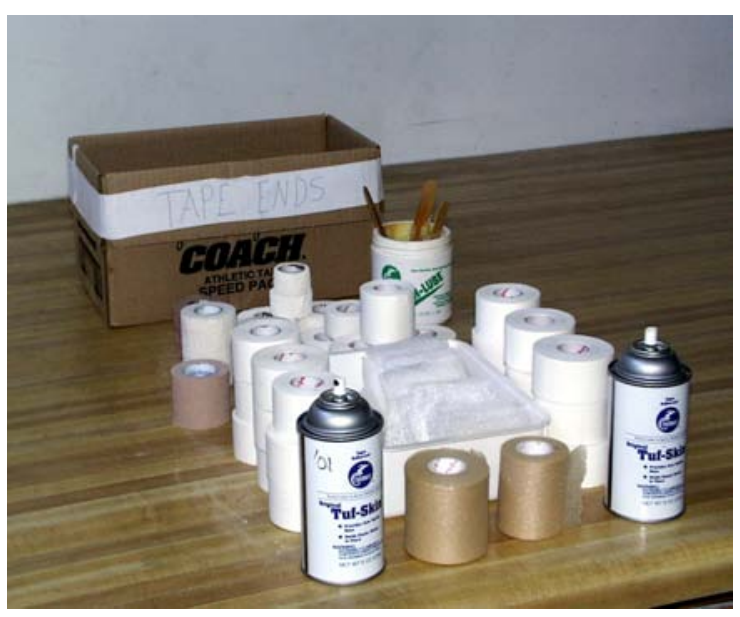

Aus der großen Auswahl an Materialien, empfiehlt sich Tape aus Baumwolle mit feiner Perforation. Dieser ist nicht nur atmungsaktiver, sondern erlaubt auch ein leichteres und gleichmäßigeres Entrollen des Tapes von der Spule. Es hat sich 
erwiesen, dass Tape mit einer Spule aus Pappe auch an schwitzenden Fingern des Tapers nicht haften bleibt und somit viel Zeit ersparen kann.

Das Tape wird grundsätzlich ohne Zug angebracht. Das Gelenk wird in gewünschter Position gehalten und das Tape angelegt. 
Wie schon erwähnt, gibt es eine Vielzahl an Möglichkeiten ein Gelenk zu tapen. Es soll nun die im Rahmen dieser Arbeit durchgeführte Technik beschrieben werden:

2.4.3.1 Technik

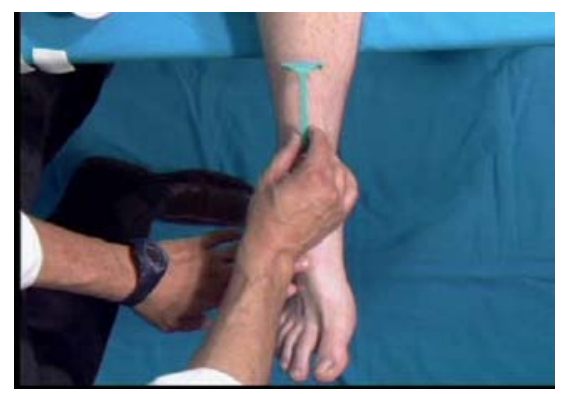

Auf den rasierten Unterschenkel des Springers wurde zunächst ein feiner Klebefilm gesprüht, um einerseits die Haftung des Tapematerials mit der Haut zu optimieren und andererseits,

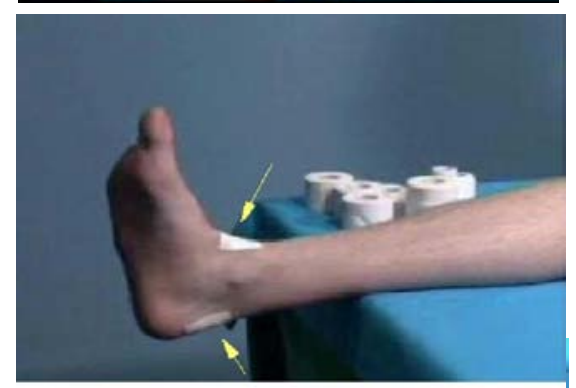

um die Entfernung des Tapes im Nachhinein zu erleichtern und Hautirritation zu mindern. Dann wurden zum weiteren Schutz der hervortretenden Sehnen der Fußheber, sowie über der Achillessehne je ein klebendes Polster angebracht.

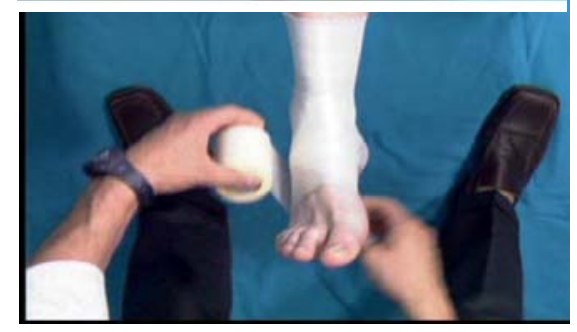

Es folgte dann die Anlage des Vorverbandes, dem so genannten Pre-Wrap. 
Dann wurden proximal des Sprunggelenkes zwei Anker und distal am Vorfuß ein Ankerstreifen angelegt. Diese verliefen halb überlappend auf der Haut des Athleten (s.Abb.). Es folgte dann beidseits je ein zirkulär aufsteigender Zügel, hier „Hogie-Strip“ genannt, welcher die Ferse an Vorfuß und Unterschenkel stabilisiert (s.Abb.). Als nächstes wurde, in der Form eines Steigbügels, von lateral beginnend ein Streifen vom Unterschenkelanker, über die Ferse nach medial hoch zum Unterschenkelanker gelegt. Hierbei wurde der Fuß angedeutet in Supinationsstellung gehalten (nicht gezogen!), um der beim Absprung auftretenden

Pronation entgegenzuwirken. Dann wurde in der Form eines Hufeisens, ein Zügel vom medialen Vorfußanker, über den Ansatz der Achillessehne nach lateral zum Vorfußanker geführt, um die beim Absprung entstehende Außenrotationstendenz des Vorfußes zu
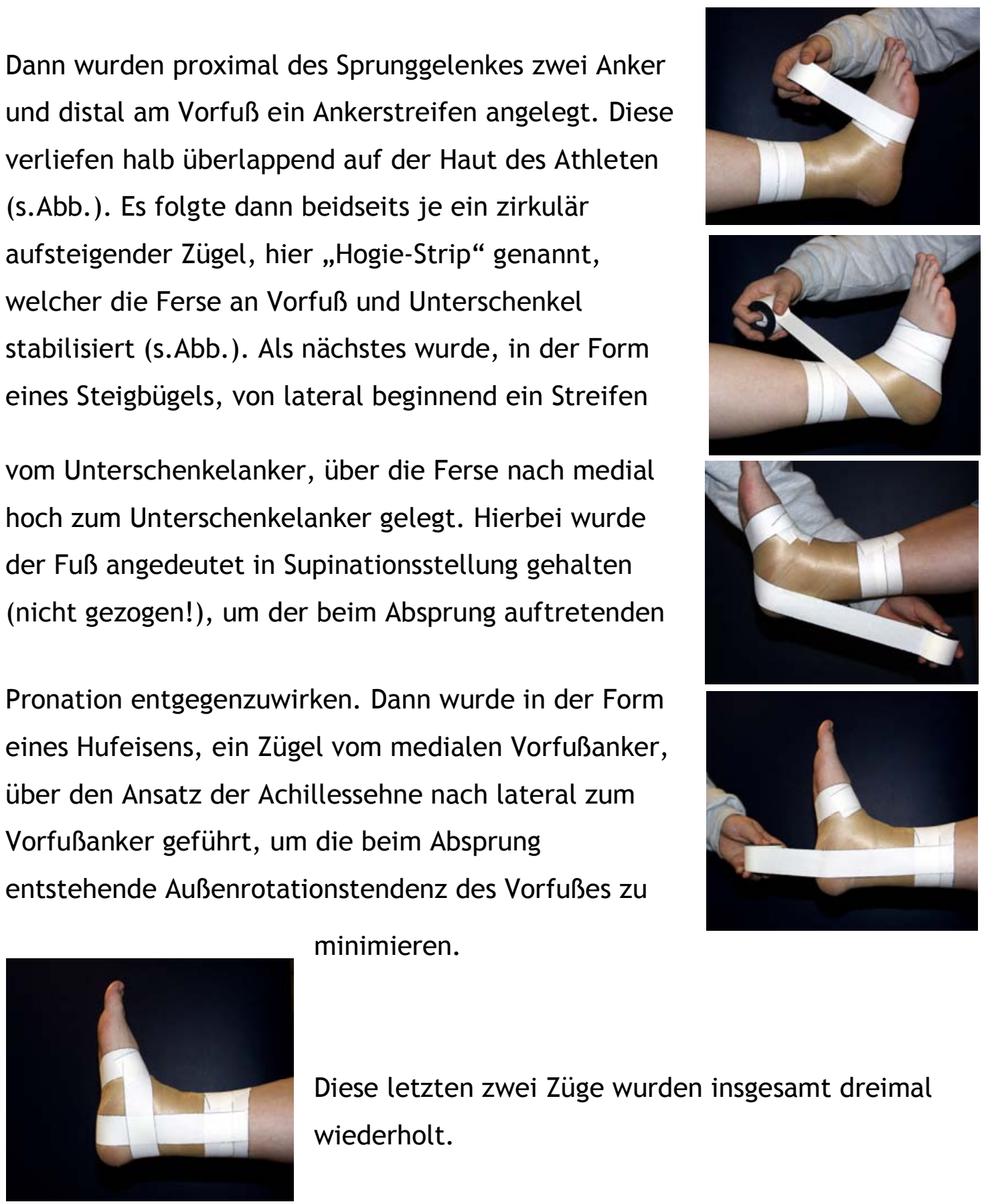
minimieren.

Diese letzten zwei Züge wurden insgesamt dreimal wiederholt. 


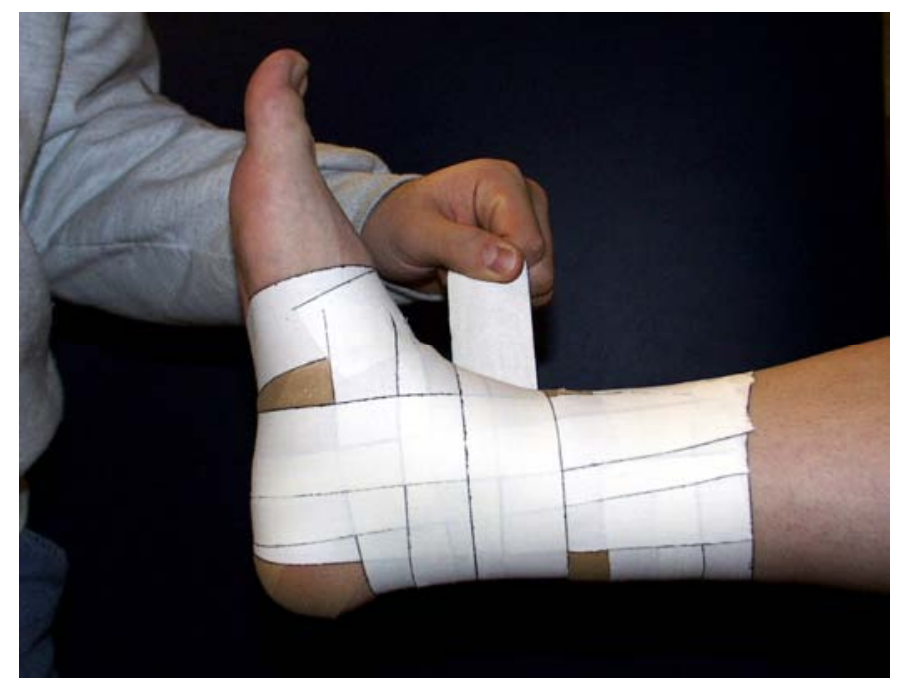

Dabei überlappten sich die Zügel um $1 / 2$ bis $1 / 3$, sodass die letztendliche Formation an eine Korbflechterei erinnert (s.Abb.).

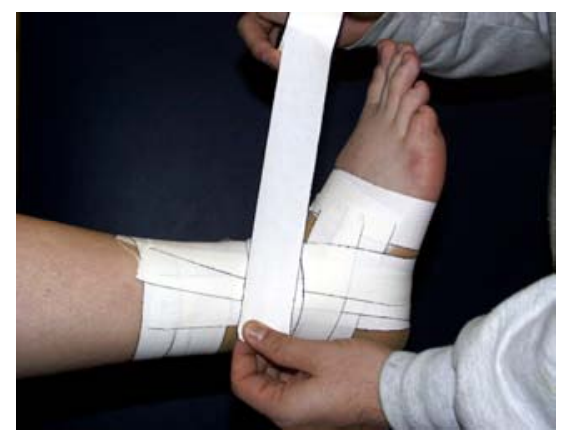

Die nächste Figur wird „Achterfigur“ genannt und dient zur Stabilisierung des Mittel-/Vorfußes am Unterschenkel.

Hierzu wurde proximal des lateralen Malleolus

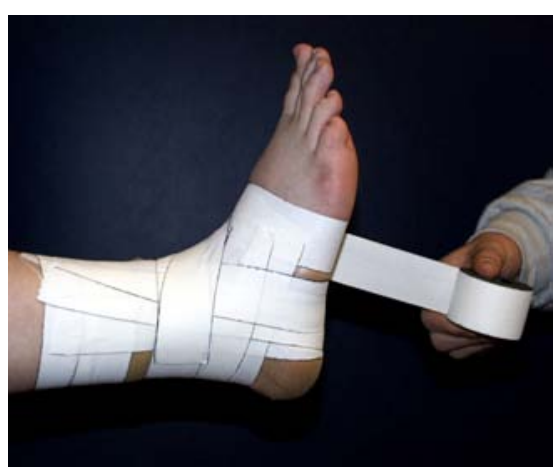
beginnend, das Tape nach vorne medial unter die Sohle geleitet und nach lateral wieder herauf, um seinen Lauf nach medial um den distalen Unterschenkel, knapp über den Knöcheln, fortzusetzen, um dann schliesslich über den lateralen Knöchel wieder zum Ausgangspunkt zurückgeführt zu werden (s.Abb.).

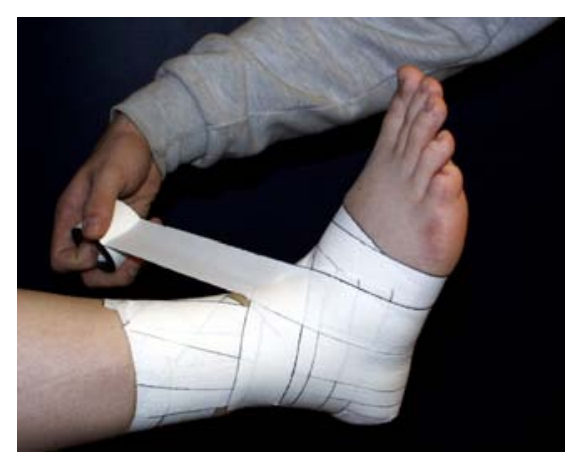




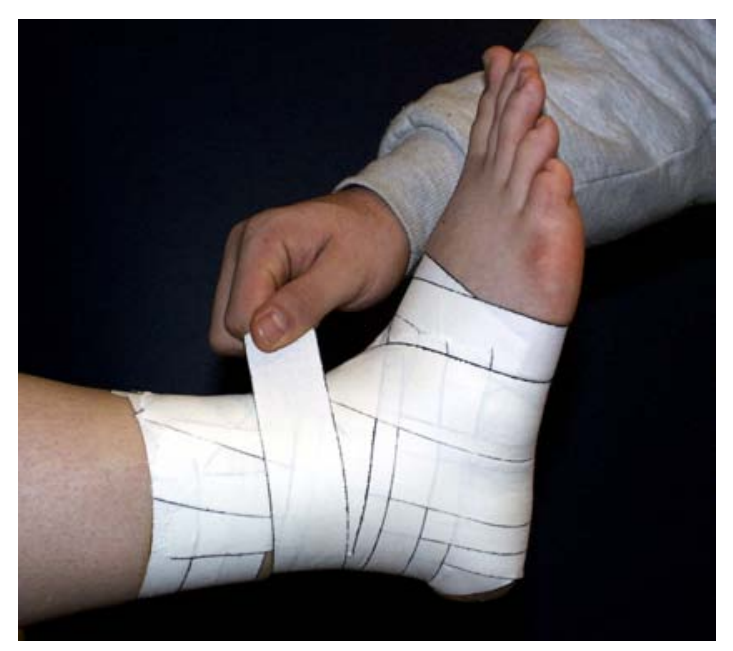

Diese Figur kann, je nach Gewicht und Belastung geradezu beliebig oft ohne Abriss wiederholt werden.

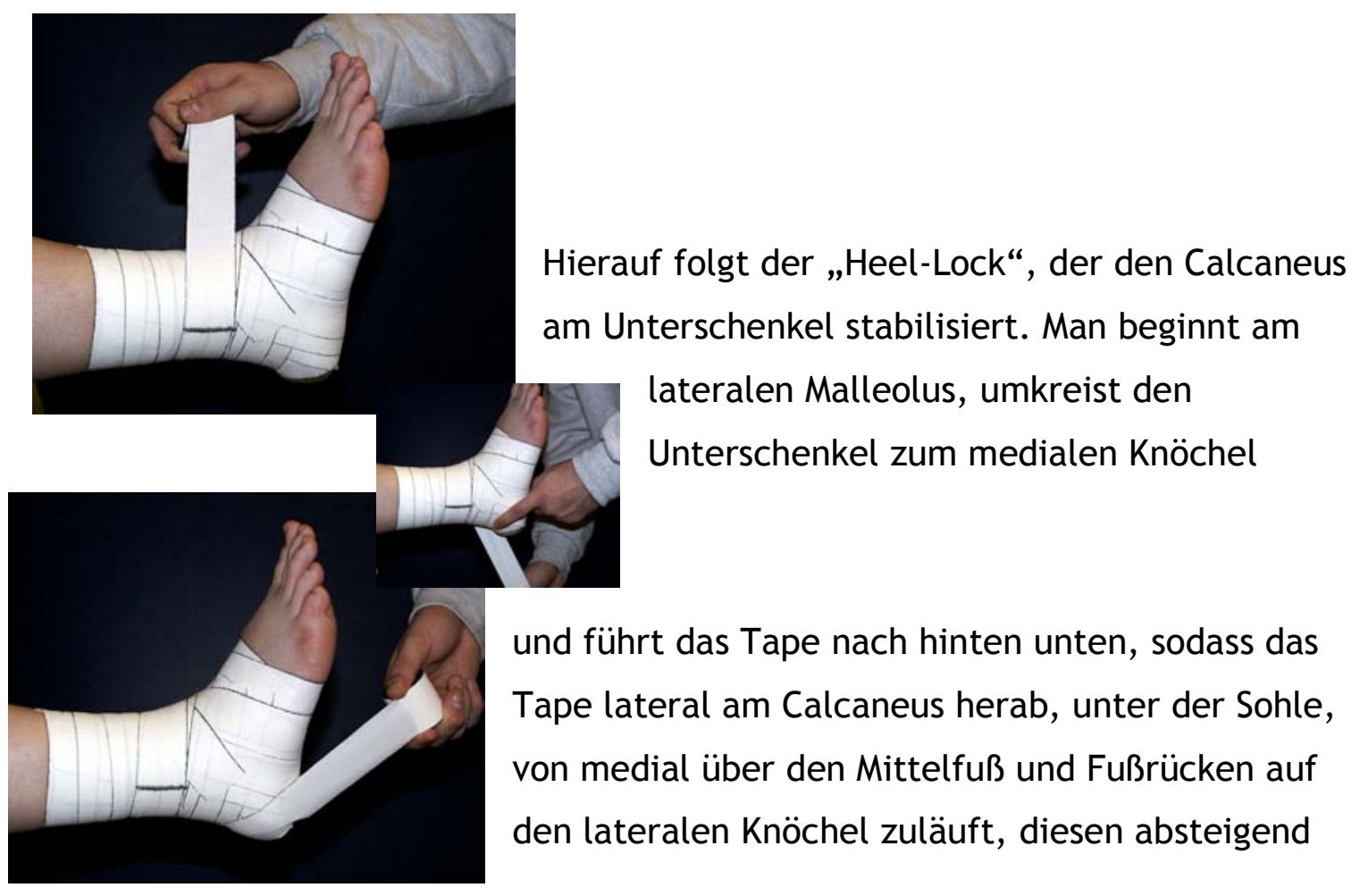




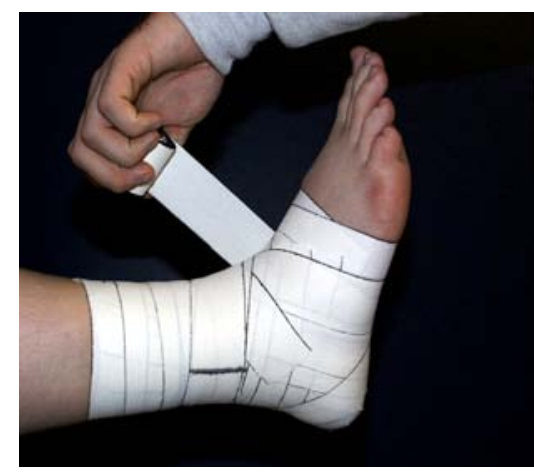

überquert, um medialseitig am Calcaneus herab zu laufen. Das Tape nimmt dann seinen Weg unter der Sohle lateralwärts und endet am Ausgangspunkt im Übergang zwischen Mittelfuß und medialem Knöchel. Auch diese Figur kann beliebig oft aneinander gereiht

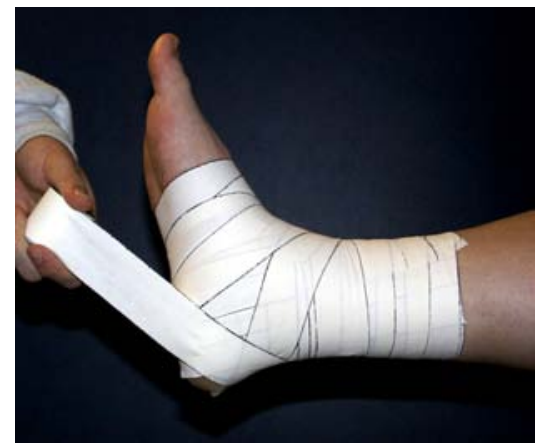
werden.
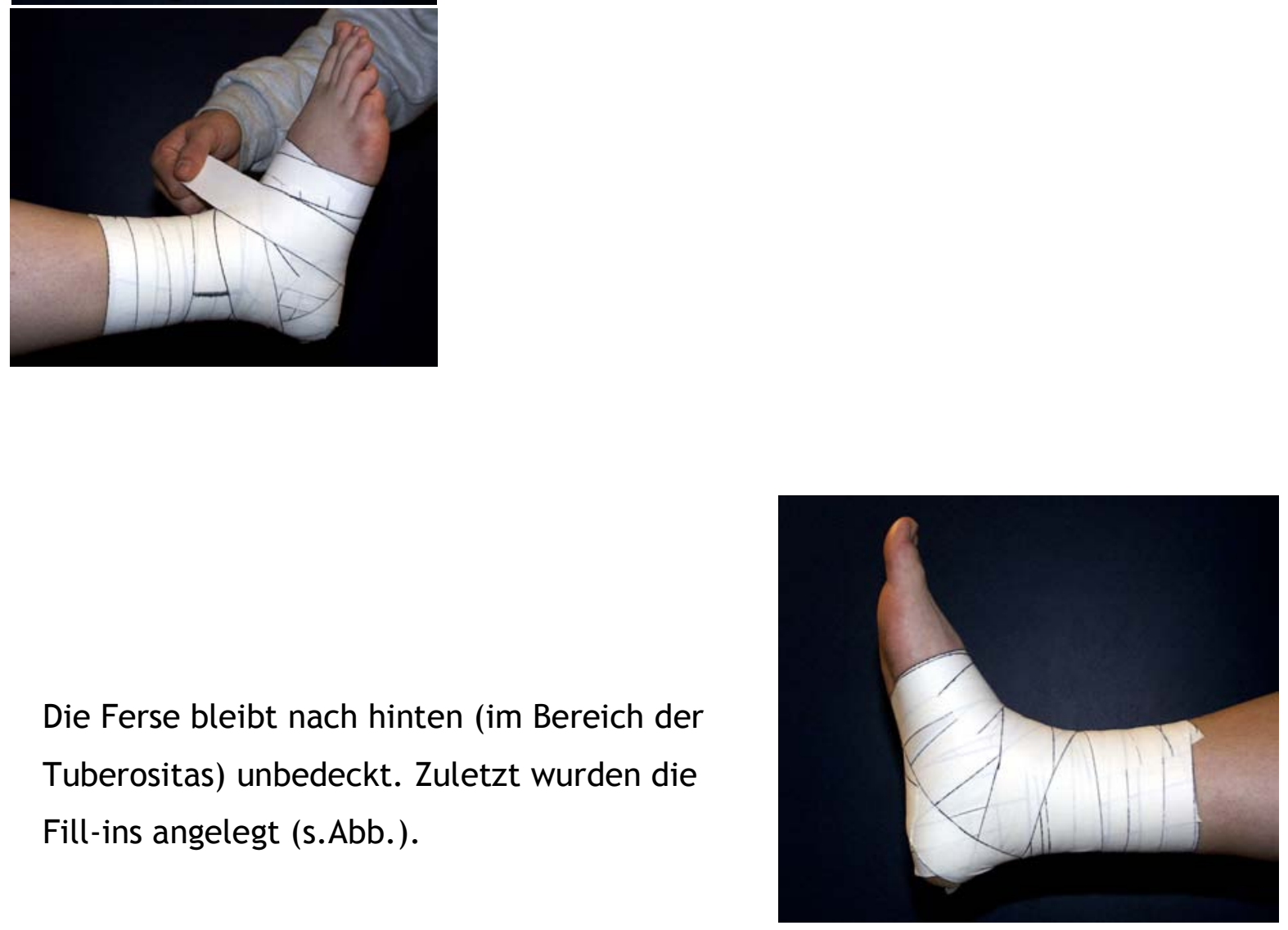

Die Ferse bleibt nach hinten (im Bereich der Tuberositas) unbedeckt. Zuletzt wurden die Fill-ins angelegt (s.Abb.). 


\subsubsection{Biomechanik}

Wenn der Tapeverband richtig angelegt ist, wird die unerwünschte Bewegungsebene eingeschränkt, während den betroffenen Strukturen noch genügend Bewegungsfreiheit bleibt um insgesamt funktionell zu bleiben. Ein verletztes oder verletzungsanfälliges Gelenk wird durch Tape überbrückt, indem die beteiligten anatomischen Strukturen in ihrem Verlauf nachgeahmt werden. Das oben erwähnte Pre-Tape soll das Entfernen des Tapematerials nach Gebrauch erleichtern. Meist wird, um den Kontakt und somit die Stützfunktion, zwischen Tape und Körper zu optimieren, ein klebriger Kontaktfilm auf die Haut gesprüht. Dies soll die Kraftübertragung zwischen Material und Bewegungsapparat verbessern. Das betroffene Gelenk sollte in einer Position gehalten werden, die der Bewegung des Verletzungsmechanismus entgegen sitzt. Beispielsweise, sollte bei Zustand nach einer Supinationsverletzung des Sprunggelenkes der Fuß zum Tapen in einer angedeuteten Pronationsstellung gehalten und dann das Tape angelegt werden. Es dabei unbedingt darauf zu achten, dass das Tape nie unter Zug angebracht wird, sondern immer nur angelegt wird. Eine Standardtechnik gibt es nicht. Um den unterschiedlichen biomechanischen Verhältnissen funktionell gerecht zu werden, sind Tapeverbände in ihrer Anlage und Technik individuell an Anatomie, Verletzung und/oder Sportart bzw. Spielposition anzupassen. Natürlich ist sauberes Arbeiten mit Vermeidung von Falten und Lücken absolut unerlässlich, nicht zuletzt auch um Sekundärverletzungen, wie Blasen, Schnittund Schürfwunden durch das Tragen des Tapes, zu vermeiden. 


\section{Fragestellung}

Mit dieser Arbeit soll untersucht werden, ob und inwiefern ein Tapeverband am Sprunggelenk von Hochspringern tatsächlich eine verbesserte Leistung gewährleistet und somit als fester Bestandteil der Ausrüstung zur Technikoptimierung und Leistungssteigerung beiträgt. Es sollen die einzelnen beteiligten Faktoren eruiert und, sowohl isoliert, als auch im Gesamtgefüge der Disziplin, analysiert und in ihrer Wichtigkeit dargelegt werden. 


\section{Methodik}

\subsection{Material und Untersuchungsgut}

Es wurden insgesamt 14 Hochspringer/innen (8 Männer und 6 Frauen) im Alter zwischen 16 und 27 Jahren untersucht. Als Einschlusskriterium galt eine persönliche Bestmarke von mindestens $170 \mathrm{~cm}$ bei den Frauen, sowie eine von mindestens $195 \mathrm{~cm}$ bei den Männern. Die Athleten wurden hierzu bei Meisterschaften auf Landesebene des Freistaates Bayern rekrutiert. Außerdem wurden 21 Breitensportler zur Untersuchung der Teilaspekte des Hochsprungs, wie Stabilität, Propriozeption, Kraftentwicklung, Gleichgewicht, usw. herangezogen. Alle Probanden wurden mit Fußdruckmesssohlen, sowie einem Transpondermodem (Sender) der Firma T\& T Medilogic ( $)$ ausgestattet. Diese wurden während der Untersuchungen als Einlegesohle bzw. an einem Gürtel befestigt am Körper getragen. Der Computer empfing die Messdaten über ein Empfängermodem (s. Abb.).

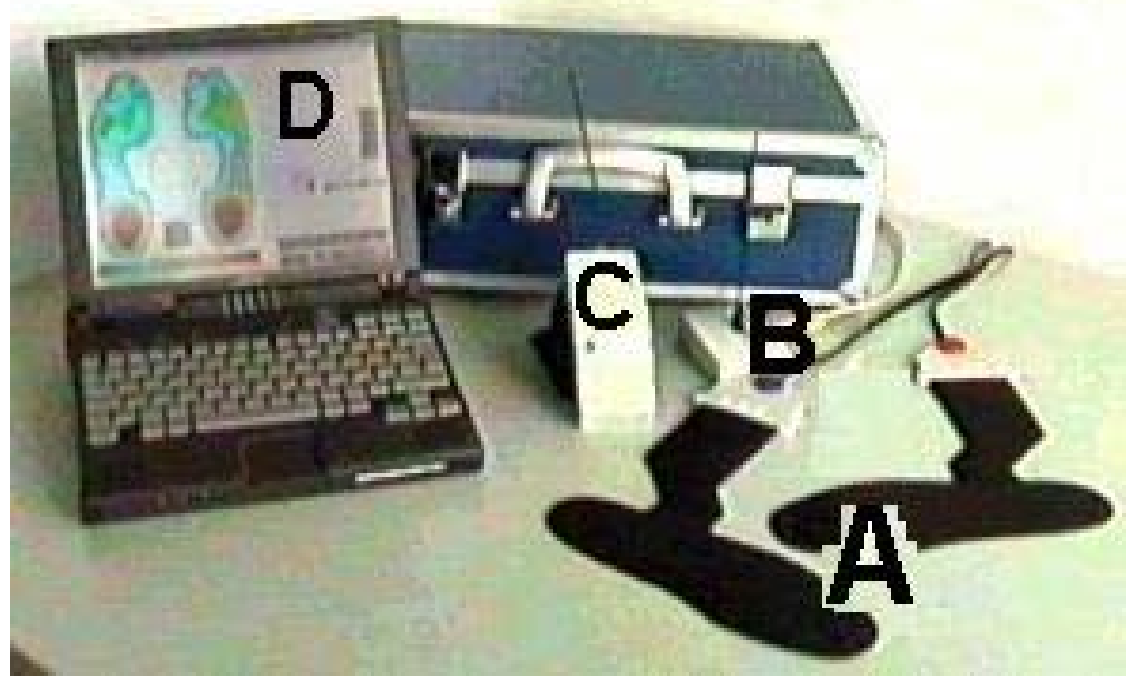

[Abb.- - A - Meßsohlen, B - Transponder, C - Empfänger, D - Computer] 
Hier wurden sie bearbeitet und analysiert. Die Messungen wurden jeweils mit und ohne Tapeverband dokumentiert und miteinander verglichen. Um den Vorteil eines Aufwärmeffektes zu neutralisieren, wurden zwei Gruppen erstellt, in denen die Messungen entweder zunächst mit Tapeverband oder zunächst ohne Tapeverband durchgeführt wurden. Die Probanden wurden randomisiert einer der zwei Gruppen zugeordnet. Aus dem komplexen Gesamtgefüge des Hochsprungs wurden die Hauptfaktoren Propriozeption, Stabilität und Beweglichkeit isoliert, in sechs Versuchen, untersucht.

\subsubsection{Das Medilogic Fußdruckmeßsystem}

Das telemetrische Medilogic Fußdruckmeßsystem ermöglicht eine kabellose Messung der auftretenden statischen (im Stand) und dynamischen (während des Sprunges) Druckbelastungen am Fuß des Athleten. Die Druckwerte im Schuh werden direkt über Funk zum Computer gesendet und dort am Monitor angezeigt. Eine sofortige Sichtkontrolle während der Messung ist damit möglich, ohne dass lästige Kabelverbindungen die Bewegungsfreiheit einschränken. Die Funkübertragung des Systems befindet sich im so genannten IMS- Band, einem Frequenzbereich, der Europaweit für „INDUSTRIE-SCIENCEMEDICAL“ Bereiche vorgesehen ist und $433,92 \mathrm{MHz}$ bzw. 2,4 GHz beträgt. Die Reichweite betrug ca. 100 Meter im Freien und 25 Meter im Gebäude.

Dieses System wird eigentlich hauptsächlich im Bereich der Orthopädie genutzt, wo nach Erfassung der Belastungsverteilung an der Fußsohle beim Gehen und Stehen, die Ausgangsdaten für die Herstellung, Anpassung, Kontrolle und Dokumentation von Schuhversorgungen herangezogen werden. Die in dem 
Bereich eingesetzten Systeme arbeiten mit einer Meßrate von $60 \mathrm{~Hz}$. Das in dieser Arbeit eingesetzte System bietet eine Meßrate von $300 \mathrm{~Hz}$, welche für eine ausreichend detaillierte Messung im Hochsprungbereich eingesetzt werden kann.

Das System besteht also aus Druckmesssohlen, die in Paaren von insgesamt acht Schuhgrößen geliefert wurden. Die Messsohlen waren mit je 64 oberflächenresistive Sensoren ausgestattet, wurden in die Schuhe des Athleten eingelegt und über Kabel, die entsprechend entlang der Beine fixiert wurden, an ein Modem verbunden. Dieses Modem war an einem Gürtel seitlich an der Hüfte befestigt. Ein zweites Modem in der Nähe des Computers empfing die Messdaten und leitete sie wiederum über ein Kabel an den Computer.

Die unter WINDOWS ® laufende Medilogic Software ermöglicht eine Sofortanzeige der Messwerte auf dem Computermonitor. Die Druckverhältnisse können wahlweise als dreidimensionales farbiges Druckgebirge oder als zweidimensionale Farbgrafik dargestellt werden.

Die Bedienung von Medilogic ist vergleichbar mit der eines Video-Recorders. Per Mausklick wird die Messung (Daten-Aufnahme) gestartet. Dies erlaubte den Komfort, unmittelbar nach Beendigung der Aufnahme das Resultat der Messung entweder in Originalgeschwindigkeit, im Schnelldurchlauf oder in Zeitlupe anzuwählen und zu betrachten.

Auch die Darstellung von Druckmaxima, Impulswerten und Durchschnittswerten war möglich. Der Verlauf vom Körperschwerpunkt über den Sohlen, in Ganglinie und Zyklogramm ist ebenfalls möglich gewesen. 
Der Vergleich zweier Messungen kann durch direkte Gegenüberstellung auf einem Screen durchgeführt werden.

Einzelbilder (Screenshots) konnten mit Hilfe des Programms MGI PHOTO SUITE ${ }^{\circledR}$ von SONY $®$ zur Einfügung in diesen Text importiert werden.

Zur Archivierung der Daten konnte jede Messung einem Athleten zugeordnet werden und auf Festplatte gespeichert werden. Ein späteres Auswerten war somit jederzeit möglich. Über eine umfangreiche Datenpflegefunktion ließen sich die Daten sichern, exportieren und bei Bedarf wieder importieren. 


\subsubsection{Der POSTUROMED ${ }^{\circledR}$ Schwingboden}

Dieses Gerät aus dem Hause HAIDER BIOSWING besteht aus einer Plattform, welche frei schwingend an Gummiriemen aufgehängt ist und die Beweglichkeit in zwei Ebenen zulässt.

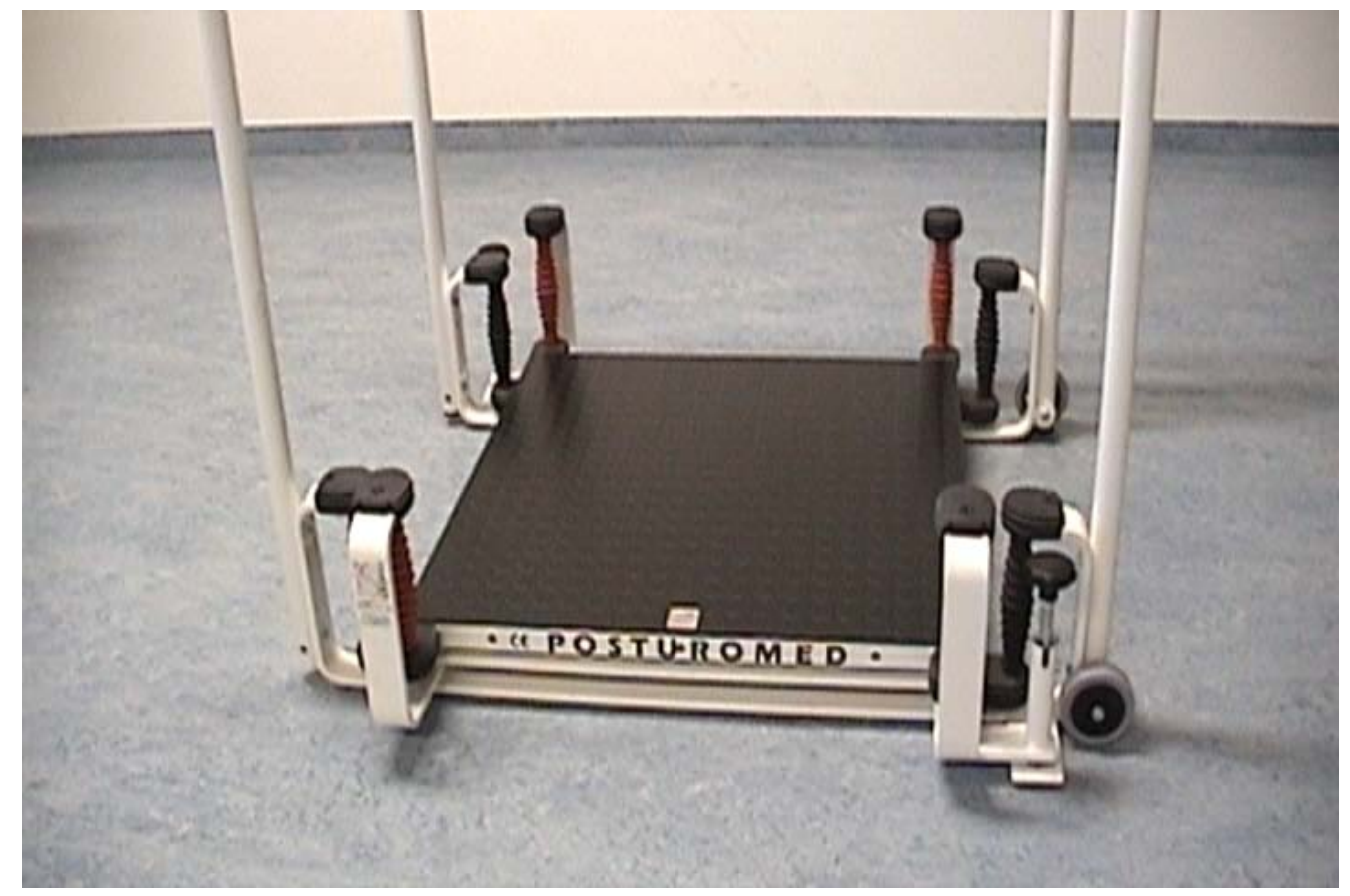

Dieses Gerät ist eigentlich als sensomotorisches Trainingsgerät mit dem Prinzip der rhythmischen Stimulation zum Training von Gleichgewicht, Koordination und Stabilität, sowie zum Harmonisieren des Zusammenspieles der Muskulatur von Rumpf und Extremität konzipiert worden. Hiermit wird besonders die axiale segmentale Koordination, die wichtig für eine aufrechte Körperhaltung ist, trainiert. Der Posturomed $®$ schwingt in allen Richtungen und das Halten des Gleichgewichtes ist äußerst schwierig. Koordination und Gleichgewicht werden hier ganz besonders auf die Probe gestellt. 
In der Klinik wird POSTUROMED typischerweise bei den folgenden Indikationsstellungen eingesetzt:

- funktionelle Fehlhaltung der Wirbelsäule in jedem Alter

- konstitutionelle oder lokale Hypermobilität im posturalen System

- "instabiles" Knie, "instabiles" Sprunggelenk

- idiopathische Skoliosen besonders bei Kindern und Jugendlichen

- so genannte juvenile Rundrücken

- so genannte Haltungsschwäche

- abstehende Schulterblätter (scapulae alatae)

- nach Knieoperationen, besonders nach Eingriffen an den Kreuzbändern

- Störungen des Fußgewölbes, besonders bei Kindern

- inkomplette, schlaffe Lähmungen im posturalen System

- Polyneuropathien, wo Störungen der propriozeptiven Afferenz überwiegen

Wir haben das Gerät bei Versuchen des Gleichgewichts und somit der Propriozeption herangezogen. 


\subsubsection{Der Therapiekreisel}

Der Therapiekreisel ist eine runde Holzplatte mit einer Hemisphäre an der Unterseite, deren Konvexität zum Boden Kontakt hält. Bei dem hier verwendeten Modell ist die Marke nicht erkenntlich gewesen.

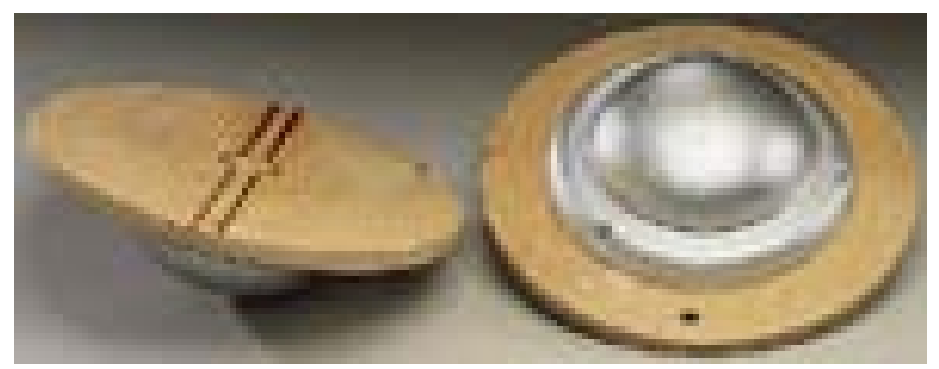

Auch dieses Gerät wird gewöhnlich in der Rehabilitation nach Verletzungen, insbesondere des Sprung- und / oder Kniegelenkes, eingesetzt. Es dient der Beschleunigung der Rückgewinnung von Propriozeption und Sicherheitsgefühl in der verletzten Extremität, aber auch entlang der gesamten Bewegungskette.

Patienten benutzen dieses Übungsgerät, indem sie darauf im Einbeinstand das Gleichgewicht zu halten versuchen.

Wir haben es in unseren Versuchsreihen zur Beurteilung des Gleichgewichtssinnes eingesetzt. 


\subsection{Versuchsreihen}

\subsubsection{Propriozeption}

\subsubsection{Versuch 1: Dominanz / Dextrosität}

Zur Ermittlung der Dominanz der unteren Extremität wurde jeder Proband aufgefordert, das Körpergewicht im Stand nach subjektivem Empfinden gleichmäßig auf beide Beine zu verteilen. Die dabei empfangenen Daten vermittelten auch die Gewichtsverteilung und somit das Lot des Körperschwerpunktes (KSP) in Relation zur Standfläche. Dann wurden beidseits die Sprunggelenke getaped und die Messungen erneut erhoben. Diese Information sollte Aufschluss darüber erbringen, ob es

1. eine Korrelation zwischen der ermittelten Dominanz und der Dextrosität gibt und ob es

2. durch Anlage eines Tapeverbandes zu einer Verschiebung des KSP als Zeichen einer veränderten propriozeptiven Wahrnehmung.

Ferner stellten diese erbrachten Daten Ausgangswerte dar, mit denen die während der Versuche „Temperatur“ gewonnen Daten verglichen werden sollten. 


\subsubsection{Versuch 2: Temperatur}

Inwiefern eine erhöhte bzw. verminderte Propriozeption des Fußes zu einer Minder- oder Überbetonung desselben führt, wurde mittels Abkühlung des einen bzw. Erwärmung des anderen Fußes einschließlich Sprunggelenk und Sohle ermittelt.

Zur Abkühlung verwendeten wir die in der Physikalischen Therapie üblichen EisPackungen, welche wir auf minus $18^{\circ} \mathrm{C}$ gekühlt für exakt 10 Minuten unter, auf und um den zu kühlenden Fuß legten und mit vier 2 kg Sandsäcken jeweils von medial und lateral, sowie von hinten gegen die Achillessehne und über dem Fußrücken, stabilisierten.

Zur Erwärmung des Fußes benutzten wir die in der Physikalischen Therapie üblichen Fango-Packungen, welche auf $60^{\circ} \mathrm{C}$ vorgeheizt unmittelbar in gleicher Manier angelegt und stabilisiert wurden. Ziel war es eine deutliche Temperaturdiskrepanz zwischen rechtem und linkem Fuß zu erzeugen.

Die Probanden wurden, ungeachtet ihrer Dominanz bzw. Dextrosität, randomisiert zwei Gruppen, RK und RW, zugeteilt. In der Gruppe RK wurde der rechte Fuß abgekühlt, während der linke erwärmt wurde. Gruppe RW erfuhr die Umkehrung der RK-Prozedur. 


\subsubsection{Versuch 3: Gleichgewicht und Stabilität}

Versuch 3a:

Der Verlauf des Körperschwerpunktes über der Fußsohle wurde im Einbeinstand bei geschlossenen Augen (Wegnahme des optischen Wahrnehmung) gemessen. Hierbei wurden die Probanden aufgefordert, während des Beidbeinstandes die Augen zu schließen und dann in den Einbeinstand der Wahl überzugehen. Um Hilfsbewegungen anderer Extremitäten auszuschließen, wurden die Hände hinter dem Körper verschränkt und der Fußrücken der Gegenseite hinter der Achillessehne des getesteten Beines verschränkt. Die Probanden standen in Knie und Hüftgelenk leicht flektiert, mit dem Oberkörper bei neutral gestellter Wirbelsäule leicht vorgeneigt. Ein Versuch wurde als erfolgreich gewertet, wenn es zu keinem Aufsetzen des Gegenfußes auf den Boden kam. Der Einbeinstand sollte für 30 Sekunden gehalten werden. 
Versuch 3b:

Ähnlich wie bei der unter $3 a$ beschriebenen Versuchsdurchführung, wurde auch bei diesem Versuch der Proband aufgefordert für 30 Sekunden im Einbeinstand das Gleichgewicht auf der Posturomed ${ }^{\circledR}$ Plattform, zu halten. Da mit diesem Gerät hauptsächlich der Einfluss der Stabilitätskomponente des Tapes auf die Koordination erfasst werden sollte, wurde der Versuch mit offenen Augen durchgeführt, um Koordinationsmängel zu minimieren. Ein Versuch wurde als erfolgreich gewertet, wenn es zu keinem Aufsetzen des Gegenfußes kam.

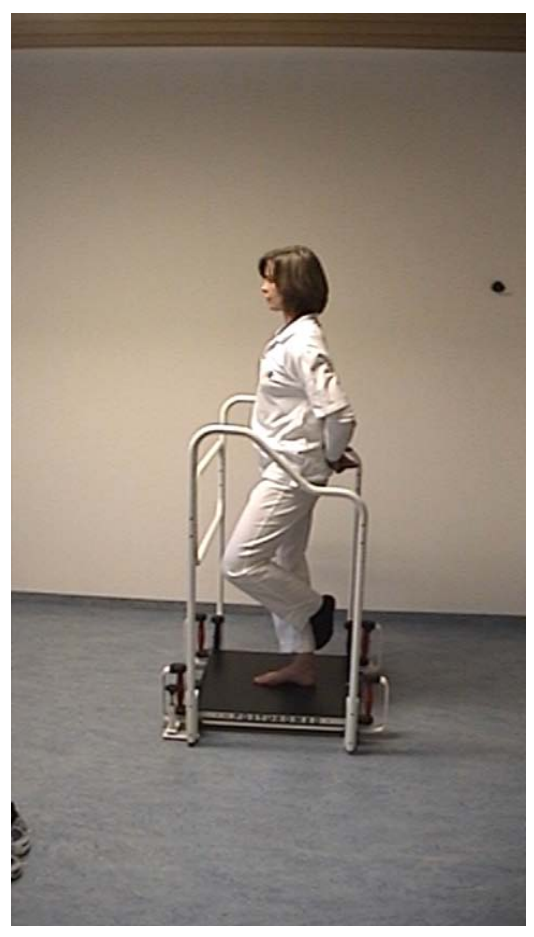

Versuch 3c:

Schließlich wurde der Versuchaufbau, ebenso mit offenen Augen, auf einem Therapiekreisel durchgeführt. Ein Versuch wurde als erfolgreich gewertet, wenn es zu keinem Aufsetzen der Kreiselkante auf den Boden kam. Der Einbeinstand sollte für 30 Sekunden gehalten werden.

Während aller drei Versuche wurde der Verlauf des KSP-Lotes über der Sohle aufgezeichnet und den Vergleichsdaten mit Tape gegenübergestellt.

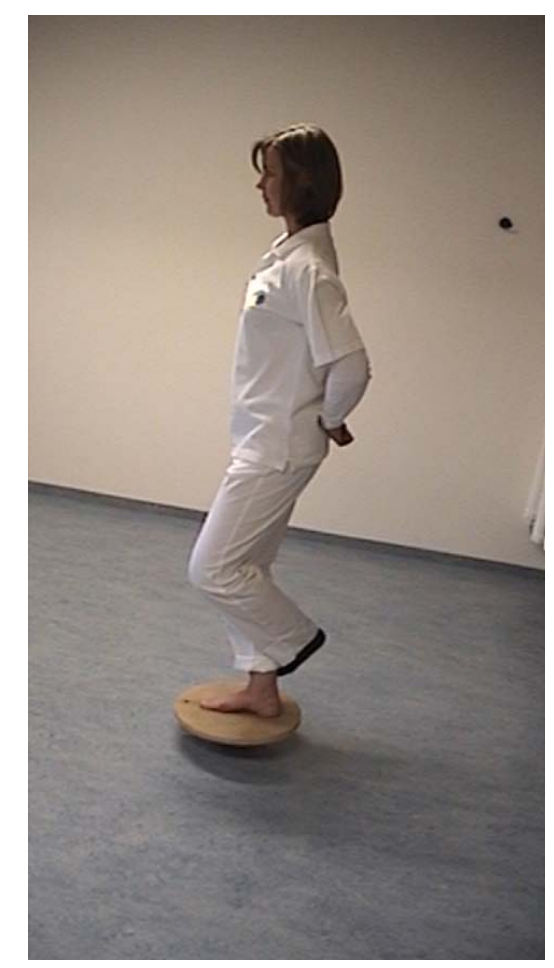




\subsection{Leistung}

\subsubsection{Versuch 4: Sprunghöhe}

Aus den digitalen Aufnahmen

während des Hochsprunges ging

hervor, dass der Einsprungwinkel in

die Stemmphase des letzten

Bodenkontaktes vor der

Lattenüberquerung ca. $35^{\circ}$ zum

Boden betrug. Letztendlich bestimmt

die Zuversicht und das

Selbstvertrauen des Sportlers das

Tempo und somit diesen

Einsprungswinkel. Diese Phase des

Absprunges sollte simuliert werden, um den Einfluss der Stabilität auf das

subjektive Sicherheitsgefühl isoliert

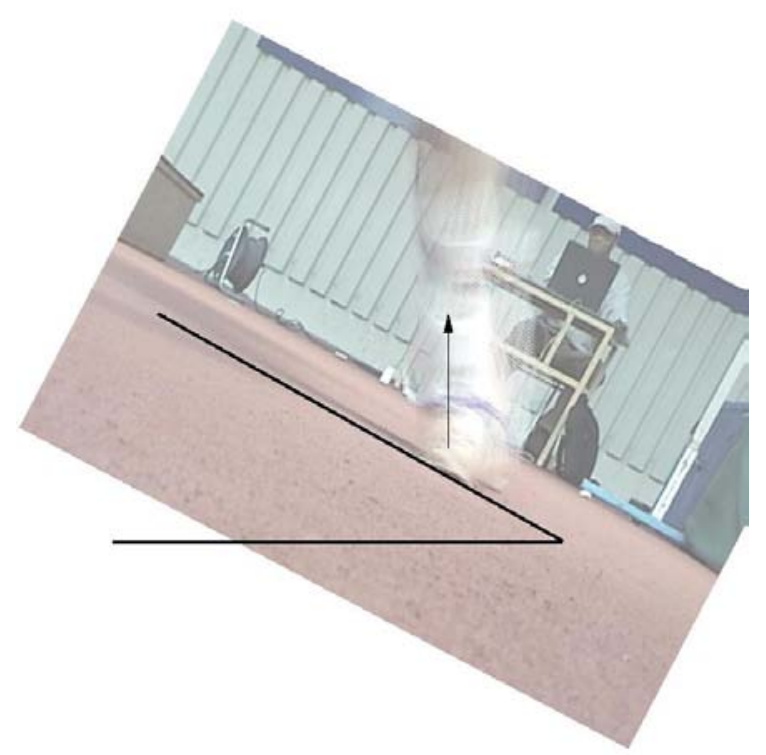

zu untersuchen. Hierzu wurden

maximale vertikale Sprünge an einer seitlichen Schräge mit $35^{\circ}$ Neigung

durchgeführt (s. Abb.). Die Sportler positionierten sich derart am Hang, dass ihr Sprungbein - im modifizierten Skifahrerjargon - das „Bergbein“ darstellte. 


\subsubsection{Sprung einbeinig am Hang}

Mit dem Schwungbein beginnend, wurden zwei Schritte Anlauf genommen. Absprung und Landung erfolgten mit dem Sprungbein an der selben Markierung am Hang. Es wurden jeweils 6 Sprünge durchgeführt, um, die Belastung beim Hochsprung simulierend, den Pronationsstress zu erfassen. Hiervon wurden die fünf höchsten Sprünge gewertet. Diese Messungen erfolgten mit und ohne Tape. Mit Hilfe der eingelegten Meßsohle konnte die Zeit zwischen Absprung und Landung auf die 1/1000stel Sekunde erfasst werden.

Die Sprunghöhe $h$ wurde errechnet durch die Formel:

$h=\left(t^{2} \times g\right) / 8$

, wobei die Flugzeit $\mathrm{t}$ sich aus $\mathrm{t}_{2}-\mathrm{t}_{1}$ berechnet und $g$ die Erdbeschleunigung ist.
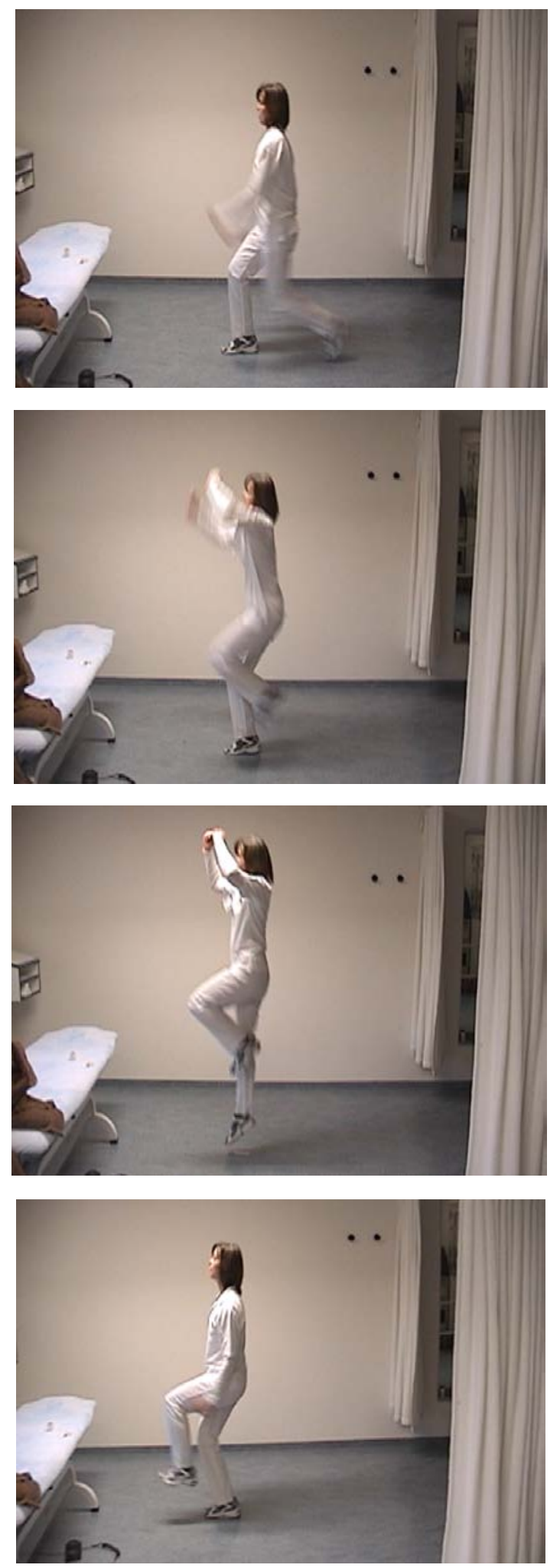


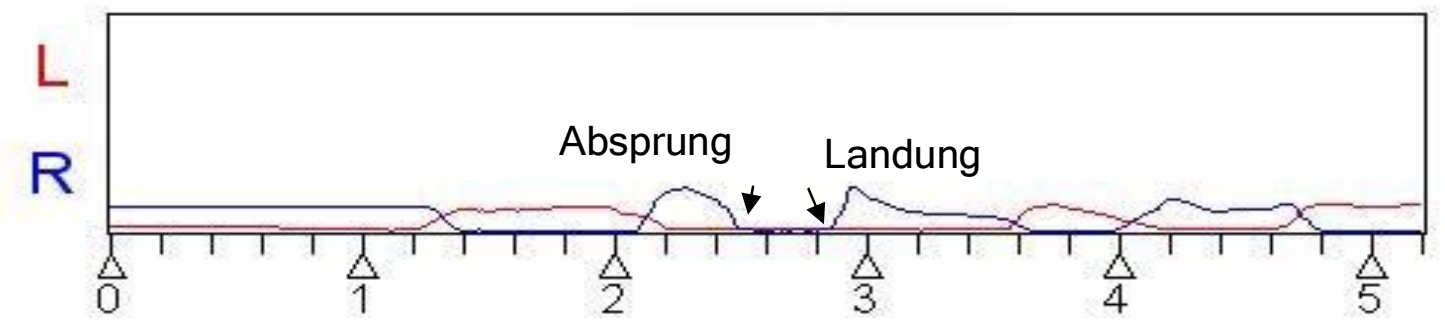

4b - Übersichtsaufnahme von Absprung und Landung, je mit bzw. auf dem rechten Bein.

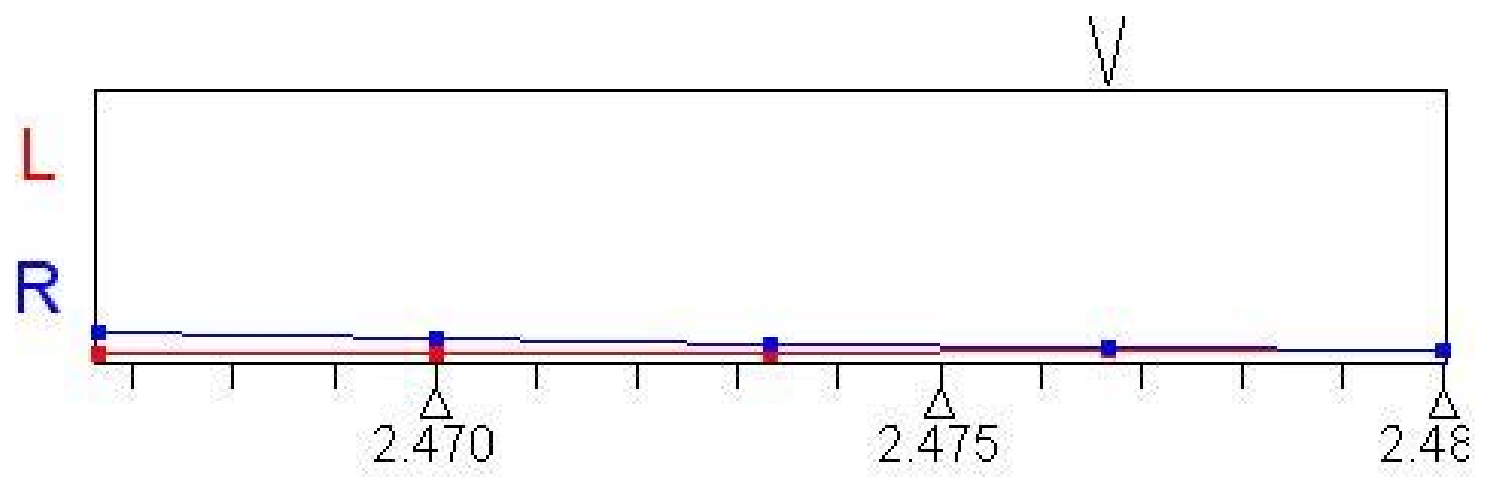

4c- Zoomaufnahme des Zeitpunktes des letzten Bodenkontaktes

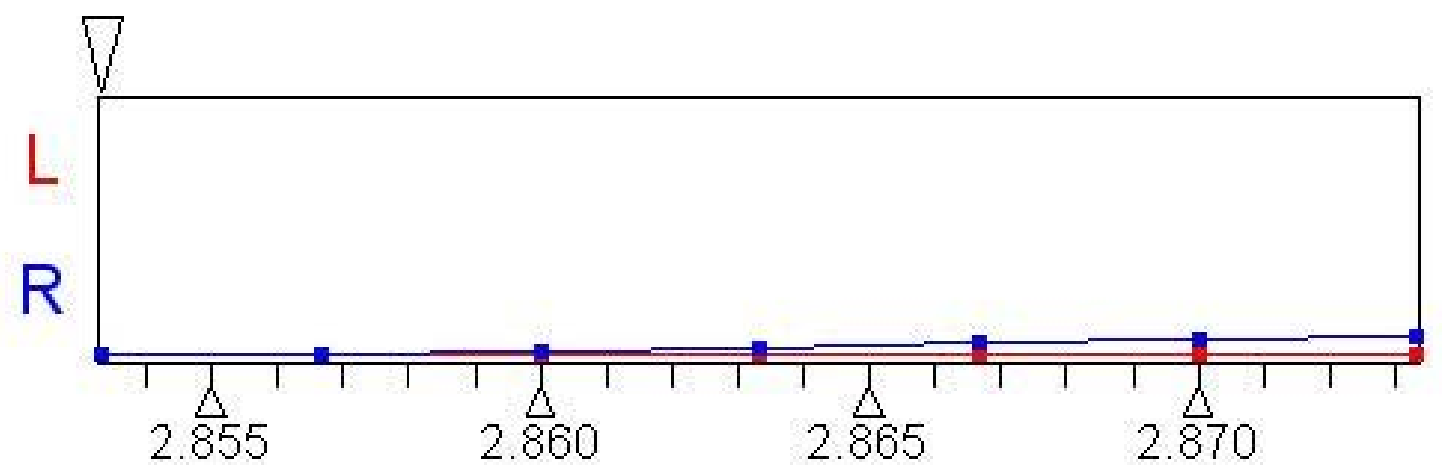

4d - Zoomaufnahme des Zeitpunktes ersten Landungskontaktes 


\subsubsection{Sprung beidbeinig auf der Ebenen}

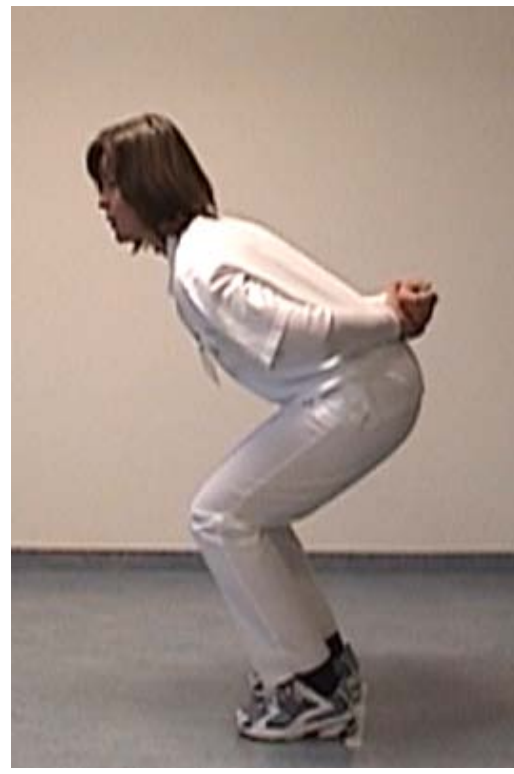

Zum Erfassen des tatsächlichen Einflusses des Tapes auf die Umsetzung der Sprungkraft, wurde der Vertikalsprung auf den „störfreien“ Minimalstandard reduziert, um Leistungsunterschie de in der intra- und intermuskulären Koordination zu neutralisieren:

Aus dem Stand wurde der Körper zunächst durch Senken des Gesäßes bis zu einem Kniebeugungswinkel von $90^{\circ}$ in die Ausgangsposition gebracht. Dann erst wurde der Sprung frei gegeben. Die Arme mussten während des gesamten Ablaufes hinter

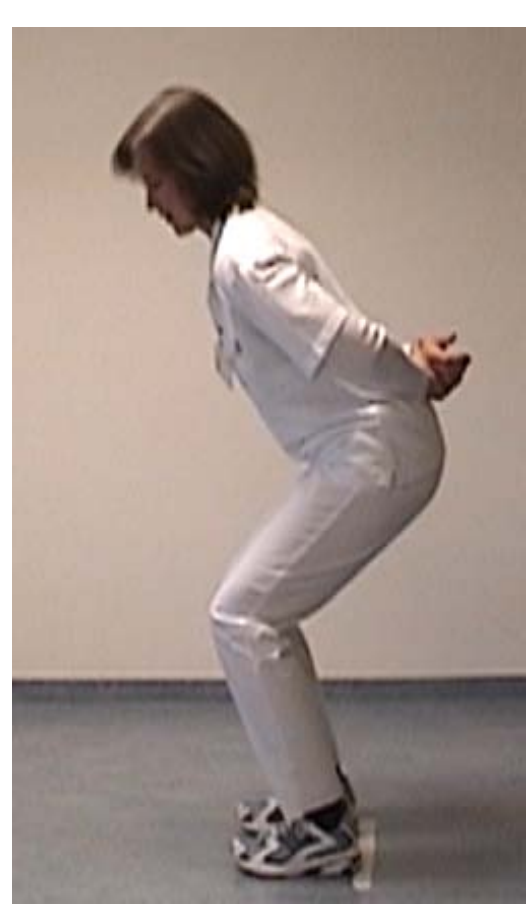
dem Körper verschränkt bleiben.

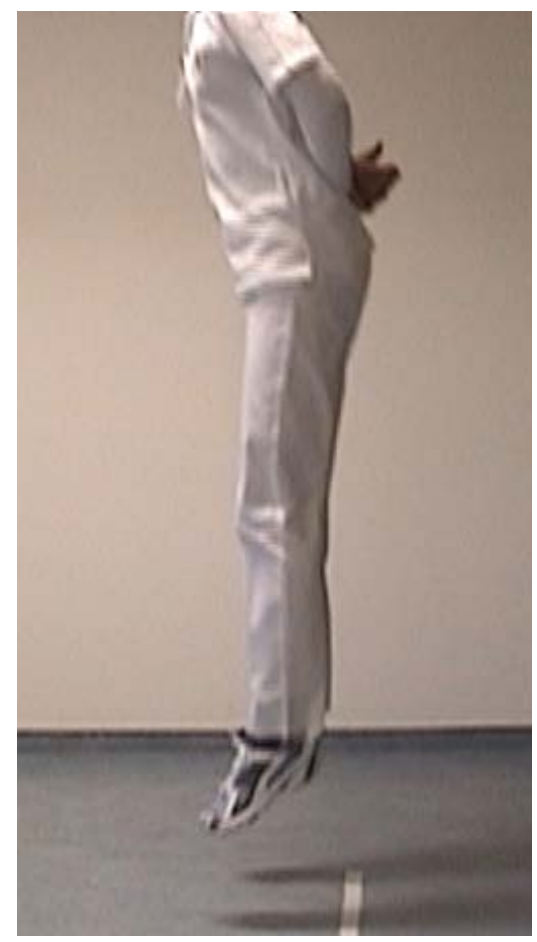

Absprung und Landung mussten eine definierte Markierung einhalten. Bei einer Abweichung von mehr als +/- 170 mm (= halbe Tapebreite) wurde der Sprung nicht gewertet. 


\subsubsection{Versuch 5: Beweglichkeit}

Bei der Untersuchung der Beweglichkeit (ROM) kommen der aktiven und passiven ROM unterschiedliche Bedeutungen zu. Hierbei betrachteten wir die zwei Bewegungsebenen des Sprunggelenkes getrennt.

In der frontalen Ebene, welche die Plantar- und Dorsalflexion zulässt, bestimmt die aktive Beweglichkeit im Sprunggelenk in großem Maß die Effektivität der Umsetzung der Geschwindigkeit in Höhe. Eine Einschränkung der Plantarflexion bedeutet eine Kürzung des Kraftweges und somit der Beschleunigungskraft. Außerdem bietet dieser letzte Bodenkontakt dem Athleten noch die Möglichkeit „letzte Korrekturen“ für eine optimale Flugbahn über die Latte vorzunehmen.

In der Pronations-/ Supinationsebene steht die Betrachtung der passiven Beweglichkeit im Vordergrund, da man zunächst annimmt, dass eine größtmögliche Stabilität in dieser Ebene für einen effektiven Sprung von Vorteil ist.

Unter standardisierten Bedingungen wurde die Sprunggelenksbeweglichkeit, zunächst ungetaped, sowie bei liegendem Tapeverband beidseits, vor und nach den jeweiligen Messreihen bzw. Sprüngen nach Neutral-Null in der Flexion/Extensions-Ebene gemessen und gegeneinander verglichen. In der Pronation/Supinations-Ebene wurde die passive Beweglichkeit nach der folgenden, eigens definierten Methode gemessen: Ein Maßband wurde unter der Ferse fixiert und lateral bzw. medial über den jeweiligen Malleolus nach proximal geführt. Es wurde eine Markierung $25 \mathrm{~cm}$ proximal des lateralen bzw. 
medialen Malleolus gesetzt. Der Proband wurde nun aufgefordert unter Belastung in die Supination bzw. in die Pronation zu gehen und die hierdurch erzeugte Streckenverlängerung zwischen Boden und Markierung wurde registriert und entsprechend verglichen. Es konnte hier so der Grad eines möglichen Stabilitätverlustes aufgedeckt werden.

Die Messreihen beinhalteten sowohl den maximalen beidbeinigen Vertikalsprung aus dem Stand, als auch den einbeinigen Vertikalsprung am Hang.

Bei der Versuchsreihe des Hochsprunges wurde vor Anlegen des Tapeverbandes sowie nach jedem Sprung eine solche Messung der ROM durchgeführt. Wir erhofften uns dadurch einen Aussagewert darüber zu gewinnen, ab wann der Tapeverband seine Effizienz hinsichtlich der Stabilität verliert.

Jede Messreihe, ob mit oder ohne Tape, stellt natürlich einen gewissen Übungslauf für die darauf folgende Vergleichsmessreihe dar. Dies wiederum könnte die Ergebnisse der zweiten Messreihen verfälschen. Um diesen Effekt als Fehlerquelle zu neutralisieren. Wurden die Probanden randomisiert in zwei Gruppen unterteilt. Die eine begann die Messreihe ohne Tape, die andere Gruppe begann sie mit Tapeverband. 


\subsubsection{Versuch 6: Der Hochsprung}

Die Hochspringer/innen wurden nach einer einheitlichen Aufwärmphase aufgefordert jeweils fünfmal die Sprunghöhe zu bewältigen, die mindestens 95\% ihrer persönlichen Bestmarke betrug. Der Absprung wurde sowohl über die Messsohle, als auch durch eine digitale Videokamera aufgezeichnet. Die Videokamera war dabei auf dem Boden im Bereich des Absprunges derart platziert, dass sämtliche Phasen des letzten Bodenkontaktes vor der Flugphase, insbesondere die Pronationsbewegung der Ferse beim Aufsetzen, von hinten dokumentiert werden konnten (s.Abb.).

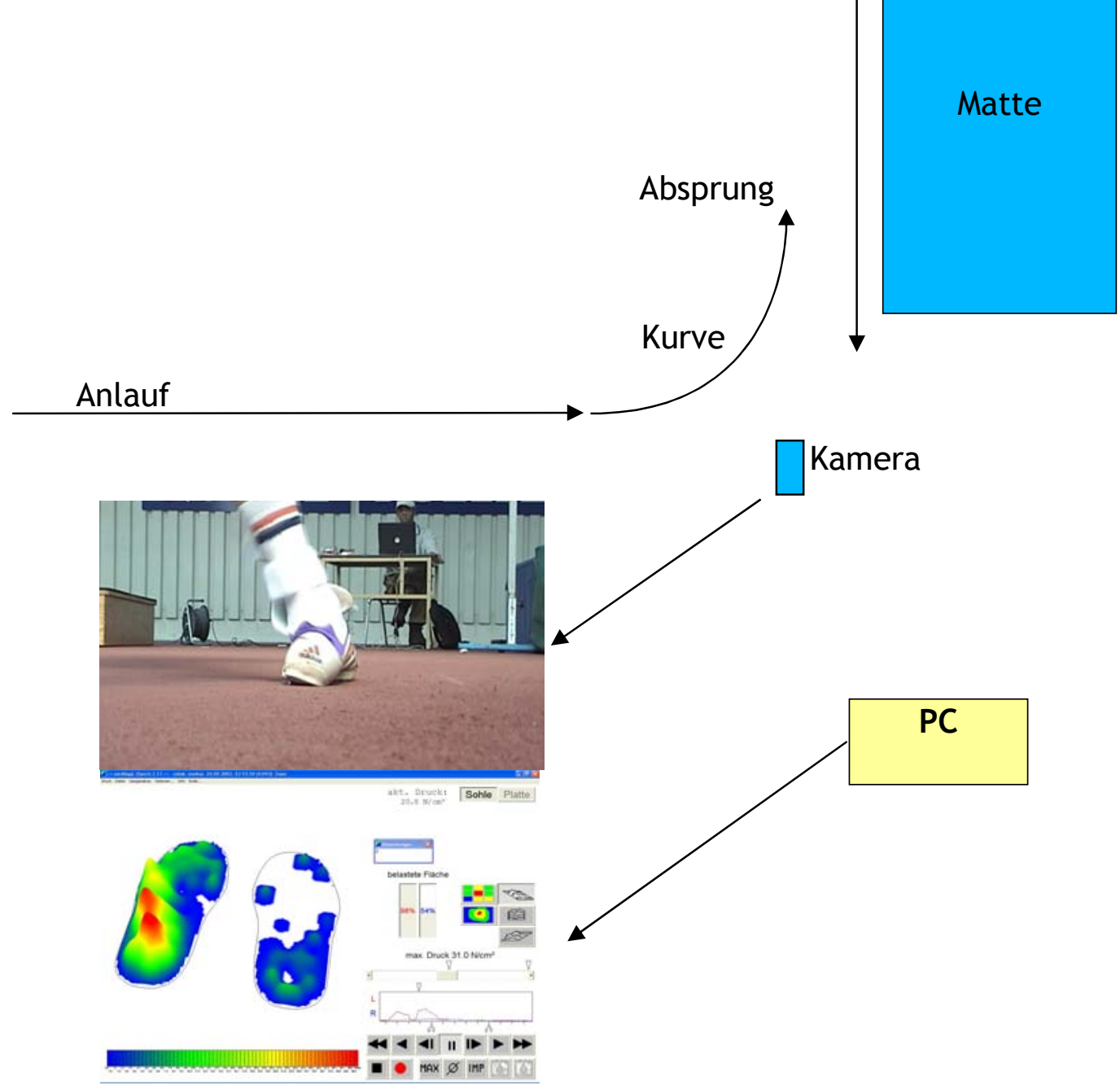


Mittels Wiedergabe der einzelnen Standbilder der Aufzeichnung konnte eine genaue Analyse dieser letzten Phase vorgenommen werden. Im Rahmen der Analysen wurden die Videosequenzen den mittels Messsohlen erfassten Druckprofilen der einzelnen Phasen zugeordnet und in Korrelation gebracht. Der Vergleich der Messreihen sollte untersuchen, ob ein Sprunggelenkstape die Pronationsbewegung während des Absprunges verringert und somit, durch Optimierung der biomechanischen Verhältnisse, eine verbesserte Kraftübertragung und dadurch eine höhere Kraftspitze in den Boden bzw. in die Messsohle bewirkt.

Ferner konnte die Dauer des Bodenkontaktes während des Absprunges gemessen und entsprechend verglichen werden. Das Integral aus der Druckkurve jedes Absprunges ergibt den Impuls und gilt als Maß für die Kraftübertragung. Auch die Ergebnisse dieser Werte haben wir einander gegenüber gestellt.

Vor den Sprüngen mit Tape, sowie nach jedem einzelnen Sprung mit Tape wurde die ROM erfasst und die Zunahme der ROM bzw. die Abnahme der stabilisierenden Wirkung des Tapes gemessen. Die Messtechnik wurde im Abschnitt 4.3.2 'Beweglichkeit' beschrieben. 
4.4 Fragebogen zur Ermittlung der anamnestischen und subjektiven Parameter

Ein Fragebogen, welcher auf die Erfassung der subjektiven Eindrücke und Erfahrungen der einzelnen Athleten abzielte, wurde von jedem der Probanden nach Absolvierung aller Versuchsreihen ausgefüllt. Der Fragebogen umfasste die Parameter:

Generelle Auffassung über den Einsatz von Tapeverbänden Vorverletzung am Sprunggelenk (Sicherheitsgefühl im Sprunggelenk) Vorerfahrung mit Tape Gründe für Nicht-Benutzung von Tape Trainingsumfang in Stunden/Woche Subjektives Gefühl durch Tape Subjektives Gefühl nach Entfernung des Tapeverbandes Zukünftige Favorisierung mit/ohne Tape

siehe ANHANG C 


\section{Ergebnisse}

\subsection{Propriozeption}

\subsubsection{Versuch 1: Dominanz/Dextrosität}

14 von 28 Rechtsfüßern gewichteten ungetaped im Gleichgewichtsstand die rechte Seite stärker als die linke. Mit beidseitigem Sprunggelenkstape fiel in 16 Fällen das Lot rechts der Medianen. Eine 50 \%ige Korrelation zwischen Dominanz und Dextrosität lässt also nicht den Schluss zu, dass Rechtfüßer gleichzeitig rechtsdominant sind.

Insgesamt ist aber in Tape eine Annäherung des KSP an die Mediane zu beobachten. Das deutet auf eine verbesserte propriozeptive Wahrnehmung der Gewichtsverteilung hin.

[Abb. 5.1.1: Überlappend dargestellt sind die Lote der KSP während der subjektiv als gleichmäßig empfundene Verteilung des Körpergewichtes auf beide Beine. In Schwarz die Versuchsreihe ohne Tape, Rot: mit Tape.] 


\subsubsection{Versuch 2: Temperatur}

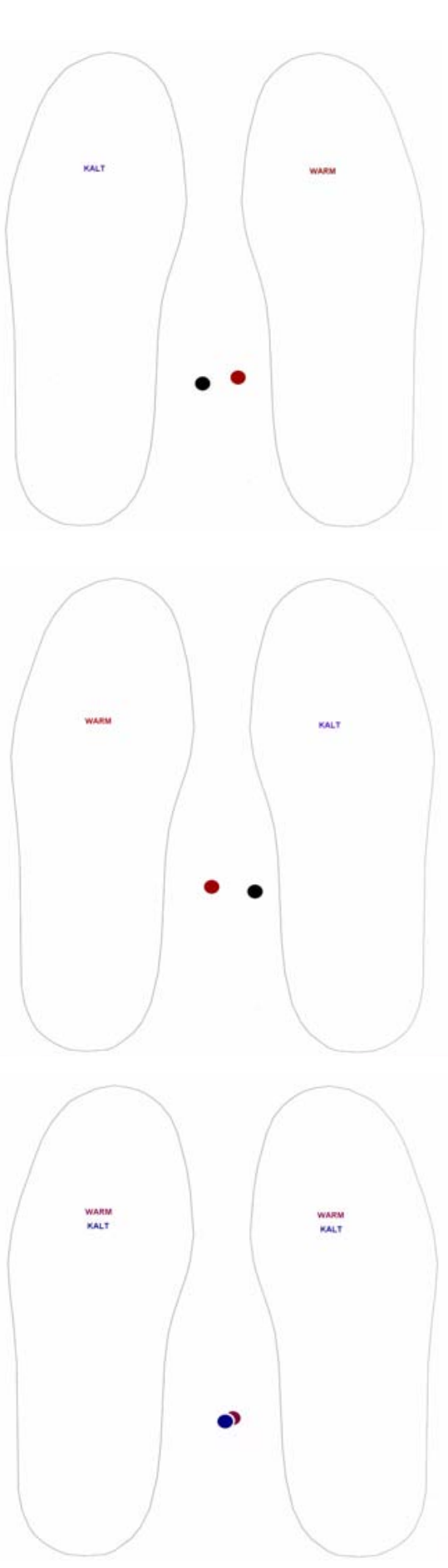

In den Abbildungen stellen die Punkte die

Summe aller gemessenen KSPs dar.

In roter Farbe unmittelbar nach Abkühlung und Erwärmung der Füße. In schwarz die Messergebnisse ohne Erwärmung.

In der Kontrollgruppe (c) stellt der blaue Punkt die Summe aller KSP bei beidseitiger Abkühlung, der rote Punkt bei beidseitiger Erwärmung dar.

Ungeachtet der Dextrosität, konnten wir eine Gewichtung zugunsten des wärmeren, somit propriozeptiv empfindlicheren, Fußes feststellen. (a) und (b)

Die Variante, beide Füße zu erwärmen bzw. beide abzukühlen, erbrachte keine wesentliche Verschiebung des KSP. 


\subsubsection{Versuch 3: Gleichgewicht und Stabilität}

Bei den folgenden Versuchen wurde der Verlauf des KSP-Lotes über dem Standbein aufgezeichnet und mit der Gegenmessung verglichen. Die Abbildungen stellen die Summe aller KSP-Lotverläufe der jeweiligen Messreihe dar. Zur Vereinfachung wurden hier auch die linksdominanten Messungen durch Spiegelung als rechte Sohle projeziert.

Versuch $3 a$ - Einbeinstand
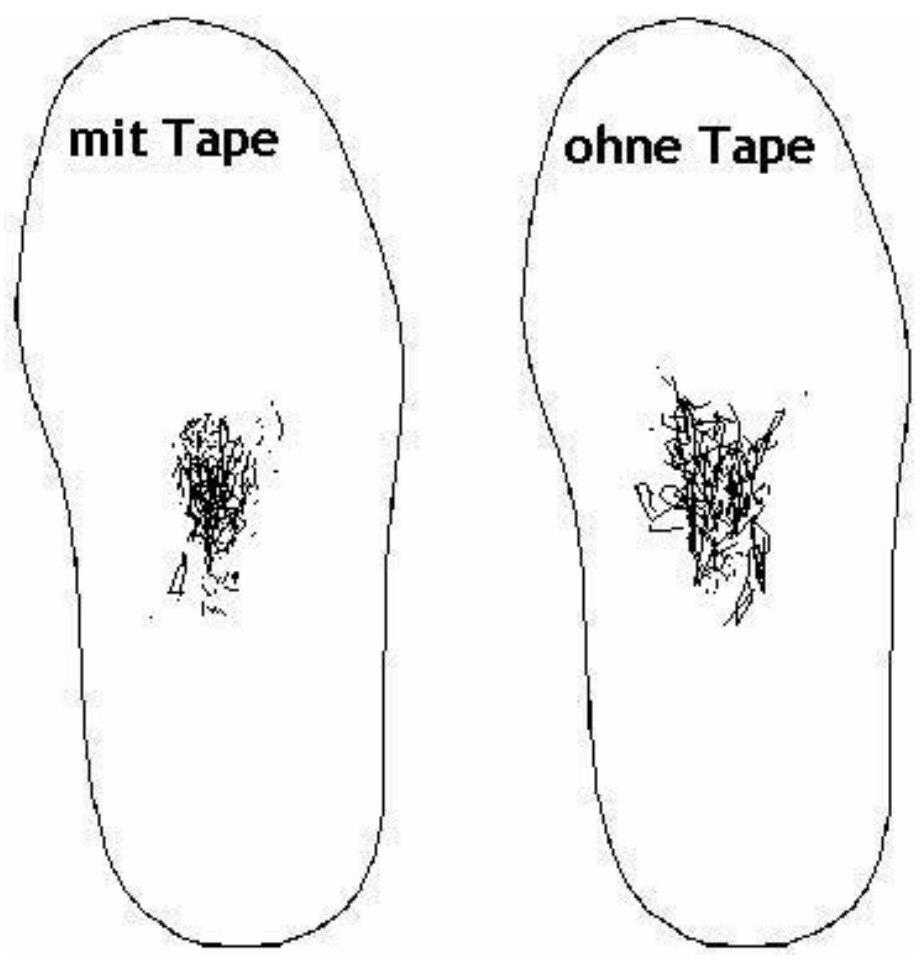

Beim Einbeinstand auf festem ebenen Boden stellten wir eine Tendenz zur kontrollierteren Führung des Gleichgewichtes fest. Wenn auch nur geringfügig, so kann dennoch unter getapeten Bedingungen eine Konzentrierung des KSP über eine kleinere Unterstützungsfläche festgestellt werden. 

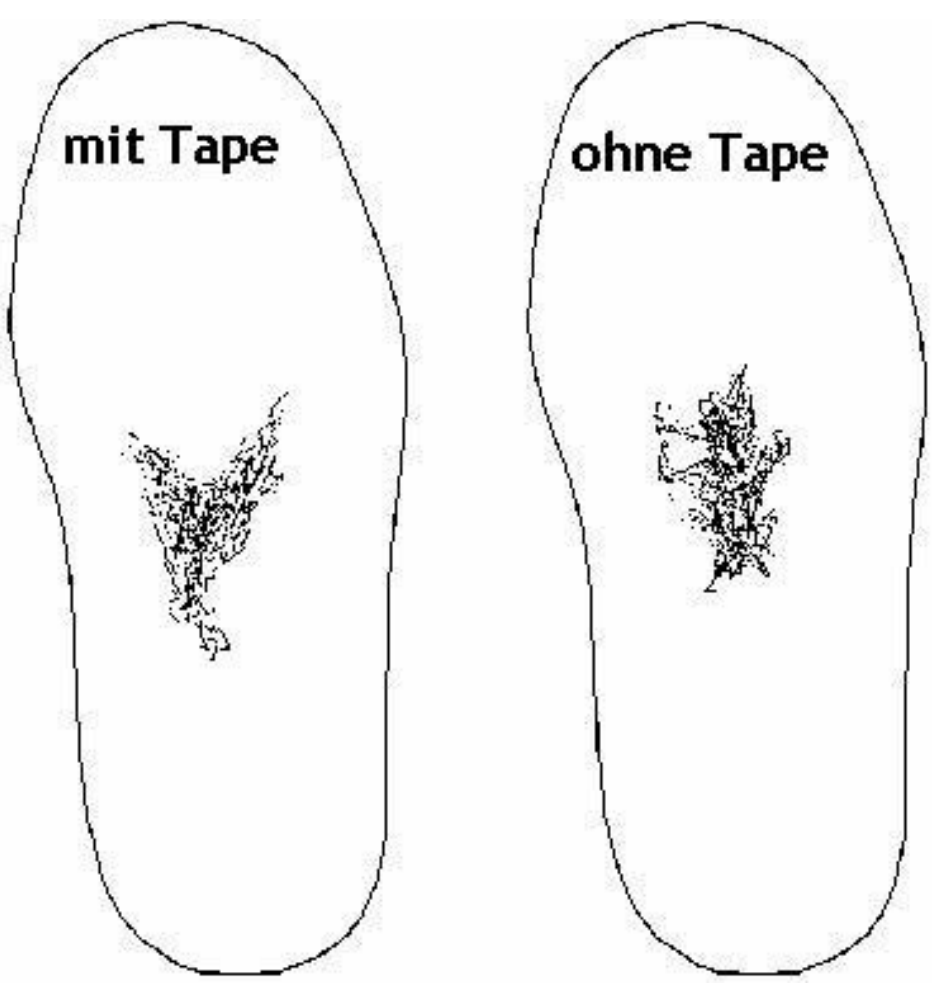

Versuch 3b - Einbeinstand auf POSTUROMED $®$ :

Bei den Messungen während des Einbeinstandes auf der Posturomed $₫$ Plattform stellten wir lediglich einen Unterschied in der Form der Unterstützungsflächen fest. Während die Flächen sich in ihrer Größe kaum unterscheiden, drücken die KSP Verläufe mit Tape Richtung und Lage der physiologisch 3-eckigen Unterstützungsfläche des Fußes wieder (s. Kap. 2.1.2.1 „Statische Biomechanik“). Die Interpretation dieser Beobachtung wäre jedoch hypothetisch und spekulativ. 
Versuch 3c - Einbeinstand auf Therapiekreisel:
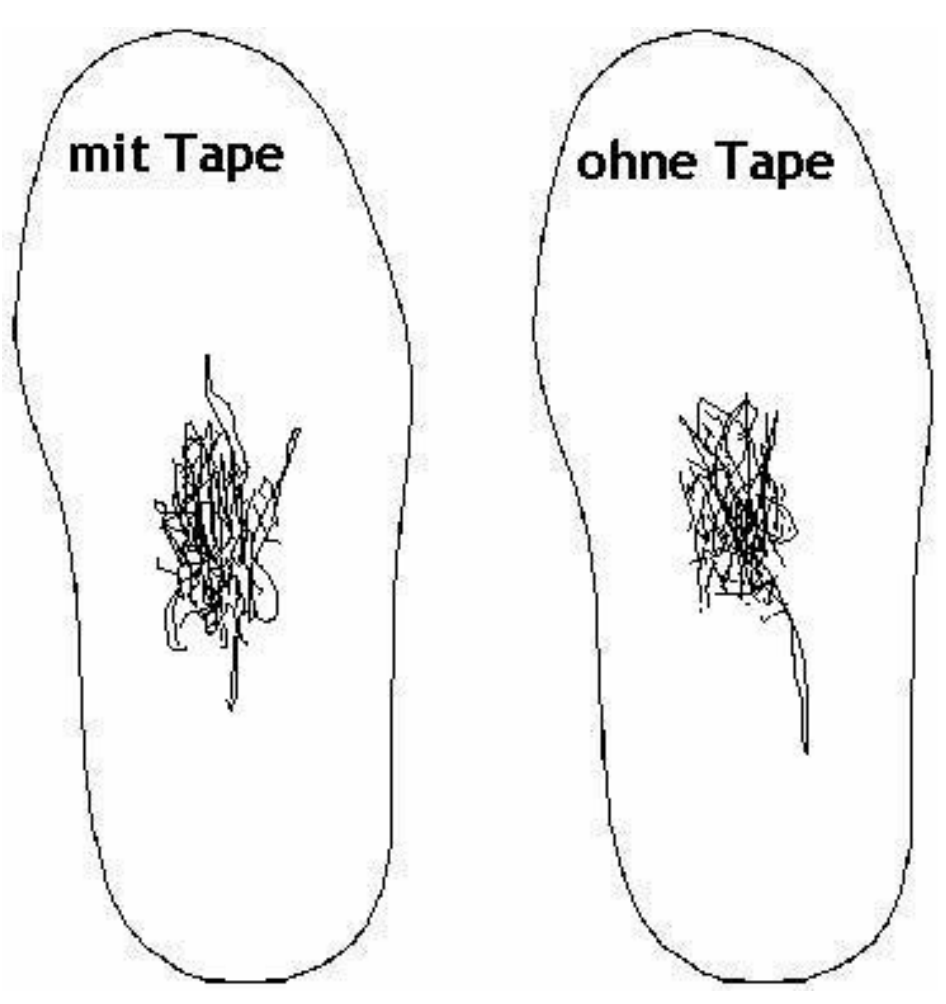

Die Messungen auf dem Therapiekreisel ergaben keine objektiv erfassbaren Unterschiede zwischen mit und ohne Tapeverband. 


\subsection{Leistung}

\subsubsection{Versuch 4: Sprunghöhe}

Die Ergebnisse dieser Versuchsreihe fielen sehr eindeutig aus. Mit Tapeverband war die Sprungleistung aus dem beidbeinigen Stand von und auf den horizontalen Untergrund deutlich eingeschränkt. Die gesprungene Durchschnittshöhe ohne Tapeverband betrug $26 \mathrm{~cm}$, während die mit Tapeverband lediglich $22 \mathrm{~cm}$ betrug. Das entspricht einer Leistungsminderung um $15,4 \%$.

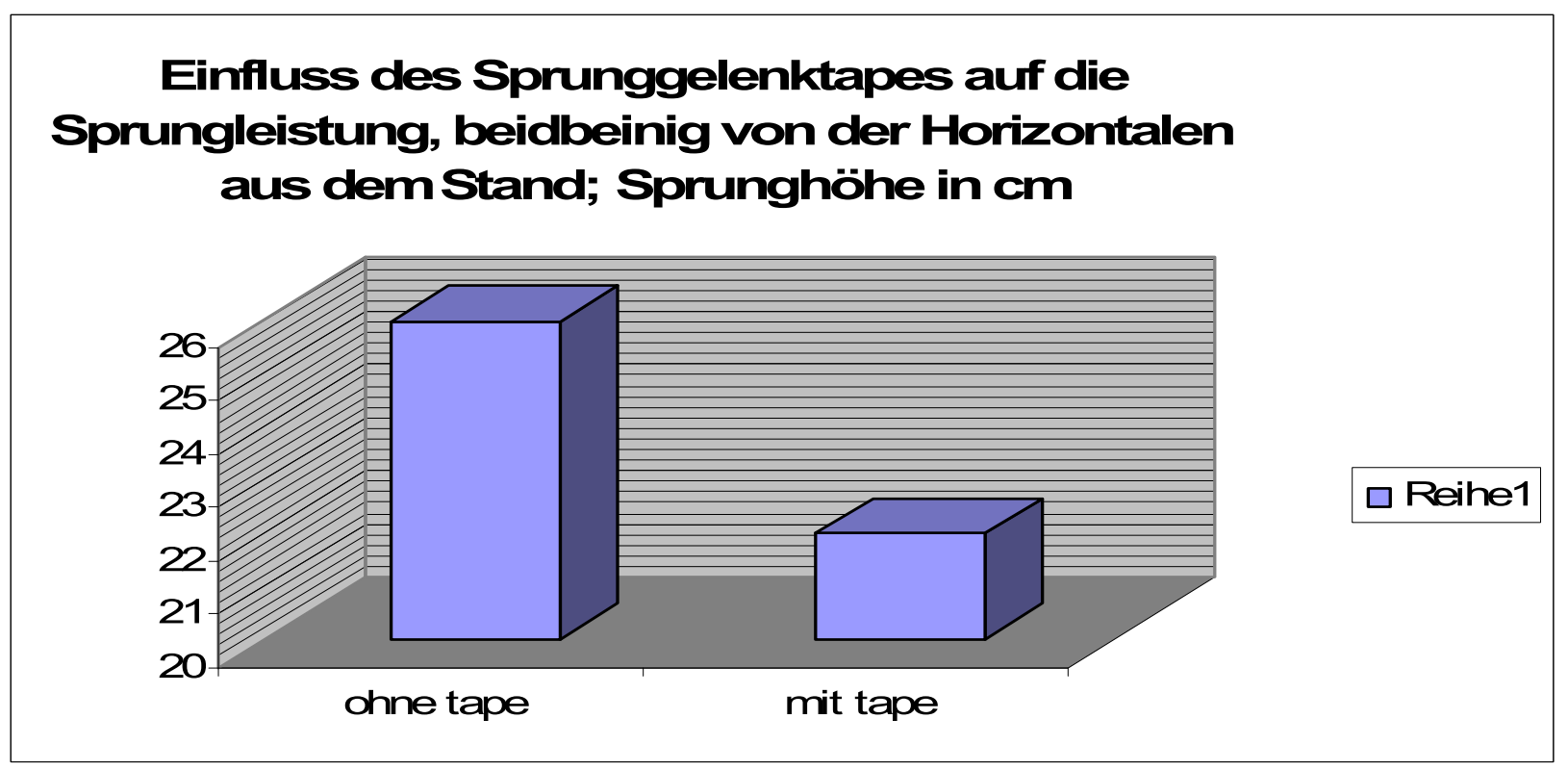

Dieses Ergebnis steht gegensätzlich zu den Resultaten der einbeinigen Sprünge von und auf den mit $35^{\circ}$ seitlich geneigten Untergrund. Zur Erinnerung sei hier nochmals erwähnt, dass jeder Sprung mit einem Doppelschritt als Anlauf eingeleitet wurde. 


\section{max Vertikalsprünge einbeinig nach Arlauf an $35^{\circ}$ Schräge; Sprunghöhe in cm}

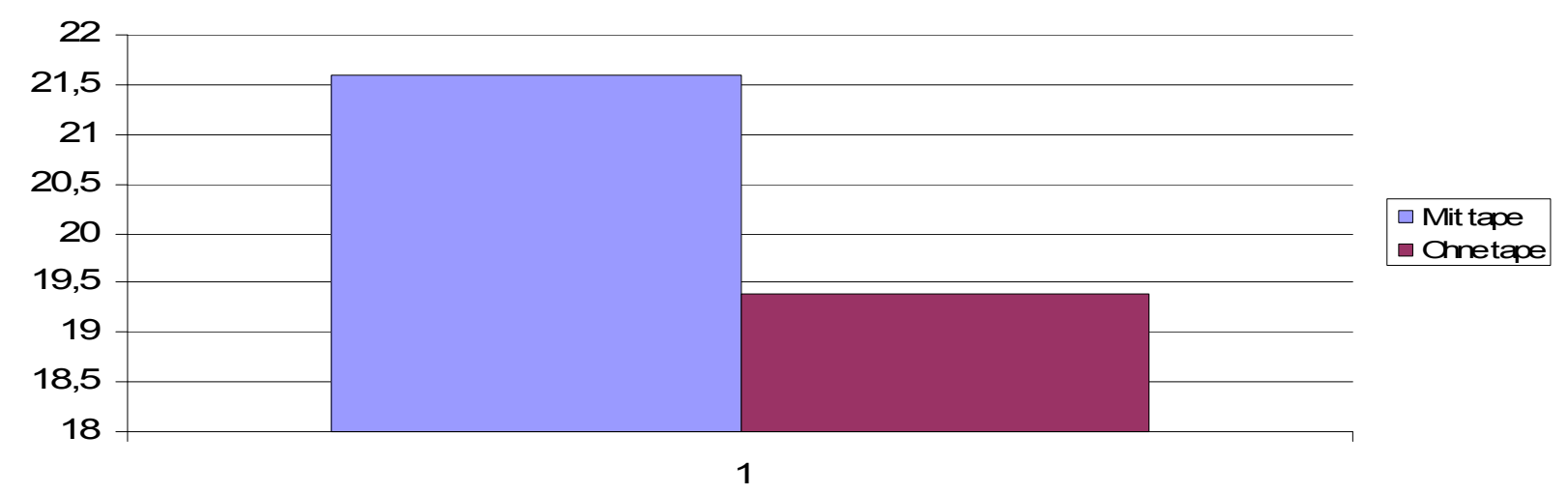

Hier kehrte sich das Leistungsbild um. Die Springer erbrachten unter getapeten Bedingungen mit 21,6 cm eine um 10,2 \% höhere durchschnittliche Sprungleistung als ohne Tapeverband (durchschnittliche Sprunghöhe 19,4cm). 


\subsubsection{Versuch 5: Beweglichkeit (ANHANG E - J)}

5.2.2.1 Sprünge einbeinig am Hang ( $35^{\circ}$ Steigungswinkel)

Der Tapeverband bewirkt eine deutliche Einschränkung der Beweglichkeit, die jedoch schon nach dem zweiten Sprung ein Plateau im Stabilitätsverlust erreicht. Auch nach dem vierten Sprung besteht trotz Bewegungszunahme eine Einschränkung der endständigen ROM.

Flex/Ext vor und nach max. einbeinigen Vertikalsprüngen

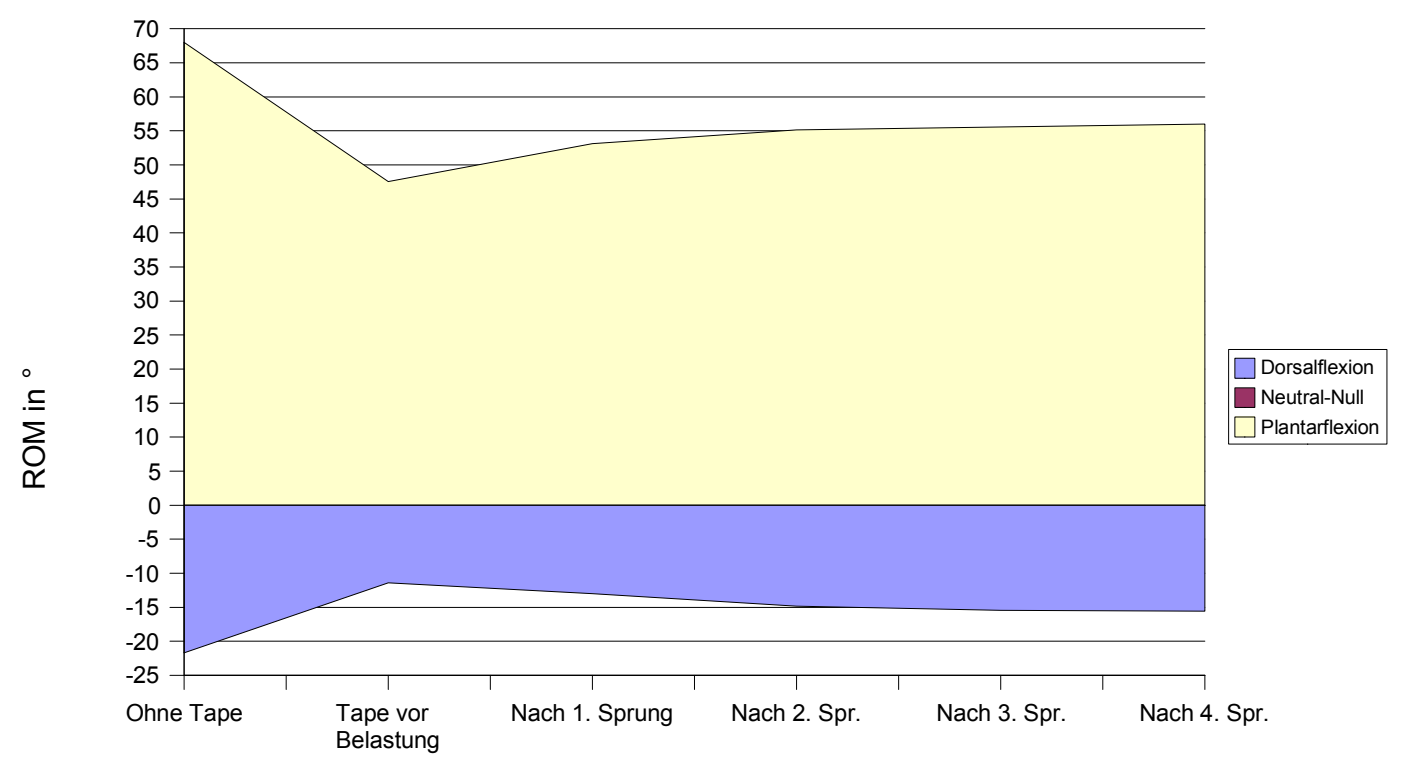


Die angewandte Taping-Technik beabsichtigt einen möglichst geringen Einfluss auf die Flexions-/Extensionsebene, während die Pronations-/Supinationsebene maximal stabilisiert werden soll. Dies wird durch die sehr enge Taille der folgenden Graphik ausgedrückt. Jedoch erreicht der Stabilitätsverlust in der Supinationsebene innerhalb der ersten vier Sprünge kein Plateau, obschon der größte Verlust ebenfalls mit den ersten zwei Sprüngen zu verzeichnen ist.

Supination/Pronation vor und nach max. einbeinigen Vertikalsprüngen

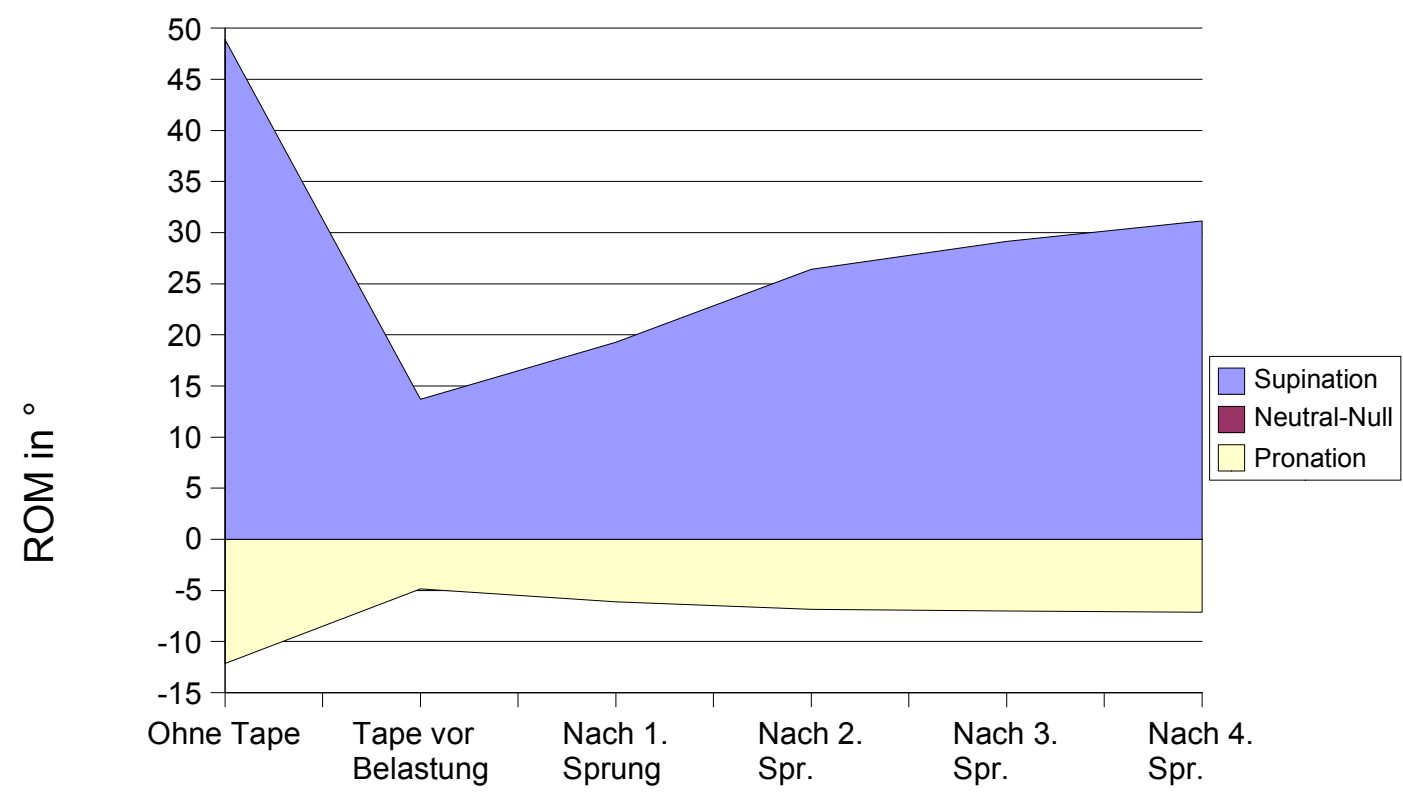

Ebenfalls auffällig ist, dass die passive Beweglichkeit in der PronationsSupinations-Ebene jedoch stärker zunimmt, als bei den beidbeinig durchgeführten Sprüngen. Wir führen dies auf die höhere Belastung durch den schrägen Einsprungwinkel zurück. Ebenfalls kommt es durch die kreisende Kombinationsbewegung während der Abrollphase zu einer Scherbelastung zwischen Tape und Haut. Diese begünstigt die Ablösung des Tapematerials und somit die Abnahme des stabilisierenden Effekts. 
5.2.2.2 Sprünge beidbeinig auf ebenem Untergrund:

Der unter 5.2.1 erfasste Leistungsverlust beim beidbeinigen Sprung mit Tape mag ein Indiz für die eingeschränkte Plantarflexion sein. Das Profil des Stabilitätsverlustes ähnelt dem des einbeinigen Sprunges. Der größte Verlust dieser Stabilität geschieht dann während der ersten 2 - 3 Sprünge und ein Plateau lässt sich erkennen.

Flex/Ext vor und nach max. beidbeinigen Vertikalsprüngen

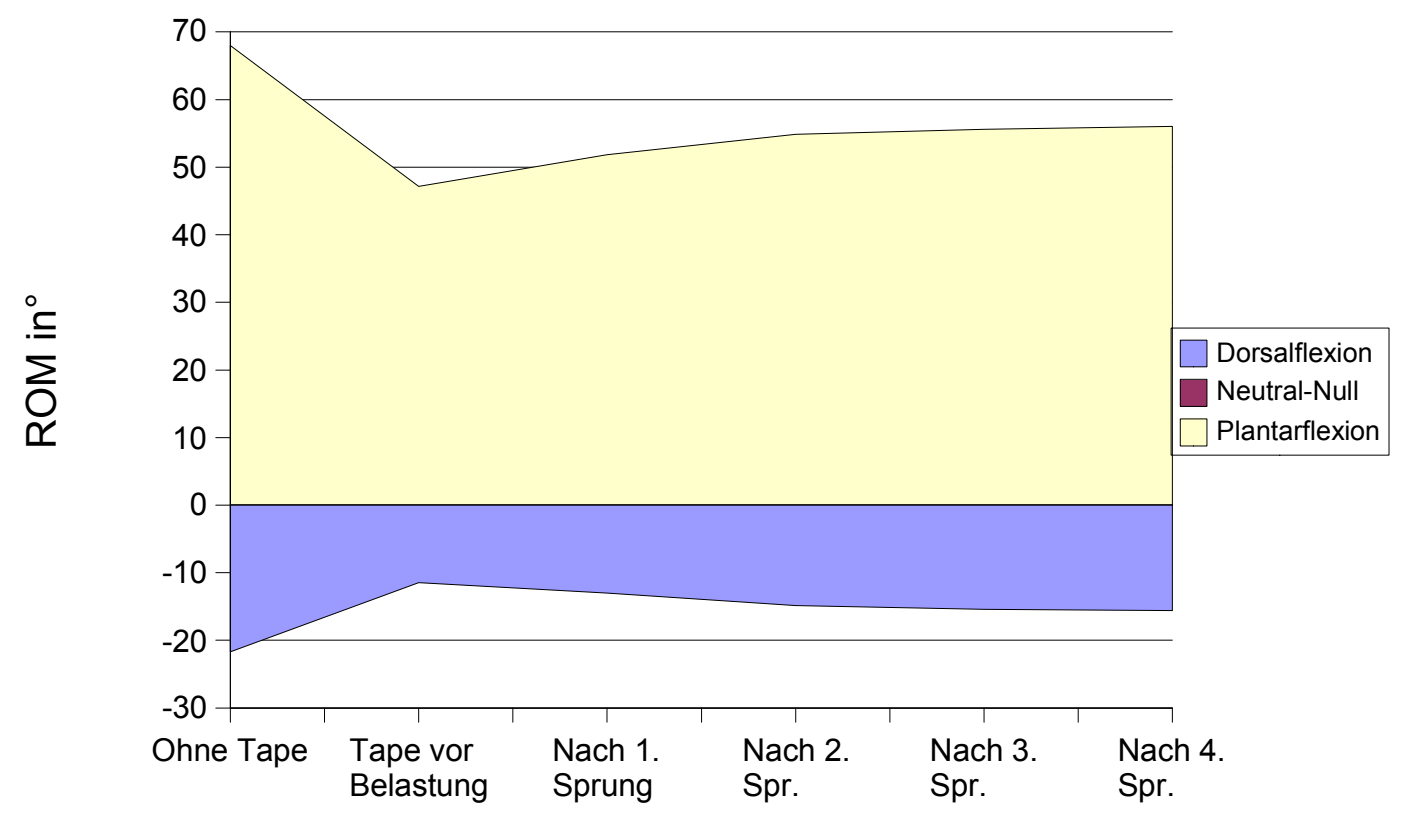




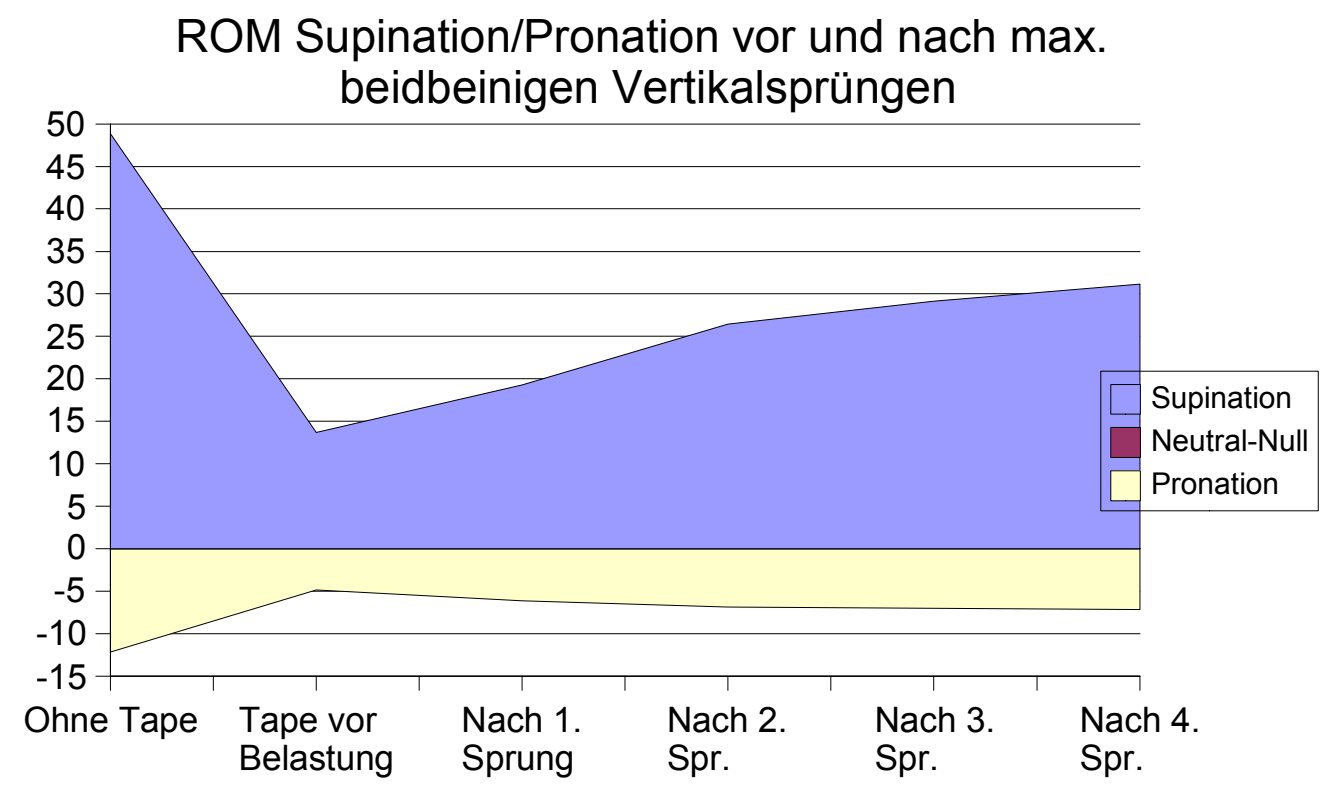

Bekannterweise schränkt Tape die Beweglichkeit in der Supinations-PronationsEbene in höherem Maß ein, als in der Flexions-/Extensionsebene. Auch hier konnten wir einen biphasisch-linearen Einfluss auf die Supination erkennen: Deutlicher Stabilitätsverlust nach zwei Sprüngen, danach beschreibt die Kurve eine flachere Steigung. In der Pronation ist ein geringfügiger stetiger Stabilitätsverlust zu verzeichnen. Dies liegt sicherlich im kleineren physiologischen Aktionsradius, bedingt durch die normale Knochen-BandAnatomie. 


\subsubsection{Einfluss des Hochsprungs auf die Tapestabilität}

Der Einfluss des Hochsprunges auf den Stabilitätsverlust des Tapeverbandes war insgesamt stärker als bei den vorausgegangenen Versuchen. Dies liegt wohl, neben der längeren Belastungsphase durch die zahlreichen Schritte des Anlaufes, besonders an der höheren Belastung, bedingt durch die höhere Geschwindigkeit in den Absprung.

\section{ROM-Sup/Pron vor und nach Taping - Hochsprung}

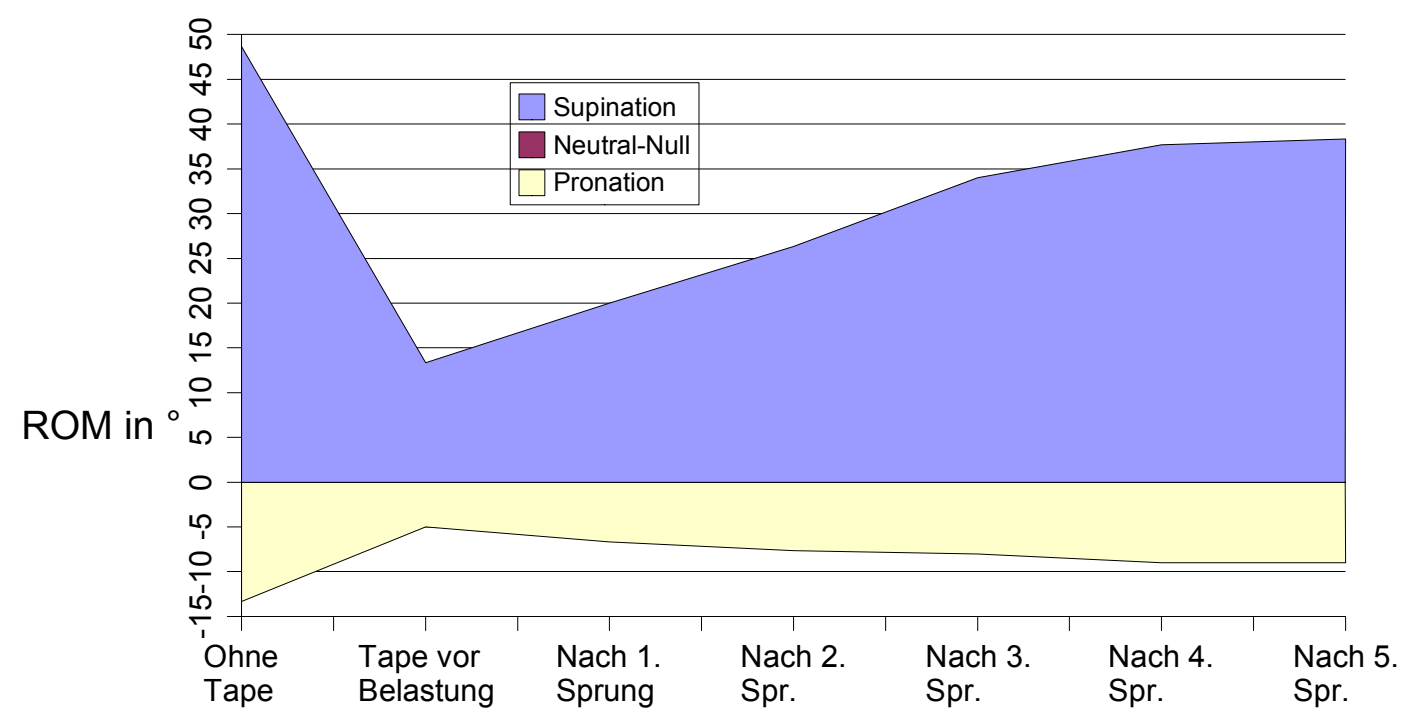

Ein deutlicher Stabilitätsverlust in der Supination, der linear bis zum dritten Sprung eintritt. Erst ab dem vierten Sprung erkennt man einen relativ geringen Einfluss auf eine weitere Lockerung des Verbandes. Die flach auslaufende Steigung im Diagramm ist ein Indiz für den Hauptverlust an Stabilität während der ersten drei Sprünge. Diese Ergebnisse stehen ganz im Einklang mit den Ergebnissen der Dynamikstudien des Absprungs (Abb.: „Wirkung des Tapes auf die Absprungdynamik“). 


\section{ROM-Flex/Ext vor und nach Tape - Hochsprung}

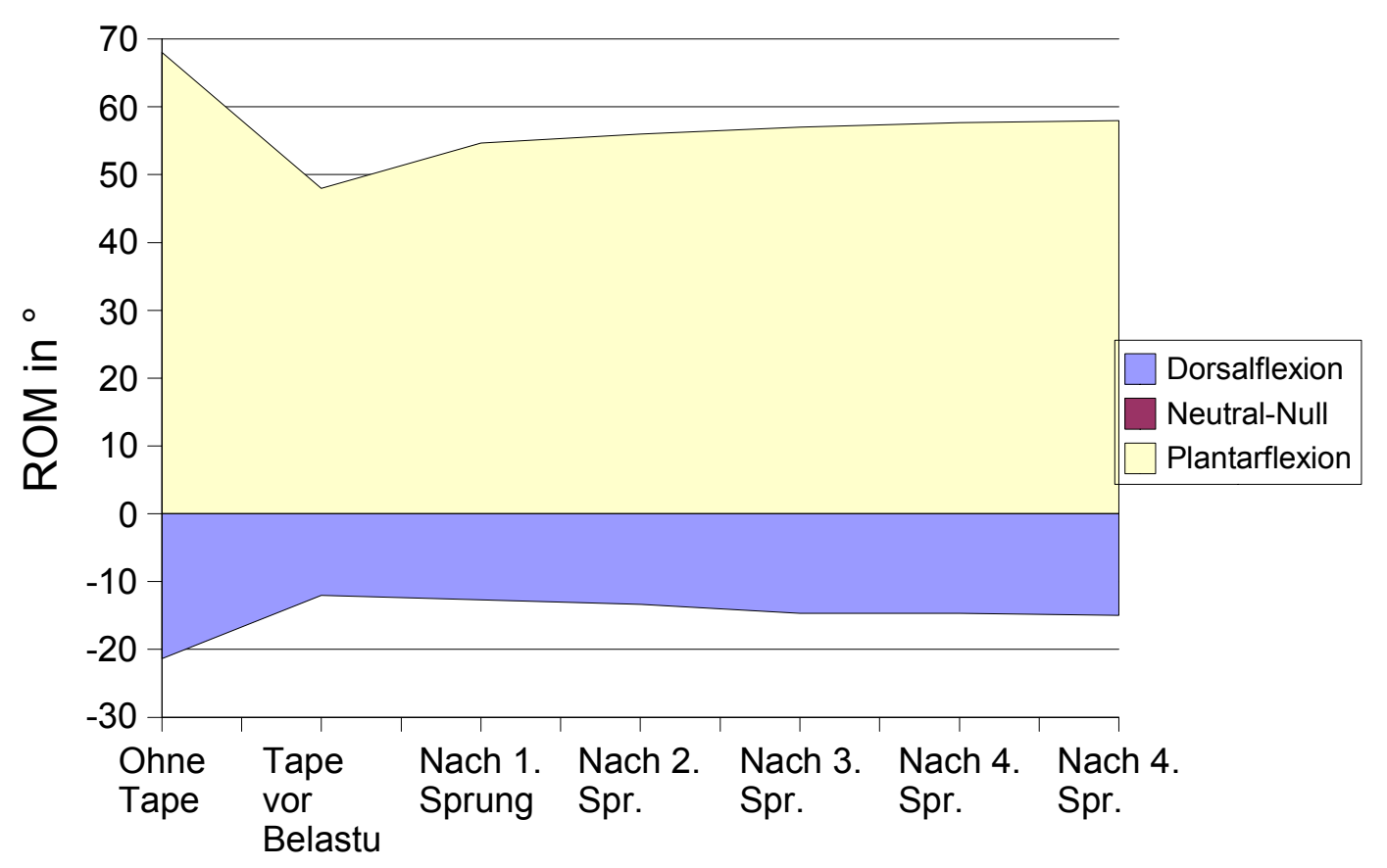

Die Werte in der Flexions/Extensions-Ebene unterscheiden sich kaum in Form und Zahl von den vorausgegangenen Messreihen. 


\subsubsection{Versuch 6: Der Hochsprung}

Weitere Auswertungen des Hochsprungs beschränkten sich auf die Analyse und Interpretation der Druckkurven, die während des Absprunges gemessen worden sind. Wie schon im Vorfeld erläutert, weisen diese ein typisches Druckprofil auf, welches grob in drei Phasen unterteilt werden kann. Es wurden

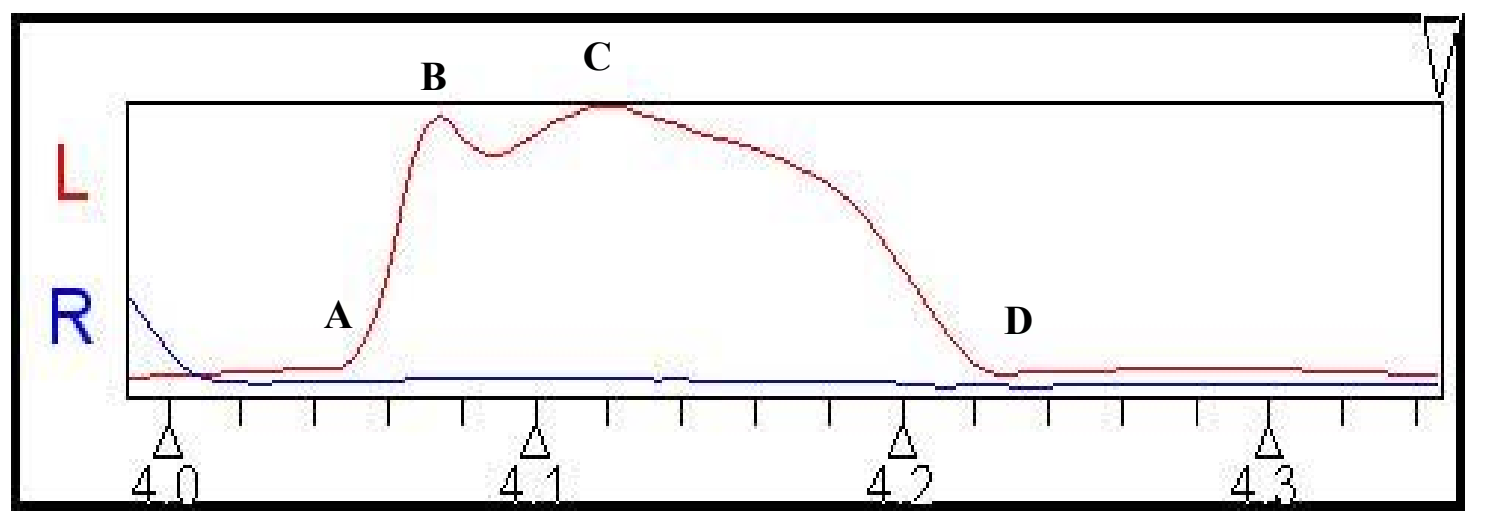

nun die Längen der einzelnen Phasen, sowie die totale Kontaktzeit mit dem Boden errechnet und ausgewertet. Obwohl anzunehmen ist, dass in der Realität die einzelnen Phasen in ihrer Dynamik in einander übergehen und somit nicht scharf gegeneinander abzugrenzen sind, wurden den einzelnen Phasen dennoch definierte Grenzen zugeordnet, nicht zuletzt um die rechnerischen Vergleiche zu erleichtern. Die Stemmphase wurde demnach von der Strecke $A B$ beschrieben, die Standphase wurde von $B$ und $C$ eingegrenzt und der Abdruck drückt sich zwischen $C$ undD aus. 
Druckspitzen während der Stemmphase des Absprunges

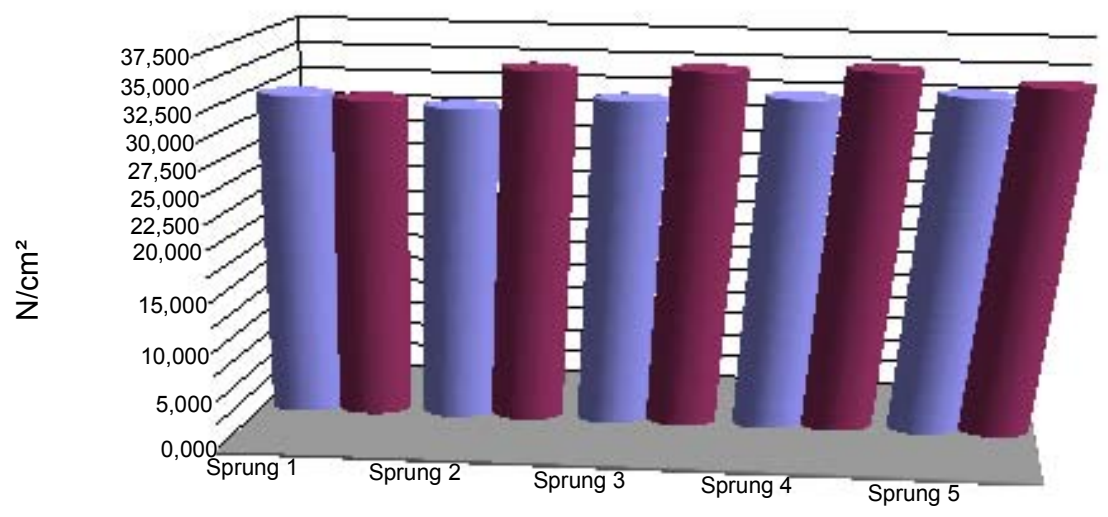

Zunächst werteten wir die Druckspitzen aus, die während des „Einsprunges“ in die Stemmphase des letzten Schrittes gemessen werden konnten. Diese Druckspitzen dienten uns, gemeinsam mit den gemessenen Einsprungswinkeln, als Maß für die Geschwindigkeit zum Zeitpunkt des „Einsprunges“ in diesen letzten Schritt. Bis auf den ersten Sprung, konnten wir im Durchschnitt bei allen Sprüngen einen deutlich höheren Druck unter getapeten Bedingungen feststellen, was wiederum Ausdruck eines größeren Sicherheitsgefühles sein kann. 


\section{Wrkung des Tapes auf die Absprungdynamik}

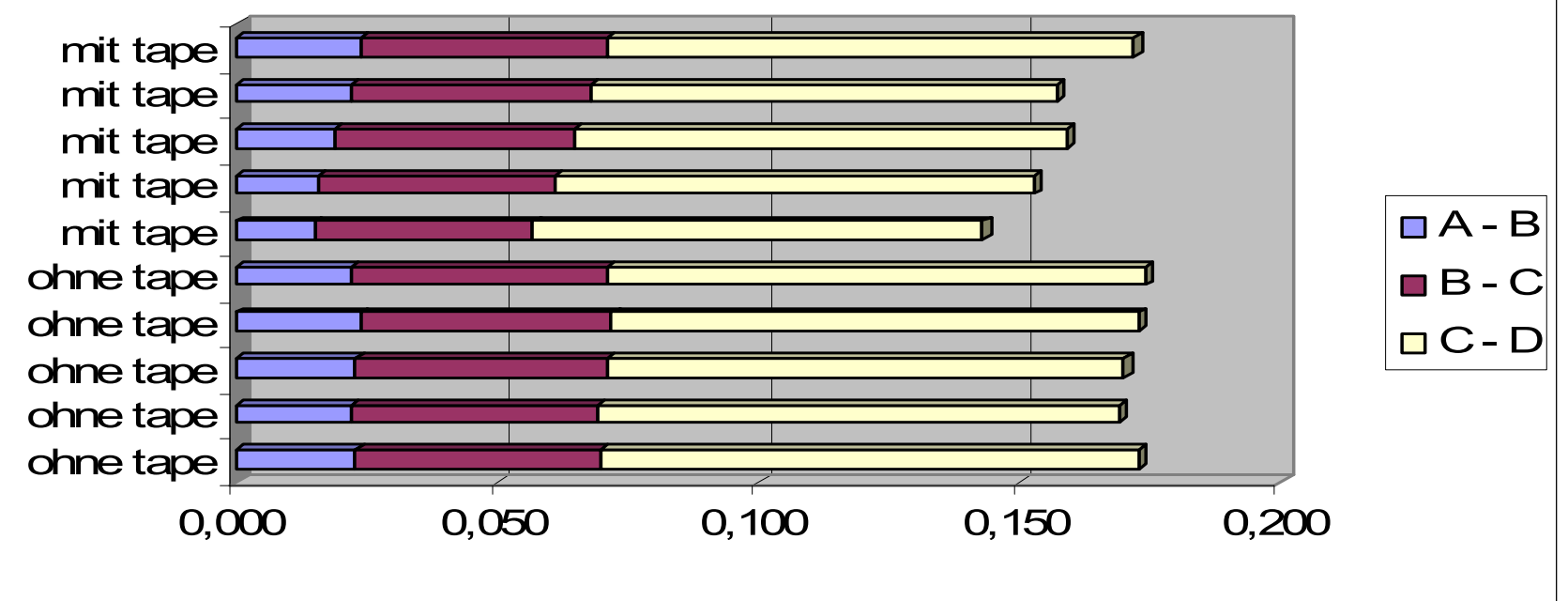

Als nächstes wurden die einzelnen Phasen untereinander verglichen. Auffällig dabei ist die im Durchschnitt signifikant verkürzte totale Bodenkontaktzeit (TBK) mit Tape im Vergleich zu den Sprüngen ohne Tape während der ersten vier Sprünge stattfindet. Mit jedem Sprung verlängert sich die TBK stetig, bis sich ein sehr deutlicher, verlängernder „Sprung“ der TBK nach dem vierten Sprung feststellen lässt. Die Vermutung, dass dieses Phänomen auf die nachlassende stabilisierende Wirkung des Tapeverbandes zurück zu schließen ist, lässt sich durch die nach jedem Sprung zunehmende passive Beweglichkeit bestätigen. Der fünfte Sprung mit Tape gleicht sich in der TBK den Sprüngen ohne Tape an. 
5.3 Fragebogen zur Ermittlung der subjektiven und anamnestischen Parameter - ANHANG A -

Fast alle Befragten (97 - 100\%) waren der Auffassung, dass ein Tapeverband der Rehabilitation und Prävention von Verletzungen vorbehalten sei. 22 (63\%) glaubten, dass ein Tape nur von einem Arzt oder Physiotherapeuten anzulegen sei. Lediglich 8 (23\%) sprachen dem Tape eine leistungssteigernde Funktion zu.

2 (8\%) der Probanden wiesen eine Vorverletzung am Sprunggelenk des Sprungbeines auf.

Während $94 \%$ sich noch nie $(32=91 \%)$, oder nur sporadisch haben tapen lassen, sind nur zwei Athleten dabei, bei denen der Tapeverband regelmäßig zum Wettkampf und/oder Training eingesetzt wird. Der Grund für die hohe Zahl der „Tape-Verweigerer“ ist wohl dadurch bedingt, dass es für diese Sportler nie als Option in Frage gekommen ist. So geben 32 (91\%) an, dass Sie Taping noch nie in Erwägung gezogen haben. Nur einer gab wirtschaftliche Gründe für den Verzicht auf Tape an.

Auf diversen überregionalen Sportfesten mit bis zu 500 aktiven Sportlern, errichteten wir Tapingstationen, welche den Sportlern die Möglichkeit geben sollten, sich kostenfrei tapen zu lassen. Auf diesen Service wurde durch ein Informationsblatt im Begrüßungspaket, sowie durch zahlreiche regelmäßige Lautsprecherdurchsagen aufmerksam gemacht. Dennoch wurden wir lediglich von einer verschwindend geringen Zahl Aktiver aufgesucht, sodass wir zur aktiven Aquise schritten. Dabei stellten wir fest, dass die meisten Sportler gar nicht wussten, was Taping überhaupt bedeutet. Diese Erfahrungen spiegeln einmal mehr den Status des Tapings im deutschen Sport wieder. Während das Taping in den USA mit der Sportausrüstung auf gleicher Stufe geführt und vermarktet wird, ist das Image des Tapes in Deutschland sehr 'medizinisch' und dem allgemeinen Breitensport bislang verwehrt geblieben. 


\section{Diskussion}

Bei der Diskussion über die Rolle des Tapes im Sport lassen sich die Gesichtspunkte Verletzungsprävention, Stabilisierung und Leistungssteigerung nie völlig von einander getrennt besprechen. Insbesondere beim Tape am Sprunggelenk müssen sie aufgrund ihrer gegenseitigen Einflußnahme stets gemeinsam genannt werden. So läßt sich durch eine Stabilisierung durch Tape eine Verletzungsprävention erzielen, die wiederum eine höhere Leistungsfähigkeit bewirken kann.

Die in der Literatur bestehenden Arbeiten lassen insgesamt eine genaue Definition und dadurch Trennung gewisser Perspektiven völlig außer Acht. Beispielsweise ist anzunehmen, dass bei der Wirkung des Tapes auf ein bereits vorverletztes Gelenk eine andere Gewichtung der zugrundeliegenden Mechanismen vorliegt, als im Falle eines gesunden Gelenkes. Selbst unter gesunden Gelenken müsste unterschieden werden zwischen jenen, die an die Grenzen der persönlichen Belastbarkeit gehen und jene, die an die strukturellen Belastbarkeitsgrenzen reichen. Letztere wären Weltklasseathleten, wenn man die aktuell bestehenden Rekorde als Maß für die maximale Leistungsfähigkeit der menschlichen Physiologie und Anatomie ansieht. So muss diesbezüglich die Frage gestellt werden, ob ein Tapeverband beim amtierenden Weltrekordhalter im Hochsprung die gleiche Wirkung, sei es auf die erbringende Leistung oder auf die Stabilität, besitzt, wie beim bayerischen Jugendmeister. Wo wäre der Übergang eines etwaigen Unterschiedes anzusiedeln und wovon wäre dieser Übergang abhängig? 
Unter physikalischen Gesichtspunkten hängt die beim Absprung wirkende Kraft F sowohl von der Gewichtskraft, wie auch von der Beschleunigungskraft ab. Dieser Sachverhalt läßt sich durch die Formel

$$
F=m \times g+m \times a
$$

ausdrücken, wobei $\mathrm{m}$ die Masse, g die Gravitation und a die Beschleunigung ist. Anhand dieser Formel wird leicht ersichtlich, dass die entstehende Belastung auf die Anatomie auch von individuellen Variationen ein und desselben Sprunges abhängig sein kann. Wie im Abschnitt über die Technik des Hochsprunges beschrieben, lassen sich die sogenannten Power-Jumper beobachten, die einen kräftigen Absprung mit dem Kompromiss eines verlängerten Bodenkontaktes ausüben. Diese Technikvariation geht natürlich auf Kosten des im Optimalen möglichst kurzen Bodenkontaktes und führt somit zu einer verminderten Beschleunigungskraft. Es ist anzunehmen, dass auch hier die Wirkung des Tapeverbandes unterschiedlich zu den „perfekten“ Springern ausfällt.

Die physiologische Belastbarkeitsgrenze wird offenbar bei jeder Verletzung überschritten. Dies kann entweder in niederen Leistungsbereichen stattfinden durch schlechte Technik, zu kurze und unangemessene Aufwärmphase, mangelhaftes Material, etc. . Geht man von einer Optimierung aller Faktoren beim Weltklasseathleten aus, bei dem eine ausgereifte Koordination präzise Bewegungsabläufe zuläßt, wird die Leistungsgrenze letztendlich nur von der physiologischen Belastbarkeitsgrenze der anatomischen Strukturen bestimmt. Die Tapeanlage könnte die anatomischen Strukturen unterstützend stärken und somit höhere Belastungen zulassen. Aber geschieht dies auf Kosten der Beweglichkeit? 
In welchem Leistungsbereichen werden die Belastbarkeitsgrenzen des Tapes ausgereizt? Bei U.S. Amerikanischen Footballspielern wird in aller Regel zusätzlich zum Sprunggelenkstape das Sprunggelenk nochmals über dem getragenen Schuh getapet. Man spricht dann vom „Spatting“. Dies soll weitere Stabilität gewähren, um den sehr hohen sportartspezifischen Belastungen Stand zu halten.

Das Prinzip der zirkulären Ummantelung eines Körperteils kennen wir nicht nur im Bereich des Tapings. Gewichtheber aller Leistungsklassen verwenden einen Gurt, den sie sich recht eng um die Taille schnallen. Dadurch wird eine Art Widerlager geschaffen, gegen welchen gepresst werden kann, um möglichst viel des Kraftpotentials entfalten zu können.

Diese Überlegungen zugrunde legend, mag die Frage über Sinn und Zweck des Tapeeinsatzes in unserem Pool erarbeitet sein, dies jedoch nicht für die übrigen Leistungsklassen zutreffen.

Bei der Literaturrecherche war auffällig, dass im Allgemeinen genaue Angaben über Art, Schweregrad und Zeitpunkt der Verletzung gänzlich fehlten. Damit fehlt natürlich auch eine Einteilung und Gegenüberstellung der einzelnen Gruppen. Den Grund hierfür sehen wir, wie weiter unten im Detail erläutert, in der multifaktoriellen und äußerst variablen Natur von Bewegung und Belastung.

Das Sprunggelenkstape wird in der Regel zur Prophylaxe von erstmaligen bzw. wiederholten Verletzungen eingesetzt, welche durch „Umknicken“ (Supination / Pronation) verursacht werden könnten. BALDUINI (1987) und KARLSSON (1989) belegen, dass $10 \%$ bis $20 \%$ aller Supinationstraumen am Sprunggelenk, die mit einer akuten Ruptur das lateralen Seitenbandes einhergehen, in eine 
chronische Sprunggelenksinstabilität münden. Die chirurgische Korrektur chronischer Seitenbandinstabilität liefert laut KARLSSON (1989; et al. 1988, 1989; et Lansinger 1990) in der Mehrheit der Fälle zufriedenstellende Ergebnisse. Dennoch bleibt die Operation eine zeitaufwendige Methode und Sportler ziehen häufig andere Behandlungsalternativen, wie funktionelles Konditionstraining, etc., vor (KARLSSON 1993). Die strukturelle Instabilität ist von der funktionellen Instabilität abzugrenzen. Letztere ist ein komplexes Syndrom, bei der zusätzlich zum strukturellen Kompromiss, auch neuromuskuläre Faktoren zugrunde liegen (KARLSSON 1992).

JEROSCH (1995) verglich Sprunggelenksorthesen und Tape an 16 instabilen Sprunggelenken. Dabei ermittele er im Vorfeld deutliche Unterschiede in der Propriozeption zwischen stabilen und instabilen Sprunggelenken und widersprach damit den gegenläufigen Aussagen von LENTELL (1995). Letztendlich unterstrich ihre Aussage jedoch den fehlenden Einfluss des Tapes auf die Propriozeption, ohne eine zugrundelegende Erklärung. In einer Arbeit 1996, schätzt JEROSCH das verletzungsvorbeugende Potential des Sprunggelenkstapes auf ca. 30 verhinderte Verletzungen pro 1000 ausgesetzte Situationen.

Auch in der Rehabilitationsphase nach Sprunggelenksverletzungen, findet das Sprunggelenkstape seine Anwendung (KRÜGER \& OBERDIECK, 1975), wodurch der Athlet, aus nicht klar definierten Gründen, schneller eine größere Sicherheit in der Durchführung der Rehabilitationsübungen gewinnt. Der genaue Mechanismus hierfür ist ungeklärt, jedoch scheint der Hauptgrund in der verbesserten Propriozeption zu liegen (BENELL 1994; FEUERBACH 1994; HEIT 1996; LEPHART 1997 und NAWOCZENSKI 1985) . Die ersten Veröffentlichungen diesbezüglich von FREEMAN et al. im Jahre 1965, postulieren die passiven Elemente, also den Kapsel-Band-Apparat des Gelenkes, als die Hauptpfeiler für 
die Erhaltung einer funktionellen Stabilität im Sprunggelenk. Diese Theorie wurde von MATTHEWS 1987, RYAN 1994 und ROBBINS et al. 1998 als unzureichende Erklärung verworfen. Ihnen zufolge wird die Wahrnehmung der Gelenkstellung, im Gefüge der ständigen Rückkoppelungsmechanismen, nicht vom passiven Halteapparat (GOODWIN 1972), sondern von der taktilen Wahrnehmung der Fußsohle, sowie von den Muskelrezeptoren vermittelt. Dabei besitzt die Tatsache, ob der Athlet barfüssig ist, keine Relevanz.

In Bezug auf Supinationstraumen des Sprunggelenks versuchten NITZ et. al. 1985 zu belegen, dass auch die neuronale Rückkoppelung nach solchen Verletzungen im lateralen Kompartiment gestört ist. Inwiefern das Tapematerial, welches zumindest teilweise direkt auf die Haut geklebt wird, beispielsweise durch taktile Reize oder durch die gewisse Kompression auf das Muskelgewebe, die aufsteigende Nervenaktivität hemmt bzw. stimuliert, konnte von diversen Autoren nicht genau eruiert werden (KUKULKA et al. 1987 und ROBICHAUD et al. 1996). Dagegen haben ALLISON et al. 1999 die völlige Unabhängigkeit der Nervenleitung vom angelegten Tapeverband belegen können.

Der von LOHRER et al. postulierte propriozeptive Verlust, welcher nach ihren Maßgaben durch Technikmodifikationen, wie z.B. Freilegung der Ferse durch zirkuläres Ausschneiden des Tapes über dem Tuber calcanei, restituiert werden kann, halten wir für obsolet. Unsere Ergebnisse zeigen, dass ein Sprunggelenkstape den propriozeptiven Input verstärkt. Gründe hierfür liegen nicht nur in der mechanischen Koppelung zwischen Fuß und Unterschenkel, wodurch variierende Gelenkstellungen durch Zug an den Hautrezeptoren von Fuß und Unterschenkel vermittelt werden. Es liegt sicherlich auch in der unelastischen zirkulären Dressierung, unter welcher die unterschiedlichen Belastungsdrücke genauer wahrgenommen werden können. Insofern sind wir 
der Meinung, dass eine Freilegung der Ferse keine tragende Rolle in der propriozeptiven Wahrnehmung spielt, zumal die größte Dichte an Propriorezeptoren nicht an der Ferse, sondern im Bereich des Vorfußballens, sowie der Zehenbeeren zu verzeichnen ist.

Der Aspekt der unelastischen zirkulären Dressierung zur verbesserten Belastungswahrnehmung kann wiederum auch für den Gewichthebergurt herangezogen werden. Hierdurch wird ein weiterer Zusammenhang zwischen Gurt, Widerlager und konsekutivem propriozeptivem Gewinn plausibel. Verbesserte Propriozeption, nicht nur in Bezug auf das (Aus-)Maß des Gewichtes, sondern - und wahrscheinlich 'besonders' - auf die Wahrnehmung von Körper- und Gelenkstellungen während der Übung. Gerade beim Gewichtheben hängt die Leistung sehr stark von der Führung des Gesamtmassenschwerpunktes entlang eines idealen motorischen Korridors ab, sodass ein propriozeptiver Gewinn durch den Gurt auch leistungssteigernd wirken könnte. Es bliebe zu erörtern, ob tatsächlich eine subjektive Korrelation hierzu bestünde und wenn ja, ob eine Korrelation zwischen dieser Subjektiven und unterschiedlicher bewältigter Gewichte existiert.

Auch bei der Ausrüstung des Schießsports treffen wir auf Entwicklungen, die die Propriozeption verbessern. So hat sich nicht nur bei der Oberkörperbekleidung eine Jacke bewährt, die stabil, anatomisch geschnitten und relativ rigide ist, sondern auch die Schießschuhe und handschuhe sind hoch geschnitten und umfassen Fuß- und Handgelenk. Besonders effizient trägt der so genannte 'Schießriemen' zur Verstärkung der Propriozeption bei, indem er den Oberarm mit der

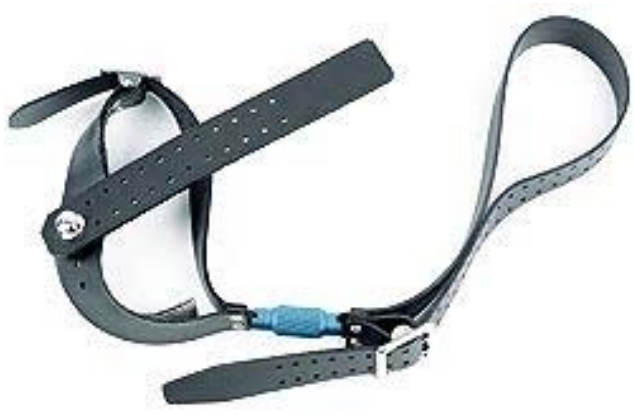


Führhand verbindet und somit kleinste Bewegungen im Schulter-, Ellenbogenund Handgelenk spürbar macht.

Während der Schießsport besonders ausgebildete statische Fertigkeiten abverlangt, bei denen die Beweglichkeit die Leistung eher einschränkt, zählen beim Gewichtheben und noch mehr beim Hochsprung, die dynamischen Komponenten zu den leistungsfördernden Konditionsfaktoren.

Die Problematik im Anlegen eines Tapeverbandes liegt unverkennbar im Kompromiss zwischen der Stabilität und der Beweglichkeit des betroffenen Gelenkes. So würde eine maximale Stabilität die Bewegungsausmaße des Gelenkes stark einschränken und bei unbeeinträchtigter Beweglichkeit wäre die Qualität des Verbandes anzuzweifeln. Der Großteil der in der Literatur vorliegenden Ergebnisse stammen von Untersuchungen über den Einfluss des Tapes auf die Beweglichkeit (ALVES 1992; ANDERSSON 1995; CORDOVA 2000; GARRICK 1973; METCALFE 1997; WILEY 1996; TWEEDY 1994), was in dieser Arbeit zwar mit untersucht wurde, aber eher von zweitrangiger Bedeutung ist. Arbeiten, die wie diese Arbeit, den Effekt des Tapes und der externen Stabilisierung im Allgemeinen, auf die Leistung abhandeln sind rar (MACPHERSON 1995; BRUNS 1992; GROSS 1987, 1991; HUGHES 1983; KARLSSON 1989; SCHEUFFELEN 1993).

CORNWALL (1999) beschreibt mit seinen fokussierten Ganganalysen, dass der Fuß nicht als einzelnes Segment, sondern vielmehr als mehrsegmentales Gefüge funktioniert, in welchem Vor-, Mittel- und Rückfuß gegeneinander beweglich agieren. BRYAN L (2002) stellten Messungen an, die eine Verkürzung der Zeitspanne bis zum Erreichen des Spitzendrucks bei Landungen belegen. Diese Ergebnisse konnten wir mit unseren Versuchen ebenfalls bestätigen. Als Grund 
für diese Beobachtung kann eine Versteifung des Fußes durch die Tapeanlage angeführt werden.

Die flexible Beweglichkeit zwischen den Segmenten bedeutet eine gewisse Elastizität, welche wiederum in Form einer Energiekonservierung zu höheren Sprüngen verhelfen müsste. Die Resultate, die wir mit maximalen beidbeinigen Vertikalsprüngen erhielten, beweisen das Gegenteil. Mit Tape sind alle Probanden niedriger gesprungen, als ohne Tape. Hingegen erhielten wir gegenteilige Ergebnisse im Rahmen der einbeinigen Vertikalsprünge an der Schräge. Wir postulieren hier den stärker Einzug gewinnenden Faktor des Sicherheitsgefühls, der den Absprungsdruck (Impuls, Beschleunigung) an der Schrägen dem unter kontrollierten Bedingungen beidbeinig ausgeführten Sprung von der horizontalen Fläche am nächsten kommen lässt.

Regelmäßiger Sport bietet im Allgemeinen die sozialwirtschaftlich günstigste und nachhaltigste Methode zur Prävention von Erkrankungen, trotz des relativ hohen Anteils der Sportverletzungen an den Gesamtverletzungen (CHAN 1993). Daher ist die Diskussion um den Einsatz von externen Stabilisatoren zur Verletzungsprävention sehr berechtigt.

Die Meinungen über die Funktion des Tapes als Stabilisator sind sehr gegensätzlich. Viele Autoren wollen belegen (MALINA 1962, RARICK 1962, ALVES 1992, FUMICH 1981, FRANKENY 1993, STUESSI 1987, HAMILL 1988, MARTIN 1993, BARTOLD 1992, HUGHES 1983, GROSS 1991, GREENE 1990, GROSS 1994, WILEY 1996, LAUGHMAN 1982, MYBURGH 1984, WILKERSON 1991, SPRINGINGS 1981, HOCHMANN 1982, VAES 1998, ANDERSON 1995, MONEY 1994, GLICK 1976, BAUER 1988, SCOTT 1994, METCALFE 1997), dass Tapeverbände 
bzw. äußere Bandagen ihren Effekt nach Belastung unterschiedlicher Dauer und Intensität verlieren bzw. keinen stabilisierenden Vorteil gegenüber der Kontrolle ohne Bandage besitzen. Die Quintessenz fast aller dieser Arbeiten ist, dass der Tapeverband letztendlich keine vorbeugende Maßnahme hinsichtlich einer Gelenkverletzung darstellt.

Trotz der limitierten klinischen Studien in Bezug auf den verletzungspräventiven Effekt von sprunggelenksstabilisierenden Tapeverbänden, äußerte SITLER 1992 die Vermutung, dass Tape durchaus einen signifikant präventiven Einfluss habe.

Eine sehr häufig zitierte prospektive, randomisierte Studie, welche an 2562 USCollege-Basketballspielern von Garrick JD et al. (1973) ausgewertet wurde, möchte belegen, dass die Spieler mit Tapeverband deutlich seltener Verletzungen erleiden, als jene ohne Tapeverband. Zu kritisieren ist bei dieser Arbeit

Tape als Verletzungsprophylaxe

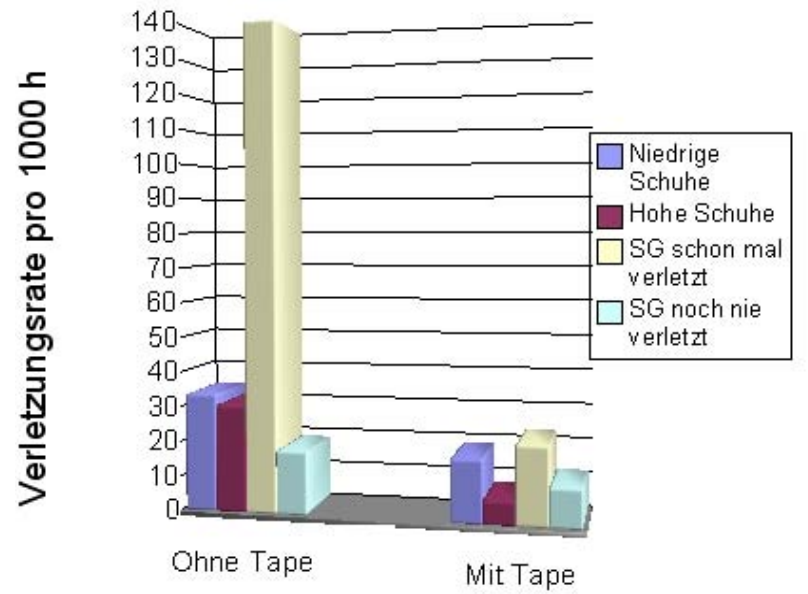
das Fehlen der genauen Diagnosen. Es werden keinerlei Angaben über Art und Schweregrad der Verletzung gemacht. Ferner, wird keine Unterscheidung zwischen Spielstärke, Fitness, Position und bis zum Eintreten der Verletzung am Stück abgeleistete Spielzeit des einzelnen Athleten getroffen. 
Wir teilen die Auffassung mit HUME et al. (1998), dass eine detaillierte Kategorisierung in der Studie von GARRICK durchaus eine höhere Aussagekraft erbracht hätte. Wenn man seine Zahlen dennoch als Maß für die Stabilisationsfähigkeit nimmt, so bietet ein Tapeverband auch nach längerer Belastung eine gewisse Festigkeit. Der Grund für diese Diskrepanz mag in darin liegen, dass die von den besagten Autoren beschriebenen Untersuchungen hinsichtlich der Stabilität, alle den Beweglichkeitsverlust bzw. -gewinn vor bzw. nach der jeweiligen Belastungsperiode gemessen haben. Da Gelenkverletzungen jedoch in den aller seltensten Fällen im Rahmen der physiologischen Beweglichkeit eines Gelenkes statt finden, verliert der Begriff der Stabilisation somit durch die sehr vage Definitionsfähigkeit an Argumentationskraft.

Neurophysiologische Tests sowie Untersuchungen der Wahrnehmung von McCLOSKEY et al. (1977) haben gezeigt, dass die kinästhetische Wahrnehmung, also das Bewusstsein über die Fußposition, fast ausschliesslich von Muskel- und taktilen Hautrezeptoren vermittelt wird. Damit wurden ältere Auffassungen, beispielsweise von BROWNE (1954) und CLARK (1986) verworfen, welche annahmen, dass das Bewusstsein über die Gelenkstellung auch via Rezeptoren in der Gelenkkapsel, sowie durch undefinierte Rezeptoren auf der Gelenkoberfläche und in den Ligamenten, sowie über Rezeptoren in den Sehnen gesteuert wird.

CLARK (1986) widerlegt in seiner Arbeit schon die vermeintliche Signifikanz der Rezeptoren auf der Gelenkoberfläche und in der Gelenkkapsel. In seinen Untersuchungen findet er keinen signifikanten Verlust des Bewusstseins über 
Gelenkstellung nach Gelenkersatzoperationen, bei welchen nicht nur die Gelenkoberfläche ersetzt wird, sodern auch die Kapsel mehr oder minder zerstört wird.

Schon 1972 fanden GOODWIN et al. durch lokale Betäubung des Kapsel-BandApparates heraus, dass Ligamente keinerlei Einfluss auf die Wahrnehmung über die Gelenkstellung nehmen. Außerdem konnten sie nachweisen, dass es trotz veränderter Gelenkstellungen zu keiner signifikanten Änderung der Impulsaktivität von den Sehnendehnungsrezeptoren kommt, sodass dies ebenfalls nicht als zuverlässige Erklärung akzeptiert werden kann.

ROBBINS et al. beschreiben in ihrer Arbeit 1995 einen Versuchsaufbau, in dem di®Probanden mit je einem Fuß auf einen Block steigen. Die Oberfläche einer der Blöcke wird in variablem Ausmaß und Richtung geneigt. Die Probanden sind mit einer Augenklappe versehen, um den optischen Feedback auszuschließen. Nun geben die Probanden, mit Tapeverband bzw. ohne Tapeverband, die wahrgenommene Diskrepanz in der Steigung der geneigten Unterlage an. Die Versuche vor und nach einer 30 minutigen Spiel- und Laufperiode (Basketball) durchgeführt und einer Kontrollgruppe ohne Spiel- und Laufperiode gegenübergestellt. Alle Testreihen wurden jeweils einmal mit und einmal ohne Schuhwerk durchgeführt. Als Resultat kam heraus, dass der Tapeverband als Wahrnehmungshilfe erst ab einem Oberflächenwinkel von $10^{\circ}$ und darüber einen relevanten Einfluss auf die Kinästhesie nimmt. Darüber hinaus geht aus ROBBINS Studie hervor, dass die Fehlerquote bei den Messungen mit Schuhwerk und ohne Tapeverband am höchsten ist, gefolgt von der Konstellation Schuhwerk mit Tapeverband. Am sensibelsten reagieren wir demnach ohne Schuhwerk und mit Tapeverband. 
Dieses, die Ergebnisse von GARRICK und die Tatsache, dass in Populationen, die barfüßig ihren Alltag bestreiten, Sprunggelenksverletzungen extrem selten sind (ROBBINS 1995), legen die Vermutung nahe, dass das Schuhwerk, durch Desensibilisierung der Kinästhesie, das Verletzungsrisiko erhöht. Es wäre wissenswert zu untersuchen, inwiefern dies auf die in der Leichtathletik bzw. im Hochsprung benutzten Schuhe zutrifft. Diesen Punkt aufnehmend, liegt es unserer Meinung ebenfalls nahe, dass durch Schuhwerk sehr ungünstige Hebelbedingungen geschaffen werden. Im Stand verleiht der Schuh, durch die in der Regel flache Sohle eine stabile, aber schmale Unterstützungsfläche. Fällt das Schwerpunktslot mal außerhalb dieser Fläche, wie zum Beispiel bei Sportarten, die häufige Richtungswechsel vorsehen oder auch bei Kontaktsportarten, wird ein „Point of no return“ überschritten und das Gelenk wird in unphysiologische Bewegungsausmaße gezwungen. Der äußere Bandapparat des Sprunggelenks ist bei allen sportlich aktiven Menschen am häufigsten sportbedingt verletzt (RYAN 1986, CHAN 1993, SCHAFLE1993, HOLMER 1994, SHAWDON 1994, BLADIN 1995).

Barfüßig bietet die äußere Anatomie der Ferse eine runde Abrollfläche auch seitlich an. Es liegt eher ein dem indifferenten Gleichgewicht nahe kommender Zustand vor, bei dem keine Kippbewegungen zustande kommen. Dies läßt sich leicht nachvollziehen, wenn man aus dem Stand heraus die Außenknöchel nach außen hin dem Boden nähert - einmal mit Schuhen und dann barfüßig. Dieser Sachverhalt unterstützt die oben erwähnten Beobachtungen von ROBBINS (1995). Sprunggelenksverletzungen geschehen vornehmlich beim Laufen oder Springen während der Absorptions- und Amortisationsphase (SUBOTNICK 1989). Dabei wird das Gelenk in der Regel in die Supination-Plantarflexion gedrängt (MCCONKEY 1987). 
Die Schuhindustrie versucht mehr Stabilität zu erzielen, indem zusätzlich zum Entwurf von hohen Schuhen auch seitliche Stabilisatoren in die Schuhverkleidung integriert werden. Die Studie von ROBBINSON (1986) zeigt, dass die hierdurch geschaffene Stabilität zu einer deutlichen Minderung der Beweglichkeit führt und sich dies in einer Leistungsminderung ausdrückt. In seiner Studie hat er seine Vermutungen anhand vergleichender Läufe in einem Hindernissparcour quantifizieren können. Die Tatsache, dass die Tests lediglich an sechs Individuen ( $n=6$ ) durchgeführt wurden (drei davon vorverletzt), ist unerer Meinung nach kritisch zu bewerten.

Ein großer Nachteil des Tapeverbandes ist seine einwegige Nutzung. Nach Entfernen eines Tapes muss ein neuer Tapeverband angelegt werden. Dies wirft, insbesondere seit dem Erscheinen von immer leichter, handlicher und stabiler konstruierten Orthesen (starr oder flexibel) auf dem Markt, die KostenNutzen-Frage des Tapeverbandes auf. Viele Arbeiten behandeln daher den Vergleich zwischen Tape und Orthese hinsichtlich Stabilität, Verletzungsprophylaxe und des Einflusses auf die Leistung. Im Folgenden werden nun alle von uns recherchierten Arbeiten mit Versuchsaufbau und Ergebnissen im Einzelnen vorgestellt und besprochen.

In METCALFEs (1997) Versuchsaufbau führten 10 gesunde College Studentinnen Vertikalsprünge und einen Leistungstest (Southeast Missouri agility test) durch. Die Versuchsreihen wurden jeweils mit (a)Tape (Steigbügelzügelung im Korbflechtmuster), (b) mit Tape (wie in (a)) zusätzlich verstärkt mit extra Steigbügelzügelung, (c) mit SwedeO- Universal TM (sockenähnlicher mit Schnürsenkel versehener Stabilisator des Sprunggelenks) und (d) ohne externem Stabilisator zur Kontrolle. Die Tests wurden an zwei Tagen durchgeführt, wobei jedoch nicht klar wird, ob und wie viel Tage zwischen den Testtagen lagen. Die Ergebnisse zeigen eine deutlich verringerte Leistung unter externer 
Stabilisierung im Vergleich zur Kontrolle ohne Unterstützung. Dabei ergaben die Ergebnisse der drei verschiedenen Stabilisierungen keinen signifikanten Unterschied zueinander.

HOCHMAN et al. (1982) kamen zu einem ähnlichen Schluss. Dabei wurden der Tapeverband mit einem Stabilisator andere Marke (MIKROS TM) verglichen. Neben Vertikalsprüngen, führten sie ebenfalls beidbeinige Weitsprünge durch, allerdings nachdem ihre Probanden mit Tape eine 90-minutige sportliche Belastung absolviert hatten. Die Ergebnisse erbrachten auch hier keinen wesentlichen Unterschied zwischen den zwei Stabilisationstechniken, sehr wohl aber im Vergleich zur ungestützten Kontrollreihe. Unserer Meinung nach bietet eine „90-minutige Belastungsphase“ zu viele unkontrollierbare Faktoren als Fehlervariable auf. Dementsprechend fällt der Zustand und damit der restriktive Charakter des Tapeverbandes entsprechend der individuellen Fertigund Fähigkeiten des Einzelnen unterschiedlich aus. Zusätzlich unterliegt der beidbeinige Weitsprung vielen individuellen Faktoren, im Rahmen der intraund intermuskulären Koordination, welches auch die Aussagekraft der Messungen relativiert.

PARIS (1992) untersuchte bei 18 Elite-Fußballspielern vergleichend den Einfluss von Tape und verschiedenen Sportorthesen des Sprunggelenks auf Laufschnelligkeit, Gleichgewicht, Beweglichkeit und vertikale Sprunghöhe. Als Kontrollreihe diente die Testreihe ohne externe Unterstützung. Dabei kam lediglich eine signifikante Verminderung der vertikalen Sprunghöhe mit einer der Sportorthesen (New Cross ${ }^{T M}$ ) heraus. Die vergleiche sämtlicher anderer Testreihen ergaben keine signifikanten Unterschiede. 
Einen ähnlichen Versuchsaufbau beschreibt BURKS (1991) an 30 Collegeathleten ohne Spezifizierung hinsichtlich der Disziplin, Spielposition und Spielstärke. Wie bei PARIS 1995 führt er Versuchsreihen jeweils ohne äußere Stützung, mit Tape und mit zwei unterschiedlichen Sportorthesen durch. Die Testreihen beinhalteten den beidbeinigen Weitsprung, den beidbeinigen Vertikalsprung, einen Hin und Rücklauf entlang einer 9 Meter (10 Yards) langen Strecke, sowie einen Sprint über 36 Meter (40 Yards). Aus unserer Sicht bieten auch diese Versuchsreihen keinen echten Boden für Resultate, die eine Quantifizierung der Leistung zuließen. Test wie von BURKS durchgeführt beinhalten zu viele Variablen und sind deshalb zu störanfällig.

In seiner Arbeit vertritt BURKS (1991) im Konsens mit RARICK (1962) und ALVES (1992) die Meinung, dass hauptsächlich Bewegungen jenseits des normalen Ausmaßes, insbesondere in der Kombinationsebene aus Flexion und Supination, für Sprunggelenksverletzungen verantwortlich seien. Die Schlussfolgerung hieraus ist, dass die lateralen Bänder diesen Belastungen am häufigsten ausgesetzt sind. Demnach müsste ein Tapeverbandes diese Strukturen kräftiger stabilisieren als die Medialseitigen. 
Das Belastungsmuster beim Hochspringer strapaziert im Gegensatz zum empirischen Belastungsmuster eher die medialseitigen Bänder. Die Mechanismen, die eine Tapeanlage zur Stabilisation nutzt, könnten nach HUME (1998) die folgenden sein:

1 Widerstand gegen die endständige Beweglichkeit, was letztendlich zu einer verminderten Belastung der Bänder führt.

2 Erhöhung der Muskelfaserrekrutierung, welche die Last auf die Bänder vermindert.

3 Das Gefüge Tape-Haut-Bindegewebe könnte eine Umverteilung der wirkenden Kräfte weg vom Gelenk bewirken.

4 Optimierung des Tibio-Calcanearen Winkels im Sinne einer neutralen Begradigung mit konsekutiver Minderung der pathologischen Supinationsgefahr.

5 Durch die verminderte ROM ist die benötigte Kraft zum Erreichen der endständigen ROM verringert.

6 Durch die verminderte ROM sind die ebenfalls endständigen Winkel im Sprunggelenk vermindert, wodurch wiederum längere Hebelverhältnisse vermieden werden. Kürzere Hebelverhältnisse bedeuten kleinere Kraftresultierende.

7 Schlichtweg durch eine mechanische Verstärkung der Ligamente durch das Tapematerial, welches den Verläufen der Bänder angepasst ist. 
MCLEAN (1989) erörtert verschiedene Erklärungen als verantwortlichen Wirkungsmechanismus von Tape und erklärt, dass adhäsive Tapebinden durchaus als eine Art zweites Ligament fungieren können, insbesondere wenn sie an Hautbereiche anliegen, die relativ unbeweglich gegenüber ihres Untergewebes sind. Somit würde das Tape sich derart anlegen, dass extreme Winkel in der Beweglichkeit stark eingeschränkt würden. Ergänzend hierzu postuliert er, dass weder das Tapematerial, noch das Tape-Haut-Gefüge isoliert jeweils für sich den geschätzten traumatischen Kräften, die zum Riss ligamentärer Strukturen führen würden, standhalten könnten. Kombiniert mit den bindegewebigen Körperstrukturen (subkutanes Fettgewebe, Faszien, etc.) jedoch, verbessert ein Verband die physikalische Eigenschaft der Verteilung von potentiell traumatischen Kräften auf alle beteiligten Strukturen.

Sicherlich spielt auch die Beschaffenheit und Qualität eines Tapes bei allen oben genannten stabilisierenden Mechanismen eine tragende Rolle. So haben Belastungstests von ANDREASSON (1983) erbracht, dass die von ihm untersuchten Tapematerialien bei einer Zugbelastung von $75 \mathrm{~N}$ pro cm Tapebreite zerreißen. Das heißt, dass die beim Sprunggelenkstape eingesetzten Tapes von 2,5 cm Breite der Zerreißprobe bis zu einer Last von $187 \mathrm{~N}$ standhalten würden. An beiden Enden aufgespannt und mittig belastet, hält die Elastizität elastischer Tapes eine Belastung von 256 N/cm Tapebreite aus, während unelastische Tapes bis zu 1280 N/cm Tapebreite überstehen (St PIERRE, 1983). St PIERRE (1983) führte Belastungstests am vorderen Fibularligament von Leichen durch und fand heraus, dass diese einer Kraft zwischen 60 und $560 \mathrm{~N}$ standhalten können bevor sie reißen. Diese Erkenntnis beweist, dass die Festigkeit von Tape insgesamt unter der des vorderen Fibularligament liegt. HUME (1998) fordert in diesem Zusammenhang, dass die Reißfestigkeit von Tapematerial über der von menschlichen Ligamenten liegen müsste, um eine verletzungspräventive Schutzfunktion zu bieten. Unserer 
Ansicht nach, besteht auch schon bei dieser Konstellation, durch die Addition der einzelnenStrukturen (Tape, Gewebe und Ligament) eine gewisse Verstärkung und somit eine Erhöhung der Reißfestigkeit des Gesamtgefüges.

GREENE (1989) schließt aus seiner Studie, dass Tape durchaus in der Lage sei, endständige Beweglichkeit in der Supination-/Pronationsebene selbst nach 20 Minuten sportlicher Belastung einzudämmen, ohne die maximale Kraftentfaltung in derselben Ebene zu beeinflussen.

Mit der Fragestellung inwiefern ein Tapeverband verletzungspräventiv auf die lateralen Bänder des Sprunggelenkes wirkt, recherchierte FIRER (1990) die Literatur und kam zu dem Ergebnis, dass Tape durchaus vor einer Verletzung schützen kann, der zugrunde liegende Mechanismus hierzu jedoch nicht klar ist. Die mechanischen Faktoren, die in der Literatur als verantwortlich postuliert wurden und von GRIGG (1994) weiterhin zugrunde gelegt wurden, ließen durch Belastung nach (z.B. Dehnung des Tapes). Die verstärkte Aktivierung von Muskelgruppen wurde in der Literatur als der Haupteffekt des Tapes beschrieben. FIRER stellte drei zentrale Fragen, welche bis heute noch nicht adäquat geklärt zu sein scheinen:

1 Ist es wissenschaftlich erwiesen, dass Tape die Wahrscheinlichkeit oder das Ausmaß einer Verletzung verringert?

2 Welches sind die zugrundeliegenden Mechanismen?

3 Kann Taping indirekt zu Sekundärschäden führen? 
1993 nahm sich PARIS der ersten Frage an und untersuchte die Literatur in Bezug auf wissenschaftliche Belege der Sprunggelenksstabilität unterschiedlicher Mittel mit besonderer Berücksichtigung der Kraft (isometrische Kraft in Supination und Pronation) und der motorischen Leistung (wie Schnelligkeit, Gleichgewicht, Beweglichkeit und vertikale Sprunghöhe. In seiner Arbeit berichtet FIRER ebenfalls über Literatur, die den Verschleiß, die Handhabung und Tragekomfort, sowie pekuniale Faktoren berücksichtigen. In seiner Zusammenfassung kommt er zu dem Schluss, dass gängig genutzte Orthesen durchaus eine vertretbare Alternative zum Tapeverband darstellen; dass man jedoch zur Auswahl der angemessenen Prophylaxe, nicht nur auf Budget und präventive Qualitäten achten sollte, sondern ein besonderes Gewicht auf den wissenschaftlichen Beleg über den Einfluss der einzelnen Prophylaxe auf die motorische Leistung legen sollte. Zwei Jahre später räumte er jedoch im Rahmen einer weiteren Literatur vergleichenden Studie kritisch ein, dass die Daten zur Sprunggelenksstabilität grundsätzlich unmittelbar nach Anlegen der Orthese erhoben würden und dies zu unschlüssigen Ergebnissen führte.

Aufgrund der allgemein nicht konkreten Aussagen zum Thema Taping, Stabilisation und Leistungseinfluss, tragen wir FIRERs Forderung einen Schritt weiter und verlangen wissenschaftliche Belege zur sportartspezifischen Belastung.

Während MARTIN (1993) die prophylaktische Stellung des Tapes zunehmend in Frage stellt, da bis dato aktuellere Veröffentlichungen alternative Methoden der Sprunggelenksstabilisation über das Taping werteten, beschreibt NORRIS (1994) zur Rechtfertigung die positiven Aspekte des Tapings. Er kommt nach seiner Literaturrecherche in Hinblick auf Bestandteile, Anwendung und Mechanismen des Tapings zu dem Ergebnis, dass eine mechanische 
Unterstützung durch die Einschränkung der Gelenksbeweglichkeit bzw. durch Erhöhung des Gelenkwiderstandes bewirkt wird. Die funktionelle Optimierung begändet er propriozeptiv mit der senso -motorischen Reaktion (Hautstimulation durch Tape), die einerseits zu einer Verkürzung der muskulären Reaktionszeit und andererseits zu einer Begünstigung der Muskelkontraktion führt. Außerdem schließt er einen Lerneffekt (positive Gewöhnung) nicht aus.

Die von uns ermittelten Daten des Absprunges belegen eine deutliche Verkürzung der einzelnen Phasen innerhalb des Absprunges. Die Begründung sehen wir zwar in erster Linie in einer Fixierung der elastisch in einander greifenden einzelnen Fußkomponenten, schließen aber ebenso eine verbesserte inter- und intramuskuläre Koordination nicht aus. Allerdings wäre zur Festigung dieser Annahme eine simultane Aufzeichnung der gelenkführenden Muskelkontraktionen mittels EMG sinnvoll.

Den Aspekt der Lernkurve bzw. der Gewöhnung halten auch wir für sehr plausibel. Es fehlen uns hierzu jedoch subjektive Langzeiterhebungen.

COFFMANN und MITZE (1989) führten ihre Untersuchungen an nur vier College Basketballspielern und weiteren vier, nicht im Leistungssport aktiven, Collegestudenten durch. Die Probanden durchliefen die Versuchsreihen ohne Tape, mit Tapeanlage und mit dem Aircast ${ }^{\circledR}$ Sports Stirrup ${ }^{T M}$. Ihre Ergebnisse belegten, dass im Vergleich zur Kontrollgruppe, durch Tape die vertikale Sprunghöhe sowie die Sprintgeschwindigkeit über die Länge eines Basketballfeldes deutliche Einbüßen zu verzeichnen hatten. Die getestete Sportorthese führte lediglich zu einer signifikant verminderten Sprintgeschwindigkeit, nicht aber zu einer Beeinflussung der Sprunghöhe. 
Insgesamt werteten die Autoren COFFMANN (1989) den

leistungseinschränkenden Einfluss vom Tapeverband stärker, als den von der Sportorthese ein. Wir sehen bei einer solch niedrigen Probandenanzahl das Problem der mangelnden Validität.

1995 führte die Arbeitsgruppe von MACKEAN eine interessante Studie durch, bei welcher an 11 weiblichen Basketballpielerinnen, neben dem beidbeinigen Vertikalsprung, auch die Treffsicherheit beim Sprungwurf, sowie der Sauerstoff- und Kalorienverbrauch unter Tape, ohne Tape (Kontrolle) und mit drei unterschiedlichen das Sprunggelenk unterstützenden Orthesen getestet wurden. MACKEAN kommt zu dem Ergebnis, dass die Leistung im Allgemeinen durch eine externe Gelenkstütze eingeschränkt wird. Dabei schränkt die Active AnkleTM Orthese die Leistung am geringsten ein. Im Vergleich zur Kontrollreihe ohne Tape, waren die Vertikalsprünge signifikant verringert bei den getapeten Sprüngen. Die Treffsicherheit bei Sprüngwürfen wurde im Vergleich zu ohne Tapeverband, mit dem Anlegen des Tapeverbandes am Sprunggelenk deutlich verbessert. Die Autoren stellten einen signifikant höheren Sauerstoff- und Energieverbrauch unter Nutzung der Orthesen im Vergleich zur Tapeanlage fest. Obwohl wir auch hier die Anzahl N=11 der Probanden zur Aufstellung einer Aussage für zu gering halten, können wir dennoch den Aspekt der Treffsicherheit mit Tape für unsere Überlegungen heranziehen. 
Die beim Sprungwurf nötige Stabilität des Körpers während des Abwurfs wird durch einen adäquaten Absprung vorbereitet und gewährleistet. Durch die Tapeanlage erfahren die Athleten, wohlmöglich durch die verbesserte Propriozeption, eine kontrolliertere Führung zum Absprung und optimieren somit ihre Trefferquote. Bezogen auf die Disziplin des Hochsprunges, kann dies eine verbesserte Körperlage während der Flugphase bedeuten, was vielleicht eine effektivere Nutzung der Sprungkraft zur Überquerung der Latte bewirkt.

Inwiefern ein eingeschränkter Abstoß vom Boden mit Tapeanlage durch eine durch dieselbe bewirkte effektivere Körperlage kompensiert werden kann, bleibt zunächst als Substanz für weitergehende Recherchen dahingestellt.

Der eingeschränkte Abstoß korreliert in unseren Versuchen mit der Bewegungseinschränkung durch die Tapeanlage, sodass wir die eingeschränkte Beweglichkeit (ROM = Range of Motion) hierfür verantwortlich sehen. Allerdings deuten unsere Ergebnisse aus der Sprunghöhenmessung, sowie aus den Auswertungen des Druckprofiles beim Absprung, auf eine sukzessive Abnahme der Stabilisation nach jeder Sprungbelastung.

In dieser Hinsicht gibt es zahlreiche Autoren, die die Wirksamkeit des Tapeverbandes in Abhängigkeit von der Belastung untersucht haben. Während erste Berichte von 1962 die verschiedenen Tapingfiguren einander gegenüber stellten, nehmen die Studien der 80er und 90er Jahre vornehmlich Vergleiche zwischen Tape und Orthesen vor. 
So untersuchte RARICK 1962 die Stabilisation vier verschiedener Figuren, welche direkt auf die Haut aufgetragen wurden: Korbflechte, Korbflechte und Steigbügel, Korbflechte und Fersenblock, sowie Korbflechte, Fersenblock und Steigbügel. Dabei maß er die passive Beweglichkeit der Inversion und Eversion im Sprunggelenk von fünf männlichen Teilnehmern im Alter zwischen 21 und 28 Jahren. Die Beweglichkeit wade zunächst vor und nach Tapeanlage nach Neutral-Null gemessen. Es folgte dann eine zehn minütige Belastungsphase, die aus Läufen, Sprüngen, schnellen Anläufen mit abruptem Abbremsen, sowie aus beabsichtigten In- und Eversionen im Stand bestand. Dann wurden die passiven ROMs erneut bei belassenem Tape gemessen. Die Ergebnisse belegten die höchste Festigkeit der letzteren Kategorie, was wir nicht als Überraschung werten können. In der Arbeit wird eine „Verminderung der Bewegungseinschränkung“ von bis zu 50\% nach Belastung beschrieben. Als Gründe werden hierzu neben der mechanischen Dehnung und Schwächung des Tapematerials, auch die unbeständige Haftung des Tapes auf der Haut durch Schweißabsonderung heran gezogen.

Auch in RARICKS Studie ist die Probandenanzahl $(n=5)$ unserer Meinung nach zu gering gewählt. Ferner wurden die Testreihen alle am selben Tag durchgeführt, was den Standard verfälschen könnte; durch Ermüdung ändert sich neben der Belastungsintensität auch die Bewegungsqualität durch Abnahme der Koordination. Außerdem könnte das subjektive Empfindung am Sprunggelenk durch die wechselnden Tapeanlagen beeinflusst werden. Letztendlich jedoch erscheint die Fragestellung, und damit das Ergebnis, in dieser Veröffentlichung ohnehin als selbsterklärend und keiner separaten Studie bedürfend. 
Im selben Jahr veröffentlichte MALINAs Arbeitgruppe eine ähnliche Studie, in welcher die Beweglichkeit des Sprunggelenkes unter vier Bedingungen getestet wurde: Korbflechte und Steigbügel direkt auf die Haut aufgetragen, Korbflechte und Steigbügel über eine Stockinette (ein zu beiden Enden offener Strumpf, der unter dem Tape getragen wurde, um Hautirritationen zu vermeiden und das Entfernen des Tapes zu erleichtern) aufgetragen, ein so genannter Louisana Wrap (ein direkt auf die Haut aufgetragener selbstklebender elastischer Verband, der im Verlauf der 8-er Figur gleich kommt), und letztlich eine Kombination aus Korbflechte, Steigbügel und Fersenblock. Die Übungen der Belastungsphase bestanden aus „Jumping Jacks“, was bei uns als Hampelmann bezeichnet wird, ferner aus beidbeinigen Hochsprüngen, sowie gehockten Sprungserien, die in die Weite gingen. Außerdem Seitschrittläufe und einem Par Cours Lauf. Diese Belastungsphase dauerte insgesamt 5 Minuten. Im Gegensatz zu RARICK führte MALINA (1962) die Tests für jede der Bedingungen an getrennten Tagen durch. Er untersuchte an zehn männlichen Probanden $(n=10)$ die passive Beweglichkeit im Sprunggelenk vor Stabilisierung, nach Stabilisation und nach Belastung mit belassener Stabilisation.

Seine Ergebnisse belegen einen deutlich geringeren Stabilitätsverlust unter Verwendung der Stockinette im Vergleich zu den übrigen Konditionen. Insgesamt jedoch zeigten alle Stabilisationstechniken durch die Belastung eine signifikante Zunahme der ROM.

MALINAs Versuchsaufbau ist mit der zeitlichen Trennung der Testreihen bemüht einen gewissen Standard zu etablieren um Verfälschungen in den Messreihen zu minimieren. Allerdings werden dabei die Leistungsschwankungen in Abhängigkeit von der Tagesform der Probanden nicht berücksichtigt. Es geht 
auch keine konditionelle Einstufung der einzelnen Teilnehmer aus der Arbeit hervor, welche bei der geringen Probandenanzahl und der recht kurzen Belastungsphase zu extremen Ergebnisschwankungen führen kann.

Nichtsdestotrotz eröffneten MALINA (1962) und RARICK (1962) mit ihren nicht leistungsorientierten Untersuchungen als Pioniere die Diskussion um die Wirksamkeit des Tapeverbandes.

1973 veröffentlichte FERGUSON eine Arbeit, in der er die Anwendung des Tapeverbandes nicht nur in Frage stellte, sondern sogar die Schädlichkeit dieser belegen wollte. Er postulierte, dass durch die Bewegunseinschränkung durch Tape, ein natürlicher Schutzreflex der Muskulatur, welcher normalerweise durch ihre Dehnung getriggert würde, häufig erst gar nicht aktiviert werden könne und es durch diese mangelhafte Rekrutierung von Muskelzellen letztendlich zu einer Atrophie der Muskulatur durch Tapeanlage kommen kann. Seither haben zahlreiche Arbeiten wissenschaftlich belegen können, dass es unter Tape zu keiner verminderten Feuerungsaktivität der Muskulatur kommt. So hat SPRINGINGS (1981) anhand elektromyographischer Messungen keine Unterschiede in der Feuerungsaktivität des Peronäusmuskels während eines provozierten Umknickens (rückwärts inversion) des Fußes mit und ohne Tape feststellen können. Das Auftreten auf eine nur $30^{\circ}$ Schräge führte mit Tape zum gleichen Aktivitätsreflex der Peronäusgruppe, wie ohne Tape.

Ebenfalls 1981 veröffentlichten FUMICH et al. ihre Studie an 16 College Athleten (American Football), welche die Bewegungsausmaße des Sprunggelenkes mit Tape, als Maß für den Widerstand, und dieses wiederum als Ausdruck der Stabilisation, denen ohne Tape gegenüber stellte. In dieser Studie 
wurde die Trainingszeit von 2.5 bis 3 Stunden als Belastungszeit definiert. Auch hier ohne weitere Berücksichtigung der individuellen Spielstärke, Gewicht, Netto-Belastungszeit (abzüglich der Pausen) oder Art der tatsächlichen Belastung (Übungen, Position des jeweiligen Spielers, etc.). Es erfolgten dann die Messungen der ROMs, die eine Bewegungseinschränkung von $10^{\circ}$ bis zu $14^{\circ}$ belegten. Dabei wurde die größte Stabilisation in der Inversion, der Plantarflexion und in der Kombinationsbewegung Inversion und Plantarflexion gemessen. Die mechanische Belastung führte zu einer deutlichen Lockerung des Tapes und somit zu einer Zunahme der Bewegungsausmaße um ca. 50\% (durchschnittlich $6^{\circ}$ ). Dennoch bestand aber eine gewisse Stabilisation gegenüber dem ungetapeten Sprunggelenk, sodass die Autoren in ihrem Fazit einen vorteilhaften Effekt des Tapes nicht ausschließen.

LAUGHMAN et al. (1980) führten an 10 gesunden männlichen und 10 gesunden weiblichen Probanden $(n=20)$ im Alter zwischen 20 und 45 Jahren eine ähnlich ausgerichtete Studie durch. Es wurden die Bewegungsausmaße vor und nach Tapeverband, sowie nach Belastung erfasst. Diese bestand aus einem 15 minütigem Dauerlauf entlang einer Achterfigur ohne detaillierte Spezifizierung, und aus 20 „toe raises“ (Zehenheber), welche fälschlicherweise die Plantarflexion an einer $15 \mathrm{~cm}$ hohen Kante (Fersenheber $=$ „Heal raises) beschreiben. Hierbei vermissen wir insbesondere die Laufgeschwindigkeit der Probanden, welche in Verbindung mit dem Kurvenradius extreme Unterschiede der effektiven Belastung ausmachen kann. Die ROM wurde in der dreidimensionalen Supination-Plantarflexions-Ebene gemessen und resultierte in einer Stabilitätsabnahme des Tapeverbandes nach Belastung um mehr als 30 \% (von 26.7\% auf 18.6\%). Die Einschränkung des Bewegungsausmaßes durch Tape von fast $30 \%$ (26.7\%) vor der Belastung wird von den Autoren als Maß der Stabilisation zugrunde gelegt. Dadurch interpretieren sie den Stabilitätsverlust als statistisch signifikant, um den Tapeverband als externe Stabilisation abzulehnen. 
HUGHES et al. untersuchten 1983 im Vergleich das einseitige Taping und die einseitige Schienung als Stabilisation im Sprunggelenk des Sportlers, indem mit einem Leighton $₫$ Flexiometer der Ausmaß der Inversion (Supination) gemessen wurde. Die Probanden wurden hierzu randomisiert einer Links-Fuß und einer Rechts-Fuß-Gruppe zugeordnet, um einer Wertverfälschung durch Dominanz in der Belastung einer Seite entgegen zu wirken. Die 20 minütige Belastungsphase bestand aus Sprint-, Vor- und Rückwärts-, Seitwärtsläufen und einem 1.25 Meilen „Run-Walk“ (Geh-Lauf). Als Resultat stellten die Autoren keine signifikanten Unterschiede in Stabilisation und Abnahme der Stabilisation zwischen der Schienung und Tape. Auch hier sind die Kriterien zur Untersuchung zu vage gefasst. Auffällig ist auch, dass keine expliziten Vergleiche zwischen prä- und post-Stabilisation oder prä- und post-Belastung formuliert und diskutiert werden. Insgesamt eine schwach ausgelegte Studie mit unzureichender Dateninterpretation.

MYBURGH (1984) verglich die Stabilität des Tapeverbandes mit der einer Orthese, indem er bei 12 Squashspielern nach Anlage eines Tapeverbandes die Beweglichkeit des Sprunggelenkes vor und nach einer einstündigen Belastung testete. Dabei wurde der Tape am linken Fuß und die Orthese am rechten Fuß angelegt und die ROM mittels eines Elektrogoniometers im Sitzen erfasst. Auch hier erbrachten die Ergebnisse die Erkenntnis, dass der Tapeverband nach Belastung eine Bewegungseinschränkung von nur noch $10 \%$ bot, während sie vor der Belastung bei bis zu 40\% der freien ROM ohne Tape betrug. Ihre Aussage, dass der Tapeverband seine Wirkung durch eine Belastungsphase verliert, wurde auch von HOCHMAN (1982) in einer einfachen mechanischen Studie bestätigt. Dabei wurde die ROM der Sprunggelenke auf einem Kippbrett, ähnlich einem Therapiekreisel, bestimmt. 
Etwas elaborierter in Ansatz und Durchführung sind jene Studien von VAES (beide 1985), die Röntgenkontrollen, EMG-Messungen und den Einsatz von INMANN (ähnlich dem BIODEX Gerät) Apparaten beinhalteten. In diesen Studien wurde die passive Kippung des Os Talus unter Tapeanlage vor und nach Belastung gemessen. Zur Untersuchung der Wirksamkeit des Tapeverbandes wurden 51 Sprunggelenke mit klinischer Instabilität im oberen Sprunggelenk herangezogen. Die Resultate zeigten trotz deutlicher Abnahme der Stabilität nach der Belastungsphase, dennoch eine signifikante Stabilisation im oberen Sprunggelenk.

Diese Erkenntnis wirft die Frage auf, in welchen Winkelbereichen des Bewegungsausmaßes eine für die Bewegung spezifische Kraftübertragung stattfindet.

Während die normale ROM nach Neutral-Null für die Dorsiflexion / Plantarflexion bei $25 / 0 / 50^{\circ}$ liegt (MILLER, 1985), wird das Sprunggelenk laut MCPOIL und KNECHT (1985), beim normalen Gehen nur bis zu $10^{\circ}$ in die Dorsiflexion und bis zu $20^{\circ}$ in die Plantarflexion bewegt. Beim Lauf erweitern sich diese Bewegungsausmaße auf ca. $20^{\circ}$ Dorsiflexion und bis zu maximal $25^{\circ}$ in die Plantarflexion (SOUTAS-LITTLE et al., 1987). SJOSTROM belegte schon 1978, dass der maximale Impuls beim Abstoß vom Boden im Durchschnitt bei ungefähr $25^{\circ}$ Plantarflexion stattfindet. Diese Ergebnisse wurden von GERDLE (1988) bestätigt.

Diese Resultate zeigen, dass die passive ROM sich zwar über $75^{\circ}$ erstreckt, wir aber unter aktiver Belastung, selbst für einen Absprung, nur maximale $45^{\circ}$ in der Dorsiflexions- / Plantarflexions-Ebene (60\% der freien passiven ROM) in 
Anspruch nehmen. Als Schlussfolgerung ließe sich behaupten, dass eine aktive ROM von über $45^{\circ}$ in der beschriebenen Ebene, unter Belastung als unphysiologisch zu werten wäre.

Die von Autoren wie MYBURGH beschriebene Bewegungseinschränkung von $40 \%$ bedeutet eine verbleibende 60\%ige Beweglichkeit der freien passiven ROM unter Tapeanlage vor Belastung. Diese sollten keinen Einfluss auf die Leistungsentfaltung haben; jedenfalls nicht aufgrund einer Bewegungseinschränkung durch den Verband.

Demnach scheint Tape für den leistungsorientierten Ansatz, lediglich unter kontrollierten Bedingungen eine Leistunseinschränkung zu bewirken, im Gesamtgefüge einer komplexen physiologischen Bewegung jedoch nicht.

Ferner stellt sich die Frage nach der passiven oder aktiven Bewegungsebene als Ursache für das Ausleiern des Verbandes, wenn die Dorsiflexion und Plantarflexion als Ursache weitesgehend ausfallen. Es bleiben hierfür nur noch die Supinations-, Pronationsbewegung, sowie Torsionsbewegungen und Verschiebungen zwischen Laufunterlage bzw. Schuhwerk, Tape und Haut des Athleten zur Erkundung offen.

Die Überlegungen rund um den Einfluss des Tapeverbandes auf die Leistung im Sport, setzt drei Hypothesen voraus: Tape hat keinen Einfluss auf die Leistung, Tape wirkt leistungssteigernd, Tape wirkt leistungsmindernd. Entsprechend lässt sich die Vielzahl von Arbeiten gemäß ihrer primären oder sekundären Resultate in drei Hauptkategorien aufteilen. 
Es besteht kein Zweifel über den Verlust des stabilisierenden Effektes von Tape nach schon wenigen Minuten dynamischer Belastung (PERLMAN 1987). Um so mehr ermutigt diese Tatsache uns zur Schlussfolgerung, dass der Zug des Tapeverbandes an der Haut von Fuß und Unterschenkel vom Menschen zur Beurteilung der Gelenk- bzw. Fußstellung genutzt wird.

Während LINDENBERGER (1985) über den stabilisierenden Effekt von Tapeverband im Sinne einer signifikanten Prophylaxe gegen Außenbandverletzungen berichtet, vertritt BOCCHINFUSO (1994) mit seinen Untersuchungen den indifferenten Standpunkt, dass ein externer Stabilisator keinen signifikanten Einfluss auf die Leistung hat. Im Rahmen einer Veröffentlichung zum Thema der Kosten-Nutzen-Bilanz von Tape, vermuten JEROSCH et al. (1996) anhand ihrer Überlegungen und Untersuchungen, dass die Anlage einer externen Stabilisierung, wie Tape, eine Vorbeugung von geschätzten 30 Bänderverletzungen pro 1000 Einsätzen sportlicher Natur bewirkt. Unserer Meinung nach ist diese Aussage für unsere Maßstäbe sehr vage gefasst und bedarf einer konkreteren Darlegung. So existiert Literatur von verschiedenen Autoren, welche im Versuch wirksame Methoden zur Verletzungsprophylaxe des Sprunggelenkes zu bestimmen, unterschiedliche präventive Trainingsprogramme untersucht haben. Einer dieser Untersuchungen wird von EKSTRAND (1983) vorgestellt. Dabei werden 180 männliche Fußballspieler neben der Versorgung mit Tape, guter Ausrüstung und Information über verletzungsprophylaktisches Verhalten im Sport, auch besonders eingehend trainiert und in der Einhaltung der Maßgaben überwacht. Diese Maßgaben beziehen sich auch auf die Durchführung einer konsequenten Rehabilitation nach stattgefundener Verletzung. Ferner wurden strenge Kriterien zum Ausschluss vom Training bzw. Wettkampf zugunsten einer Verletzungsprophylaxe implementiert. Laut seiner Ergebnisse darf durch eine solche multifaktorielle Versorgung mit einer Reduzierung von Verletzungen um 
75\% rechnen. Allerdings hat dies für uns ebenfalls nur einen bedingten Aussagewert, da der Einsatz von Tape nur eine von sieben Faktoren darstellt. Außerdem werden in EKSTRANDs Studie nur Spieler mit Tape versorgt $(n=52)$, die entweder anamnestisch eine Sprunggelenksverletzung oder ein klinisch instabiles Sprunggelenk vorweisen können. Daher wird die verletzungsprophylaktische Wirkung von Tape auf das gesunde Gelenk nicht untersucht. Ein weiterer Kritikpunkt ist das Fehlen jeglicher Information hinsichtlich des Schuhwerks sowie der genauen Verletzungsdiagnose.

TROPP et al. belegen 1984 und 1985 im Rahmen zweier Studien, dass ein gezieltes Training der Propriozeption und Koordination ebenso reduzierend auf das Verletzungsrisiko sein kann, wie eine Sprunggelenksorthose als externer Stabilisator. Hierbei sind 25 männliche schwedische Fußballspieler einbezogen. Sicherlich ist diese Aussage mindestens genauso imponierend wie interpretationsabhängig. So sehen wir in der gleichen Aussage die Schlussfolgerung, dass propriozeptives Training keinen Vorteil gegenüber externer Stabilisierung bietet. Des Weiteren können wir die darin beschriebenen „signifikanten Unterschiede“ bei einer solch niedrigen Anzahl an Probanden $(n=25)$ unter den gegebenen nicht standardisierten Versuchsbedingungen als sehr unwahrscheinlich und deshalb nicht als valide werten.

Eine weitere Aussage der Studien von GARRICK erklärte schon 1973, dass völlig ungeachtet präventiver Maßnahmen, Spieler mit anamnestisch vorliegender Sprunggelenksverletzung einem höheren Wiederverletzungsrisiko ausgesetzt sind, als bislang unverletzte Spieler. Sie werten die externe Stabilisierung damit gegensätzlich zu TROPPs Schlussfolgerung, dass es bei vorverletzten Spielern einen deutlichen protektiven Einfluß hat, während gesunde Spieler, 
d.h. ohne vorverletztem Sprunggelenk, keinen protektiven Nutzen von einem Tapeverband erwarten können.

Ersteres wird von SHARPE 1997 im Rahmen einer Untersuchung an vorverletzten Fußballerinnen bestätigt. Dabei wurden die Akten von universitären Spielerinnen retrospektiv über einen Zeitraum von fünf Jahren ausgewertet. Spielerinnen, die eine ein- oder beidseitige Sprunggelenksverletzung (56 verletzte Sprunggelenke an 38 Spielerinnen) aufwiesen, erfültten die Einschlusskriterien für die darauf folgende Studie: Jede der vorverletzten Sprunggelenke erhielt randomisiert eines von vier externe Stabilisatoren: 1. zusammenschnürbare Canvas Bandage $(n=19), 2$. Tapeverband $(n=12), 3$. Kombination aus Bandage und Tapeverband $(n=8)$ und 4. keine externe Stabilisierung $(n=17)$. Alle Gruppen erfüllten ein vergleichbares Trainings- und Wettkampfspensum von insgesamt 1717 Trainingseinheiten und 650 Wettkämpfen. Die Rekurrenz von Verletzungen in der 1. Gruppe betrug $0 \%$ und war damit deutlich niedriger als die 2. Gruppe (Tape) mit 25\%. Ebenso viele Rezidivverletzungen passierten in der 3. Kombinationsgruppe (25\%). In der Gruppe 4, ohne jegliche externe Unterstützung, registrierte SHARPE mit 35\% die höchste Rezidvrate an Sprunggelenksverletzungen. Obschon es sehr plausibel erscheint, dass ein unstabilisiertes vorverletztes Sprunggelenk sicherlich einem größeren Wiederverletzungsrisiko ausgesetzt ist, als ein getapetes oder bandagiertes Sprunggelenk, fehlen uns, über diesen Vergleich hinaus, auch in dieser Studie die detaillierten Angaben über Können, Position und effektiver Belastung der jeweiligen Athletinnen, sodass wir für unsere Untersuchungen keine zuverlässige Aussage bezüglich der Stabilität von Tapeverbänden gewinnen können. 
Diese Beobachtungen als Maß nehmend für die Stabilisation durch Tape, können wir, neben den bisher aufgezählten Autoren, auch die Ergebnisse von ROVERE (1988) und SITLER (1994) zur Stützung unserer Thesen heranziehen.

Nachdem also aus den Recherchen hervorgeht, dass die Wirksamkeit vom Tapeverband abhängig von der Sprunggelenksstabilität und / oder gegebenenfalls von einer vorbestehenden Verletzung des Gelenkes abhängig ist, konnten wir keine Arbeit finden, die die Faktoren Schuhwerk, Oberflächenbeschaffenheit des Untergrundes und Bewegungsmuster der Belastung, der Stabilisierung durch Tape in Beziehung zu einander stellt.

Ebenso existiert keine Literatur, welche Art und Umfang einer Sprunggelenksverletzung definiert und damit die Tapeanlage wertet.

Bezüglich der Tapingtechnik gibt es eine Vielfalt von Figuren und Verläufe von Anker und Zügel. Letztendlich jedoch gibt es, wie schon weiter oben erläutert, keine klare Empfehlung hinsichtlich der Kombination der einzelnen Figuren miteinander, sowie deren Häufigkeit. Da es keine vergleichenden Untersuchungen von unterschiedlichen Tapeanlagen, läßt sich auch kein Standard im Taping empfehlen.

Über Qualität des Tapeverbandes, ob es komplett direkt auf die Haut oder durch ein so genanntes Pre-Tape (zum Schutz der Haut beim Entfernen des Tapes) teilweise von der Haut getrennt aufgetragen werden soll, bestehen auch keine Empfehlungen. 
HUME (1998) führt einen weiteren, in der restlichen Literatur unbehandelten Punkt an, der die Frage nach der zeitlichen Länge und der Art der Belastung unbeantwortet läßt. HUME fordert diesbezüglich genauere Untersuchungen, die aber unserer Meinung nach geradezu unmöglich zu beantworten sind. Faktoren wie Koordination, Laufstil, Kraft und die psychologische und motorische Tagesform sind nicht konstant, individuell so unterschiedlich und innerhalb eines Probanden, ja sogar innerhalb des Tapers, extremer Variabilität unterworfen. Beispielsweise erscheint es sehr abwegig, dass ein und derselbe Therapeut den Tapeverband zwei mal exakt identisch unter gleicher Spannung (Zugkraft) anbringt. Andererseits kann ein und dieselbe Tapeanlage (theoretisch) von ein und demselben Athleten an zwei unterschiedlichen Tagen unterschiedlich bewertet werden und folglich seine Leistungsbereitschaft bzw. -fähigkeit beeinflussen (BERIAU, 1994). Eine Untersuchung und Standardisierung dieser Elemente, wie auch von PASSARELLO (1994) gefordert, sehen wir, aufgrund der Variabilität der Einflüsse, letztendlich als ein Unterfangen an, die keine in die Realität umsetzbare Konsequenz bieten kann.

Wie BERIAU (1994), haben auch wir unsere Probanden einen Fragebogen ausfüllen lassen, der die subjektiv empfundene Stabilisierung und Leistungseinschränkung, neben der Bequemlichkeit des Tapeverbandes erfasst hat.

Hinzu kommen die unterschiedlichen Belastungsmuster verschiedener Sportarten, sodass man die Untersuchungen nicht nur sportartspezifisch, sondern jede Bewegung isoliert betrachten muss. Diesem Vorsatz nachgehend, haben wir den komplexen Hochsprung in seine einzelnen Bewegungs- und Belastungskomponenten zerlegt und diese gesondert untersucht. Obwohl unsere Versuche streng leistungsorientiert aufgebaut sind, proklamieren wir 
diesen als den eher Erfolg versprechenden Ansatz zur Annäherung an die von HUME erwarteten reellen Fakten.

Der Einfluss des Tapes auf die sportliche Leistung im Wettkampfsport ist ein kritisch abzuwägender Punkt (VERBRUGGE, 1996). Obwohl inzwischen die Allgemeinheit in der Szene die Meinung vertreten kann, dass eine externe Stabilisierung durch Tape das Verletzungsrisiko herabsetzen kann, gibt es dennoch Sportler, die den Tapeeinsatz aus Sorge vor einer Leistungsminderung ablehnen. Ganz besonders verbreitet ist die Abneigung des Sportlers gegenüber von Orthesen und Sportschienen (PIENKOWSKI, 1995).

Eine sehr wichtige Frage bezüglich des Tapes, welche auch im Mittelpunkt dieser Arbeit steht, ist, ob und inwiefern der Tapeverband einen leistungssteigernden Effekt besitzt. Auch zu diesem Thema äußern sich die Autoren zwiespältig. Während Autoren, wie BUCCINFUSO 1994; GROSS 1994, 1997; MACPHERSON 1995; MACPHERSON et al. 1995; PIENKOWSKI 1995; VERBRUGGE 1996; WILEY \& NIGG 1996; JEROSCH 1997; HUANG 1994 und LOCKE 1996 keinen Leistungsvorteil bringenden Effekt des Tapes nachweisen konnten, sind COFFMANN 1989; GREENE 1990; BURKS 1991; PARIS 1992; BERIAU 1994; MACKEAN 1995; HOCHMAN 1982; METCALFE 1997 der Überzeugung, dass der Athlet durch die Tapeanlage doch einen Leistungsgewinn erwarten kann.

Auch wir sind aufgrund unserer Resultate vom Effekt des Leistungsgewinns, trotz messbarem Stabilitätsverlustes, überzeugt. Bei Betrachtung der Dynamik des letzten Bodenkontakts (Absprung), konnten wir sowohl eine Zunahme der SG-Beweglichkeit nach den fünf Sprüngen, als auch eine Zunahme des Sprungimpulses (Integral der Druckkurve des Absprunges) unter Tape 
feststellen. Wir beobachteten mit Zunahme der Sprungzahl eine Angleichung der Absprungdynamik der getapeten Sprünge an jene Sprünge ohne Tape. Dies deuten wir als eine Abnahme der restriktiven Eigenschaften des Tapes durch die Abnahme der Tapefestigkeit, weniger durch eine erhöhte Krafteinwirkung.

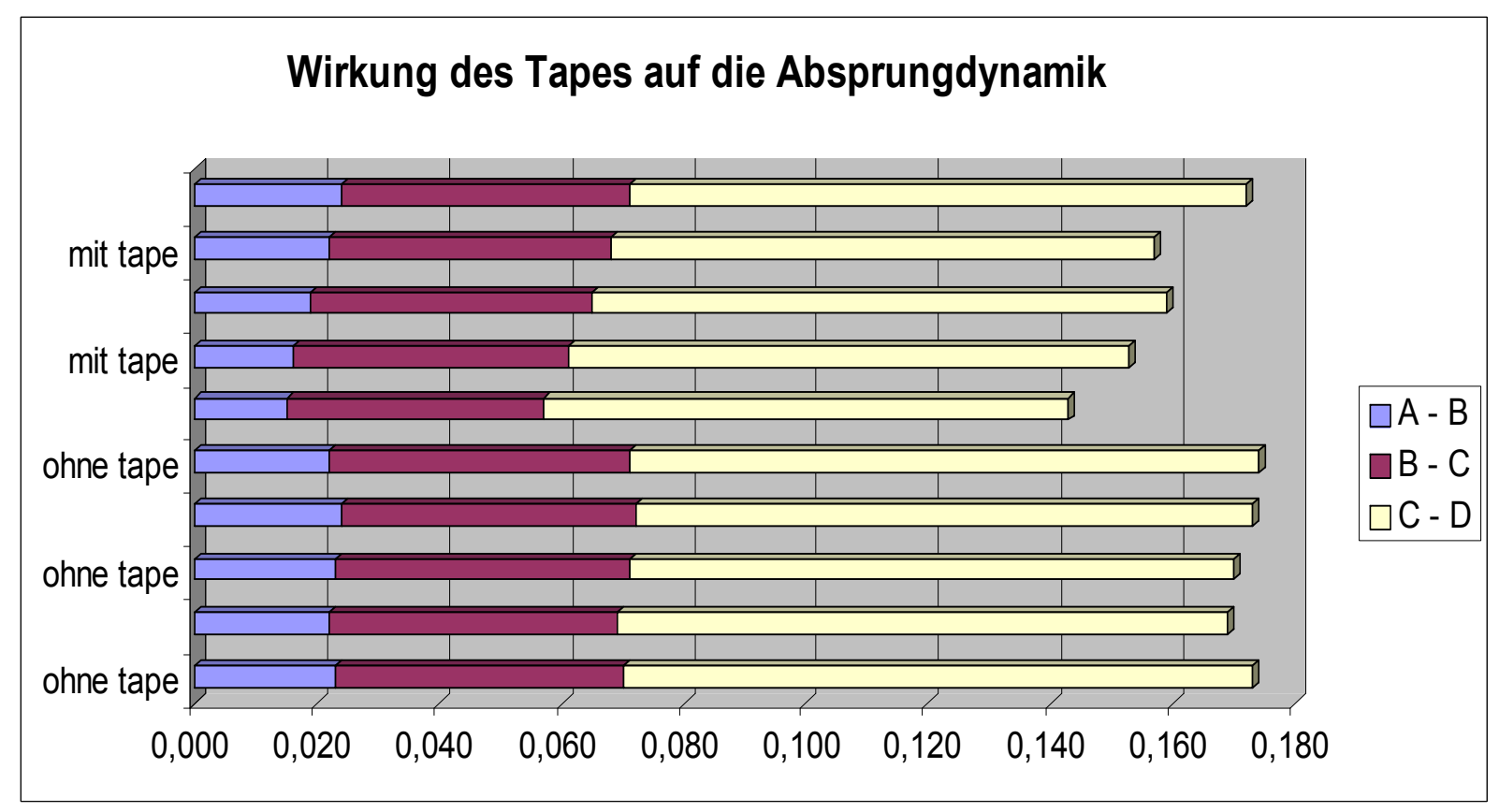

Der kurze Druckabfall während der initialen Standphase des Absprunges dauert ca. 0,015 Sekunden (zwischen B und C). Das schließt einen sensomotorischen Reflexkreis aus, da die Nervenleitgeschwindigkeit der Motoneurone, die schnellsten Neurone unseres Körpers, mit 1 Meter pro Sekunde immer noch zu langsam sind, um diese Reaktion 


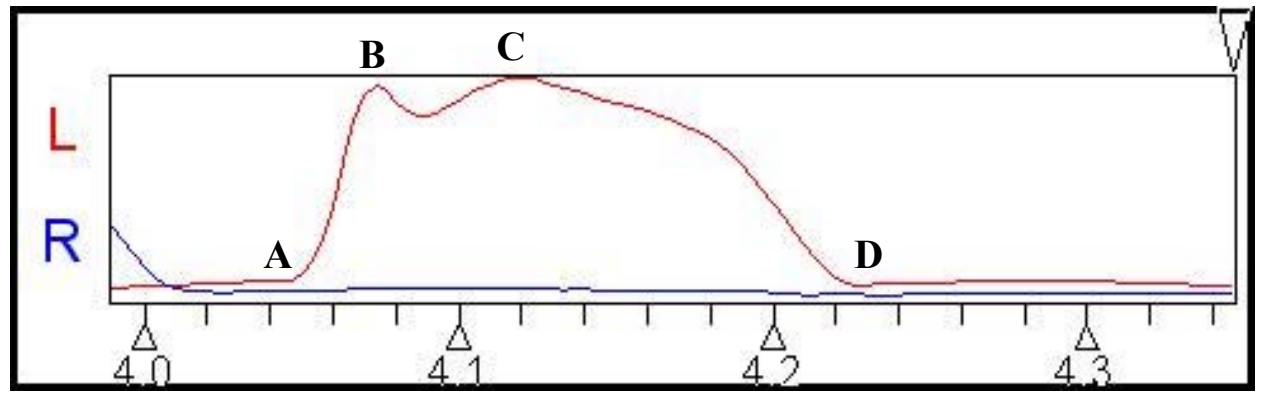

verursachen zu können. Außerdem müsste ein solcher Reflex als polysynaptischer Regelkreis über mindestens zwei Synapsen geschaltet werden, was wiederum Zeit in Anspruch nehmen würde. Eine plausiblere Erklärung hierzu wäre die elastische Rückstoßreaktion aller beteiligten Gewebe und Materialien, welche durch die während der Standphase entstehende Stauchung überschattet wird.

Um den wahren drei-dimensionalen Belastungsvektor während des Absprunges messen zu können, müsste eine Messplatte hergestellt werden, welche zusätzlich zur Y-Achse auch die Druckverhältnisse auf zweidimensionaler Ebene, d.h. X- und Z-Achse, misst. Mit einer Hochgeschwindigkeitskamera (über 150 Bilder pro Sekunde) wäre eine Korrelation der Druckkurve mit dem tatsächlichen Absprung und somit eine aussagekräftigere Auswertung möglich.

Außerdem scheint der Tapeverband, durch die Verhinderung der elastischen Beweglichkeit der einzelnen Fußsegmente untereinander, die einzelnen Phasen zu verkürzen, sodass sie rascher in einander übergehen. Dies würde zweifelsohne bedeuten, dass die weiter proximal gelegenen Gefüge, wie Knie und Hüftgelenk, sowie die Wirbelsäule einer höheren Belastung ausgesetzt sind. Des Weiteren bedeutet die Abnahme der Elastizität auch ein Kompromiss der 
Energiekonservierung im physikalischen Sinn, was sich letztendlich dem Bestreben höher zu springen in den Weg stellen könnte.

Das Sprunggelenk des Sprungbeines muss dabei in der medio-lateralen Bewegungsebene stabil gehalten werden, um einen effizienten Absprung zu gewährleisten.

Eine Instabilität des Sprunggelenkes ist mehr als nur ein mechanisches Problem. Die Wirkung einer externen Stabilisierung kann sowohl mechanisch, als auch propriozeptiv evaluiert werden.

Aus dem klinischen Umfeld weiß man, dass nur 35\% der Patienten mit Sprunggelenksbeschwerden im Sinne einer Instabilität eine Hypermobilität aufweisen (TROPP 1985, VAES 1997). Eine Hypermobilität muss aber nicht zwangsläufig mit einem entsprechenden Beschwerdebild der Instabilität einhergehen. Weitere Testmethoden müssten entwickelt werden, die Faktoren wie Koordination und Propriozeption, neben dem mechanischen Faktor erfassen können.

Propriozeptive Defizite zu erfassen und diese unter getapeten Bedingungen zu untersuchen kann weitere wichtige Informationen über den Effekt eines Tapeverbandes liefern. In unseren Testreihen, in denen ausschließlich gesunde Probanden untersucht worden sind, dienten die funktionellen und dynamischen Tests zur Quantifizierung von propriozeptivem und tatsächlichem Leistungsgewinn durch Tape. 
Insgesamt können wir niederländischen, kanadischen und französischen Publikationen zustimmen, in denen die Wichtigkeit von externer Stabilisierung, wie Tapeverband, für die eine funktionelle Stabilität des Sprunggelenkes anerkannt wird (Conference de consensus $V$ en medecine d'urgence de la societe Francophone d'urgences medicales 1995, NOYES 1977, STIELL 1994).

Aufgrund des in dieser Arbeit gewonnen Erkenntnisse hinsichtlich der propriozeptiven Verstärkung, können wir die Tapeanlage auch für die unmittelbare posttraumatische Phase empfehlen. Damit bestätigen wir ergänzend die von VAES 1998 geäußerten Vermtung, dass eine Tapeanlage nach einer Sprunggelenksverletzung durch einen propriozeptiven Effekt auch zur Stabilität im Gelenk beitrüge.

Taping ist bis zur heutigen Zeit das mit am meisten favorisierte Mittel zur Vorbeugung von Sprunggelenksdistorsionen („Umknick-Verletzung“) im Sport (ROBBINS 1995).

Druck und Dehnung der Haut führt über die Stimulation der darin enthaltenen Mechanorezeptoren zu einer entsprechenden Signalübertragung. GRIGG (1994) legt diesen Mechanismus seiner Erklärung zugrunde, dass neben einer Erfassung von Gelenkbewegung, vielleicht auch unterschiedliche Gelenkstellungen durch Dehnung bzw. Kompression der sie umgebenden Haut wahrgenommen werden können. RIEMANN und LEPHART (2002) modifizieren GRIGGS Aussage dahingehend, dass der postulierte Mechanismus zur Wahrnehmung der Gelenkstellung wahrscheinlich erst in Extremstellungen des Gelenkes zum tragen kommt, während die Wahrnehmung der Gelenkbewegung schon bei kleineren Winkeln signalisiert wird. Dies würde der Funktion der artikulären 
Mechanorezeptoren gleichen. Insgesamt kann in der Literatur jedoch keine klare Erkenntnis über den genauen Mechanismus von Hautmechanorezeptoren gewonnen werden. Fest steht, was SIMONEAU 1997 nochmals unterstrich, dass Hautmechanorezeptoren sowohl die Information über die Bewegung, als auch über die Stellung im Gelenk vermitteln - eine quantifizierende Verteilung der Anteile über diese zwei Funktionen konnte er jedoch nicht aufstellen. RIEMANN und LEPHART sprechen in Ihrer o.g. Arbeit 2002 den afferenten Fasern der Hautmechanorezeptoren eine präzise Sensibilität zur spezifischen Erfassung von Gelenkbewegungen zu.

Zahlreiche Studien dokumentieren den propriozeptiv verstärkenden Effekt von weißem, unelastischen Tape am Sprunggelenk (ANDREASSON 1992, ROBBINS 1995, HEIT 1996, SIMONEAU 1997, etc.).

Auf dem Markt der funktionellen Stützverbände konkurrieren nicht nur Orthesen mit dem ubiquitär bekannten unelastischen weißen Tape. Es finden sich ebenfalls Anbieter von elastischen Tapes, die mit eigener spezieller Technik angelegt, fast schon wundersame Wirkung auf myofasziale Beschwerden bei Sportlern haben sollen. Einer dieser elastischen Produkte wird unter dem Namen Kinesiotape ${ }^{\text {TM }}$ vermarktet und kann, je nach ernannter Indikation, unter Zugspannung angelegt werden. Dadurch wird die Haut aufgewellt und dadurch soll der lymphatische Rückfluss, aber auch die allgemeine Durchblutung bis auf die Muskelebene verbessert sein (www.kinesiotaping.com). Diese

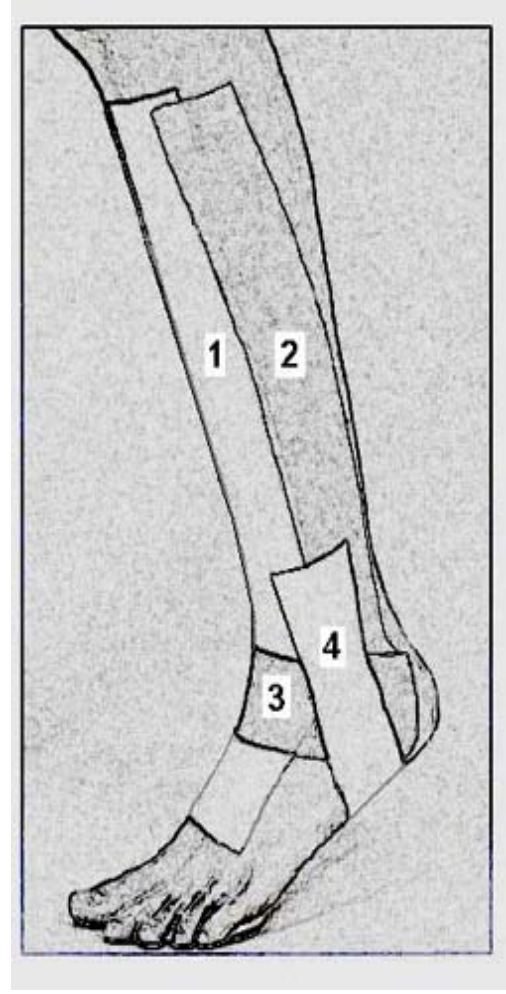

Nur vier Zügel beim Sprunggelenkstape mit Kinesiotape $e^{T M}$ Quelle: HALSETH 2004 
Hypothesen können bislang allerdings nur durch mündliche Empfehlungen namhafter Sportler und allgemeine Anekdoten untermauert werden. MURRAY und HUSK haben 2001 die Auswirkungen von Kinesiotape ${ }^{T M}$ auf die Propriozeption am Sprunggelenk untersucht und daraus eine Verbesserung der Propriozeption in Bezug auf die Gelenkstellung im mittleren Bewegungsradius, in dem die ligamentären Mechanorezeptoren noch nicht rekrutiert sind, gefolgert. Die Ergebnisse beziehen sich allerdings auf den unbelasteten Zustand, sodass auch hier für uns keine echte Praxisrelevanz hergestellt werden kann.

In der Literatur ist der Rückgewinn einer normalen propriozeptiven Funktion nach einer Verletzung des Bewegungsapparates stets das Hauptanliegen im Rahmen der klinischen Rehabilitation gewesen und sollte es weiterhin bleiben (LEPHART et. al. 1997). Insofern erachten auch wir jede Unterstützung in der Verstärkung einer propriozeptiven Funktion, nicht nur in der Rehabilitationsphase, als sehr förderlich. Es bliebe jedoch der direkte objektiv qualifizierende Vergleich zwischen unterschiedlicher Tapingtechniken und verschiedener Tapingmaterialien aus, um eine datenorientierte Bewertung abgeben zu können.

Die aktuellste Studie diesbezüglich liegt von HALSETH und Mitarbeitern vor (2004) und widerlegt die Aussage von MURRAY \& HUSK (2001). Letztere stellen keinen signifikanten Unterschied in der propriozeptiven Leistung bei der Wahrnehmung der Gelenkstellung und -bewegung zwischen Nicht-getapeten und Kinesio-getapeten Probanden fest. Ferner können Sie ebenfalls keine Unterschiede unter den Geschlechtern ( $n=30$ bei 15 Frauen und 15 Männern) nachweisen und bestärken damit die relativ neuen Erkenntnisse von WALTERS aus dem Jahre 2000. 


\section{Zusammenfassung}

Mit unseren Testergebnissen konnten wir die Auffassung bestärken, welche dem Tape eine Verbesserung des propriozeptiven Effektes zuspricht. Diese verbesserte Propriozeption über die intensivierte taktile Reizaufnahme führt wohl zu einem optimierten Sinn für Stellung und Belastung des jeweiligen Körperteiles. Wir behaupten, dass Tape durch diesen Mechanismus zu einer verbesserten Verarbeitung der motorischen Trainingsreize beitragen könnte. Ferner wirkt Tape durch die verbesserte Propriozeption verletzungsvorbeugend.

Darüber hinaus können wir ROBBINS Erkenntnisse auch mit unseren Ergebnissen am Therapiekreisel nicht nur bestätigen, sondern auch, aufgrund des leistungsorientierten und dynamischen Versuchsaufbaues, bestärken.

Den Leistungsgewinn durch Tape konnten wir nicht bei allen Versuchsreihen feststellen. Die komplexe Bewegung des Hochsprunges lässt durch den Einfluss zahlreicher Variablen keine standardisierte Quantifizierung eines tatsächlichen disziplinspezifischen Leistungsgewinnes zu, obwohl eine deutlich höhere Abstoßkraft ausgeübt wird, als ohne Tape. Die subjektive Beurteilung bezüglich des Tapeverbandes gewichtet ebenfalls eindeutig die Favorisierung des Tapes als Stabilisator und Spender von Sicherheitsgefühl. Die Einschränkung der Elastizität während der Absorption führt zu keiner signifikanten Einbuße im Impuls des Absprunges. 
Unserer Auffassung nach bedarf es weiterer biomechanischer Untersuchungen, um den dreidimensionalen Kraftvektor während des Absprunges zu registrieren. Nur so wäre eine deutliche Annäherung an die tatsächlichen Kraftverhältnisse im Sprunggelenk während des Absprunges ermöglicht.

Ferner könnte man durch die exakte Korrelation von Bewegungsstudie und Druckmessung genauere Aussagen über den Winkel der höchsten Beschleunigungskraft im Sprunggelenk treffen und dadurch erfassen, ob und inwiefern der Tape den eigentlichen Absprung behindert.

Abschließend ist die Tatsache hervorzuheben, dass es in der Literatur keinen allgemein postulierten Standpunkt gibt. Dies zeugt von fehlendem Standard in der Untersuchung der Sachverhalte. Wir stimmen mit REISBERG (1992 und 1993) überein, dass die in der Literatur angeführten Studien mitunter die kausalen Zusammenhänge zwar hypothetisch nutzvoll darzustellen imstande sind, deren Methodik und Auswertung jedoch nie mit der notwendigen Relevanz und Konsequenz dargelegt werden. Somit mangelt es in der Forschung um Sprunggelenksorthesen und Tape insgesamt an wissenschaftlichen Daten mit Reliabilität. Der empirische Aussagewert der recherchierten Daten ist daher in Frage zu stellen. 


\section{Anhang}

\section{A - Fragebogen}

Bitte markiere zutreffende Felder

\begin{tabular}{|l|c|}
\hline Meines Wissens, wird & Rehabilitation von Verletzungen \\
Tape im Sport eingesetzt zur & 35 \\
\hline \multirow{1}{*}{} & Prävention von Verletzungen \\
34 \\
\cline { 2 - 2 } \\
\cline { 2 - 2 } \\
\cline { 2 - 2 } \\
\\
Nur vom Arzt oder Physiotherapeuten \\
angelegt \\
22
\end{tabular}

2 Sprungelenk vorverletzt

\begin{tabular}{|c|}
\hline Ja \\
3 \\
\hline Nein \\
32 \\
\hline
\end{tabular}

3 Erfahrung mit Tape am Sprunggelenk

\begin{tabular}{|c|} 
Nie zuvor \\
31 \\
\hline Sporadisch \\
2 \\
\hline Regelmäßig zum Wettkampf \\
\hline 1 \\
\hline Regelmäßig zu Training und Wettkampf \\
\hline
\end{tabular}

4

\begin{tabular}{|l|c|}
\hline Ich benutze kein Tape weil, & Noch nie in Erwägung gezogen \\
32 \\
\cline { 2 - 2 } & Zu teuer \\
1 \\
\cline { 2 - 2 } \\
Zu umständlich \\
2 \\
Ich nicht weiß, wie man es anwendet \\
33 \\
\hline
\end{tabular}

5 Stunden Training pro Woche

\begin{tabular}{|c|}
\hline Unter $5 \mathrm{~h}$ \\
6 \\
\hline Bis $10 \mathrm{~h}$ \\
16 \\
\hline Bis $15 \mathrm{~h}$ \\
9 \\
\hline Über $15 \mathrm{~h}$ \\
4 \\
\hline
\end{tabular}


6

\begin{tabular}{|c|c|}
\hline Tape verleiht mir das Gefühl der ... & Sicherheit \\
\cline { 2 - 2 } & 34 \\
\cline { 2 - 2 } & Stabilität \\
24 & Weder, noch \\
1 & \\
\end{tabular}

7 Den Tapeverband empfand ich als

\begin{tabular}{|c|}
\hline Eher störend \\
9 \\
\hline Leistungsfördernd \\
19 \\
\hline Weder, noch \\
7 \\
\hline
\end{tabular}

8 Gefühl für den Absprung/Übung durch Tape

\begin{tabular}{|c|}
\hline Schlechter \\
23 \\
\hline Besser \\
12 \\
\hline Unverändert \\
0 \\
\hline
\end{tabular}

9

\begin{tabular}{|l|c|}
\hline Nach Entfernung des Tapes & Vorsichtiger \\
, gehe ich ... in den Absprung & 29 \\
\hline & Kraft- und schwungvoller \\
& 3 \\
\cline { 2 - 2 } & Unverändert \\
2
\end{tabular}

10 Nach Entfernung des Tapes Gefühl für den Absprung/Übung ohne Tape Schlechter 27 Besser 3 Unverändert 5

11 Wenn das finanzielle keine Rolle spielte , dann würde ich wahrscheinlich... Trotzdem kein tape benutzen 7 Mich zu jedem Training tapen lassen 28 
B - Phasen des Abdrucks im Durchschnitt aller Sprünge

$\begin{array}{cccccccc} & \text { 1st peak } & \text { Start to PO } & \text { Latency } & \text { TCT } & \text { Plateau } & \text { Acc } & \\ 1 & 0,015 & 0,048 & 0,089 & 0,152 & 0,137 & 0,089 & \mathrm{~T} \\ & 0,023 & 0,047 & 0,103 & 0,173 & 0,150 & 0,103 & \text { no } \mathrm{T} \\ 2 & 0,021 & 0,042 & 0,086 & 0,149 & 0,128 & 0,086 & \mathrm{~T} \\ & 0,016 & 0,042 & 0,072 & 0,130 & 0,114 & 0,072 & \text { no T } \\ 3 & 0,022 & 0,043 & 0,085 & 0,150 & 0,128 & 0,085 & \mathrm{~T} \\ & 0,024 & 0,041 & 0,082 & 0,147 & 0,123 & 0,082 & \text { no T } \\ 4 & 0,030 & 0,044 & 0,113 & 0,187 & 0,157 & 0,113 & \mathrm{~T} \\ & 0,031 & 0,039 & 0,097 & 0,167 & 0,136 & 0,097 & \text { no T } \\ 5 & 0,030 & 0,056 & 0,082 & 0,168 & 0,138 & 0,082 & \mathrm{~T} \\ & 0,030 & 0,048 & 0,093 & 0,171 & 0,141 & 0,093 & \text { no T }\end{array}$




\section{C - Sprünge beidbeinig}

\begin{tabular}{|c|c|c|c|c|c|}
\hline & & & & $\begin{array}{l}\text { avrg } \\
\text { sprunghöhe }\end{array}$ & $\begin{array}{l}\text { diff } \\
\text { sprunghöhe }\end{array}$ \\
\hline \multicolumn{6}{|l|}{01 ohne tape } \\
\hline 0,48 & 0,47 & 0,49 & 0,48 & & \\
\hline 0,28 & 0,27 & 0,29 & 0,28 & 0,28 & \\
\hline \multicolumn{6}{|l|}{01 mit tape } \\
\hline 0,40 & 0,41 & 0,45 & 0,43 & & \\
\hline \multirow{2}{*}{0,19} & 0,21 & 0,25 & 0,23 & 0,22 & \\
\hline & & & & & 0,06 \\
\hline \multicolumn{6}{|l|}{02 ohne tape } \\
\hline 0,46 & 0,46 & 0,48 & 0,48 & & \\
\hline 0,25 & 0,26 & 0,28 & 0,28 & 0,27 & \\
\hline \multicolumn{6}{|l|}{02 mit tape } \\
\hline 0,44 & 0,45 & 0,44 & 0,44 & & \\
\hline \multirow[t]{2}{*}{0,24} & 0,25 & 0,23 & 0,23 & 0,24 & \\
\hline & & & & & 0,03 \\
\hline \multicolumn{6}{|l|}{03 ohne tape } \\
\hline 0,38 & 0,36 & 0,36 & 0,38 & & \\
\hline 0,18 & 0,16 & 0,16 & 0,18 & 0,17 & \\
\hline \multicolumn{6}{|c|}{03 mit tape } \\
\hline 0,34 & 0,37 & 0,36 & 0,36 & & \\
\hline \multirow[t]{2}{*}{0,14} & 0,17 & 0,15 & 0,16 & 0,16 & \\
\hline & & & & & 0,01 \\
\hline \multicolumn{6}{|l|}{04 ohne tape } \\
\hline 0,44 & 0,37 & 0,36 & 0,35 & & \\
\hline 0,24 & 0,17 & 0,16 & 0,15 & 0,18 & \\
\hline \multicolumn{6}{|l|}{04 mit tape } \\
\hline 0,34 & 0,36 & 0,34 & 0,38 & & \\
\hline \multirow[t]{2}{*}{0,14} & 0,16 & 0,14 & 0,17 & 0,15 & \\
\hline & & & & & 0,02 \\
\hline \multicolumn{6}{|c|}{ 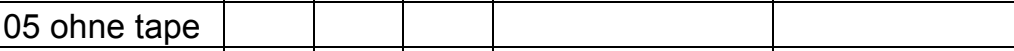 } \\
\hline 0,38 & 0,39 & 0,38 & 0,41 & & \\
\hline 0,18 & 0,19 & 0,18 & 0,20 & 0,19 & \\
\hline \multicolumn{6}{|l|}{05 mit tape } \\
\hline 0,38 & 0,39 & 0,41 & 0,43 & & \\
\hline \multirow[t]{4}{*}{0,18} & 0,18 & 0,20 & 0,22 & 0,20 & \\
\hline & & & & & $-0,01$ \\
\hline & & & & & \\
\hline & & & & & \\
\hline
\end{tabular}




\begin{tabular}{|c|c|c|c|c|c|}
\hline \multicolumn{6}{|c|}{06 ohne tape } \\
\hline 0,51 & 0,53 & 0,49 & 0,53 & & \\
\hline 0,32 & 0,34 & 0,29 & 0,34 & 0,33 & \\
\hline \multicolumn{6}{|l|}{06 mit tape } \\
\hline 0,46 & 0,47 & 0,47 & 0,47 & & \\
\hline 0,26 & 0,27 & 0,27 & 0,27 & 0,27 & \\
\hline & & & & & 0,06 \\
\hline \multicolumn{6}{|l|}{07 ohne tape } \\
\hline 0,42 & 0,40 & 0,44 & 0,42 & & \\
\hline 0,22 & 0,20 & 0,24 & 0,22 & 0,22 & \\
\hline \multicolumn{6}{|l|}{07 mit tape } \\
\hline 0,40 & 0,38 & 0,40 & 0,41 & & \\
\hline 0,19 & 0,18 & 0,20 & 0,21 & 0,19 & \\
\hline & & & & & 0,02 \\
\hline \multicolumn{6}{|c|}{\begin{tabular}{|l|l|l|l|}
08 ohne tape & & & \\
\end{tabular}} \\
\hline 0,48 & 0,47 & 0,53 & 0,48 & & \\
\hline 0,28 & 0,27 & 0,34 & 0,28 & 0,30 & \\
\hline \multicolumn{6}{|l|}{08 mit tape } \\
\hline 0,40 & 0,45 & 0,45 & 0,43 & & \\
\hline 0,19 & 0,25 & 0,25 & 0,23 & 0,23 & \\
\hline & & & & & 0,07 \\
\hline \multicolumn{6}{|l|}{09 ohne tape } \\
\hline 0,33 & 0,37 & 0,37 & 0,39 & & \\
\hline 0,13 & 0,17 & 0,17 & 0,19 & 0,16 & \\
\hline \multicolumn{6}{|l|}{09 mit tape } \\
\hline 0,30 & 0,36 & 0,37 & 0,35 & & \\
\hline 0,11 & 0,16 & 0,17 & 0,15 & 0,15 & \\
\hline & & & & & 0,02 \\
\hline \multicolumn{6}{|l|}{10 ohne tape } \\
\hline 0,49 & 0,47 & 0,55 & 0,53 & & \\
\hline 0,29 & 0,27 & 0,37 & 0,34 & 0,32 & \\
\hline \multicolumn{6}{|c|}{\begin{tabular}{|l|l|l|}
10 mit tape & & \\
\end{tabular}} \\
\hline 0,49 & 0,46 & 0,48 & 0,50 & & \\
\hline 0,29 & 0,26 & 0,28 & 0,31 & 0,29 & \\
\hline & & & & & 0,04 \\
\hline \multicolumn{6}{|c|}{\begin{tabular}{|l|l|l|l|}
11 ohne tape & & & \\
\end{tabular}} \\
\hline 0,44 & 0,47 & 0,49 & 0,48 & & \\
\hline 0,24 & 0,27 & 0,29 & 0,28 & 0,27 & \\
\hline & & & & & \\
\hline \multicolumn{6}{|l|}{11 mit tape } \\
\hline 0,40 & 0,45 & 0,45 & 0,44 & & \\
\hline 0,19 & 0,25 & 0,25 & 0,24 & 0,23 & \\
\hline & & & & & 0,04 \\
\hline
\end{tabular}




\begin{tabular}{|c|c|c|c|c|c|}
\hline \multicolumn{6}{|l|}{12 ohne tape } \\
\hline 0,38 & 0,41 & 0,41 & 0,42 & & \\
\hline 0,18 & 0,21 & 0,21 & 0,22 & 0,20 & \\
\hline \multicolumn{6}{|l|}{12 mit tape } \\
\hline 0,40 & 0,41 & 0,38 & 0,39 & & \\
\hline 0,20 & 0,21 & 0,18 & 0,19 & 0,19 & \\
\hline & & & & & 0,01 \\
\hline \multicolumn{6}{|l|}{13 ohne tape } \\
\hline 0,59 & 0,60 & 0,58 & 0,55 & & \\
\hline 0,43 & 0,44 & 0,41 & 0,37 & 0,41 & \\
\hline \multicolumn{6}{|l|}{13 mit tape } \\
\hline 0,49 & 0,51 & 0,50 & 0,49 & & \\
\hline 0,29 & 0,32 & 0,31 & 0,29 & 0,30 & \\
\hline & & & & & 0,11 \\
\hline \multicolumn{6}{|c|}{\begin{tabular}{|l|l|l|l|}
14 ohne tape & & & \\
\end{tabular}} \\
\hline 0,48 & 0,47 & 0,49 & 0,49 & & \\
\hline 0,28 & 0,27 & 0,29 & 0,29 & 0,29 & \\
\hline \multicolumn{6}{|l|}{14 mit tape } \\
\hline 0,42 & 0,41 & 0,45 & 0,44 & & \\
\hline 0,22 & 0,21 & 0,25 & 0,24 & 0,23 & \\
\hline & & & & & 0,06 \\
\hline \multicolumn{6}{|l|}{15 ohne tape } \\
\hline 0,41 & 0,43 & 0,43 & 0,48 & & \\
\hline 0,21 & 0,23 & 0,23 & 0,28 & 0,23 & \\
\hline \multicolumn{6}{|l|}{15 mit tape } \\
\hline 0,40 & 0,41 & 0,45 & 0,43 & & \\
\hline 0,19 & 0,21 & 0,25 & 0,23 & 0,22 & \\
\hline & & & & & 0,02 \\
\hline \multicolumn{6}{|l|}{16 ohne tape } \\
\hline 0,52 & 0,52 & 0,51 & 0,52 & & \\
\hline 0,33 & 0,33 & 0,32 & 0,33 & 0,33 & \\
\hline \multicolumn{6}{|l|}{16 mit tape } \\
\hline 0,48 & 0,41 & 0,45 & 0,47 & & \\
\hline 0,28 & 0,21 & 0,25 & 0,27 & 0,25 & \\
\hline & & & & & 0,08 \\
\hline \multicolumn{6}{|c|}{\begin{tabular}{l|l|l|l|}
17 ohne tape & & & \\
\end{tabular}} \\
\hline 0,39 & 0,39 & 0,40 & 0,38 & & \\
\hline 0,19 & 0,19 & 0,20 & 0,18 & 0,19 & \\
\hline \multirow{2}{*}{\multicolumn{6}{|c|}{17 mit tape }} \\
\hline & 0.36 & 039 & 037 & & \\
\hline 0,17 & 0,16 & 0,19 & 0,17 & 0,17 & \\
\hline & & & & & 0,02 \\
\hline
\end{tabular}




\begin{tabular}{|c|c|c|c|c|c|}
\hline \multicolumn{6}{|c|}{18 ohne tape } \\
\hline 0,35 & 0,34 & 0,33 & 0,33 & & \\
\hline 0,15 & 0,14 & 0,13 & 0,13 & 0,14 & \\
\hline \multicolumn{6}{|l|}{18 mit tape } \\
\hline 0,35 & 0,35 & 0,31 & 0,31 & & \\
\hline 0,15 & 0,15 & 0,12 & 0,12 & 0,13 & \\
\hline & & & & & 0,01 \\
\hline \multicolumn{6}{|l|}{19 ohne tape } \\
\hline 0,48 & 0,47 & 0,49 & 0,48 & & \\
\hline 0,28 & 0,27 & 0,29 & 0,28 & 0,28 & \\
\hline \multicolumn{6}{|l|}{19 mit tape } \\
\hline 0,40 & 0,45 & 0,47 & 0,43 & & \\
\hline 0,19 & 0,25 & 0,27 & 0,23 & 0,23 & \\
\hline & & & & & 0,05 \\
\hline \multicolumn{6}{|c|}{\begin{tabular}{|l|l|l|l|}
20 ohne tape & & & \\
\end{tabular}} \\
\hline 0,45 & 0,47 & 0,45 & 0,48 & & \\
\hline 0,25 & 0,27 & 0,25 & 0,28 & 0,26 & \\
\hline \multicolumn{6}{|l|}{20 mit tape } \\
\hline 0,40 & 0,44 & 0,45 & 0,43 & & \\
\hline 0,19 & 0,24 & 0,25 & 0,23 & 0,23 & \\
\hline & & & & & 0,04 \\
\hline \multicolumn{6}{|l|}{21 ohne tape } \\
\hline 0,48 & 0,48 & 0,49 & 0,48 & & \\
\hline 0,28 & 0,28 & 0,29 & 0,28 & 0,28 & \\
\hline \multicolumn{6}{|l|}{21 mit tape } \\
\hline 0,41 & 0,41 & 0,45 & 0,43 & & \\
\hline 0,21 & 0,21 & 0,25 & 0,23 & 0,22 & \\
\hline & & & & & 0,06 \\
\hline \multicolumn{6}{|l|}{22 ohne tape } \\
\hline 0,47 & 0,47 & 0,49 & 0,48 & & \\
\hline 0,27 & 0,27 & 0,29 & 0,28 & 0,28 & \\
\hline \multicolumn{6}{|l|}{22 mit tape } \\
\hline 0,42 & 0,41 & 0,45 & 0,43 & & \\
\hline 0,22 & 0,21 & 0,25 & 0,23 & 0,22 & \\
\hline & & & & & 0,06 \\
\hline \multicolumn{6}{|c|}{\begin{tabular}{|l|l|l|l|}
23 ohne tape & & & \\
\end{tabular}} \\
\hline 0,40 & 0,42 & 0,45 & 0,45 & & \\
\hline 0,20 & 0,22 & 0,25 & 0,25 & 0,23 & \\
\hline \\
\hline 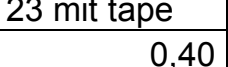 & 0,41 & 0.45 & 0.43 & & \\
\hline 0,19 & 0,21 & 0,25 & 0,23 & 0,22 & \\
\hline & & & & & 0,01 \\
\hline
\end{tabular}




\begin{tabular}{|c|c|c|c|c|c|}
\hline \multicolumn{6}{|l|}{24 ohne tape } \\
\hline 0,48 & 0,47 & 0,49 & 0,44 & & \\
\hline 0,28 & 0,27 & 0,29 & 0,24 & 0,27 & \\
\hline \multicolumn{6}{|l|}{24 mit tape } \\
\hline 0,40 & 0,41 & 0,45 & 0,43 & & \\
\hline 0,19 & 0,21 & 0,25 & 0,23 & 0,22 & \\
\hline & & & & & 0,05 \\
\hline \multicolumn{6}{|l|}{25 ohne tape } \\
\hline 0,55 & 0,52 & 0,52 & 0,56 & & \\
\hline 0,37 & 0,33 & 0,33 & 0,38 & 0,35 & \\
\hline \multicolumn{6}{|l|}{25 mit tape } \\
\hline 0,50 & 0,53 & 0,50 & 0,51 & & \\
\hline 0,31 & 0,34 & 0,31 & 0,32 & 0,32 & \\
\hline & & & & & 0,04 \\
\hline \multicolumn{6}{|c|}{\begin{tabular}{|l|l|l|l|}
26 ohne tape & & & \\
\end{tabular}} \\
\hline 0,46 & 0,47 & 0,49 & 0,46 & & \\
\hline 0,26 & 0,27 & 0,29 & 0,26 & 0,27 & \\
\hline \multirow{2}{*}{\multicolumn{6}{|c|}{26 mit tape }} \\
\hline & & & & & \\
\hline 0,42 & 0,41 & 0,44 & 0,43 & & \\
\hline 0,22 & 0,21 & 0,24 & 0,23 & 0,22 & \\
\hline & & & & & 0,05 \\
\hline \multicolumn{6}{|l|}{27 ohne tape } \\
\hline 0,48 & 0,47 & 0,52 & 0,48 & & \\
\hline 0,28 & 0,27 & 0,33 & 0,28 & 0,29 & \\
\hline \multirow{2}{*}{\multicolumn{6}{|c|}{27 mit tape }} \\
\hline & 0.42 & 0.45 & 0.43 & & \\
\hline$\frac{0,45}{0,25}$ & $\mid$\begin{tabular}{|l|l|}
0,420 \\
\end{tabular} & \begin{tabular}{|l|l|}
0,40 \\
002
\end{tabular} & 0,43 & & \\
\hline 0,25 & 0,22 & 0,25 & 0,23 & 0,24 & \\
\hline & & & & & 0,06 \\
\hline \multicolumn{6}{|c|}{\begin{tabular}{|l|l|l|l|}
28 ohne tape & & & \\
\end{tabular}} \\
\hline 0,48 & 0,53 & 0,49 & 0,48 & & \\
\hline 0,28 & 0,34 & 0,29 & 0,28 & 0,30 & \\
\hline \multicolumn{6}{|c|}{\begin{tabular}{|l|l|l|l|}
28 mit tape & & & \\
\end{tabular}} \\
\hline 0,45 & 0,50 & 0,45 & 0,46 & & \\
\hline 0,25 & 0,31 & 0,25 & 0,26 & 0,27 & \\
\hline & & & & & 0,03 \\
\hline \multicolumn{6}{|c|}{\begin{tabular}{|l|l|l|l|}
29 ohne tape & & & \\
\end{tabular}} \\
\hline 0,50 & 0,47 & 0,49 & 0,48 & & \\
\hline 0,31 & 0,27 & 0,29 & 0,28 & 0,29 & \\
\hline \multicolumn{6}{|l|}{29 mit tape } \\
\hline 0,40 & 0,46 & 0,45 & 0,45 & & \\
\hline 0,19 & 0,26 & 0,25 & 0,25 & 0,24 & \\
\hline & & & & & 0,05 \\
\hline
\end{tabular}




\begin{tabular}{|c|c|c|c|c|c|}
\hline \multicolumn{6}{|l|}{30 ohne tape } \\
\hline 0,48 & 0,47 & 0,49 & 0,48 & & \\
\hline 0,28 & 0,27 & 0,29 & 0,28 & 0,28 & \\
\hline \multicolumn{6}{|l|}{30 mit tape } \\
\hline 0,44 & 0,41 & 0,38 & 0,43 & & \\
\hline 0,24 & 0,21 & 0,18 & 0,23 & 0,21 & \\
\hline & & & & & 0,07 \\
\hline \multicolumn{6}{|l|}{31 ohne tape } \\
\hline 0,48 & 0,47 & 0,49 & 0,48 & & \\
\hline 0,28 & 0,27 & 0,29 & 0,28 & 0,28 & \\
\hline \multicolumn{6}{|l|}{31 mit tape } \\
\hline 0,47 & 0,48 & 0,45 & 0,43 & & \\
\hline 0,27 & 0,28 & 0,25 & 0,23 & 0,26 & \\
\hline & & & & & 0,03 \\
\hline \multicolumn{6}{|c|}{\begin{tabular}{|l|l|l|l|}
32 ohne tape & & & \\
\end{tabular}} \\
\hline 0,48 & 0,47 & 0,49 & 0,46 & & \\
\hline 0,28 & 0,27 & 0,29 & 0,26 & 0,28 & \\
\hline \multicolumn{6}{|l|}{32 mit tape } \\
\hline 0,40 & 0,45 & 0,45 & 0,43 & & \\
\hline 0,19 & 0,25 & 0,25 & 0,23 & 0,23 & \\
\hline & & & & & 0,05 \\
\hline \multicolumn{6}{|l|}{33 ohne tape } \\
\hline 0,48 & 0,47 & 0,49 & 0,48 & & \\
\hline 0,28 & 0,27 & 0,29 & 0,28 & 0,28 & \\
\hline \multicolumn{6}{|l|}{33 mit tape } \\
\hline 0,40 & 0,40 & 0,45 & 0,43 & & \\
\hline 0,19 & 0,20 & 0,25 & 0,23 & 0,22 & \\
\hline & & & & & 0,07 \\
\hline \multicolumn{6}{|l|}{34 ohne tape } \\
\hline 0,48 & 0,47 & 0,49 & 0,48 & & \\
\hline 0,28 & 0,27 & 0,29 & 0,28 & 0,28 & \\
\hline \multicolumn{6}{|l|}{34 mit tape } \\
\hline 0,40 & 0,41 & 0,45 & 0,42 & & \\
\hline 0,19 & 0,21 & 0,25 & 0,22 & 0,22 & \\
\hline & & & & & 0,07 \\
\hline \multicolumn{6}{|c|}{\begin{tabular}{|l|l|l|l|}
35 ohne tape & & & \\
\end{tabular}} \\
\hline 0,48 & 0,47 & 0,49 & 0,48 & & \\
\hline 0,28 & 0,27 & 0,29 & 0,28 & 0,28 & \\
\hline & & & & & \\
\hline \multicolumn{6}{|l|}{35 mit tape } \\
\hline 0,45 & 0,43 & 0,45 & 0,43 & & \\
\hline 0,25 & 0,23 & 0,25 & 0,23 & 0,24 & \\
\hline & & & & & 0,05 \\
\hline
\end{tabular}




\section{D- Sprünge einbeinig}

\begin{tabular}{|c|c|c|c|c|c|}
\hline & & & & $\begin{array}{l}\text { avrg } \\
\text { sprunghöhe }\end{array}$ & $\begin{array}{l}\text { diff } \\
\text { sprunghöhe }\end{array}$ \\
\hline \multicolumn{6}{|l|}{01 ohne tape } \\
\hline 0,34 & 0,38 & 0,38 & 0,37 & & \\
\hline 0,14 & 0,18 & 0,18 & 0,17 & 0,17 & \\
\hline \multicolumn{6}{|l|}{01 mit tape } \\
\hline 0,34 & 0,35 & 0,35 & 0,38 & & \\
\hline \multirow[t]{2}{*}{0,14} & 0,15 & 0,15 & 0,18 & 0,15 & \\
\hline & & & & & $-0,01$ \\
\hline \multicolumn{6}{|l|}{02 ohne tape } \\
\hline 0,39 & 0,38 & 0,46 & 0,47 & & \\
\hline 0,19 & 0,18 & 0,26 & 0,27 & 0,22 & \\
\hline \multicolumn{6}{|l|}{02 mit tape } \\
\hline 0,41 & 0,42 & 0,41 & 0,42 & & \\
\hline \multirow[t]{2}{*}{0,21} & 0,22 & 0,21 & 0,22 & 0,21 & \\
\hline & & & & & $-0,01$ \\
\hline \multicolumn{6}{|l|}{03 ohne tape } \\
\hline 0,34 & 0,36 & 0,38 & 0,36 & & \\
\hline 0,14 & 0,16 & 0,18 & 0,16 & 0,16 & \\
\hline \multicolumn{6}{|l|}{03 mit tape } \\
\hline 0,40 & 0,43 & 0,48 & 0,48 & & \\
\hline \multirow[t]{2}{*}{0,20} & 0,23 & 0,28 & 0,28 & 0,25 & \\
\hline & & & & & 0,09 \\
\hline \multicolumn{6}{|l|}{04 ohne tape } \\
\hline 0,36 & 0,36 & 0,34 & 0,34 & & \\
\hline 0,16 & 0,16 & 0,14 & 0,14 & 0,15 & \\
\hline \multicolumn{6}{|l|}{04 mit tape } \\
\hline 0,40 & 0,43 & 0,38 & 0,38 & & \\
\hline \multirow[t]{2}{*}{0,20} & 0,23 & 0,18 & 0,18 & 0,19 & \\
\hline & & & & & 0,04 \\
\hline \multicolumn{6}{|c|}{\begin{tabular}{|l|l|l|}
05 ohne tape & & \\
\end{tabular}} \\
\hline 0,40 & 0,38 & 0,39 & 0,40 & & \\
\hline 0,20 & 0,18 & 0,19 & 0,20 & 0,19 & \\
\hline \multicolumn{6}{|c|}{\begin{tabular}{l|l|l}
05 mit tape & & \\
\end{tabular}} \\
\hline 0,42 & 0,43 & 0,46 & 0,46 & & \\
\hline \multirow[t]{4}{*}{0,22} & 0,23 & 0,26 & 0,26 & 0,24 & \\
\hline & & & & & 0,05 \\
\hline & & & & & \\
\hline & & & & & \\
\hline
\end{tabular}




\begin{tabular}{|c|c|c|c|c|c|}
\hline 06 ohne tape & & & & & \\
\hline 0,41 & 0,43 & 0,39 & 0,43 & & \\
\hline 0,21 & 0,23 & 0,19 & 0,23 & 0,21 & \\
\hline 06 mit tape & & & & & \\
\hline 0,46 & 0,47 & 0,47 & 0,47 & & \\
\hline 0,26 & 0,27 & 0,27 & 0,27 & 0,27 & \\
\hline & & & & & 0,06 \\
\hline 07 ohne tape & & & & & \\
\hline 0,38 & 0,35 & 0,39 & 0,40 & & \\
\hline 0,18 & 0,15 & 0,19 & 0,20 & 0,18 & \\
\hline 07 mit tape & & & & & \\
\hline 0,40 & 0,38 & 0,40 & 0,41 & & \\
\hline 0,19 & 0,18 & 0,20 & 0,21 & 0,19 & \\
\hline & & & & & 0,02 \\
\hline 08 ohne tape & & & & & \\
\hline 0,37 & 0,37 & 0,43 & 0,38 & & \\
\hline 0,17 & 0,17 & 0,23 & 0,18 & 0,18 & \\
\hline 08 mit tape & & & & & \\
\hline 0,40 & 0,45 & 0,45 & 0,43 & & \\
\hline 0,19 & 0,25 & 0,25 & 0,23 & 0,23 & \\
\hline & & & & & 0,04 \\
\hline 09 ohne tape & & & & & \\
\hline 0,33 & 0,37 & 0,37 & 0,39 & & \\
\hline 0,13 & 0,17 & 0,17 & 0,19 & 0,16 & \\
\hline 09 mit tape & & & & & \\
\hline 0,34 & 0,38 & 0,38 & 0,39 & & \\
\hline 0,14 & 0,18 & 0,18 & 0,19 & 0,17 & \\
\hline & & & & & 0,01 \\
\hline 10 ohne tape & & & & & \\
\hline 0,39 & 0,37 & 0,45 & 0,43 & & \\
\hline 0,19 & 0,17 & 0,25 & 0,23 & 0,21 & \\
\hline 10 mit tape & & & & & \\
\hline 0,45 & 0,44 & 0,47 & 0,48 & & \\
\hline 0,25 & 0,24 & 0,27 & 0,28 & 0,26 & \\
\hline & & & & & 0,05 \\
\hline 11 ohne tape & & & & & \\
\hline 0,36 & 0,37 & 0,39 & 0,38 & & \\
\hline 0,16 & 0,17 & 0,19 & 0,18 & 0,17 & \\
\hline 11 mit tape & & & & & \\
\hline 0,40 & 0,45 & 0,45 & 0,44 & & \\
\hline 0,19 & 0,25 & 0,25 & 0,24 & 0,23 & \\
\hline & & & & & 0,06 \\
\hline
\end{tabular}




\begin{tabular}{|c|c|c|c|c|c|}
\hline 12 ohne tape & & & & & \\
\hline 0,34 & 0,39 & 0,36 & 0,37 & & \\
\hline 0,14 & 0,19 & 0,16 & 0,17 & 0,16 & \\
\hline 12 mit tape & & & & & \\
\hline 0,40 & 0,41 & 0,38 & 0,39 & & \\
\hline 0,20 & 0,21 & 0,18 & 0,19 & 0,19 & \\
\hline & & & & & 0,03 \\
\hline 13 ohne tape & & & & & \\
\hline 0,44 & 0,43 & 0,49 & 0,50 & & \\
\hline 0,24 & 0,23 & 0,29 & 0,31 & 0,27 & \\
\hline 13 mit tape & & & & & \\
\hline 0,49 & 0,51 & 0,50 & 0,49 & & \\
\hline 0,29 & 0,32 & 0,31 & 0,29 & 0,30 & \\
\hline & & & & & 0,04 \\
\hline 14 ohne tape & & & & & \\
\hline 0,48 & 0,47 & 0,49 & 0,49 & & \\
\hline 0,28 & 0,27 & 0,29 & 0,29 & 0,29 & \\
\hline 14 mit tape & & & & & \\
\hline 0,42 & 0,41 & 0,45 & 0,44 & & \\
\hline 0,22 & 0,21 & 0,25 & 0,24 & 0,23 & \\
\hline & & & & & $-0,06$ \\
\hline 15 ohne tape & & & & & \\
\hline 0,30 & 0,38 & 0,32 & 0,36 & & \\
\hline 0,11 & 0,18 & 0,13 & 0,16 & 0,14 & \\
\hline 15 mit tape & & & & & \\
\hline 0,40 & 0,41 & 0,45 & 0,43 & & \\
\hline 0,19 & 0,21 & 0,25 & 0,23 & 0,22 & \\
\hline & & & & & 0,08 \\
\hline 16 ohne tape & & & & & \\
\hline 0,42 & 0,44 & 0,46 & 0,44 & & \\
\hline 0,22 & 0,24 & 0,26 & 0,24 & 0,24 & \\
\hline 16 mit tape & & & & & \\
\hline 0,36 & 0,38 & 0,40 & 0,44 & & \\
\hline 0,16 & 0,18 & 0,20 & 0,24 & 0,19 & \\
\hline & & & & & $-0,05$ \\
\hline 17 ohne tape & & & & & \\
\hline 0,39 & 0,39 & 0,40 & 0,38 & & \\
\hline 0,19 & 0,19 & 0,20 & 0,18 & 0,19 & \\
\hline 17 mit tape & & & & & \\
\hline 0,37 & 0,36 & 0,39 & 0,37 & & \\
\hline 0,17 & 0,16 & 0,19 & 0,17 & 0,17 & \\
\hline & & & & & $-0,02$ \\
\hline
\end{tabular}




\begin{tabular}{|c|c|c|c|c|c|}
\hline 18 ohne tape & & & & & \\
\hline 0,35 & 0,34 & 0,33 & 0,33 & & \\
\hline 0,15 & 0,14 & 0,13 & 0,13 & 0,14 & \\
\hline 18 mit tape & & & & & \\
\hline 0,35 & 0,35 & 0,31 & 0,31 & & \\
\hline 0,15 & 0,15 & 0,12 & 0,12 & 0,13 & \\
\hline & & & & & $-0,01$ \\
\hline 19 ohne tape & & & & & \\
\hline 0,40 & 0,35 & 0,36 & 0,33 & & \\
\hline 0,20 & 0,15 & 0,16 & 0,13 & 0,16 & \\
\hline 19 mit tape & & & & & \\
\hline 0,40 & 0,45 & 0,47 & 0,43 & & \\
\hline 0,19 & 0,25 & 0,27 & 0,23 & 0,23 & \\
\hline & & & & & 0,07 \\
\hline 20 ohne tape & & & & & \\
\hline 0,45 & 0,47 & 0,45 & 0,48 & & \\
\hline 0,25 & 0,27 & 0,25 & 0,28 & 0,26 & \\
\hline 20 mit tape & & & & & \\
\hline 0,40 & 0,44 & 0,45 & 0,43 & & \\
\hline 0,19 & 0,24 & 0,25 & 0,23 & 0,23 & \\
\hline & & & & & $-0,04$ \\
\hline 21 ohne tape & & & & & \\
\hline 0,48 & 0,48 & 0,49 & 0,48 & & \\
\hline 0,28 & 0,28 & 0,29 & 0,28 & 0,28 & \\
\hline 21 mit tape & & & & & \\
\hline 0,41 & 0,41 & 0,45 & 0,43 & & \\
\hline 0,21 & 0,21 & 0,25 & 0,23 & 0,22 & \\
\hline & & & & & $-0,06$ \\
\hline 22 ohne tape & & & & & \\
\hline 0,34 & 0,36 & 0,40 & 0,39 & & \\
\hline 0,14 & 0,16 & 0,20 & 0,19 & 0,17 & \\
\hline 22 mit tape & & & & & \\
\hline 0,42 & 0,41 & 0,45 & 0,43 & & \\
\hline 0,22 & 0,21 & 0,25 & 0,23 & 0,22 & \\
\hline & & & & & 0,05 \\
\hline 23 ohne tape & & & & & \\
\hline 0,40 & 0,42 & 0,45 & 0,45 & & \\
\hline 0,20 & 0,22 & 0,25 & 0,25 & 0,23 & \\
\hline 23 mit tape & & & & & \\
\hline 0,40 & 0,41 & 0,45 & 0,43 & & \\
\hline 0,19 & 0,21 & 0,25 & 0,23 & 0,22 & \\
\hline & & & & & $-0,01$ \\
\hline
\end{tabular}




\begin{tabular}{|c|c|c|c|c|c|}
\hline 24 ohne tape & & & & & \\
\hline 0,36 & 0,33 & 0,43 & 0,40 & & \\
\hline 0,16 & 0,13 & 0,23 & 0,20 & 0,18 & \\
\hline 24 mit tape & & & & & \\
\hline 0,40 & 0,41 & 0,45 & 0,43 & & \\
\hline 0,19 & 0,21 & 0,25 & 0,23 & 0,22 & \\
\hline & & & & & 0,04 \\
\hline 25 ohne tape & & & & & \\
\hline 0,55 & 0,52 & 0,52 & 0,56 & & \\
\hline 0,37 & 0,33 & 0,33 & 0,38 & 0,35 & \\
\hline 25 mit tape & & & & & \\
\hline 0,50 & 0,53 & 0,50 & 0,51 & & \\
\hline 0,31 & 0,34 & 0,31 & 0,32 & 0,32 & \\
\hline & & & & & $-0,04$ \\
\hline 26 ohne tape & & & & & \\
\hline 0,34 & 0,38 & 0,40 & 0,43 & & \\
\hline 0,14 & 0,18 & 0,20 & 0,23 & 0,19 & \\
\hline 26 mit tape & & & & & \\
\hline 0,42 & 0,41 & 0,44 & 0,43 & & \\
\hline 0,22 & 0,21 & 0,24 & 0,23 & 0,22 & \\
\hline & & & & & 0,04 \\
\hline 27 ohne tape & & & & & \\
\hline 0,48 & 0,47 & 0,52 & 0,48 & & \\
\hline 0,28 & 0,27 & 0,33 & 0,28 & 0,29 & \\
\hline 27 mit tape & & & & & \\
\hline 0,45 & 0,42 & 0,45 & 0,43 & & \\
\hline 0,25 & 0,22 & 0,25 & 0,23 & 0,24 & \\
\hline & & & & & $-0,06$ \\
\hline 28 ohne tape & & & & & \\
\hline 0,38 & 0,38 & 0,39 & 0,40 & & \\
\hline 0,18 & 0,18 & 0,19 & 0,20 & 0,18 & \\
\hline 28 mit tape & & & & & \\
\hline 0,45 & 0,50 & 0,45 & 0,46 & & \\
\hline 0,25 & 0,31 & 0,25 & 0,26 & 0,27 & \\
\hline & & & & & 0,08 \\
\hline 29 ohne tape & & & & & \\
\hline 0,38 & 0,39 & 0,42 & 0,39 & & \\
\hline 0,18 & 0,19 & 0,22 & 0,19 & 0,19 & \\
\hline 29 mit tape & & & & & \\
\hline 0,40 & 0,46 & 0,45 & 0,45 & & \\
\hline 0,19 & 0,26 & 0,25 & 0,25 & 0,24 & \\
\hline & & & & & 0,05 \\
\hline
\end{tabular}




\begin{tabular}{|c|c|c|c|c|c|}
\hline 30 ohne tape & & & & & \\
\hline 0,33 & 0,33 & 0,39 & 0,44 & & \\
\hline 0,13 & 0,13 & 0,19 & 0,24 & 0,17 & \\
\hline 30 mit tape & & & & & \\
\hline 0,44 & 0,41 & 0,38 & 0,43 & & \\
\hline 0,24 & 0,21 & 0,18 & 0,23 & 0,21 & \\
\hline & & & & & 0,04 \\
\hline 31 ohne tape & & & & & \\
\hline 0,36 & 0,42 & 0,33 & 0,48 & & \\
\hline 0,16 & 0,22 & 0,13 & 0,28 & 0,20 & \\
\hline 31 mit tape & & & & & \\
\hline 0,47 & 0,48 & 0,45 & 0,43 & & \\
\hline 0,27 & 0,28 & 0,25 & 0,23 & 0,26 & \\
\hline & & & & & 0,06 \\
\hline 32 ohne tape & & & & & \\
\hline 0,30 & 0,32 & 0,42 & 0,46 & & \\
\hline 0,11 & 0,13 & 0,22 & 0,26 & 0,18 & \\
\hline 32 mit tape & & & & & \\
\hline 0,40 & 0,45 & 0,45 & 0,43 & & \\
\hline 0,19 & 0,25 & 0,25 & 0,23 & 0,23 & \\
\hline & & & & & 0,05 \\
\hline 33 ohne tape & & & & & \\
\hline 0,31 & 0,33 & 0,39 & 0,40 & & \\
\hline 0,12 & 0,13 & 0,19 & 0,20 & 0,16 & \\
\hline 33 mit tape & & & & & \\
\hline 0,40 & 0,40 & 0,45 & 0,43 & & \\
\hline 0,19 & 0,20 & 0,25 & 0,23 & 0,22 & \\
\hline & & & & & 0,06 \\
\hline 34 ohne tape & & & & & \\
\hline 0,38 & 0,33 & 0,40 & 0,37 & & \\
\hline 0,18 & 0,13 & 0,20 & 0,17 & 0,17 & \\
\hline 34 mit tape & & & & & \\
\hline 0,40 & 0,41 & 0,45 & 0,42 & & \\
\hline 0,19 & 0,21 & 0,25 & 0,22 & 0,22 & \\
\hline & & & & & 0,05 \\
\hline 35 ohne tape & & & & & \\
\hline 0,39 & 0,42 & 0,43 & 0,42 & & \\
\hline 0,19 & 0,22 & 0,23 & 0,22 & 0,21 & \\
\hline 35 mit tape & & & & & \\
\hline 0,45 & 0,43 & 0,45 & 0,43 & & \\
\hline 0,25 & 0,23 & 0,25 & 0,23 & 0,24 & \\
\hline & & & & & 0,03 \\
\hline
\end{tabular}




\section{E - ROM flex/ex beidbein}

\begin{tabular}{|c|c|c|c|c|c|c|c|c|c|c|c|c|c|c|c|c|c|c|}
\hline \multirow[t]{2}{*}{ bdb. } & & \multicolumn{3}{|c|}{ vor Bel. } & \multicolumn{3}{|c|}{$\mathrm{J} 1$} & \multicolumn{3}{|c|}{32} & \multicolumn{3}{|c|}{33} & \multicolumn{3}{|c|}{34} \\
\hline & flex & & ex & flex & & ex & flex & & ex & flex & & ex & flex & & ex & flex & & ex \\
\hline & 22 & 0 & 68 & 11 & 0 & 47 & 13 & 0 & 52 & 15 & 0 & 55 & 15 & 0 & 56 & 16 & 0 & 56 \\
\hline 1 & 20 & 0 & 75 & 10 & 0 & 55 & 10 & 0 & 65 & 15 & 0 & 65 & 15 & 0 & 65 & 15 & 0 & 65 \\
\hline 2 & 30 & 0 & 55 & 10 & 0 & 45 & 15 & 0 & 50 & 20 & & 50 & 20 & 0 & 50 & 20 & 0 & 50 \\
\hline 3 & 30 & 0 & 60 & 15 & 0 & 45 & 15 & 0 & 50 & 15 & & 60 & 20 & 0 & 60 & 20 & 0 & 60 \\
\hline 4 & 20 & 0 & 50 & 10 & 0 & 40 & 10 & 0 & 50 & 10 & & 50 & 10 & & 50 & 10 & 0 & 50 \\
\hline 5 & 25 & 0 & 75 & 10 & 0 & 45 & 15 & 0 & 50 & 20 & & 55 & 20 & 0 & 55 & 20 & 0 & 65 \\
\hline 6 & 20 & 0 & 80 & 15 & 0 & 40 & 15 & 0 & 50 & 15 & & 55 & 15 & 0 & 60 & 15 & 0 & 60 \\
\hline 7 & 15 & 0 & 90 & 10 & 0 & 45 & 10 & 0 & 55 & 10 & 0 & 60 & 10 & 0 & 65 & 10 & 0 & 65 \\
\hline 8 & 15 & 0 & 65 & 15 & 0 & 40 & 5 & 0 & 40 & 10 & 0 & 50 & 10 & 0 & 50 & 10 & 0 & 50 \\
\hline 9 & 20 & 0 & 80 & 10 & 0 & 55 & 10 & & 65 & 10 & & 70 & 15 & 0 & 70 & 15 & 0 & 70 \\
\hline 10 & 0 & 0 & 70 & 0 & 0 & 55 & 0 & 0 & 60 & 0 & & 60 & 0 & 0 & 60 & 0 & 0 & 60 \\
\hline 11 & 30 & 0 & 70 & 20 & 0 & 45 & 20 & 0 & 45 & 25 & 0 & 55 & 25 & 0 & 55 & 25 & 0 & 55 \\
\hline 12 & 35 & 0 & 70 & 20 & 0 & 40 & 25 & 0 & 55 & 25 & 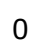 & 55 & 25 & 0 & 55 & 25 & 0 & 55 \\
\hline 13 & 25 & 0 & 75 & 15 & 0 & 55 & 15 & 0 & 60 & 15 & 0 & 60 & 15 & 0 & 60 & 15 & 0 & 60 \\
\hline 14 & 25 & 0 & 55 & 20 & 0 & 40 & 20 & 0 & 40 & 20 & U & 40 & 20 & 0 & 40 & 20 & 0 & 40 \\
\hline 15 & 20 & 0 & 60 & 10 & 0 & 40 & 15 & 0 & 40 & 15 & 0 & 45 & 15 & 0 & 45 & 15 & 0 & 45 \\
\hline 16 & 30 & 0 & 90 & 10 & 0 & 45 & 10 & 0 & 50 & 10 & 0 & 60 & 15 & 0 & 65 & 15 & 0 & 65 \\
\hline 17 & 15 & 0 & 50 & 5 & 0 & 45 & 15 & 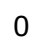 & 45 & 15 & O & 45 & 15 & 0 & 45 & 15 & 0 & 45 \\
\hline 18 & 25 & 0 & 80 & 10 & 0 & 60 & 20 & & 70 & 20 & & 70 & 20 & 0 & 70 & 20 & 0 & 70 \\
\hline 19 & 25 & 0 & 75 & 5 & 0 & 55 & 5 & 0 & 55 & 10 & 0 & 55 & 10 & 0 & 60 & 15 & 0 & 60 \\
\hline 20 & 20 & 0 & 40 & 15 & 0 & 30 & 15 & & 30 & 15 & 0 & 30 & 15 & 0 & 30 & 15 & 0 & 30 \\
\hline 21 & 25 & 0 & 65 & 10 & 0 & 50 & 10 & 0 & 50 & 10 & 0 & 55 & 15 & 0 & 55 & 15 & 0 & 55 \\
\hline 22 & 30 & 0 & 60 & 20 & 0 & 45 & 20 & 0 & 45 & 25 & 0 & 45 & 25 & 0 & 45 & 25 & 0 & 45 \\
\hline 23 & 30 & 0 & 60 & 10 & 0 & 45 & 10 & 0 & 45 & 20 & 0 & 45 & 20 & 0 & 45 & 20 & 0 & 45 \\
\hline 24 & 15 & 0 & 90 & 5 & 0 & 50 & 10 & & 50 & 10 & 0 & 65 & 10 & 0 & 65 & 10 & 0 & 65 \\
\hline 25 & 15 & 0 & 85 & 15 & 0 & 65 & 15 & 0 & 65 & 15 & 0 & 65 & 15 & 0 & 65 & 15 & 0 & 65 \\
\hline 26 & 10 & 0 & 55 & 10 & 0 & 45 & 10 & , & 45 & 10 & 0 & 45 & 10 & 0 & 45 & 10 & 0 & 45 \\
\hline 27 & 0 & 0 & 80 & 0 & 0 & 50 & 0 & 0 & 65 & 0 & 0 & 70 & 0 & 0 & 70 & 0 & 0 & 70 \\
\hline 28 & 15 & 0 & 60 & 10 & 0 & 45 & 10 & 0 & 60 & 10 & 0 & 60 & 10 & 0 & 60 & 10 & 0 & 60 \\
\hline 29 & 30 & 0 & 55 & 10 & 0 & 40 & 10 & 0 & 40 & 20 & 0 & 40 & 20 & 0 & 40 & 20 & 0 & 40 \\
\hline 30 & 0 & 0 & 55 & 0 & 0 & 45 & 0 & 0 & 45 & 0 & 0 & 45 & 0 & 0 & 45 & 0 & 0 & 45 \\
\hline 31 & 20 & 0 & 60 & 10 & 0 & 45 & 10 & 0 & 45 & 15 & 0 & 50 & 15 & 0 & 50 & 15 & 0 & 50 \\
\hline 32 & 20 & 0 & 45 & 15 & 0 & 40 & 15 & 0 & 45 & 15 & 0 & 45 & 15 & 0 & 45 & 15 & 0 & 45 \\
\hline 33 & 30 & 0 & 80 & 15 & 0 & 60 & 20 & 0 & 70 & 20 & 0 & 70 & 20 & 0 & 70 & 20 & 0 & 70 \\
\hline 34 & 35 & 0 & 75 & 20 & 0 & 50 & 20 & 0 & 55 & 25 & 0 & 60 & 25 & 0 & 60 & 25 & 0 & 65 \\
\hline 35 & 40 & 0 & 90 & 15 & 0 & 55 & 30 & 0 & 65 & 30 & 0 & 70 & 30 & 0 & 75 & 30 & 0 & 75 \\
\hline
\end{tabular}




\section{F - ROM sup/pro bdb}

\begin{tabular}{|c|c|c|c|c|c|c|c|c|c|c|c|c|c|c|c|c|c|c|}
\hline \multirow[t]{2}{*}{ bdb. } & & $\begin{array}{c}\text { no } \\
\mathrm{T}\end{array}$ & \multicolumn{4}{|c|}{ vor Bel. } & \multicolumn{3}{|c|}{ J1 } & & & \\
\hline & Sup & & Pro & Sup & & Pro & Sup & & Pro & Sup & & Pro & Sup & & Pro & Sup & & Pro \\
\hline & 49 & 0 & 12 & 14 & 0 & 5 & 15 & 0 & 5 & 16 & 0 & 6 & 18 & 0 & 6 & 19 & 0 & 6 \\
\hline 1 & 40 & 0 & 15 & 10 & 0 & 5 & 10 & 0 & 5 & 15 & 0 & 5 & 15 & 0 & 5 & 15 & 0 & 5 \\
\hline 2 & 60 & 0 & 10 & 15 & 0 & 5 & 20 & 0 & 10 & 20 & 0 & 10 & 30 & 0 & 10 & 30 & 0 & 10 \\
\hline 3 & 40 & 0 & 20 & 20 & 0 & 5 & 20 & 0 & 5 & 20 & 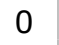 & 5 & 20 & 0 & 5 & 20 & & 5 \\
\hline 4 & 45 & 0 & 15 & 15 & 0 & 0 & 15 & 0 & 0 & 15 & 0 & 0 & 15 & 0 & 0 & 15 & & 0 \\
\hline 5 & 50 & 0 & 10 & 10 & 0 & 5 & 10 & 0 & 5 & 10 & 0 & 5 & 20 & 0 & 5 & 20 & & 5 \\
\hline 6 & 45 & 0 & 15 & 10 & 0 & 5 & 25 & 0 & 10 & 25 & o & 10 & 25 & 0 & 10 & 25 & & 10 \\
\hline 7 & 50 & 0 & 20 & 15 & 0 & 10 & 15 & 0 & 10 & 15 & $c$ & 10 & 15 & 0 & 10 & 15 & & 15 \\
\hline 8 & 50 & 0 & 15 & 15 & 0 & 5 & 15 & 0 & 5 & 20 & & 10 & 20 & 0 & 10 & 20 & & 10 \\
\hline 9 & 60 & 0 & 5 & 10 & 0 & 5 & 10 & 0 & 5 & 15 & ( & & 15 & 0 & 5 & 20 & & 5 \\
\hline 10 & 65 & 0 & 20 & 20 & 0 & 10 & 20 & 0 & 10 & 20 & 0 & 10 & 25 & 0 & 10 & 25 & & 15 \\
\hline 11 & 55 & 0 & 0 & 15 & 0 & 0 & 15 & 0 & 0 & 15 & & 0 & 15 & 0 & 0 & 15 & & 0 \\
\hline 12 & 50 & 0 & 10 & 10 & 0 & 5 & 15 & 0 & 5 & 15 & & 5 & 20 & & 5 & 20 & & 5 \\
\hline 13 & 55 & 0 & 10 & 15 & 0 & 5 & 15 & 0 & 5 & 15 & 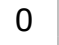 & 5 & 15 & v & 5 & 15 & & 5 \\
\hline 14 & 45 & 0 & 5 & 10 & 0 & 0 & 15 & 0 & 0 & 15 & 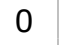 & 0 & 15 & 0 & 0 & 15 & & 0 \\
\hline 15 & 45 & 0 & 15 & 10 & 0 & 5 & 15 & 0 & 5 & 15 & c & 10 & 15 & 0 & 10 & 15 & & 10 \\
\hline 16 & 40 & 0 & 20 & 20 & 0 & 10 & 20 & 0 & 10 & 20 & & 5 & 20 & 0 & 10 & 20 & & 10 \\
\hline 17 & 60 & 0 & 5 & 10 & 0 & 5 & 15 & 0 & 5 & 20 & 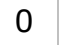 & 5 & 25 & U & 5 & 25 & & 5 \\
\hline 18 & 50 & 0 & 10 & 20 & 0 & 5 & 20 & 0 & 5 & 20 & 0 & 10 & 20 & & 5 & 20 & & 5 \\
\hline 19 & 55 & 0 & 20 & 15 & 0 & 10 & 15 & 0 & 10 & 15 & $c$ & 10 & 15 & 0 & 10 & 15 & ( & 10 \\
\hline 20 & 55 & 0 & 15 & 15 & 0 & 5 & 15 & 0 & 10 & 15 & 0 & & 15 & 0 & 10 & 15 & c & 10 \\
\hline 21 & 45 & 0 & 10 & 15 & 0 & 0 & 15 & 0 & 0 & 15 & & 5 & 15 & 0 & 0 & 15 & & 0 \\
\hline 22 & 50 & 0 & 10 & 20 & 0 & 5 & 25 & 0 & 5 & 25 & $c$ & 5 & 30 & U & 5 & 30 & & 5 \\
\hline 23 & 40 & 0 & 15 & 15 & 0 & 0 & 15 & 0 & 0 & 15 & 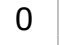 & 0 & 15 & 0 & 5 & 15 & & 5 \\
\hline 24 & 40 & 0 & 5 & 10 & 0 & 0 & 10 & 0 & 0 & 10 & ( & 5 & 10 & 0 & 0 & 10 & & 0 \\
\hline 2 & 35 & 0 & 15 & 10 & 0 & 5 & 10 & $c$ & 5 & 10 & & & 15 & & 5 & 15 & & 5 \\
\hline 26 & 40 & 0 & 10 & 15 & 0 & 5 & 15 & 0 & 5 & 15 & 0 & 10 & 20 & 0 & 5 & 20 & 0 & 5 \\
\hline 27 & 60 & 0 & 5 & 15 & 0 & 5 & 15 & 0 & 5 & 15 & 0 & 5 & 25 & 0 & 5 & 25 & 0 & 5 \\
\hline 28 & 55 & 0 & 10 & 10 & 0 & 5 & 20 & 0 & 5 & 20 & 0 & 5 & 20 & 0 & 5 & 20 & c & 5 \\
\hline 29 & 55 & 0 & 10 & 10 & 0 & 0 & 10 & 4 & 0 & 10 & 4 & 0 & 10 & 0 & 0 & 10 & & 0 \\
\hline 3 & 45 & 0 & 5 & 15 & 0 & 0 & 15 & 0 & 0 & 15 & $c$ & 0 & 15 & 0 & 0 & 15 & & 0 \\
\hline 31 & 50 & 0 & 10 & 15 & 0 & 5 & 20 & 0 & 5 & 20 & 0 & 5 & 20 & 0 & 5 & 20 & 0 & 5 \\
\hline 32 & 50 & 0 & 15 & 20 & 0 & 10 & 20 & 0 & 10 & 25 & 0 & 10 & 25 & 0 & 10 & 25 & 0 & 10 \\
\hline 33 & 40 & 0 & 20 & 10 & 0 & 10 & 10 & 0 & 10 & 10 & 0 & 15 & 15 & 0 & 15 & 15 & 0 & 15 \\
\hline 3 & 45 & 0 & 20 & 5 & 0 & 10 & 5 & 0 & 10 & 15 & U & 10 & 15 & 0 & 10 & 15 & 0 & 10 \\
\hline & 45 & 0 & 10 & 15 & 0 & 5 & 15 & U & & 15 & & & 15 & & & 20 & 0 & \\
\hline
\end{tabular}




\section{G - ROM flex/ex einbn}

\begin{tabular}{|c|c|c|c|c|c|c|c|c|c|c|c|c|c|c|c|c|c|c|}
\hline \multirow{3}{*}{$35^{\circ}$} & \multirow{2}{*}{\multicolumn{3}{|c|}{$\begin{array}{c}\text { Ohne } \\
T\end{array}$}} & \multirow{2}{*}{\multicolumn{3}{|c|}{ Vor Bel. }} & \multirow{2}{*}{\multicolumn{3}{|c|}{$\mathrm{J} 1$}} & \multirow{2}{*}{\multicolumn{3}{|c|}{32}} & \multirow{2}{*}{\multicolumn{3}{|c|}{$\mathrm{J} 3$}} & \multirow{2}{*}{\multicolumn{3}{|c|}{34}} \\
\hline & & & & & & & & & & & & & & & & & & \\
\hline & Flex & & Ext & Flex & & Ext & Flex & & Ext & Flex & & Ext & Flex & & Ext & Flex & & Ext \\
\hline & 22 & 0 & 68 & 11 & 0 & 48 & 13 & 0 & 53 & 15 & 0 & 55 & 15 & 0 & 56 & 16 & 0 & 56 \\
\hline 1 & 20 & 0 & 75 & 10 & 0 & 55 & 10 & 0 & 65 & 15 & 0 & 65 & 15 & 0 & 65 & 15 & & 65 \\
\hline 2 & 30 & 0 & 55 & 10 & 0 & 40 & 15 & 0 & 50 & 20 & 0 & 50 & 20 & 0 & 50 & 20 & 0 & 50 \\
\hline 3 & 30 & 0 & 60 & 15 & 0 & 45 & 15 & 0 & 50 & 15 & 0 & 60 & 20 & 0 & 60 & 20 & & 60 \\
\hline 4 & 20 & 0 & 50 & 10 & 0 & 40 & 10 & 0 & 50 & 10 & 0 & 50 & 10 & 0 & 50 & 10 & 0 & 50 \\
\hline 5 & 25 & 0 & 60 & 10 & 0 & 40 & 15 & 0 & 40 & 20 & 0 & 45 & 20 & 0 & 45 & 20 & 0 & 45 \\
\hline 6 & 20 & 0 & 90 & 15 & 0 & 50 & 15 & 0 & 50 & 15 & 0 & 65 & 15 & 0 & 65 & 15 & 0 & 65 \\
\hline 7 & 15 & 0 & 50 & 10 & 0 & 45 & 10 & 0 & 45 & 10 & 0 & 45 & 10 & 0 & 45 & 10 & C & 45 \\
\hline 8 & 15 & 0 & 80 & 15 & 0 & 60 & 5 & 0 & 70 & 10 & 0 & 70 & 10 & 0 & 70 & 10 & 0 & 70 \\
\hline 9 & 20 & 0 & 75 & 10 & 0 & 55 & 10 & 0 & 60 & 10 & 0 & 55 & 15 & 0 & 60 & 15 & 0 & 60 \\
\hline 10 & 0 & 0 & 40 & 0 & 0 & 30 & 0 & 0 & 30 & 0 & 0 & 30 & 0 & 0 & 30 & 0 & 0 & 30 \\
\hline 11 & 30 & 0 & 65 & 20 & 0 & 50 & 20 & 0 & 50 & 25 & 0 & 55 & 25 & 0 & 55 & 25 & U & 55 \\
\hline 12 & 35 & 0 & 70 & 20 & 0 & 50 & 25 & 0 & 60 & 25 & 0 & 55 & 25 & 0 & 55 & 25 & 0 & 55 \\
\hline 13 & 25 & 0 & 75 & 15 & 0 & 55 & 15 & 0 & 60 & 15 & 0 & 60 & 15 & 0 & 60 & 15 & v & 60 \\
\hline 14 & 25 & 0 & 55 & 20 & 0 & 40 & 20 & 0 & 40 & 20 & 0 & 40 & 20 & 0 & 40 & 20 & 0 & 40 \\
\hline 15 & 20 & 0 & 75 & 10 & 0 & 45 & 15 & 0 & 55 & 15 & 0 & 55 & 15 & 0 & 55 & 15 & & 65 \\
\hline 16 & 30 & 0 & 80 & 10 & 0 & 40 & 10 & 0 & 50 & 10 & 0 & 55 & 15 & 0 & 60 & 15 & 0 & 60 \\
\hline 17 & 15 & 0 & 90 & 5 & 0 & 45 & 15 & 0 & 55 & 15 & 0 & 65 & 15 & 0 & 65 & 15 & & 65 \\
\hline 18 & 25 & 0 & 65 & 10 & 0 & 40 & 20 & 0 & 45 & 20 & 0 & 50 & 20 & 0 & 50 & 20 & 0 & 50 \\
\hline 19 & 25 & 0 & 80 & 5 & 0 & 55 & 5 & 0 & 65 & 10 & 0 & 70 & 10 & 0 & 70 & 15 & 0 & 70 \\
\hline 20 & 20 & 0 & 70 & 15 & 0 & 55 & 15 & 0 & 60 & 15 & 0 & 60 & 15 & 0 & 60 & 15 & 0 & 60 \\
\hline 21 & 25 & 0 & 70 & 10 & 0 & 45 & 10 & 0 & 45 & 10 & 0 & 55 & 15 & 0 & 55 & 15 & & 55 \\
\hline 22 & 30 & 0 & 60 & 20 & 0 & 45 & 20 & 0 & 45 & 25 & 0 & 45 & 25 & 0 & 45 & 25 & 0 & 45 \\
\hline 23 & 30 & 0 & 60 & 10 & 0 & 45 & 10 & 0 & 45 & 20 & 0 & 45 & 20 & 0 & 45 & 20 & $U$ & 45 \\
\hline 24 & 15 & 0 & 90 & 5 & 0 & 50 & 10 & 0 & 60 & 10 & 0 & 65 & 10 & 0 & 65 & 10 & U & 65 \\
\hline 25 & 15 & 0 & 85 & 15 & 0 & 65 & 15 & 0 & 65 & 15 & 0 & 65 & 15 & 0 & 65 & 15 & 0 & 65 \\
\hline 26 & 10 & 0 & 55 & 10 & 0 & 45 & 10 & 0 & 50 & 10 & 0 & 45 & 10 & 0 & 45 & 10 & 0 & 45 \\
\hline 27 & 0 & 0 & 80 & 0 & 0 & 55 & 0 & 0 & 65 & 0 & 0 & 70 & 0 & 0 & 70 & 0 & 0 & 70 \\
\hline 28 & 15 & 0 & 60 & 10 & 0 & 45 & 10 & 0 & 60 & 10 & 0 & 60 & 10 & 0 & 60 & 10 & 0 & 60 \\
\hline 29 & 30 & 0 & 55 & 10 & 0 & 40 & 10 & 0 & 40 & 20 & 0 & 40 & 20 & 0 & 40 & 20 & 0 & 40 \\
\hline 30 & 0 & 0 & 55 & 0 & 0 & 45 & 0 & 0 & 45 & 0 & 0 & 45 & 0 & 0 & 45 & 0 & 0 & 45 \\
\hline 31 & 20 & 0 & 60 & 10 & 0 & 45 & 10 & 0 & 50 & 15 & 0 & 50 & 15 & 0 & 50 & 15 & 0 & 50 \\
\hline 32 & 20 & 0 & 45 & 15 & 0 & 40 & 15 & 0 & 45 & 15 & 0 & 45 & 15 & 0 & 45 & 15 & 0 & 45 \\
\hline 33 & 30 & 0 & 80 & 15 & 0 & 60 & 20 & 0 & 70 & 20 & 0 & 70 & 20 & 0 & 70 & 20 & 0 & 70 \\
\hline 34 & 35 & 0 & 75 & 20 & 0 & 50 & 20 & 0 & 55 & 25 & 0 & 60 & 25 & 0 & 60 & 25 & 0 & 65 \\
\hline 35 & 40 & 0 & 90 & 15 & 0 & 55 & 30 & 0 & 70 & 30 & 0 & 70 & 30 & 0 & 75 & 30 & 0 & 75 \\
\hline
\end{tabular}




\section{H- ROM sup/pro einbn}

\begin{tabular}{|c|c|c|c|c|c|c|c|c|c|c|c|c|c|c|c|c|c|c|}
\hline & \multicolumn{3}{|c|}{$\begin{array}{c}\text { Ohne } \\
\mathrm{T}\end{array}$} & \multicolumn{3}{|c|}{ Vor Bel. } & \multicolumn{3}{|c|}{$\mathrm{J} 1$} & \multicolumn{3}{|c|}{$\mathrm{J} 2$} & \multicolumn{3}{|c|}{ J3 } & \multicolumn{3}{|c|}{34} \\
\hline & Sup & & Pro & Sup & & Pro & Sup & & Pro & Sup & & Pro & Sup & & Pro & Sup & & Pro \\
\hline & 49 & 0 & 12 & 14 & 0 & 5 & 19 & 0 & 6 & 26 & 0 & 7 & 29 & 0 & 7 & 35 & 0 & 8 \\
\hline 1 & 40 & 0 & 15 & 10 & 0 & 5 & 15 & 0 & 5 & 25 & 0 & 10 & 25 & 0 & 10 & 25 & 0 & 10 \\
\hline 2 & 60 & 0 & 10 & 15 & 0 & 5 & 20 & 0 & 5 & 25 & 0 & 5 & 25 & 0 & 5 & 45 & 0 & 5 \\
\hline 3 & 40 & 0 & 20 & 20 & 0 & 5 & 25 & 0 & 10 & 30 & 0 & 10 & 35 & 0 & 10 & 35 & 0 & 15 \\
\hline 4 & 45 & 0 & 15 & 15 & 0 & 0 & 25 & 0 & 5 & 20 & 0 & 5 & 25 & 0 & 5 & 30 & 0 & 10 \\
\hline 5 & 50 & 0 & 20 & 10 & 0 & 10 & 30 & U & 15 & 25 & 0 & 15 & 35 & 0 & 15 & 40 & 0 & 15 \\
\hline 6 & 45 & 0 & 5 & 10 & 0 & 5 & 15 & 0 & 5 & 20 & 0 & 5 & 25 & 0 & 5 & 25 & 0 & 5 \\
\hline 7 & 50 & 0 & 10 & 15 & 0 & 5 & 20 & 0 & 5 & 30 & 0 & 5 & 35 & 0 & 5 & 35 & 0 & 5 \\
\hline 8 & 50 & 0 & 20 & 15 & 0 & 10 & 20 & 0 & 10 & 25 & 0 & 15 & 25 & 0 & 15 & 35 & 0 & 15 \\
\hline 9 & 60 & 0 & 15 & 10 & 0 & 5 & 25 & 0 & 5 & 30 & 0 & 10 & 30 & 0 & 10 & 40 & 0 & 10 \\
\hline 10 & 65 & 0 & 10 & 20 & 0 & 0 & 15 & 0 & 5 & 20 & 0 & 5 & 25 & 0 & 5 & 45 & 0 & 5 \\
\hline 11 & 55 & 0 & 10 & 15 & 0 & 5 & 20 & 0 & 5 & 30 & 0 & 5 & 35 & 0 & 5 & 40 & 0 & 5 \\
\hline 12 & 50 & 0 & 15 & 10 & 0 & 0 & 20 & 0 & 5 & 35 & 0 & 5 & 35 & 0 & 5 & 40 & 0 & 10 \\
\hline 13 & 55 & 0 & 5 & 15 & 0 & 0 & 15 & 0 & 0 & 30 & 0 & 5 & 35 & 0 & 5 & 45 & 0 & 5 \\
\hline 14 & 45 & 0 & 15 & 10 & 0 & 5 & 20 & 0 & 15 & 25 & 0 & 15 & 30 & 0 & 15 & 30 & 0 & 15 \\
\hline 15 & 45 & 0 & 10 & 10 & 0 & 5 & 15 & 0 & 5 & 20 & 0 & 5 & 20 & 0 & 5 & 40 & 0 & 5 \\
\hline 16 & 40 & 0 & 10 & 20 & 0 & 5 & 25 & 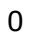 & 5 & 30 & 0 & 5 & 30 & 0 & 5 & 30 & 0 & 5 \\
\hline 17 & 60 & 0 & 15 & 10 & 0 & 5 & 15 & 0 & 0 & 25 & 0 & 0 & 30 & 0 & 0 & 40 & 0 & 10 \\
\hline 18 & 50 & 0 & 20 & 20 & 0 & 10 & 20 & 0 & 10 & 30 & 0 & 10 & 35 & 0 & 15 & 40 & 0 & 15 \\
\hline 19 & 55 & 0 & 15 & 15 & 0 & 5 & 20 & 0 & 5 & 25 & 0 & 10 & 30 & 0 & 10 & 35 & 0 & 10 \\
\hline 20 & 55 & 0 & 5 & 15 & 0 & 5 & 20 & C & 5 & 20 & 0 & 5 & 25 & 0 & 5 & 30 & 0 & 5 \\
\hline 21 & 45 & 0 & 20 & 15 & 0 & 10 & 15 & 0 & 10 & 25 & 0 & 10 & 25 & 0 & 10 & 30 & 0 & 10 \\
\hline 22 & 50 & 0 & 0 & 20 & 0 & 0 & 25 & 0 & 0 & 35 & 0 & 0 & 35 & 0 & 0 & 35 & 0 & 0 \\
\hline 23 & 40 & 0 & 10 & 15 & 0 & 5 & 20 & 0 & 5 & 30 & 0 & 5 & 30 & 0 & 5 & 35 & 0 & 5 \\
\hline 24 & 40 & 0 & 10 & 10 & 0 & 5 & 15 & 0 & 5 & 20 & 0 & 5 & 25 & 0 & 5 & 25 & 0 & 5 \\
\hline 25 & 35 & 0 & 5 & 10 & 0 & 0 & 15 & 0 & 0 & 20 & 0 & 0 & 20 & 0 & 0 & 30 & 0 & 0 \\
\hline 26 & 40 & 0 & 15 & 15 & 0 & 5 & 20 & 0 & 10 & 25 & 0 & 10 & 25 & 0 & 10 & 25 & 0 & 10 \\
\hline 27 & 60 & 0 & 5 & 15 & 0 & 5 & 20 & 0 & 5 & 30 & 0 & 5 & 30 & 0 & 5 & 45 & 0 & 5 \\
\hline 28 & 55 & 0 & 10 & 10 & 0 & 5 & 15 & 0 & 5 & 25 & 0 & 5 & 25 & 0 & 5 & 35 & 0 & 10 \\
\hline 29 & 55 & 0 & 10 & 10 & 0 & 0 & 15 & 0 & 5 & 20 & 0 & 5 & 25 & 0 & 5 & 40 & 0 & 5 \\
\hline 30 & 45 & 0 & 5 & 15 & 0 & 0 & 20 & 0 & 0 & 30 & 0 & 0 & 35 & 0 & 0 & 35 & 0 & 0 \\
\hline 31 & 50 & 0 & 10 & 15 & 0 & 5 & 20 & 0 & 5 & 25 & 0 & 5 & 30 & 0 & 5 & 40 & 0 & 5 \\
\hline 32 & 50 & 0 & 15 & 20 & 0 & 10 & 20 & 0 & 10 & 25 & 0 & 10 & 25 & 0 & 10 & 40 & 0 & 15 \\
\hline 33 & 40 & 0 & 20 & 10 & 0 & 10 & 15 & 0 & 15 & 30 & 0 & 15 & 35 & 0 & 15 & 35 & 0 & 15 \\
\hline 34 & 45 & 0 & 20 & 5 & 0 & 10 & 15 & 0 & 10 & 35 & 0 & 10 & 35 & 0 & 10 & 35 & 0 & 10 \\
\hline 35 & 45 & 0 & 10 & 15 & 0 & 5 & 25 & 0 & 5 & 30 & 0 & 5 & 30 & 0 & 5 & 30 & 0 & 5 \\
\hline
\end{tabular}




\section{I - ROM flex/ex nach Hochsprung}

\begin{tabular}{|c|c|c|c|c|c|c|c|c|c|c|c|c|c|c|c|c|c|c|c|c|c|}
\hline $\mathrm{HS}$ & & $\begin{array}{l}\text { Ohn } \\
T\end{array}$ & & & $\begin{array}{l}\text { Vo } \\
\text { Bel }\end{array}$ & & & $\mathrm{J} 1$ & & & $\mathrm{~J} 2$ & & & J3 & & & J4 & & & J5 & \\
\hline & Flex & & Ext & Flex & & Ext & Flex & & Ext & Flex & & Ext & Flex & & Ext & Flex & & Ext & Flex & & Ext \\
\hline & 21 & 0 & 68 & 12 & 0 & 48 & 13 & 0 & 55 & 13 & 0 & 56 & 15 & 0 & 57 & 15 & 0 & 58 & 15 & 0 & 58 \\
\hline 1 & 30 & 0 & 60 & 15 & 0 & 45 & 15 & 0 & 50 & 15 & 0 & 60 & 20 & 0 & 60 & 20 & 0 & 60 & 20 & 0 & 60 \\
\hline 2 & 20 & 0 & 50 & 10 & 0 & 40 & 10 & 0 & 50 & 10 & 0 & 50 & 10 & 0 & 50 & 10 & 0 & 50 & 10 & 0 & 50 \\
\hline 3 & 20 & 0 & 60 & 10 & 0 & 45 & 10 & 0 & 50 & 15 & 0 & 50 & 15 & 0 & 50 & 15 & 0 & 50 & 15 & 0 & 50 \\
\hline 4 & 20 & 0 & 45 & 15 & 0 & 40 & 15 & 0 & 45 & 15 & 0 & 45 & 15 & 0 & 45 & 15 & 0 & 45 & 15 & 0 & 45 \\
\hline 5 & 10 & 0 & 55 & 10 & 0 & 45 & 10 & 0 & 50 & 10 & 0 & 45 & 10 & 0 & 45 & 10 & 0 & 45 & 10 & 0 & 45 \\
\hline 6 & 5 & 0 & 80 & 0 & 0 & 55 & 0 & 0 & 65 & 0 & 0 & 70 & 0 & 0 & 70 & 0 & 0 & 70 & 5 & 0 & 70 \\
\hline 7 & 20 & 0 & 75 & 10 & 0 & 45 & 15 & 0 & 55 & 15 & 0 & 55 & 15 & 0 & 55 & 15 & 0 & 65 & 15 & 0 & 65 \\
\hline 8 & 30 & 0 & 80 & 10 & 0 & 40 & 10 & 0 & 50 & 10 & 0 & 55 & 15 & 0 & 60 & 15 & 0 & 60 & 15 & 0 & 65 \\
\hline 9 & 25 & 0 & 75 & 15 & 0 & 55 & 15 & 0 & 60 & 15 & 0 & 60 & 15 & 0 & 60 & 15 & 0 & 60 & 15 & 0 & 60 \\
\hline 10 & 25 & 0 & 55 & 20 & 0 & 40 & 20 & 0 & 40 & 20 & 0 & 40 & 20 & 0 & 40 & 20 & 0 & 40 & 20 & 0 & 40 \\
\hline 11 & 15 & 0 & 80 & 15 & 0 & 60 & 5 & 0 & 70 & 10 & 0 & 70 & 10 & 0 & 70 & 10 & 0 & 70 & 10 & 0 & 70 \\
\hline 12 & 20 & 0 & 75 & 10 & 0 & 55 & 10 & 0 & 60 & 10 & 0 & 55 & 15 & 0 & 60 & 15 & 0 & 60 & 15 & 0 & 60 \\
\hline 13 & 20 & 0 & 70 & 15 & 0 & 55 & 15 & 0 & 60 & 15 & 0 & 60 & 15 & 0 & 60 & 15 & 0 & 60 & 15 & 0 & 60 \\
\hline 14 & 25 & 0 & 70 & 10 & 0 & 45 & 10 & 0 & 45 & 10 & 0 & 55 & 15 & 0 & 55 & 15 & 0 & 55 & 15 & 0 & 55 \\
\hline 15 & 35 & 0 & 90 & 15 & 0 & 55 & 30 & 0 & 70 & 30 & 0 & 70 & 30 & 0 & 75 & 30 & 0 & 75 & 30 & 0 & 75 \\
\hline
\end{tabular}




\section{J - ROM sup/pro nach Hochsprung}

\begin{tabular}{|c|c|c|c|c|c|c|c|c|c|c|c|c|c|c|c|c|c|c|c|c|c|}
\hline \multirow[t]{3}{*}{ HS } & \multicolumn{3}{|c|}{$\begin{array}{c}\text { Ohne } \\
T\end{array}$} & \multicolumn{3}{|c|}{$\begin{array}{l}\text { Vor } \\
\text { Bel. }\end{array}$} & \multicolumn{3}{|c|}{$\mathrm{J} 1$} & \multicolumn{3}{|c|}{$\mathrm{J} 2$} & \multicolumn{3}{|c|}{33} & \multicolumn{3}{|c|}{ J4 } & \multicolumn{3}{|c|}{ J5 } \\
\hline & Flex & & Ext & Flex & & Ext & Flex & & Ext & Flex & & Ext & Flex & & Ext & Flex & & Ext & Flex & & Ext \\
\hline & 21 & 0 & 68 & 12 & 0 & 48 & 13 & 0 & 55 & 13 & 0 & 56 & 15 & 0 & 57 & 15 & 0 & 58 & 15 & 0 & 58 \\
\hline 1 & 30 & 0 & 60 & 15 & 0 & 45 & 15 & 0 & 50 & 15 & 0 & 60 & 20 & 0 & 60 & 20 & 0 & 60 & 20 & 0 & 60 \\
\hline 2 & 20 & 0 & 50 & 10 & 0 & 40 & 10 & 0 & 50 & 10 & 0 & 50 & 10 & 0 & 50 & 10 & 0 & 50 & 10 & 0 & 50 \\
\hline 3 & 20 & 0 & 60 & 10 & 0 & 45 & 10 & 0 & 50 & 15 & 0 & 50 & 15 & 0 & 50 & 15 & 0 & 50 & 15 & 0 & 50 \\
\hline 4 & 20 & 0 & 45 & 15 & 0 & 40 & 15 & 0 & 45 & 15 & 0 & 45 & 15 & 0 & 45 & 15 & 0 & 45 & 15 & 0 & 45 \\
\hline 5 & 10 & 0 & 55 & 10 & 0 & 45 & 10 & 0 & 50 & 10 & 0 & 45 & 10 & 0 & 45 & 10 & 0 & 45 & 10 & 0 & 45 \\
\hline 6 & 5 & 0 & 80 & 0 & 0 & 55 & 0 & 0 & 65 & 0 & 0 & 70 & 0 & 0 & 70 & 0 & 0 & 70 & 5 & 0 & 70 \\
\hline 7 & 20 & 0 & 75 & 10 & 0 & 45 & 15 & 0 & 55 & 15 & 0 & 55 & 15 & 0 & 55 & 15 & 0 & 65 & 15 & 0 & 65 \\
\hline 8 & 30 & 0 & 80 & 10 & 0 & 40 & 10 & 0 & 50 & 10 & 0 & 55 & 15 & 0 & 60 & 15 & 0 & 60 & 15 & 0 & 65 \\
\hline 9 & 25 & 0 & 75 & 15 & 0 & 55 & 15 & 0 & 60 & 15 & 0 & 60 & 15 & 0 & 60 & 15 & 0 & 60 & 15 & 0 & 60 \\
\hline 10 & 25 & 0 & 55 & 20 & 0 & 40 & 20 & 0 & 40 & 20 & 0 & 40 & 20 & 0 & 40 & 20 & 0 & 40 & 20 & 0 & 40 \\
\hline 11 & 15 & 0 & 80 & 15 & 0 & 60 & 5 & 0 & 70 & 10 & 0 & 70 & 10 & 0 & 70 & 10 & 0 & 70 & 10 & 0 & 70 \\
\hline 12 & 20 & 0 & 75 & 10 & 0 & 55 & 10 & 0 & 60 & 10 & 0 & 55 & 15 & 0 & 60 & 15 & 0 & 60 & 15 & 0 & 60 \\
\hline 13 & 20 & 0 & 70 & 15 & 0 & 55 & 15 & 0 & 60 & 15 & 0 & 60 & 15 & 0 & 60 & 15 & 0 & 60 & 15 & 0 & 60 \\
\hline 14 & 25 & 0 & 70 & 10 & 0 & 45 & 10 & 0 & 45 & 10 & 0 & 55 & 15 & 0 & 55 & 15 & 0 & 55 & 15 & 0 & 55 \\
\hline 15 & 35 & 0 & 90 & 15 & 0 & 55 & 30 & 0 & 70 & 30 & 0 & 70 & 30 & 0 & 75 & 30 & 0 & 75 & 30 & 0 & 75 \\
\hline
\end{tabular}




\section{Literatur}

1 Allison GT, et al. The influence of rigid taping on peroneal latency in normal ankles. Australian Journal of Physiotherapy 45: 195 - 201. 1999.

2 Alves JW, et al., A comparison of passive support provided by various ankle braces. Journal of Orthop Sports Phys Therapy 15 (1): 10 - 18. 1992

3 Anderson $\mathrm{DL}$, et al. The role of external nonrigid ankle bracing in limiting ankle inversion. Clinical Journal of Sportsmedicine 5: 18 - 24. 1995.

4 Andreasson G, et al., Rheological properties of medical tape used to prevent athletic injuries. Textile Res Journal1983 April; 53.

5 Andry N, Orthopädie, oder die Kunst, bei den Kindern die Ungestaltheit des Leibes zu verhüten und zu verbessern. In: Wessinghage, D. (Hrsg.), Stuttgart 1987, 1744.

6 Balduini FC et al., Management and Rehabilitation of ligamentous injuries to the ankle. Sports Med; 4: 364 - 380; 1987.

7 Bartold SJ, Optoelectronic analysis of support provided by adhesive taping and ankle orthoses before, during and after exercise [abstract]. Annual Scientific Conference in Sports Medicine; 1993 Oct 26 - 31; 324 329; 1932.

8 Bauersfeld, K.H./Schröter, G. Grundlagen der Leichtathletik, Sportverlag, Berlin 1992. 
9 Bauer T, The effectiveness of ankle taping and considerations for alternatives. In: Kreighbaum E et al., editors. $6^{\text {th }}$ International Symposium on Biomechanics in Sports. Bozeman (MT): International Society of Biomechanics in Sports, 1988: 407 - 418.

10 Bennell KL et al., The differential effects of external ankle support on postural control. Journal of Orthop Sport Phys Therapy; 20 (6): 287 295; 1994.

11 Benninghoff - Anatomie, Makroskopische und mikroskopische Anatomie des Menschen. Hrsgb. Fleischhauer K et al., Band 1; Urban \& Schwarzenberg (Elsevier), 1985.

12 Beriau MR et al., Effects of ankle braces upon agility course performance in high school athletes. Journal of Athletic Training 29 (3):224 - 226; 1994.

13 Bladin C et al., Snowboarding injuries: an overview. Sports Med; 19 (5): 358 - 364; 1995.

14 Bocchinfuso $C$ et al., Effects of two semirigid prophylactic ankle stabilizers on speed, agility, and verical jump. Journal of Sport Rehabilitation, 3 (2): 125 - 134; 1994.

15 Bowerman WJ et al., High-Performance Training for Track and Field. Human Kinetics; 1991.

16 Browne $\mathrm{K}$ et al., The sensation of passive movement at the metatarsophalangeal joint of the great toe in man. Journal of Physiology; 374: 335-348; 1954. 
17 Bruns J et al., Mechanical ankle stabilisation due to the use of orthotic devices and peroneal muscle strength. An experimental investigation. International Journal of Sports Medicine; 13 (8): 611 - 615; 1992.

18 Bryan L et al., Effect of Ankle Taping and Bracing on Vertical Ground Reaction Forces During Drop Landings Before and After Treadmill Jogging. Journal of Orthopaedic and Physical Therapy Vol 32. 12: 628 635. 2002.

19 Burks RT, et al. Analysis of athletic performance with prophylactic ankle devices. American Journal of Sportsmedicine; 19(2): 104 - 106; 1991.

20 Chan KM et al., Sports causing most injuries in Hong Kong. British Journal of Sports Medicine; 27 (4): 263-267; 1993.

21 Clark F et al., Proprioception with the proximal interphalangeal joint of the index finger. Brain 109: 1195-1208; 1986.

22 Coffmann JL et al., A comparison of ankle taping and the Aircast Sport Stirrup on athletic performance. Athletic Training; 24 (2): 123; 1989.

23 Conférence de consensus $V$ en médecine d'urgences de la société Francophone d'urgences médicales, Roanne, April 28, Réan. Urgence 4:491 - 555, 1995.

24 Cordova ML et al. Influence of ankle support on joint range of motion before and after exercise: a meta-analysis. Journal of Orthop Sports Phys Therapy 30: 170 - 182. 2000. 
25 Cornwall MW et al. Three-dimensional movement of the foot during the stance phase of walking. Journal of American Podiatric Medical Association 89: 56 - 66. 1999.

26 Dapna, J. (1988). Biomechanical Analysis of the Fosbury Flop. Track Technique. 104, 3307- 3317.

27 Dapena, J. Biomechanical analysis of the Fosbury flop. Track Technique 104:3307-3317;3333; 105:3343-3350, 1988.

28 Dapena, J. How to design the shape of a high jump run-up. Track Coach 131, Spring 1995.

29 Dapena, J. Mechanics of rotation in the Fosbury- flop. Med. Sci. Sports Exerc. 12:45-53, 1980b.

30 Dapena, J. Mechanics of translation in the Fosbury- Flop. Med. Sci. Sports Exerc. 12:37-44, 1980a.

31 Dapena, J. Track and Field High Jump (men and women). 1992 Summer Olympic Games. Biomechanic Projects. Department of Kinesiology, Indiana University, Blumington (Indiana, USA), 1992.

32 Ekstrand J et al., Prevention of soccer injuries: supervision by doctor and physiotherapist. American Journal of Sports Medicine; 11 (3): 116$120 ; 1983$.

33 Ferguson ABJ. The case against ankle taping. Journal of Sports Medicine; $1(2): 46$ - 47; 1973. 
34 Feuerbach J, et al., Effect of an ankle orthosis and ankle ligament anesthesia on ankle joint poprioception. American Journal of Sports Medicine. 22: 223-229, 1994.

35 Firer P. Effectiveness of taping for prevention of ankle ligament sprains. British Journal of Sports Medicine; 24 (1): 47 - 50; 1950.

36 Frankeny JR et al. A comparison of ankle-taping methods. Clin J Sport Med; 3 (1): 20 - 25; 1993.

37 Freeman MA et. al. The aetiology and prevention of functional instability of the foot. Journal of Bone and Joint Surgery 47B: 678 - 685, 1965.

38 Fumich RM et al. Measured effect of taping on combined foot and ankle motion before and after exercise. Am J Sports Med 9 (3): 165 - 170; 1981.

39 Garrick JG et al. Role of external support in the prevention of ankle sprains. Med Sci Sports Exercise 5: 200 - 203. 1973.

40 Gerdle et al., Rank order of peak amplitude of EMG between the three muscles triceps surae during maximal isometric contractions. Scandinavian Journal of Rehabilitation Medicine, 18, 9-15; 1988.

41 Gharavi H, Tape Jobs in $360 \mathrm{sec}$ - Volume II . CD-ROM, Verlag SportsmedSolutions Regensburg; 2004.

42 Glick JM et al., Prevention and treatment of ankle injuries. American Journal of Sportsmedicine; 4(4): 136 - 141, 1976. 
43 Goodwin GM et al., The persistence of appreciable kinesthesia after paralyzing joint afferents but preserving muscle afferents. Brain Research, 38: 326-329. 1972.

$44 \mathrm{Graff}, \mathrm{KH}$. et al., Streßfrakturen des Os naviculare pedis. Zeitschrift $\mathrm{f}$. Orthopädie 1986, 124:228.

45 Greene TA et al., A comparative isokinetic evaluation of a functional ankle orthosis on talocalcaneal function. Journal Orhtop Sport Phys Therapy; 11 (6): 245 - 252; 1989.

46 Greene TA et al., A comparative support evaluation of three ankle orthoses before, during, and after exercise. Journal of Orthop Sport Phys Therapy; 11 (10): 453 - 466; 1990.

47 Grigg, P. (1994) Peripheral neural mechanisms in proprioception. Journal of Sport Rehabilitation 3, 2-17.

48 Gross MT et al., Comparison of support provided by ankle taping and semirigid orthosis. Journal of Orthop Sport Phys Ther; 9 (1): 33 - 39; 1987.

49 Gross MT et al., Comparisons of Donjoy ankle Ligament Protector and subtalar sling ankle taping in restricting foot and ankle motion before and after exercise. Journal of Orthop Sport Phys Therapy, 19 (1): 33 41, 1994. 
50 Gross MT et al., Comparison Swede-O-Universal Ankle Support and Aircast Sport-Stirrup orthoses and ankle tape in resticting eversioninversion before and after exercise. Journal of Orhtop Sport Phys Therapy; 13 (1): 11 - 19; 1991.

51 Gross MT et al., Effect of ankle orthoses on functional performance for individuals with recurrent lateral ankle sprains. Journal of Orthop Sports Phys Ther, 25 (4): 245 - 252; 1997.

52 Gross MT et al., Effect of Donjoy ankle Ligament Protector and Aircast Sport-Stirrup orthoses on functional performance. Journal of Orthop Sports Phys Ther, 19 (3): 150 - 156; 1994.

53 Halseth T, et al. The effects of Kinesio TM Taping on proprioception at the Ankle. Journal of Sports Science and Medicine 2004, 3: 1 - 7.

54 Hals TM, et al. Effect of a semi-rigid ankle stabilizer on performance in persons with functional ankle instability. Journal Orthop Sports Phys Therapy 30: 552 - 556. 2000.

55 Hamill J. Exercise moderation of foot function during walking with a reuseable semirigid ankle orthosis. Clin Biomech; 3: 153 - 158; 1988.

56 Hay, J. Die Hay-Technik. Die Lehre der Leichtathletik. 11.9.1973 (1313 1316), 18.9.1973 (1352).

57 Heit, E., Lephart, S. and Rozzi, S. (1996) The effect of ankle bracing and taping on joint position sense in the stable ankle. Journal of Sport Rehabilitation 5, 206-213; 1996. 
58 Hochman D et al., Alternate methods of ankle stabilization. Journal of Canadian Athletic Therapy Association; 9(1): 11 - 13; 1982.

59 Holmer $\mathrm{P}$ et al., Epidemiology of sprains in the lateral ankle and foot. Foot Ankle Int; 15 (2): 72 - 74; 1994.

60 Hoppenfeld S, Physical Examination of the Spine and Extremities, Appleton \& Lange, 1999.

61 Huang $\mathrm{CH}$, Lace-up ankle brace and athletic tape related to ankle kinesthesia and strength [microfiche]. Eugene (OR): University of Oregon Microform Publications, 1994.

62 Hughes LH et al., A comparison of ankle taping and a semirigid support. Phys Sports Med; 11(4); 99-103; 1983.

63 Hume PA et al., Effectiveness of External Ankle Support - Bracing and Taping in Rugby Union, Sports Med 25 (5): 285 - 312; 1998.

64 Humphrey. S. \& Nordquist. D. (2000). High Jump. In USA Track \& Field Coaching Manual (Joseph Rogers, Editor). Human Kinetics; 2000.

65 Jerosch J et al., Influence of external stabilizing devices of the ankle on sport-specific capabilities. Knee Surg Sports Traumatology Arthrose, 5 (1): 50 - 57; 1997.

66 Jerosch $\mathrm{J}$ et al., Is prophylactic bracing of the ankle cost effective? Orhtopedics 19 (5): 405 - 14; 1996.

67 Jerosch J et al., The influence of orthoses on the proprioceptionof the ankle joint. Knee Surg Sports Traumatol Athrose; 3:39-46; 1995. 
68 Karlsson, J. and Andreasson, G. The effect of external ankle support in chronic lateral ankle joint instability: an electromyographic study. American Journal of Sports Medicine 20, 257-261; 1992.

69 Karlsson J, Chronic lateral instability of the ankle joint. A clinical, radiological and experimental study. Thesis Gothenburg University, Götenborg, Sweden, 1989.

70 Karlsson J et al., Reconstruction of the lateral ligaments of the ankle for chronic lateral instability. Journal of Bone and Joint Surgery - Am Vol 70: 581-588.1988.

71 Karlsson J et al., Surgical treatment of chronic lateral instability of the ankle joint. A new procedure. American Journal of Sports Medicine 17: 268 - 274, 1989.

72 Karlsson J et al., The effect of Taping on Ankle Stability - Practical Implication. Sports Medicine 16 (3): 210-215; 1993.

73 Killing, W. Die Schnelligkeit im Hochsprung. In: Leichathletiktraining 5 (1994a), 1/2, S. $38-48$.

74 Kirst J. Zur Methodik und zu den technischen Fehlern im Anfängertraining der Flopspringer. Leipzig, DhfK, Dipl.Arbeit, S. 45; 1978.

75 KRÜGER A \& OBERDIECK H : Kleiner Ratgeber für Leichtathletikverletzungen. Berlin: Bartels \& Wernitz 1975, pp.112 
76 Kukulka CG et al., Muscle pressure effects on motorneuron reflex excitability: a special communication. Physical Therapy 67: 1720 - 1722. 1987.

77 Laughman RK et al., Three dimensional Kinematics of the taped ankle before and after exercise. American Journal of Sportsmedicine; 8 (6): 425 - 431; 1980.

78 Lentell $\mathrm{G}$ et al., The contributions of proprioceptive deficits, muscle function and anatomical laxity to functional instability of the ankle. J Orthop Sports Phys Ther; 21: 206-215; 1995.

79 Lephart, S., Pincivero, D., Giraldo, J. and Fu, F. (1997) The role of proprioception in the management and rehabilitation of athletic injuries. American Journal of Sports Medicine 25, 130-137.

80 Lindenberger U. Et al., The effect of prophylactic taping of ankles [PhD thesis]. Department of Textile Technology. Chalmers University of Technology, Göteborg, 1985.

81 Locke $A B$, Effect of aoftshell porphylactic ankle stabilizer on performance in events involving speed, agility, and vertical jump during long-term use [thesis]. Eugene OR: Microform Publications, International Institute for Sport \& Human Performance, University of Oregon 1996: I (86)

82 Lohrer H, et al., In: Engelhardt $M$ et al. (Hrsg.) GOTS - Manual der Sporttraumatologie, Verlag Hans-Huber, 473-82, 1997. 
83 Mackean LC et al., Prophylactic ankle bracing vs taping: effects on functional performance in female basketball players. Journal of Orthop Sports Phys Therapy; 22 (2): 11 - 81; 1995.

84 Macpherson K, Effects of asemirigid and a softshell prophylactic ankle stabilizer [microfiche]. Eugene, OR. University of Oregon Microform Publications; 1995.

85 Macpherson $\mathrm{K}$ et al., Effects of a semirigid and softshell prophylactic ankle stabilizer on selected performance tests among high school football players. Journal of Orthop Sports Phys Therapy 21: 147 - 152. 1995.

86 Malina RM et al., Effects of exercise upon measurable supporting strength of cloth and tape ankle wraps. Res Q 34: 158 - 165; 1962.

87 Martin $M$, et al., Comparison of inversion restraint provided by ankle prophylactic devices before and after exercise. Journal of Athletic Training 28 (4): 324 - 329. 1993.

88 Matthews PB, Proprioceptors and their contribution to somatosensory mapping: complex messages require complex processing. Canadian Journal of Physiology and Pharmacology 66: 430-438, 1987.

89 McCloskey DI et al. Role of inputs from skin, joints and muscles and of corollary discharges, in human discriminatory tasks. Aus: Gordon G, ed. Active touch: the mechanism of recognition of objects by manipulation: a multi-disciplinary approach. Oxford: Pergamon Press 1977: 177-88. 
90 McConkey JP. Ankle sprains, consequences and mimics. In: Shephard RJ, Taunton JE, editors. Footand ankle in Sport and Exercise. Basel: Karger, 1987: 39-55.

91 McLean DD, Use of adhesive strapping in sport. Br Journal Sports Med; 23 (3): 147 - 149; 1989.

92 McPoil TG \& Knecht HG (1985) Biomechanics of the foot in walking: A functional approach, Journal of Orthopedic and Sports Physical Therapy, 7, 69-72; 1985.

93 Metcalfe RC, A comparison of moleskin tape, linen tape and lace-up brace on joint restriction and movement performance. Journal of Athletic Training 32 (2): 136 - 140. 1997.

94 Miller PJ (1985) Assessment of joint motion. In: Rothstein, J M (Ed.) Measurement in Physical Therapy, Churchill Livingstone, New York, pp 104-109, 1985.

95 Miller EA et al., Prophylactic ankle bracing. Pediatric Clinic North Am; 37 (5): 1175 - 1185; 1990.

96 Money SM. Biomechanical comparison of support provided by the Airstirrup Ankle Training Brace pre- and post-exercise [microfiche]. Eugene (OR): University of Oregon Microform Publications, 1994.

97 Montag HJ, Rasmussen PD, Funktionelle Verbände am Bewegungsapparat - Taping Seminar, 2. überarbeitete und ergänzte Auflage, Beiersdorf medical Bibliothek, 1993. 
98 Moor KL, Dalley AF, Clinically oriented Anatomy, fourth edition, Lippincott Williams \& Wilkins, 1999, S. 640.

99 Murray, H. and Husk, L. (2001) Effect of KinesioTM taping on proprioception in the ankle. Journal of Orthopedic Sports Physical Therapy 31, A-37.

100 Myburgh KH et al., The effects of ankle guards and taping on joint motion before, during, and after a squash match. American Journal of Sportsmedicine; 12 (6): 441 - 446; 1984.

101 Nawoczenski DM et al., Objective evaluation of peroneal response to sudden inversion stress. Journal of Orthop. Sports Physical Therapy. 7: 107-109, 1985.

102 Netter FH. Atlas of Human Anatomy, Second Edition, 1997.

103 Niethard FU et al., Orthopädie - Duale Reihe, 2. Auflage, Hippokrates Verlag, 1992.

104 Nitz AJ, et al. Nerve injury and grade II and III ankle sprains. American Journal of Sports Medicine 13: 177 - 182. 1985.

105 Norris CM. Taping: components, application and mechanisms. Sports Exercise Injury; 1 (1): 14 - 17; 1994.

106 Noyes FR, Functional Properties of knee ligaments and alterations induced by immobilizations. Clinical Orthopedics 123: 210 - 224, 1977. 
107 Paris DL, The effects of the Swede-O, New Cross, and McDavid ankle braces and adhesive ankle taping on speed, balance, agility, and vertical jump. Journal of Athletic Training;27 (3): 253 - 256; 1992.

108 Paris DL. Comparisons of tape and commercial braces on ankle support, performance, injury prevention, and budget. In: Tenenbaum G, Raz Liebermann T, editors. $2^{\text {nd }}$ Maccabiah Wingate International Congress on Sport and Coaching Sciences. Netanya (Israel): Wingate Institute, 255 - 258; 1993.

109 Paris DL et al., Ankle ranges of motion during extended activity periods while taped and braced. Journal of Athletic Training; 30 (3): 223 - 228; 1995.

110 Passarello AJ et al., Improving traditional ankle taping techniques with rigid strapping tape. Journal of Athletic Training 29 (1): 76 - 77; 1994.

111 Perlman $M$ et al., Inversion lateral ankle trauma: differential diagnosis, review of literature, and prospective studies. Journal of Foot Surgery; 26: 95-135; 1987.

112 Pienkowski D et al., The effect of ankle stabilizers on athletic performance. A randomized prospective study. American Journal of Sports Med; 23 (6): 757 - 762; 1995.

113 Pinkowski IL et al., Prophylactic knee and ankle orthoses. In: Renstroem PAFH, editor. Sports Injuries: basic principles of prevention. Boston; Blackwell Scientific Publications, 1993; 374 - 387. 
114 Rarick GL, The measurable support of the ankle joiint by conventional methods of taping. Journal of Bone and Joint Surgery Am; 44 (6): 1183 - 1190; 1962.

115 Reisberg S et al., Reusable prophylactic ankle support: A review of the literature. Journal of Sport Rehabilitation; 1 (4): 290 - 299; 1992.

116 Reisberg S et al., Reusable prophylactic ankle support: A review of the literature. Journal of Sport Rehabilitation; 2 (1): 43 - 52; 1993.

117 Richard MD, et al., Effects of tape and exercise on dynamic ankle inversion. Journal of Athletic Training 35: 31 - 37. 2000.

118 Riemann, B. and Lephart, S. (2002) The sensorimotor system, Part II: The role of proprioception in motor control and functional joint stability. Journal of Athletic Training 37, 80-84.

119 Ritzdorf, W./ Condrad, A. A Biomechanical Analysis of the Highjump. In: International Athletic Foundation: Scientific Report of the II. World Championships in Athletics, Rom 1987, Book 2

120 Robbinson JR et al., Systematic ankle stabilisation and the effect on performance, Med Sci Sports Exerc, 18 (6): 625 - 628; 1986.

121 Robbins S, et al. Factors associated with ankle injuries - preventive measures. Sports Medicine 25:63 - 72. 1998.

122 Robbins S., et al. Ankle taping improves proprioception before and after exercise in young men. British Journal of Sports Medicine 29, 242247; 1995. 
123 Robichaud JA, et al., Air-splint pressure on soleus muscle alpha motorneuron reflex excitability on subjects with spinal cord injury. Archives of Physical Medicine and Rehabilitation 77: 778 - 782. 1996.

124 Rovere GD et al., Retrospective comparison of taping and ankle stabilizers in prevention of ankle injuries. Am Journal of Sports Medicine, 16 (3): 228 - 233; 1988.

125 Ryan AJ et al., Ankle sprains: A round tble. Physician Sports Med; 14 (2): 101-108; 1986.

126 Ryan L, Mechanical stability, muscle strength and proprioception in functionally unstable ankle. Australian Journal of Physiotherapy 40: 41 47. 1994.

127 Scheuffelen CA et al., Neuartige funktionelle Untersuchungen zum Stabilisierungsverhalten von Sprunggelenksorthesen. Sportverletzung \& Sportschaden. 7: 30-36, 1993.

128 Scott JZ. Effect of the active ankle brace in controlling ankle plantarflexion, inversion stress before and after exercise. Eugen (OR): University of Oregon, 1994.

129 Sharpe SR et al., Ankle braces effectively reduce recurrence of ankle sprains in female soccer players. Journal of Athletic Training, 32 (1): 259 - 262; 1997.

130 Shawdon A et al., Injury profile of amateur Australian rules footballers. Australian J Sci Med Sport 1994; 26 (3-4): 59-61. 1994. 
131 Simoneau, G., Degner, R., Kramper, C. and Kittleson, K. (1997) Changes in ankle joint proprioception resulting from strips of athletic tape applied over the skin. Journal of Athletic Training 32, 141-147.

132 Sitler $M$ et al., The efficacy of a semirigid ankle stabilizer to reduce acute ankle injuries in basketball. A randomized clinical study at West Point. American Journal of Sports Medicine, 22 (4):454 - 461; 1994.

133 Sitler MR, Role of prophylactic knee and ankle bracing in injury reduction. Journal of Sports Rehabilitation. 1 (3): 223 - 236; 1992.

134 Sjostrom M, Fugl-Meyer, AR \& Wahlby, L (1978) Achilles tendon injury: plantarflexion strength and structure of the soleus muscle after surgical repair. Acta Chirurgica Scandinavica, 144, 219-226; 1978.

135 Soutas-Little RW et al. (1987) Analysis of foot motion during running using a joint coordinate system. Medicine and Science in Sports and Exercise, 19, 285-293; 1978.

136 Springings EJ, et al., An EMG analysis of the effectiveness of external ankle support during sudden ankle inversion. Canadian Journal of Sport Sciences; 6(2): 72 - 75; 1981.

137 Stiell I et al. Implementation of the Ottawa ankle rules. Journal of the American Medical Association, 271: 827 - 832, 1994.

138 St Pierre T et al., The tensile strength of the anterior talofibular ligament. Foot Ankle; 4: 83 - 85; 1983. 
139 Stuessi E et al., A biomechanical study of the stabilization effect of the Aircast Ankle Brace. In: Jonsson B, editor. Biomechanics XA. Champaign IL: Human Kinetics Publishers 1987, 159 - 164; 1987.

140 Subotnick SI, Foot and ankle biomechanics in walking and running. In : Subotnick SI, editor. Sports medicine of the lower extremity. New York: Churchill Livingstone, 159- 178; 1989.

141 Swaddling, J. (1984). The ancient Olympic Games. Austin: University of Texas Press

142 Tropp H, Functional instability of the ankle joint. Medical dissertation no. 202, 1 - 92, Linköping University, Sweden, Linköping VTT Grafiska, 1985.

143 Tweedy R, et al. Leuko and Nessa Ankle Braces: effectiveness before and after exercise. Australian Journal of Sci Med Sport 26(3-4): 62 - 66. 1994.

144 Vaes $P$, et al. Comparative radiological study of the influence of ankle joint strapping and taping on ankle stability. Journal of Orthop Sport Phys Therapy, 7(3): 110 - 114, 1985.

145 Vaes $\mathrm{P}$, et al. Comparative radiologic study of the influence of ankle joint bandages on ankle stability. American Journal of Sportsmedicine; 13 : 157 - 171, 1998.

146 Vaes $P$, et al. Influence of Ankle Strapping, Taping, and Nine Braces: A Stress Roentgenologic Comparison. Journal of Sport Rehabilitation, 7: 157 - 171, 1998. 
147 Vaes $P$, et al. Supine versus erect ankle stress roentgen procedure in functionally unstable ankles. Conference proceedings, EFORT Barcelona, April 1997, Journal of Bone and Joint Surgery (B) suppl., September 1997.

148 Verbrugge JD, The effects of semirigid Air-Stirrup bracing vs. Adhesive ankle taping on motor performance. Journal of Orhtop Sports Phys Ther, 23 (5): 320 - 325; 1996.

149 Walters, A. (2000) Analysis of the effects of ankle taping on proprioception: a comparison before and after exercise. Masters thesis, Boise State University Library.

150 Wentz, S. GOTS-Manual Sporttraumatologie. Verlag Hans Huber, 1997, $243-247$

151 Wiley JP, et al. The effect of an ankle orthosis on ankle range of motion and performance. Journal of Orthop Sports Phys Therapy 13: 362 - 369. 1996.

152 Wiley JP, et all., The effect of an ankle orthosis on ankle range of motion and performance. Journal of Orthop Sport Phys Ther, 23 (6): 362 - 369; 1996.

153 Wilkerson GB. Comparative biomechanical effects of the standard method of ankle taping and a taping method designed to enhance subtalar stability. American Journal of Sportsmedicine, 19 (6): 588 - 595; 1991. 
154 Wischmann, B. Der Weg zum Erfolg in der Leichtathletik. Wiesbaden 1987.

155 Zacharias Th. Hochsprung lehren - Mittel und Wege. Mainz 1978. 


\section{DANKSAGUNG}

Mein besonderer Dank gilt dem Betreuer meiner Arbeit, Herrn Prof. Dr. Arnd Krüger,

der mir nicht nur die Möglichkeit gab eine trainings- und sozialwissenschaftlich wichtige Studie als Dissertation einzureichen sondern mir auch stets mit inhaltlich wertvollen Anregungen zur Fertigstellung meines Manuskriptes geholfen hat und als Erstgutachter zur Verfügung stand.

Herrn Prof. Dr. Andree Niklas danke ich für die Abnahme des Zweitgutachtens.

All den Menschen zu danken, die mir als Probanden ihre wertvolle Zeit schenkten, würde den Rahmen dieser Danksagung sprengen.

Herrn Friedrich Kehrer von der Werbeagentur EDITION - K in Regensburg danke ich für die Gestaltung des Deckblattmotives.

Weit mehr als ein großes 'Dankeschön' gebührt meiner Frau, Astrid und meinen zwei Töchtern, die meinen Einsatz zur Fertigstellung dieser Arbeit nicht nur kompensierend geduldet, sondern motivierend unterstützt haben. Ihnen ist diese Arbeit gewidmet.

Regensburg, 28. Oktober 2004 


\section{curriculum vitae \\ HOMAYUN GHARAVI - NOURI}

Geboren am 30. Juli 1968 in Teheran/Iran, als Sohn des Iranischen Diplomaten Dr. Ali GharaviNouri und seiner Ehefrau Asadeh, geb. Shahidi.

1988

$1988-1996$

$1989-1995$

$1997-2000$

$06 / 2000$

$2000-2001$

$07 / 2001-11 / 2003$

2002

2004
Hochschulreife, Otto-Kühne-Gymnasium, Bonn

Studium und Staatsexamen Humanmedizin, Uni Göttingen

Studium der Sportwissenschaften, Uni Göttingen

Stipendium am 'Exercise Science Department' der University of California, Davis, USA

Erlangung des U.S. ECFMG Zertifikats

'Associate Professor' für Anatomie und Pathologie, ClevelandCollege Los Angeles, California, USA

Assistenzarzt Uniklinik Regensburg, Orthopädie und Chirurgie

Erlangung des Doktorgrades der Medizin, Universität Regensburg Erlangung der Zusatzbezeichnung 'Sportmedizin', BLÄK

Autor und Herausgeber von 'Ambulanz live'

Problemorientiertes Lernen

Band 1 und 2, Verlag Elsevier, München.

Produzent, Autor und Herausgeber von ATHLETIC TAPING I + II, Verlag SPORTSMED SOLUTIONS, Regensburg. 